Aus dem

Institut für klinische Neurophysiologie

(Chefärztin: Prof. Dr. med. S. Happe)

des Klinikums Bremen-Ost in Bremen

\title{
Randomisierte, kontrollierte Parallelgruppenstudie zur Untersuchung der Wirksamkeit der manuellen Lymphdrainage und klassischen Massage zur Prophylaxe der Migräne mit und ohne Aura
}

\author{
Inaugural-Dissertation \\ zur Erlangung des Doktorgrades \\ der Medizinischen Fakultät \\ der Georg-August-Universität zu Göttingen
}

\author{
vorgelegt von \\ Minhal Hamzeh \\ aus \\ Stef/Algerien
}

Göttingen 2012 
Dekan: Prof. Dr. med. C. Frömmel

I. Berichterstatterin: Prof. Dr. med. S. Happe

II. Berichterstatter: Prof. Dr.rer. bio.hum. Pfingsten

III. Berichterstatter: Priv.-Doz. Dr. med. Klinger

IV. Berichterstatter: Prof. Dr. med. Oppermann

Tag der mündlichen Prüfung: 03.01.2012 


\section{Inhaltsverzeichnis}

1.

Einleitung....... 1

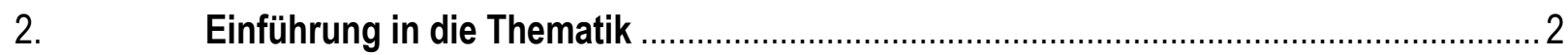

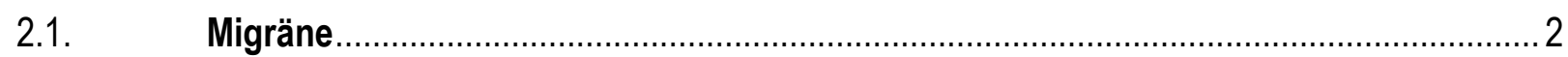

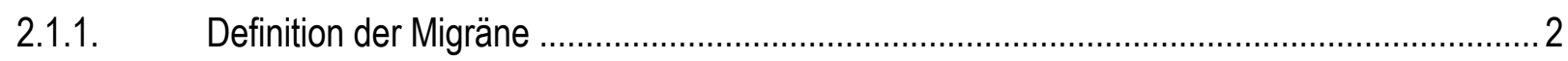

2.1.2. Diagnose und Differenzialdiagnose ........................................................................ 2

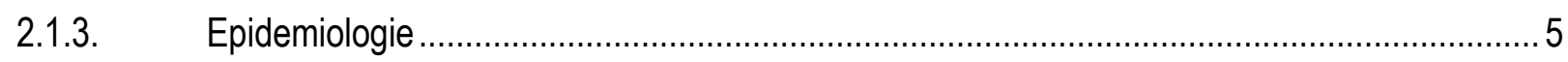

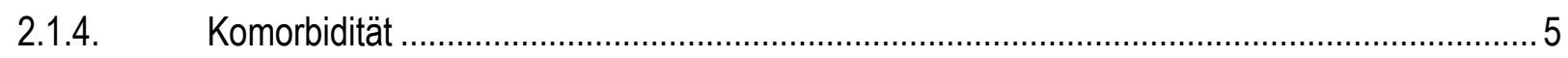

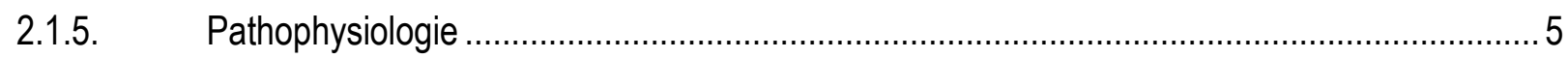

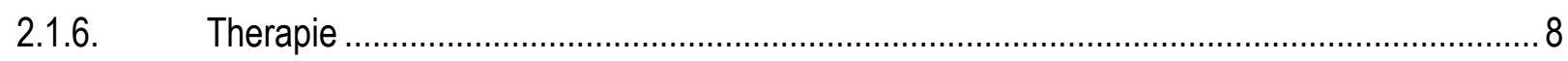

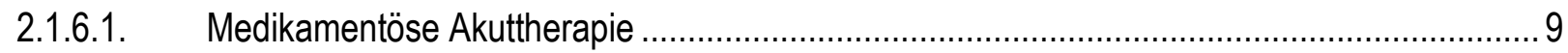

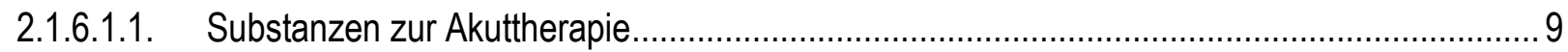

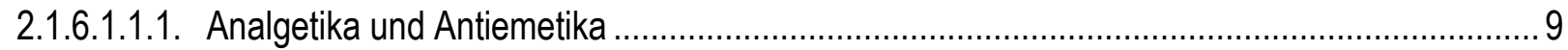

2.1.6.1.1.2. 5-HT1B/1D-Agonisten (Triptane) ……........................................................................ 11

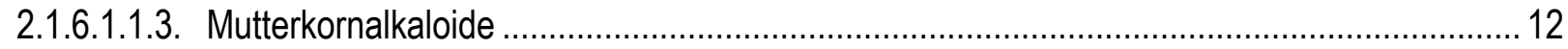

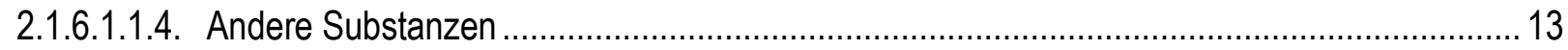

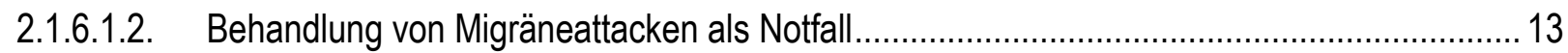

2.1.6.2. Medikamentöse Migräneprophylaxe ........................................................................... 13

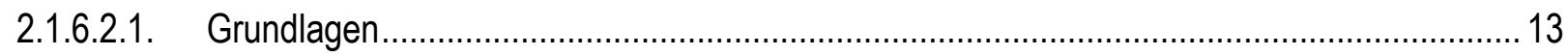

2.1.6.2.2. Substanzen zur Migräneprophylaxe .................................................................... 14

2.1.6.2.2.1. Substanzen der ersten Wahl ................................................................................. 14

2.1.6.2.2.2. Substanzen der zweiten Wahl .............................................................................. 16

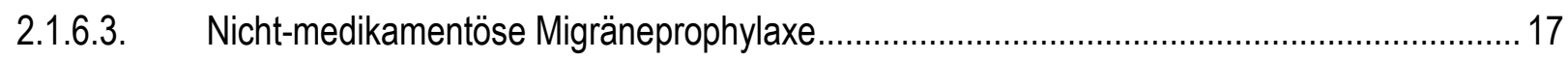

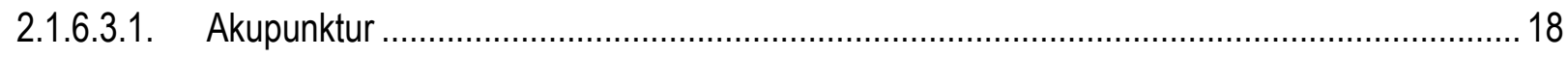

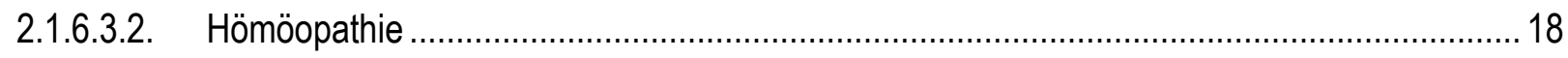

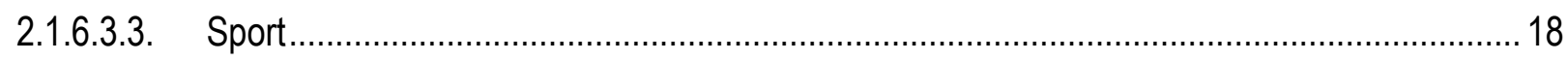


2.1.6.3.4. Verhaltenstherapie der Migräne …........................................................................ 18

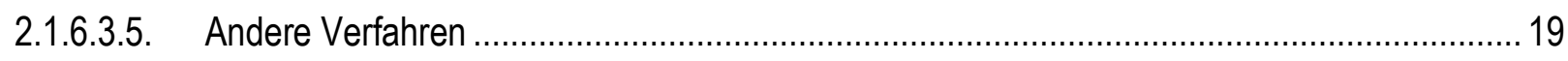

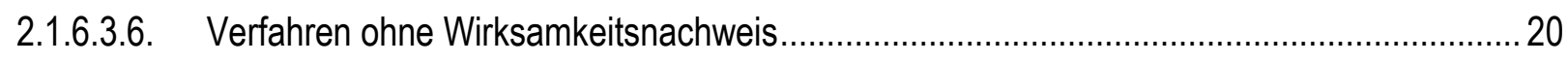

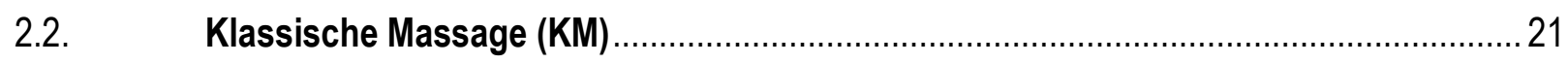

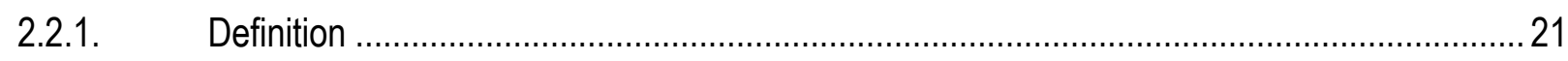

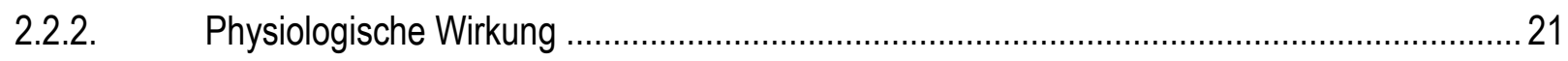

2.2.2.1. Mechanische Wirkkomponenten.......................................................................... 22

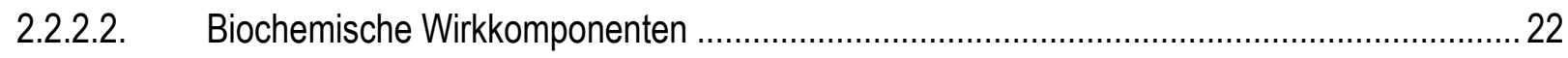

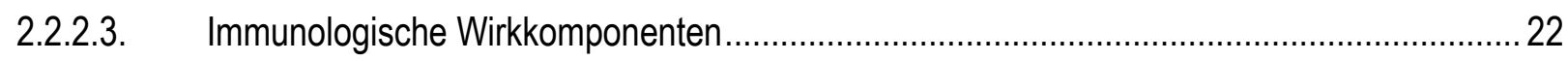

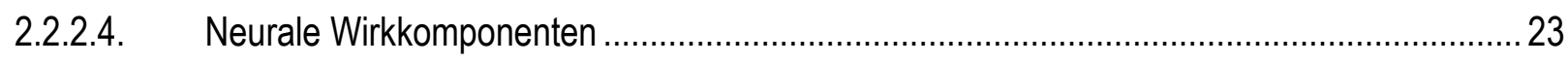

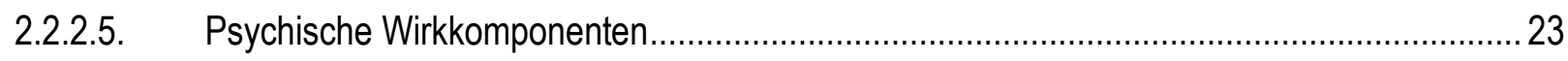

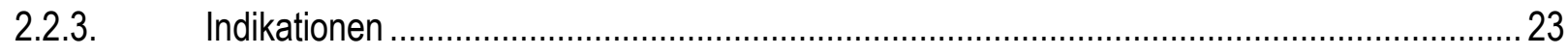

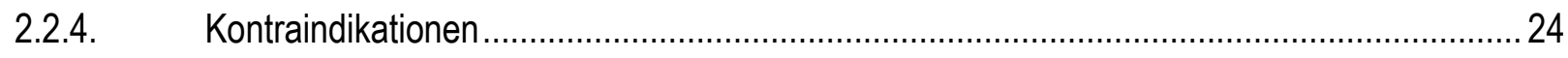

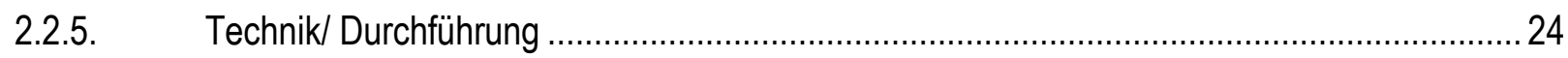

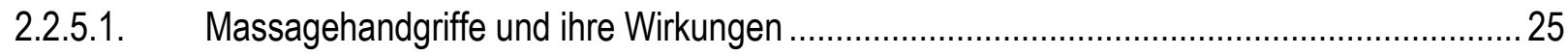

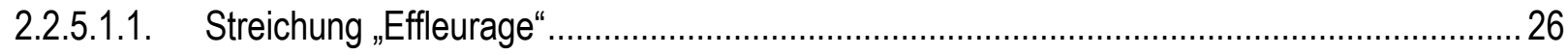

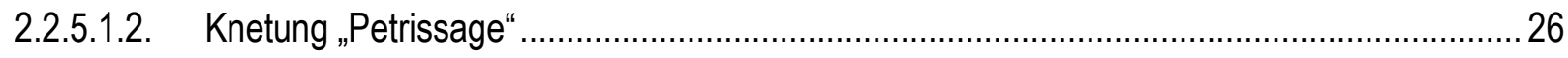

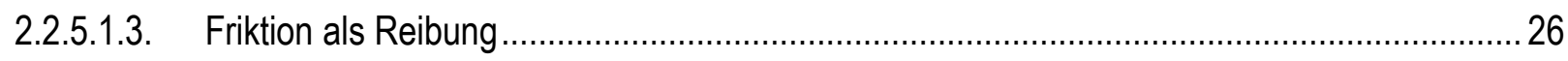

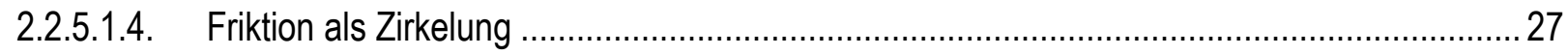

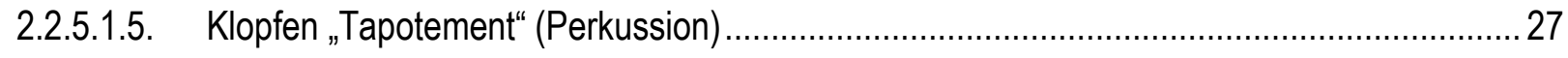

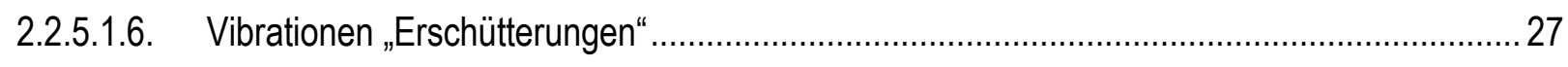

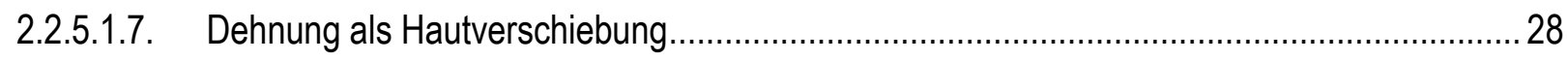

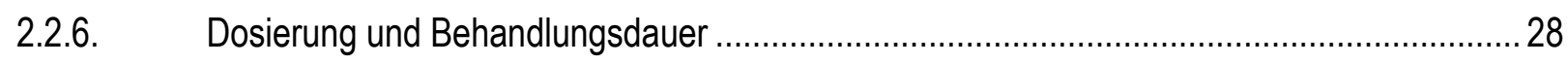

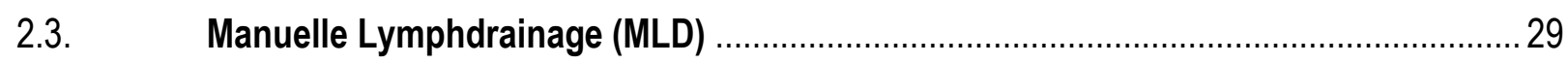

2.3.1. Historische Entwicklung der manuellen Lymphdrainage ............................................... 29

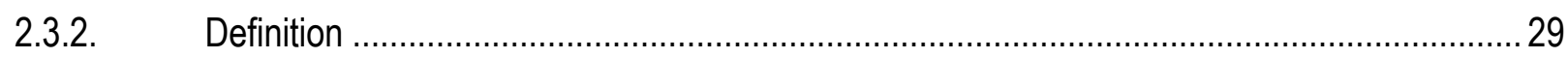




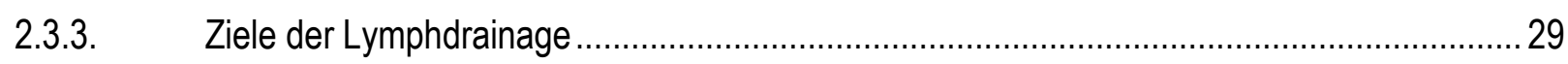

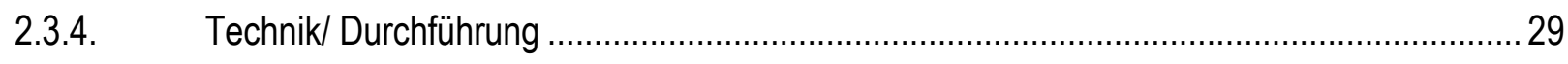

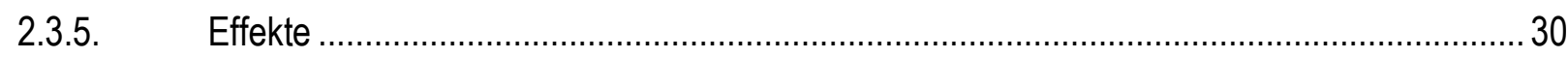

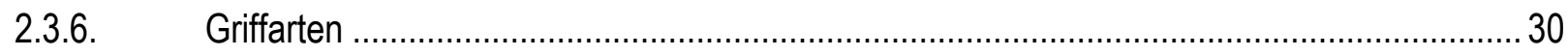

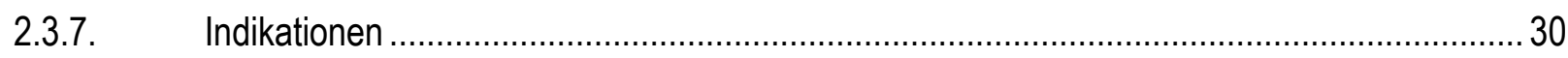

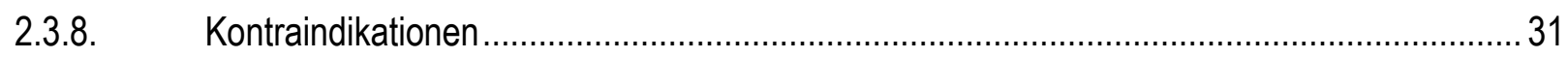

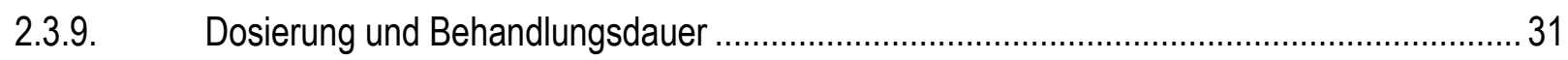

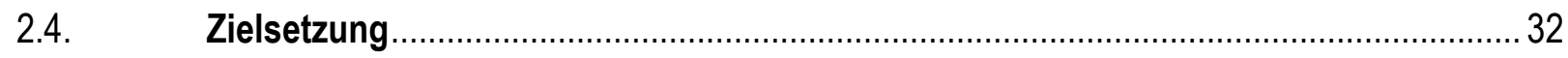

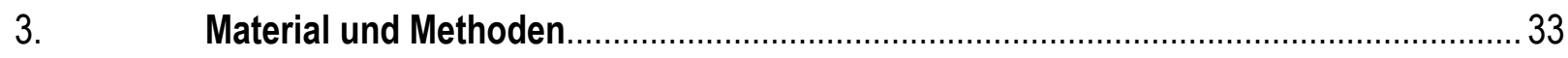

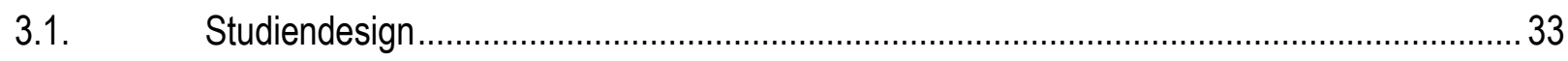

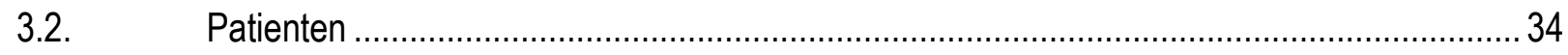

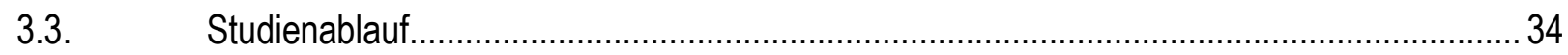

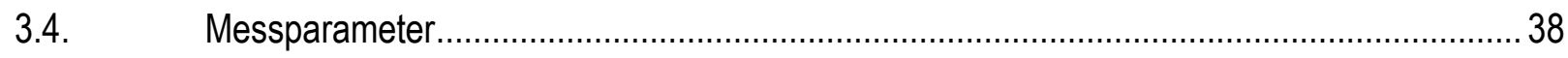

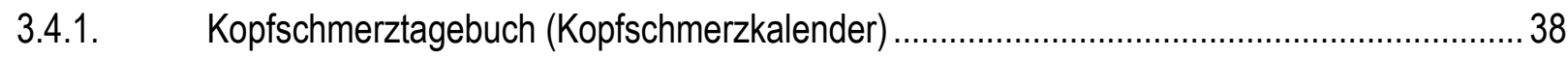

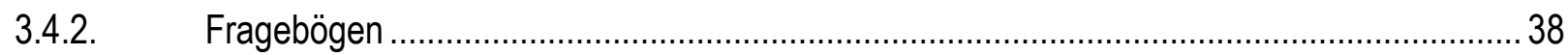

3.4.2.1. Inventar zur Beeinträchtigung durch Kopfschmerzen ................................................... 39

3.4.2.2. Migräne disability assessment, Midas-Fragebogen ....................................................... 39

3.4.2.3. Center for Epidemiologic Studies Depression Scale, CES-D ........................................... 40

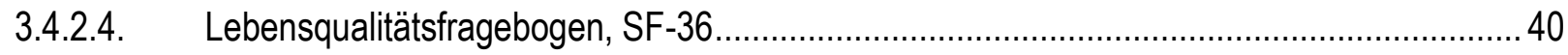

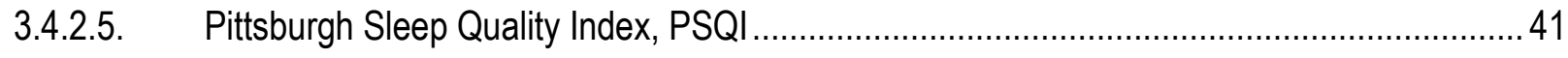

3.4.2.6. Clinical Global Impression Scale, CGI ............................................................... 41

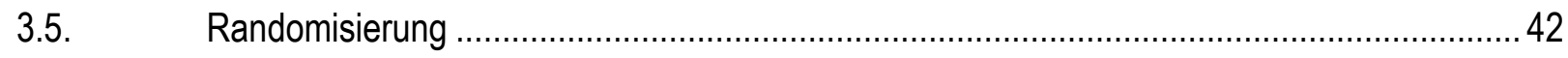

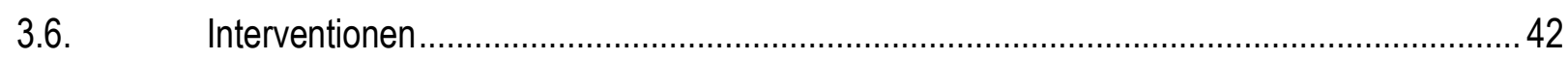

3.6.1. Behandlungsprotokoll für Gruppe 1 und Gruppe 3a „Manuelle Lymphdrainage Gesicht“ ..... 43

3.6.2. Behandlungsprotokoll für Gruppe 2 und Gruppe 3b „Klassische Rückenmassage“ ............. 45

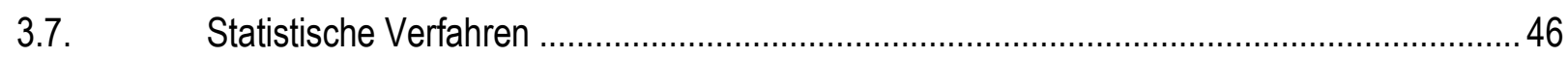


4. Ergebnisse

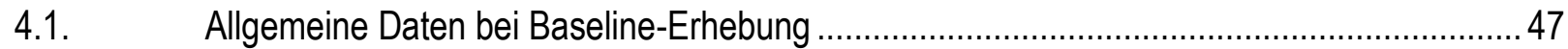

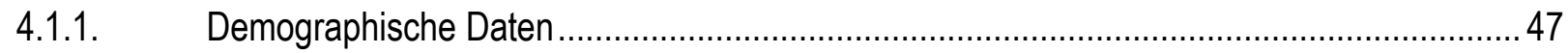

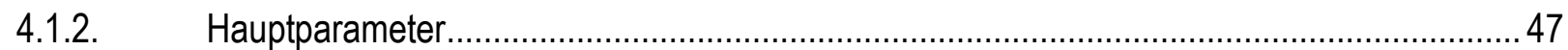

4.1.3. Begleitsymptome, Schmerzintensität und psychometrische Parameter ............................ 48

4.2. Veränderung der Parameter im Verlauf der Untersuchung in der LD-Gruppe ..................... 49

4.2.1. Veränderung der Hauptparameter ................................................................... 49

4.2.2. Veränderung der Begleitsymptome und der Schmerzintensität ........................................50

4.2.3. Veränderung der psychometrischen Parameter .......................................................... 51

4.3. Veränderung der Parameter im Verlauf der Untersuchung in der KM-Gruppe ....................53

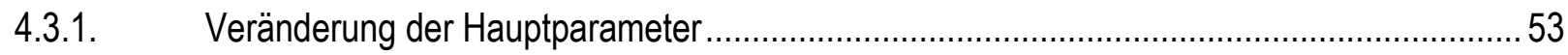

4.3.2. Veränderung der Begleitsymptome und der Schmerzintensität .........................................54

4.3.3. Veränderung der psychometrischen Parameter ............................................................... 55

4.4. Veränderung der Parameter im Verlauf der Untersuchung in der Wartegruppe (WG).........57

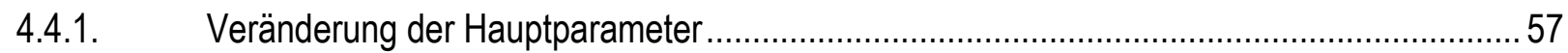

4.4.2. Veränderung der Begleitsymptome und der Schmerzintensität ..........................................58

4.4.3. Veränderung der psychometrischen Parameter .............................................................5

4.5. Responderrate und Hauptparameter aller Gruppen in Vergleich ......................................62 62

4.5.1. Zwischen Baseline und Nachbeobachtung (Primäre Endpunkte) .....................................62 62

4.5.2. Zwischen Baseline und Behandlungsende (nach 8 Wochen; sekundäre Endpunkte) ..........66

4.6. Begleitsymptome und Schmerzintensität aller Gruppen im Vergleich................................. 68

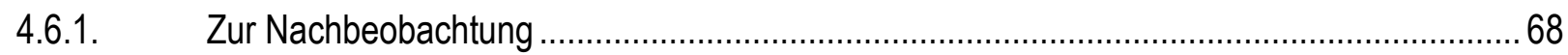

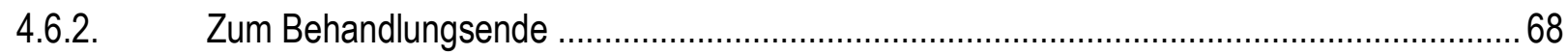

4.7. Veränderung der psychometrischen Parameter aller Gruppen im Vergleich ......................69

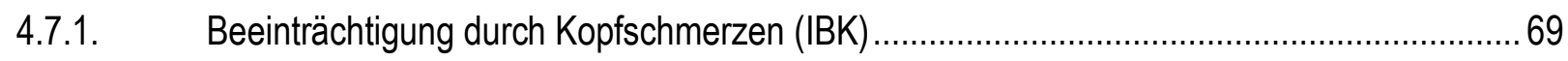

4.7.2. Einschränkung der Leistungsfähigkeit (Midas) ........................................................ 70 


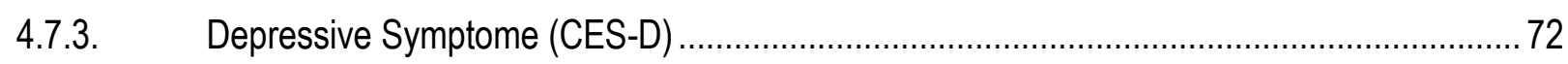

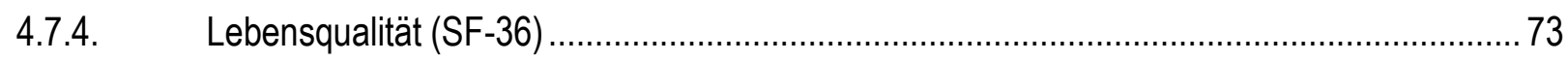

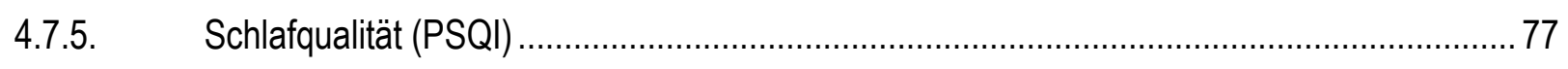

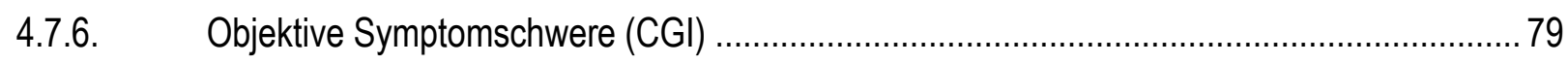

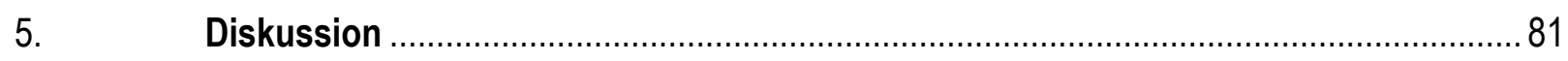

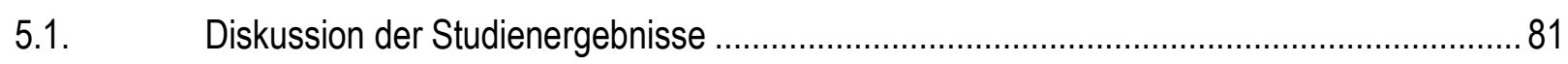

5.1.1. Wirkung der manuellen Lymphdrainage auf Kopfschmerztage, Migräneattacken, Migränetage, akute Schmerzmedikation und Schmerzintensität ..................................... 81

5.1.2. Wirkung der klassischen Massage auf Kopfschmerztage, Migränetage, Migräneattacken, akute Schmerzmedikation und Schmerzintensität ....................................................... 82

5.1.3. Wirkung der manuellen Lymphdrainage auf Begleitsymptome....................................... 83

5.1.4. Wirkung der klassischen Massage auf Begleitsymptome ................................................ 83

5.1.5. Wirkung der manuellen Lymphdrainage und klassischen Massage auf psychometrische

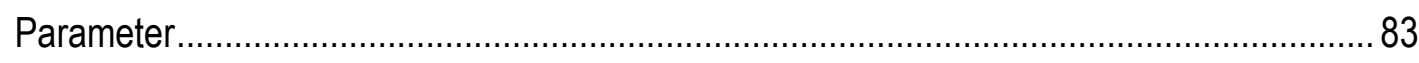

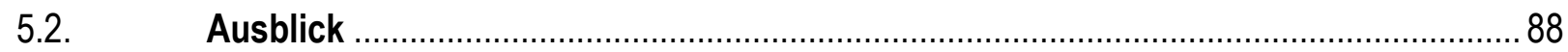

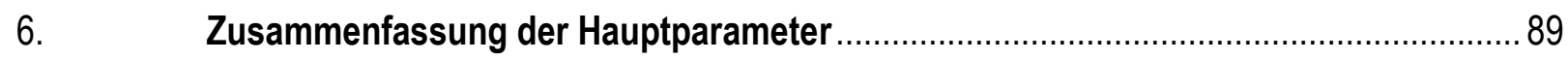

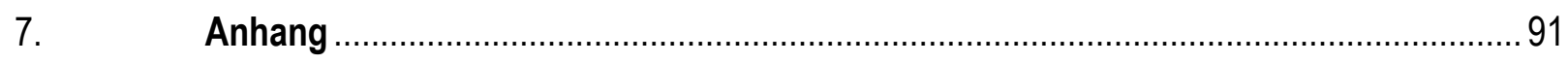

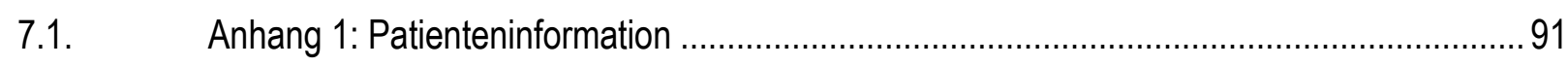

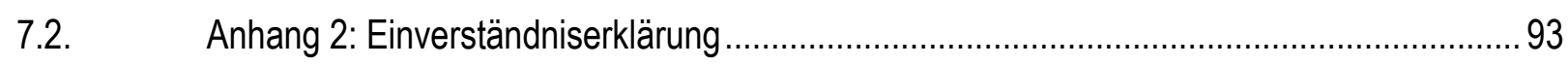

7.3. Anhang 3: Standardisierter Erhebungsbogen der demographischen und klinischen Daten .94

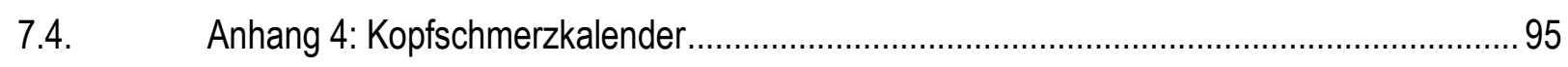

7.5. Anhang 5: Inventar zur Beeinträchtigung durch Kopfschmerzen, IBK (Bauer et al.1999).....96

7.6. Anhang 6: Migraine disability assessment, Midas Fragebogen (Stewart et al. 1999) ..........97

7.7. Anhang 7: Center for Epidemiologic Studies Depression Scale, CES-D (Andresen et al.

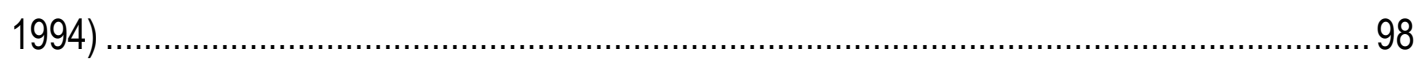

7.8. $\quad$ Anhang 8: Lebensqualitätsfragebogen, SF-36 (Ware et al. 1993) ..................................... 99

7.9. $\quad$ Anhang 9: Pittsburgh Sleep Quality Index, PSQI (Buysse et al. 1989) ............................ 103 
7.10. Anhang 10: Clinical Global Impression Scale, CGI (Guy 1976) ..................................... 107

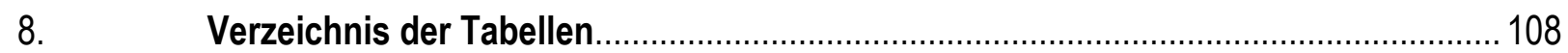

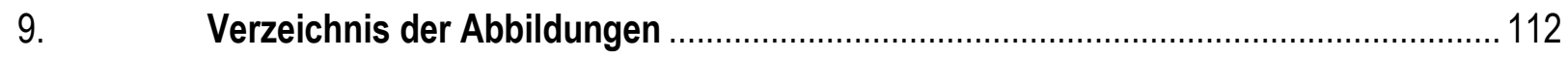

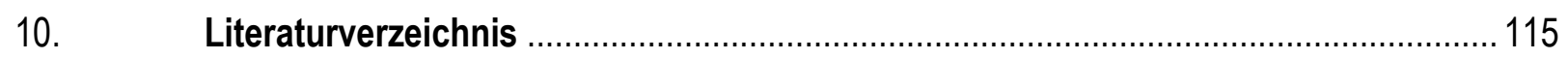

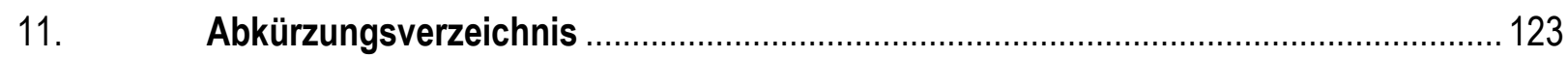




\section{Einleitung}

Kopfschmerzen stellen eines der häufigsten Symptome in der allgemeinärztlichen Praxis dar. Über $90 \%$ aller Menschen haben wenigstens einmal in ihrem Leben behandlungsbedürftige Kopfschmerzen. Die International Headache Society (IHS) entwickelte für Kopfschmerzen eine exakte Klassifikation, die 1988 erstmals publiziert worden und 2004 in revidierter Fassung neu erschienen ist (Headache Classification Subcommittee of the International Headache Society 2004). Hierbei werden Kopfschmerzen in zwei große Hauptgruppen unterteilt: idiopathische (=primäre) und symptomatische (=sekundäre) Kopfschmerzen. Über 95\% aller Kopfschmerzen gehören zur Gruppe der idiopathischen, von denen die Migräne und der Kopfschmerz vom Spannungstyp die wichtigsten sind. Durch eine exakte Klassifikation der Kopfschmerzen ist auch eine moderne evidenzbasierte Therapie möglich, die in Form von Empfehlungen regelmäßig von der Deutschen Migräne- und Kopfschmerzgesellschaft (DMKG) publiziert werden. Für die Akutbehandlung und Prophylaxe der Migräne stehen heute zahlreiche wirksame Medikamente zur Verfügung (Diener et al. 2005). Als nicht-medikamentöse Verfahren sind evidenzbasiert wirksam: Aerober Ausdauersport, Entspannungsverfahren, verschiedene verhaltenstherapeutische Verfahren und fraglich auch Akupunktur. Methoden der physikalischen Therapie zur Prophylaxe der Migräne sind, obwohl häufig angewendet und empirisch wirksam, bislang nur unzureichend untersucht. Insbesondere für die klassische Massage und weniger für Lymphdrainage gibt es Hinweise, dass sie in der Prophylaxe der Migräne wirksam sein können (Krahl 2005, Lawler und Cameron 2006).

Im Rahmen dieser Arbeit soll darum bei Patienten mit Migräne mit und ohne Aura, die eine standardisierte Behandlung mit Lymphdrainage oder klassischer Massage zur Migräneprophylaxe erhalten, untersucht werden:

a) ob die Anzahl der Migränetage und der Migräneattacken unter der Behandlung mit Lymphdrainage oder klassischer Massage abnimmt (definiert als Abnahme der Attacken pro Monat um mindestens $50 \%$ gegenüber einem Baseline-Monat, sogen. Responder),

b) ob die Schmerzintensität und die Anzahl der eingenommenen Akutmedikamente abnehmen,

c) ob depressive Symptome abnehmen,

d) ob die Lebensqualität zunimmt und

e) ob sich die Schlafqualität verbessert. 


\section{Einführung in die Thematik}

\subsection{Migräne}

\subsubsection{Definition der Migräne}

Die International-Headache-Society (IHS)-Klassifikation in der überarbeiteten Version von 2004 unterteilt die Migräne in zwei Haupttypen: 1.1 Migräne ohne Aura und 1.2 Migräne mit Aura.

Migräne ohne Aura wird wie folgt definiert: „Primäre wiederkehrende Kopfschmerzerkrankung, die sich in Attacken von 4 -72 Stunden Dauer manifestiert. Typische Kopfschmerzcharakteristika sind einseitige Lokalisation, pulsierender Charakter, mäßige bis starke Intensität, Verstärkung durch körperliche Routineaktivitäten und begleitet von Übelkeit und/oder Licht- und Lärmüberempfindlichkeit“" (Evers und Göbel 2004).

Migräne mit Aura wird wie folgt definiert: „Wiederkehrende Erkrankung mit anfallsweise auftretenden reversiblen fokalen neurologischen Symptomen, die sich allmählich über 5-20 Minuten hinweg entwickeln und weniger als 60 Minuten anhalten. In der Regel folgen diesen Aurasymptomen Kopfschmerzen, die die Charakteristika einer Migräne ohne Aura aufweisen“ (Evers und Göbel 2004).

\subsubsection{Diagnose und Differenzialdiagnose}

Um die Diagnose 1.1 Migräne ohne Aura stellen zu können, müssen nach der IHS-Klassifikation folgende Kriterien erfüllt sein:

a) Mindestens 5 Attacken, welche die Kriterien b-d erfüllen.

b) Kopfschmerzattacken, die (unbehandelt oder erfolglos behandelt) 4 bis 72 Stunden anhalten.

c) Der Kopfschmerz weist wenigstens 2 der folgenden Charakteristika auf:

1. Einseitige Lokalisation

2. Pulsierender Charakter

3. Mittlere oder starke Schmerzintensität

4. Verstärkung durch körperliche Routineaktivitäten (z.B. Gehen oder Treppensteigen) oder diese führen zu deren Vermeidung

d) Während des Kopfschmerzes besteht mindestens eines:

1. Übelkeit und/oder Erbrechen

2. Photophobie und/oder Phonophobie

e) Nicht auf eine andere Erkrankung zurückzuführen.

Nach diesen Kriterien der IHS müssen die Migränekopfschmerzen nicht zwingend halbseitig oder pulsierend sein. Wichtiger als ein einzelnes Symptom (wie Halbseitigkeit der Kopfschmerzen) ist die Kombination der Schmerzen mit vegetativen Symptomen. 
Die Differenzialdiagnose umfasst alle anderen wiederkehrenden Kopfschmerzsyndrome wie Spannungskopfschmerz und Cluster-Kopfschmerz. Es ist nicht immer einfach, diese Differenzialdiagnosen auszuschließen. Ausführliche Anamnese sowie allgemeinmedizinische und eine neurologische körperliche Untersuchung spielen die wichtigste Rolle; wichtige Faktoren, die für die Diagnose einer Migräne sprechen, sind eine Migränehäufigkeit in der Familiengeschichte, die Relation zwischen hormoneller Veränderung (Menstruation, Ovulation, Schwangerschaft, Stillzeit) und Kopfschmerzen, Stress sowie Zunahme der Kopfschmerzen unter körperlicher Belastung wie Treppensteigen. Nur bei dem Verdacht auf das Vorliegen von sekundären Kopfschmerzen ist eine weitere Zusatzdiagnostik wie die zerebrale Bildgebung notwendig. Eine spezifische Migränediagnostik gibt es nicht. Als unspezifischer Befund bei Migränepatienten sind unspezifische Allgemeinveränderungen (paroxysmale, generalisierte Dysrhythmien) im EEG und unspezifische Flussbeschleunigung in der Dopplersonographie (May 2006). Problematisch in der Differenzialdiagnose kann das gemeinsame Auftreten der Migräne mit einem episodischen Kopfschmerz vom Spannungstyp oder mit einem zervikogenen Kopfschmerz sein. Hier kann der Kopfschmerzkalender helfen, verschiedene Kopfschmerzarten besser zu unterscheiden. Diese Unterscheidung ist wichtig, um einem unselektiven Gebrauch von Schmerzmitteln vorzubeugen. Schließlich kann es auf Grundlage einer Migräne durch die missbräuchliche Einnahme von Migränemitteln (Analgetika, ergotaminhaltige Präparate, Triptane) zu einem Dauerkopfschmerz kommen, der nur durch eine Entzugsbehandlung durchbrochen werden kann. Hierbei handelt es sich um einen täglich oder fast täglich auftretenden Kopfschmerz, der intermittierend in eine typische Migräne übergehen kann und durch die (fast) tägliche Einnahme von Analgetika oder andere akute Migränemedikamente unterhalten wird. Dieses Phänomen ist auch bei anderen idiopathischen Kopfschmerzerkrankungen zu beobachten, jedoch nicht bei anderen Schmerzerkrankungen. Die definitive Diagnose eines medikamentös induzierten Dauerkopfschmerzes kann erst gestellt werden, wenn nach Absetzen der Medikamente der Kopfschmerz auch wieder abklingt. 


\begin{tabular}{|c|c|c|c|}
\hline Differenzialdiagnose & $\begin{array}{l}\text { Kopfschmerz und typische } \\
\text { Anamnese }\end{array}$ & $\begin{array}{l}\text { Typisches Alter und } \\
\text { Patientenpopulation }\end{array}$ & $\begin{array}{l}\text { Notwendige weitere } \\
\text { Untersuchungen }\end{array}$ \\
\hline Clusterkopfschmerz & $\begin{array}{l}\text { Kopfschmerzdauer kürzer und } \\
\text { intensiver als bei Migräne (15-45 } \\
\text { min), Rhinorrhö, Ptosis, } \\
\text { Lakrimation, keine Übelkeit oder } \\
\text { Erbrechen, bis zu } 3 \text { Attacken pro } \\
\text { Tag }\end{array}$ & $\begin{array}{l}\text { Männer jüngeren und } \\
\text { mittleren Alters }\end{array}$ & $\begin{array}{l}\text { CCT/NMR initial zum } \\
\text { Ausschluss anderer } \\
\text { Ursachen }\end{array}$ \\
\hline Trigeminus-Neuralgie & $\begin{array}{l}\text { Schmerzen wesentlich kürzer als } \\
\text { bei Migräne oder } \\
\text { Clusterkopfschmerz (nur } \\
\text { Sekunden!), dutzende Attacken } \\
\text { pro Tag }\end{array}$ & $\begin{array}{l}\text { Ältere Patienten } \\
\text { (über } 60 \text { Jahre) }\end{array}$ & $\begin{array}{l}\text { CCT/NMR initial zum } \\
\text { Ausschluss anderer } \\
\text { Ursachen }\end{array}$ \\
\hline $\begin{array}{l}\text { Subarachnoidalblutung } \\
\text { (SAB) }\end{array}$ & $\begin{array}{l}\text { Heftigster nie gekannter, plötzlich } \\
\text { aufgetretener Kopfschmerz, } \\
\text { aufgetreten nach Anstrengung, } \\
\text { Bewegungseinschränkung, } \\
\text { Meningismus }\end{array}$ & Jedes Alter & $\begin{array}{l}\text { CCT, } \\
\text { Liquoruntersuchung: } \\
\text { Blutiger Liquor. } \\
\text { Dann Angiographie: } \\
\text { Aneurysma? }\end{array}$ \\
\hline $\begin{array}{l}\text { Intrazerebrale Blutung } \\
\text { (ICB) }\end{array}$ & $\begin{array}{l}\text { Heftige Kopfschmerzen, fokale } \\
\text { neurologische Symptome, } \\
\text { epileptische Anfälle, vegetative } \\
\text { Unruhe }\end{array}$ & Jedes Alter & $\begin{array}{l}\text { CCT } \\
\text { Blutungsneigung } \\
\text { Marcumar?, } \\
\text { Hochdruckkrise? }\end{array}$ \\
\hline Arteriitis temporalis & $\begin{array}{l}\text { Über Tage bis wenige Wochen } \\
\text { sich entwickelnder, heftiger, } \\
\text { einseitiger, ganztägiger } \\
\text { Kopfschmerz, u.U. } \\
\text { Visusminderung, Schmerzen } \\
\text { beim Kauen (!) }\end{array}$ & $\begin{array}{l}\text { Über } 60 \text { Jahre, nicht } \\
\text { selten mit Polymyalgia } \\
\text { rheumathica } \\
\text { gemeinsam }\end{array}$ & $\begin{array}{l}\text { BSG massiv erhöht, } \\
\text { Leukozytose, ggf. } \\
\text { Biopsie, sonst } \\
\text { klinische Diagnose }\end{array}$ \\
\hline Sinusvenenthrombose & $\begin{array}{l}\text { Langsam über Wochen (bis zu } 8 \\
\text { Wochen) zunehmende } \\
\text { Kopfschmerzen, therapierefraktär, } \\
\text { epileptische Anfälle, } \\
\text { Bewusstseinsstörungen }\end{array}$ & $\begin{array}{l}\text { Jedes Alter, häufiger } \\
\text { junge Frauen mit Pille, } \\
\text { Nikotin, } \\
\text { Schwangerschaft. }\end{array}$ & $\begin{array}{l}\text { NMR (CCT, wenn } \\
\text { Spiral-CT) }\end{array}$ \\
\hline Sinusitis & $\begin{array}{l}\text { Dumpfer Schmerz, morgens } \\
\text { Sekretabfluss }\end{array}$ & Jedes Alter & $\begin{array}{l}\text { Röntgen } \\
\text { Nasennebenhöhlen } \\
\text { CCT }\end{array}$ \\
\hline Meningitis & $\begin{array}{l}\text { Insbesondere okzipitale und } \\
\text { frontale, bilaterale } \\
\text { Kopfschmerzen, Fieber, } \\
\text { Meningismus (nicht immer stark), } \\
\text { Bewusstseinsänderung }\end{array}$ & Jedes Alter & $\begin{array}{l}\text { Liquor entzündlich } \\
\text { verändert }\end{array}$ \\
\hline Zerebrale Vaskulitis & $\begin{array}{l}\text { Diffuse, teils intensive } \\
\text { Kopfschmerzen - sehr selten (!) }\end{array}$ & $\begin{array}{l}\text { Jüngere und } \\
\text { Patienten mittleren } \\
\text { Alters }\end{array}$ & $\begin{array}{l}\text { NMR, entzündlich } \\
\text { veränderter Liquor }\end{array}$ \\
\hline Glaukomanfall & $\begin{array}{l}\text { Heftigster orbital lokalisierter } \\
\text { einseitiger Schmerz, Bulbus } \\
\text { steinhart und druckschmerzhaft }\end{array}$ & Ab 50 Jahre & $\begin{array}{l}\text { Augeninnendruck } \\
\text { messen }\end{array}$ \\
\hline Arterielle Hypertonie & $\begin{array}{l}\text { Pochender Kopfschmerz, } \\
\text { gerötetes Gesicht }\end{array}$ & Mittleres Alter & Blutdruck messen \\
\hline Arterielle Hypotonie & Macht keine Kopfschmerzen & $?$ & Keine \\
\hline
\end{tabular}




\subsubsection{Epidemiologie}

Migräne ist eine der häufigsten Kopfschmerzerkrankungen überhaupt. Sechs bis acht Prozent aller Männer und 12 bis 14 Prozent aller Frauen leiden unter einer Migräne. Bei Kindern und Jugendlichen ist Migräne seltener; nur vier bis fünf Prozent aller Kinder und Jugendlichen leiden unter einer Migräne (Winner et al. 1997). Zwischen dem 35. und 45. Lebensjahr liegt die höchste Inzidenz der Migräneattacken, und in diesem Alter sind Frauen dreimal häufiger betroffen als Männer. Eine interessante Beobachtung ist, dass Ärzte, die sich schwerpunktmäßig mit Kopfschmerzen beschäftigen, deutlich häufiger selber unter Migräne leiden als die Durchschnittsbevölkerung. So liegt die Migränehäufigkeit bei Kopfschmerzspezialisten über 60 Prozent (Evans et al. 2005).

\subsubsection{Komorbidität}

Für die allgemeinärztliche Praxis ist es von besonderer Bedeutung, dass Migräne eine hohe Komorbidität zu psychiatrischen Erkrankungen aufweist (nachgewiesen für die bipolaren Störungen, für bestimmte Formen der Zwangsneurosen und für Angststörungen). Diese Komorbidität ist unabhängig vom jeweiligen Krankheitsverlauf und gilt in beide Richtungen. Dies bedeutet, dass Patienten mit einer depressiven Störung (insbesondere einer Major Depression) ein höheres Risiko haben, eine Migräne zu entwickeln und umgekehrt (Lantéri-Mint et al. 2005). Depression und Angststörung sind also nicht nur eine Reaktion auf die Erkrankung Migräne. Wahrscheinlich spielen Störungen im Serotoninstoffwechsel eine Rolle in der gemeinsamen Ätiologie. Migräne mit Aura ist ein Risikofaktor für ischämische Schlaganfälle bei jungen Frauen (Kurth et al. 2005). Der Zusammenhang zwischen Migräne und offenem Foramen ovale (PFO) beruht wahrscheinlich auf einer gemeinsamen genetischen Disposition (Diener et al. 2006).

\subsubsection{Pathophysiologie}

Migräne ist ein komplexes Schmerzsyndrom, dessen Ursache nur schwer zu ergründen ist. An vaskuläre Veränderungen als Ursache der Migränekopfschmerzen denkt man seit der Arbeit von Olesen et al. (1990) nicht mehr, sondern an eine Begleiterscheinung neurogener Ereignisse bzw. neuronaler Fehlfunktionen (Ebersberger 2002). Das Verständnis zu Pathophysiologie und Ätiologie der Migräne konnte durch neuere genetische und molekularbiologische Untersuchungen, aber auch durch bildgebende Verfahren deutlich verbessert werden. Die folgende Zusammenfassung richtet sich nach den Erläuterungen von Ebersberger (2002) sowie nach Limmroth und Diener (2003). 
Seit mehr als 60 Jahren ist bekannt, dass ein dichtes Netzwerk von Nervenfasern für die Entstehung von Kopfschmerzen verantwortlich ist. Das Gehirn selbst ist schmerzunempfindlich, wohl aber die größeren zerebralen Gefäße, meningealen und pialen Gefäße, die großen venösen Sinus und die Dura, die von unmyelinisierten Fasern umgeben sind, die dem ophthalmischen Ast des Trigeminus-Ganglions sowie der oberen zervikalen Hinterwurzel in der hinteren Schädelgrube entspringen (Limmroth und Diener 2003). Zu diesem Netzwerk gehören drei verschiedene neuronale Systeme: das efferente sympathische und parasympathische System und das afferente sensorische System, das aufgrund seiner engen Interaktion mit dem Gefäßsystem als trigeminovaskuläres System bezeichnet wird (Ebersberger 2002). Die Auslöser für die Aktivierung der nozizeptiven Fasern bei primären Kopfschmerzen sind noch nicht geklärt. Aus tierexperimentellen Untersuchungen ergaben sich Hinweise auf periphere Entstehungsmechanismen, aber nicht im Sinne einer (reinen) Vasodilatation, sondern im Sinne einer „neurogenen Entzündung“ der Dura (Limmroth und Diener 2003). Dieses Phänomen wird auch als sterile Inflammation der Meningen bezeichnet. Durch Reizung der o.g. Nozizeptoren kommt es zur Freisetzung von Neuropeptiden (Calcitonin gene related peptide [CGRP], Substanz $P$, Neurokinin A) aus perivaskulären Nervenendigungen in Blutgefäße der Hirnhaut (Limmroth et al. 2001, Goadsby et al. 1988), dadurch werden eine Reihe von efferenten Funktionen ausgelöst, wie eine Aktivierung von Mastzellen, Thrombozytenaggregation, eine Aktivierung von Endothelzellen in duralen Blutleitern und eine Vasodilatation duraler Blutgefäße (Dimitriadou et al. 1991). All diese Veränderungen münden in einer Extravasation von Plasmaproteinen aus den venösen Blutleitern in die Hirnhaut (Markowitz et al. 1987). Diese führen zu einer Sensibilisierung des Nozizeptors und zu Entzündungsreaktionen im Gewebe (Details bei Peroutka (2005)). Diese meningealen oder pialen Vorgänge sind beim Menschen in vivo bisher jedoch nicht nachgewiesen worden. Zur Peptidfreisetzung ist durch Studien sowohl im Tierexperiment als auch bei Patienten in der Migräneattacke nachgewiesen worden, dass durch Aktivierung trigeminaler Fasern (Tierexperiment) und während der Migräneattacke die Konzentration von vasoaktiven Neuropeptiden wie Calcitonin gene related peptide (CGRP) im venösen Blut deutlich ansteigt und nach Gabe von DHE (Dihydroergotamin) oder Sumatriptan wieder abfällt (Goadsby 1993). Daraus darf geschlossen werden, dass es während der Migräneattacke zu einer Aktivierung des trigeminovaskulären Systems mit Freisetzung vasoaktiver Peptide kommt, die neben einer Vasodilatation auch weitere Vorgänge in Gang setzen (Limmroth und Diener 2003). Anschließend kommt es zu einer Aktivierung zentraler, sekundärer trigeminaler Neurone, die über den Thalamus zum primär sensorischen Kortex weitergeleitet wird, welches zur Wahrnehmung des Schmerzes führt. Hier bleibt aber die entscheidende Frage, wie es zur Initialisierung einer neurogenen Entzündung und Aktivierung des trigeminovaskulären Systems kommt, offen. 
Neben den 0.g. peripheren Entstehungsmechanismen gibt es auch eine Reihe von Studienhinweisen für zentrale Entstehungsmechanismen. Hier spielt der Hirnstamm eine führende Rolle als „Migränegenerator“. Während einer Migräneattacke konnte beim Menschen eine deutliche Aktivierung unterschiedlicher Areale im Hirnstammbereich gesehen werden (Weiller et al. 1995):

1. Das exzitatorische nozizeptive System im trigeminalen Nucleus caudalis,

2. Das inhibitorische absteigende Schmerzhemmsystem mit Beteiligung des periaquäduktalen Graus (PAG) bzw. der Raphekerne sowie des segmentalen inhibitorischen Systems,

3. Der Locus coeruleus und

4. Unabhängig von Lateralität die Area postrema mit angrenzendem Nucleus solitarius, die für vegetative Begleitsymptome, wie Übelkeit und Erbrechen, verantwortlich gemacht werden können.

Die Verbindung zwischen peripheren Ereignissen (neurogene Entzündung, Durchblutungsänderung) und der postulierten Generatorregion ist bislang ungeklärt (Ebersberg 2002). Gleichzeitig bewirkt die Aktivierung der Hirnstammneurone auch eine Aktivierung von umliegenden zentralen Kontroll- und Steuerungsmechanismen. Hierdurch können z.B. die vegetativen Begleitsymptome und die Nackenverspannungen während der Migräneattacke erklärt werden.

Zur Entstehung der Migränekopfschmerzen spielt der Serotoninhaushalt auch eine wichtige Rolle. Serotonin könnte aus dem Endothel der Blutgefäße durch Aktivierung lokaler 5- $\mathrm{HT}_{2 \mathrm{~B}}$-Rezeptoren Stickstoffoxid (NO) freisetzen, das gefäßbegleitende nozizeptive Afferenzen aktiviert. Zum anderen könnte ein Mangel an Serotonin dazu führen, dass die tonische inhibierende Wirkung einer $5-\mathrm{HT}_{1 \mathrm{~B} / \mathrm{D}-}$ Rezeptoraktivierung auf trigeminale nozizeptive Afferenzen und nachgeschaltete Neurone vermindert wird oder entfällt. Beide Vorgänge würden zu einer verstärkten Aktivierung des trigeminovaskulären Systems führen. Als Auslöser für Migränekopfschmerzen könnte also sowohl zu wenig als auch zu viel Serotonin eine Rolle spielen (Ebersberg 2002).

Serotonin wird in der Dura aus den perivaskulären sympathischen Fasern aufgenommen und dort gespeichert (Stanley et al. 1993). Die Freisetzung von Serotonin in der Dura ist von der Stimulation des sympathischen Nervensystems abhängig. Aber auch eine elektrische Reizung parasympathischer Fasern in der Ratte (Sphenopalatines Ganglion) führte zu einer Plasmaextravasation als Zeichen einer neurogenen Entzündung (Delepine und Aubineau 1997). Bei der Migräne kann das autonome Nervensystem entsprechend die nozizeptiven Afferenzen beeinflussen. Jedoch wird diese Beteiligung des autonomen Nervensystems bei der Migräneentstehung kontrovers diskutiert (Thomson und Olesen 1995). 
Zur Entstehung der Migräne ist bereits seit längerem eine erbliche Disposition einer besonderen Form der Migräne (sog. Familiäre Hemiplegische Migräne) belegt (Ophoff et al. 1997). Es handelt sich dabei um Veränderungen in einem spannungsabhängigen Kalziumkanal und in einem Kaliumkanal, die auf Chromosom 19p13 und 1q23 kodiert und vor allem im zentralen Nervensystem exprimiert werden. Dabei müssen neben den o.g. noch weitere Genloci beteiligt sein. Die häufigsten Formen der vererbten Migräne weisen sicherlich eine polygenetische Vererbung mit unvollständiger Penetranz auf (May et al. 1995, Nyholt et al. 1998).

Auf Grundlage dieser genetischen Veränderung in den Kalziumkanälen kommt es durch Triggerreize oder Triggerfaktoren, die die Aktivierungszustände dieser Kanäle beeinflussen und möglicherweise zum Auslösen von Attacken notwendig sind, zu einem Fehler in der Freisetzung inhibitorischer Transmitter, der entweder zu einer veränderten kortikalen Erregbarkeit oder zu einer gestörten Inhibition des nozizeptiven Systems führt. Damit könnte das attackenhafte Auftreten der Migräne erklärt werden (Ebersberg 2002).

\subsubsection{Therapie}

Die folgenden Aussagen stützen sich im Wesentlichen auf das Review von Evers et al. (2008) und die EFNS-Empfehlungen zur medikamentösen Behandlung der Migräne (Evers et al. 2006).

Die Behandlung einer Migräneattacke wird in klinischen Studien als erfolgreich bezeichnet, wenn folgende Kriterien erfüllt sind (May 2006):

- das Fehlen von Kopfschmerzen zwei Stunden nach Behandlung oder

- die Besserung der Kopfschmerzen von schwer oder mittelschwer auf leicht oder kopfschmerzfrei innerhalb von zwei Stunden nach Applikation des entsprechenden Präparates

- eine reproduzierbare Wirkung bei zwei von drei Migräneattacken

- das Fehlen von Kopfschmerzen und keine Einnahme von Medikamenten innerhalb von 24 Stunden nach erfolgreicher Therapie.

Wenn man diese Maßstäbe anlegt, ergibt sich nach evidenzbasierten Kriterien die folgende Zusammenfassung der wichtigsten Empfehlungen zur Akuttherapie und Prophylaxe der Migräne (s. Tabelle 2 nach Evers et al. (2008)). 
Tabelle 2: Zusammenfassung der wichtigsten Empfehlungen zur Akuttherapie und Prophylaxe der Migräne. $A$ = Hohe Empfehlungsstärke aufgrund starker Evidenz oder bei schwächerer Evidenz aufgrund besonders hoher Versorgungsrelevanz, $\mathrm{B}=$ Mittlere Empfehlungsstärke aufgrund mittlerer Evidenz oder bei schwacher Evidenz mit hoher Versorgungsrelevanz oder bei starker Evidenz und Einschränkungen der Versorgungsrelevanz, $\mathrm{C}$ = Niedrige Empfehlungsstärke aufgrund schwächerer Evidenz oder bei höherer Evidenz mit Einschränkungen der Versorgungsrelevanz (modifiziert nach Evers et al. 2008, S.934).

- $\quad$ Die 5-HT1B/1D-Agonisten (in alphabetischer Reihenfolge) Almotriptan, Eletriptan, Frovatriptan, Naratriptan, Rizatriptan, Sumatriptan und Zolmitriptan sind die Substanzen mit der besten Wirksamkeit bei akuten Migräneattacken (A).

- $\quad$ Nichtopioidanalgetika und nicht steroidale Antirheumatika sind bei der Behandlung der Migräne wirksam (A).

- $\quad$ Ergotamin ist bei Migräne wirksam. Allerdings ist die Wirksamkeit in prospektiven Studien schlecht belegt (B).

- $\quad$ Die Wirksamkeit nicht medikamentöser Verfahren wurde in der Attackentherapie in kontrollierten Studien kaum untersucht $(\mathrm{C})$.

- $\quad$ Bei häufigen Migräneattacken bzw. Migräneattacken mit ausgeprägten Beschwerden oder neurologischen Ausfällen sollte eine Migräneprophylaxe begonnen werden (A).

- $\quad$ Migräneprophylaktika der ersten Wahl sind Metoprolol (A), Propranolol (A), Flunarizin (A), Topiramat (A) und Valproinsäure (noch Off-label) (A).

- $\quad$ Migräneprophylaktika der zweiten Wahl sind Bisoprolol (B), Naproxen (B), Vitamin B2 (B), Pestwurz (B) Amitriptylin (B), Lisinopril (C), Candesartan (C), Gabapentin (C), Acetylsalicylsäure (C), Magnesium (C), Coenzym Q10 (C).

- $\quad$ Die Prophylaxe von Migräneauren im Rahmen einer Migräne mit Aura kann mit Lamotrigin erfolgen (C)

- $\quad$ Die Akupunktur ist unabhängig von der durchgeführten Form vergleichbar wirksam mit einer

Scheinakupunktur oder einer medikamentösen Prophylaxe (A).

- $\quad$ Die medikamentöse Therapie sollte durch nicht medikamentöse Verfahren, der Verhaltenstherapie (A) und durch Ausdauersport (B) ergänzt werden. Alternativ zur medikamentösen Therapie kann auch eine Verhaltenstherapie als Prophylaxe durchgeführt werden $(A)$.

- $\quad$ Bei Patienten mit einer hochfrequenten Migräne (> 3 Attacken/Monat) sowie erheblicher Einschränkung der Lebensqualität sollte eine psychologische Therapie angestrebt werden (A). Es besteht ein erhöhtes Risiko der Chronifizierung.

\subsubsection{Medikamentöse Akuttherapie}

\subsection{Substanzen zur Akuttherapie}

\subsection{Analgetika und Antiemetika}

Akute Migräneattacken, die leicht- bis mittelgradig in ihrer Schmerzintensität sind, sollten in erster Linie mit sogenannten peripher wirksamen Analgetika bzw. nicht steroidalen Antirheumatika (NSAR) behandelt werden (Tabelle 3). Da die meisten Patienten während der Migräneattacke unter gastrointestinalen Symptomen leiden, sollten Antiemetika zur Bekämpfung der Übelkeit und zur Steigerung der Resorptionsfähigkeit ca. 10 Minuten vor Einnahme der Analgetika eingenommen werden. Hier werden Metoclopramid und Domperidon empfohlen (Tabelle 4). Anschließend sollte die hochdosierte und frühzeitige Gabe von Acetylsalicylsäure (ASS), Paracetamol, Ibuprofen, Diclofenac oder Naproxen erfolgen. Wahrscheinlich sind auch Metamizol und Phenazon wirksam. Es gibt bis heute keine Erkenntnisse darüber, welche dieser Substanzen am effektivsten eingesetzt wird. In der konkreten Empfehlung für einen Patienten sollte man sich neben der subjektiven Wirksamkeit und 
Präferenz durch den Patienten auch an dem Nebenwirkungsprofil und den Applikationsmöglichkeiten

(z.B. rektal, nasal, Brausetablette, Kautablette) der einzelnen Substanzen orientieren.

\begin{tabular}{|c|c|c|c|}
\hline & & & Kontraindikationen \\
\hline $\begin{array}{l}\text { Acetylsalicylsäure } \\
\text { (Aspirin®) } \Uparrow \Uparrow \\
\text { ASS-lysinat (AspirinIV®) }\end{array}$ & $\begin{array}{l}1000 \mathrm{mg} \\
1000 \mathrm{mg} \text { i. v. }\end{array}$ & $\begin{array}{l}\text { Magenschmerzen, Übelkeit, } \\
\text { Gerinnungsstörungen, } \\
\text { allergische Reaktionen }\end{array}$ & $\begin{array}{l}\text { Magen-Darm-Ulzera, } \\
\text { Hämorrhagische Diathese, } \\
\text { Schwangerschaft Monat } 7 \text { bis } \\
9\end{array}$ \\
\hline Ibuprofen (Aktren $®) ~ \Uparrow \Uparrow$ & 200 bis $600 \mathrm{mg}$ & wie ASS, Ödeme & $\begin{array}{l}\text { wie ASS (Blutungsneigung } \\
\text { geringer), Niereninsuffizienz, } \\
\text { Lupus erythematodes }\end{array}$ \\
\hline Naproxen (Proxen®) $\Uparrow \Uparrow$ & $500-1000 \mathrm{mg}$ & wie Ibuprofen & wie Ibuprofen \\
\hline Diclofenac (Voltaren $®) \Uparrow \Uparrow$ & 50 bis $100 \mathrm{mg}$ & wie Ibuprofen & wie Ibuprofen \\
\hline Metamizol (Novalgin®) $\Uparrow$ & $1000 \mathrm{mg}$ & $\begin{array}{l}\text { Allergische Reaktion, } \\
\text { Blutbildveränderungen, } \\
\text { bei i.v.-Gabe Hypotonie }\end{array}$ & $\begin{array}{l}\text { Erkrankungen des } \\
\text { hämatopoetischen Systems }\end{array}$ \\
\hline $\begin{array}{l}\text { Paracetamol (ben-u-ron®) } \\
\Uparrow\end{array}$ & $1000 \mathrm{mg}$ & Leberschäden & $\begin{array}{l}\text { Leberschäden, } \\
\text { Niereninsuffizienz }\end{array}$ \\
\hline $\begin{array}{l}\text { ASS plus Paracetamol + } \\
\text { Koffein (Thomapyrin®) } \Uparrow \Uparrow\end{array}$ & $250+200+50 \mathrm{mg}$ & wie ASS und Paracetamol & wie ASS und Paracetamol \\
\hline
\end{tabular}

Tabelle 4: Antiemetika in der Migränetherapie. $\Uparrow \Uparrow=$ Aussage zur Wirksamkeit wird gestützt durch mehrere adäquate, valide klinische Studien bzw. durch eine oder mehrere valide Metaanalysen oder systematische Reviews. Positive Aussage gut belegt. $\Leftrightarrow=$ Es liegen keine sicheren Studienergebnisse vor, die eine günstige oder ungünstige Wirkung belegen. Dies kann bedingt sein durch das Fehlen adäquater Studien, aber auch durch das Vorliegen mehrerer, aber widersprüchlicher Studienergebnisse (modifiziert nach Evers et al. 2008, S. 936).

\begin{tabular}{|c|c|c|c|}
\hline Substanzen & Dosis & Nebenwirkungen & Kontraindikationen \\
\hline $\begin{array}{l}\text { Metoclopramid } \Uparrow \Uparrow \\
\text { (z. B. Paspertin } ®)\end{array}$ & $\begin{array}{l}10 \text { bis } 20 \text { mg p.o. } \\
20 \text { mg Supp. } \\
10 \text { mg i.m., i.v., s.c. }\end{array}$ & $\begin{array}{l}\text { frühes dyskinetisches } \\
\text { Syndrom, } \\
\text { Unruhezustände }\end{array}$ & $\begin{array}{l}\text { Kinder unter } 14 \text { Jahren, } \\
\text { Hyperkinesen, Epilepsie } \\
\text { Schwangerschaft, } \\
\text { Prolaktinom }\end{array}$ \\
\hline Domperidon (Motilium ${ }^{\circledR}$ ) $\Leftrightarrow$ & 20 bis $30 \mathrm{mg} \mathrm{p.o.}$ & $\begin{array}{l}\text { seltener als bei } \\
\text { Metoclopramid }\end{array}$ & $\begin{array}{l}\text { Kinder unter } 10 \text { Jahren, } \\
\text { sonst siehe Metoclopramid, } \\
\text { aber geringer ausgeprägt } \\
\text { und seltener }\end{array}$ \\
\hline
\end{tabular}

Wenn die sogenannten peripheren Analgetika nicht wirksam sind oder wenn es sich um schwere Migräneattacken handelt (und das ist bei ca. einem Drittel der Migränepatienten, vor allem z.B. bei menstruellen Migräneattacken der Fall), ist der nächste Schritt die Gabe eines Triptans. Kontraindikationen dagegen sind manifeste Herz-Kreislauf-Erkrankungen. Eine Übersicht über die zur 
Verfügung stehenden Substanzen in der akuten Migränetherapie, wie sie von der DMKG empfohlen wird, gibt Tabelle 5.

Es wird empfohlen, die Einnahme von Akutmedikamenten gegen Migräne auf maximal zehn Tage pro Monat und auf maximal drei Tage hintereinander zu beschränken, da alle Analgetika bei zu häufiger regelmäßiger Einnahme zu einem Kopfschmerz bei Medikamentenübergebrauch führen können.

Tabelle 5: Therapie der akuten Migräneattacke mit 5-HT1B/1D-Agonisten (Reihenfolge nach dem Jahr der Zulassung). $\Uparrow \Uparrow=$ Aussage zur Wirksamkeit wird gestützt durch mehrere adäquate, valide klinische Studien bzw. durch eine oder mehrere valide Metaanalysen oder systematische Reviews. Positive Aussage gut belegt. $\Uparrow=$ Aussage zur Wirksamkeit wird gestützt durch zumindest eine adäquate, valide klinische Studie. Positive Aussage belegt. $\Leftrightarrow=$ Es liegen keine sicheren Studienergebnisse vor, die eine günstige oder ungünstige Wirkung belegen. Dies kann bedingt sein durch das Fehlen adäquater Studien, aber auch durch das Vorliegen mehrerer, aber widersprüchlicher Studienergebnisse * Bei Unwirksamkeit von $40 \mathrm{mg}$ können auch $80 \mathrm{mg}$ Eletriptan auf einmal gegeben werden, dann etwas stärkere Nebenwirkungen als Sumatriptan (modifiziert nach Evers et al. 2008, S. 936)

\begin{tabular}{|c|c|c|c|}
\hline Substanzen & Dosis & Nebenwirkungen & Kontraindikation \\
\hline $\begin{array}{l}\text { Sumatriptan } \Uparrow \Uparrow \\
\text { (Imigran®, } \\
\text { Imigran®T) }\end{array}$ & $\begin{array}{l}50 \text { bis } 100 \mathrm{mg} \mathrm{p.o.} \\
25 \mathrm{mg} \text { Supp. } \\
10 \text { bis } 20 \mathrm{mg} \text { nasal } \\
6 \mathrm{mg} \text { s.c. (Autoinjektor) }\end{array}$ & $\begin{array}{l}\text { Engegefühl im } \\
\text { Bereich der Brust } \\
\text { und des Halses, } \\
\text { Parästhesien der } \\
\text { Extremitäten, } \\
\text { Kältegefühl, } \\
\text { Lokalreaktion an } \\
\text { der Injektionsstelle }\end{array}$ & $\begin{array}{l}\text { Hypertonie, koronare Herzerkrankung, } \\
\text { Angina pectoris, Myokardinfarkt in der } \\
\text { Vorgeschichte, M. Raynaud, periphere } \\
\text { arterielle Verschlusskrankheit, TIA oder } \\
\text { Schlaganfall, Schwangerschaft, Stillzeit, } \\
\text { Kinder (< } 12 \text { Jahre), schwere Leber- oder } \\
\text { Niereninsuffizienz, multiple vaskuläre } \\
\text { Risikofaktoren, gleichzeitige Behandlung } \\
\text { mit Ergotamin, innerhalb von zwei } \\
\text { Wochen nach Absetzen eines MAO- } \\
\text { Hemmers }\end{array}$ \\
\hline $\begin{array}{l}\text { Zolmitriptan } \\
\text { (AscoTop } ®) \Uparrow \Uparrow\end{array}$ & $\begin{array}{l}2,5 \text { bis } 5 \mathrm{mg} \mathrm{p} \text { p.o. oder } \\
\text { als Schmelztablette, } 5 \\
\text { mg nasal }\end{array}$ & wie Sumatriptan & wie Sumatriptan \\
\hline $\begin{array}{l}\text { Naratriptan } \Uparrow \Uparrow \\
\text { (Naramig®, } \\
\text { Formigran } ® \text { ) }\end{array}$ & 2,5 mg p.o. & $\begin{array}{l}\text { etwas geringer als } \\
\text { Sumatriptan }\end{array}$ & wie Sumatriptan \\
\hline $\begin{array}{l}\text { Rizatriptan } \\
(\text { Maxalt@ } ₫) \Uparrow\end{array}$ & $\begin{array}{l}10 \mathrm{mg} \text { p.o. oder als } \\
\text { Schmelztablette }\end{array}$ & wie Sumatriptan & $\begin{array}{l}\text { wie Sumatriptan; Dosis } 5 \mathrm{mg} \text { bei } \\
\text { gleichzeitiger Einnahme von Propranolol }\end{array}$ \\
\hline $\begin{array}{l}\text { Almotriptan } \\
(\text { Almogran } \circledast) ~ \Uparrow \Uparrow\end{array}$ & 12,5 mg p.o. & $\begin{array}{l}\text { etwas geringer als } \\
\text { Sumatriptan }\end{array}$ & wie Sumatriptan \\
\hline $\begin{array}{l}\text { Eletriptan* } \\
(\text { Relpax囚) } \Uparrow \Uparrow\end{array}$ & 20 bis $40 \mathrm{mg}$ p.o. & wie Sumatriptan & wie Sumatriptan \\
\hline $\begin{array}{l}\text { Frovatriptan } \\
\text { (Allegro®) } \Uparrow \Uparrow\end{array}$ & 2,5 mg p.o. & $\begin{array}{l}\text { etwas geringer als } \\
\text { Sumatriptan }\end{array}$ & wie Sumatriptan \\
\hline
\end{tabular}

\subsection{5-HT1B/1D-Agonisten (Triptane)}

Die Wahl des Triptans zur Behandlung schwerer Migräneattacken richtet sich nach der Applikationsform (oral, nasal, rektal, subkutan, Schmelztablette) und nach den Erfahrungen des Patienten, weniger nach den pharmakologischen Eigenschaften der jeweiligen Substanz. Die Ähnlichkeiten der Triptane in Bezug auf gute Wirksamkeit und geringe Nebenwirkungen überwiegen die Unterschiede in ihrer 
Pharmakologie bei weitem. Derzeit sind in Deutschland 7 Triptane erhältlich (Tabelle 5) (in der

Reihenfolge ihrer Zulassung: Sumatriptan, Zolmitriptan, Naratriptan, Rizatriptan, Almotriptan, Eletriptan, Frovatriptan). Prinzipiell sollten Triptane nicht an mehr als 10 Tagen im Monat eingenommen werden. Naratriptan und Frovatriptan unterscheiden sich von den übrigen Triptanen durch einen etwas verzögerten Wirksamkeitseintritt, durch eine etwas geringere Wirksamkeit, aber auch durch eine längere Wirksamkeitsdauer. Die übrigen Triptane sind sich in ihrer Wirksamkeit so ähnlich, dass keine sichere Empfehlung für die erste Wahl gegeben werden kann. Eine Ausnahme stellt nur das subkutane Sumatriptan dar, welches am wirksamsten ist, aber auch eine hohe Wiederkehrrate der Kopfschmerzen aufweist. Es gibt Patienten, die auf bestimmte Triptane besser ansprechen als auf andere, so dass es sinnvoll sein kann, bei Versagen eines Triptans, andere auszuprobieren.

\subsection{Mutterkornalkaloide}

Obwohl Triptane im Vergleich zu Mutterkornalkaloiden besser wirksam sind, sollte die Behandlung mit Ergotamintartrat sehr langen Migräneattacken oder solchen mit mehrfacher Wiederkehr (sogenannter Recurrence) vorbehalten bleiben. Patienten, die ihre Migräneattacke erfolgreich mit einem Mutterkornalkaloid behandeln und keine Nebenwirkungen oder keine Dosissteigerung haben, können diese Akuttherapie beibehalten. Die gehäufte Einnahme von Ergotamintartrat kann auch zu einem Kopfschmerz bei Medikamentenübergebrauch führen. Daher muss die Einnahmefrequenz auf maximal zehn Tage pro Monat und an maximal drei aufeinander folgenden Tagen begrenzt werden. In epidemiologischen Studien ist das Risiko vaskulärer Ereignisse bei der Einnahme von Mutterkornalkaloiden erhöht. Das ist bei den Triptanen nicht der Fall. Das einzige in Deutschland zur Behandlung der akuten Migräneattacke zugelassene Ergotaminpräparat enthält Ergotamintartrat (Ergo Kranit akut@) (Tabelle 6).

\begin{tabular}{|c|c|c|c|}
\hline Substanz & Dosis & Nebenwirkungen & Kontraindikationen \\
\hline $\begin{array}{l}\text { Ergotamintartrat } \\
\text { (Ergo Kranit } \\
\text { akut@) }\end{array}$ & $2 \mathrm{mg}$ p.o. & $\begin{array}{l}\text { Erbrechen, Übelkeit, } \\
\text { Kältegefühl, } \\
\text { Muskelkrämpfe, } \\
\text { Dauerkopfschmerz } \\
\text { und Ergotismus }\end{array}$ & $\begin{array}{l}\text { Schwangerschaft, Stillzeit, Kinder unter } 12 \text { Jahren, } \\
\text { Patienten mit multiplen vaskulären Risikofaktoren, } \\
\text { schlecht eingestellte Hypertonie, koronare } \\
\text { Herzerkrankung, Angina pectoris, Myokardinfarkt in } \\
\text { der Vorgeschichte, M. Raynaud, periphere arterielle } \\
\text { Verschlusskrankheit, TIA oder Schlaganfall, schwere } \\
\text { Leber- oder Niereninsuffizienz und multiple vaskuläre } \\
\text { Risikofaktoren. }\end{array}$ \\
\hline
\end{tabular}




\subsection{Andere Substanzen}

Die intravenöse Gabe von Valproinsäure ist zwar in einer Dosis von 300 bis 800 mg in der Behandlung akuter Migräneattacken wirksam, aber noch ist sie zur Behandlung von Migräneattacken nicht zugelassen. Tramadol hat in der Kombination mit Paracetamol eine Wirksamkeit bei akuten Migräneattacken gezeigt. Opioide und Tranquilizer sollten wegen begrenzter Wirksamkeit, häufigem Erbrechen und einem hohen Suchtpotenzial dennoch nicht zur Behandlung der Migräneattacke eingesetzt werden. Eine neue Substanzgruppe sogenannter „CGRP-Antagonisten“ befinden sich im Moment in der klinischen Erprobung. Sie haben den Vorteil, keine Vasokonstriktion hervorzurufen. Die ersten Ergebnisse hierzu sind positiv (Ho et al. 2008, Olesen et al. 2004).

\subsection{Behandlung von Migräneattacken als Notfall}

Im ärztlichen Notdienst ist die Behandlung der ersten Wahl die intravenöse Gabe von 1000 mg ASS mit oder ohne Metoclopramid. Liegen keine Kontraindikationen vor, kann auch 6 mg Sumatriptan subkutan gegeben werden. Alternativ kann auch 1000 mg Metamizol i.v. gegeben werden, dies jedoch mit großer Vorsicht wegen der Gefahr eines Blutdruckabfalls und allergischer Reaktionen. Im Status migraenosus (d.h. Migräneattacken, die länger als drei Tage andauern) erfolgt die Therapie durch einmalige Gabe von 50 bis $100 \mathrm{mg}$ Prednisolon oder $10 \mathrm{mg}$ Dexamethason. Die intravenöse Gabe von Paracetamol bei akuten Migräneattacken war in einer placebo-kontrollierten Studie nicht wirksam (Leinisch et al. 2005).

\subsubsection{Medikamentöse Migräneprophylaxe}

\subsection{Grundlagen}

Es liegen keine evidenzbasierten Kriterien für die exakte Indikationsstellung zur Migräneprophylaxe vor. Nach der Leitlinie der Deutschen Migräne- und Kopfschmerzgesellschaft und der Deutschen Gesellschaft für Neurologie (Evers et al. 2008) wird eine Migräneprophylaxe insbesondere dann empfohlen, wenn einer der folgenden Punkte vorliegt:

- Drei und mehr Migräneattacken pro Monat.

- Migräneattacken, die regelmäßig länger als 72 Stunden anhalten.

- Attacken, die auf eine Therapie entsprechend den gegebenen Empfehlungen (inkl. Triptanen) nicht ansprechen und/oder wenn Nebenwirkungen der Akuttherapie nicht toleriert werden.

- Bei Zunahme der Attackenfrequenz und Einnahme von Schmerz- oder Migränemitteln an mehr als zehn Tagen im Monat. 
- Bei komplizierten Migräneattacken mit beeinträchtigenden (z.B. hemiplegischen) und/oder lang anhaltenden Auren.

- Zustand nach migränösem Infarkt nach Ausschluss anderer Ursachen.

Ziel der medikamentösen Migräneprophylaxe ist eine Reduzierung von Häufigkeit, Schwere und Dauer der Migräneattacken und die Prophylaxe des Kopfschmerzes bei Medikamentenübergebrauch. Von einer Wirksamkeit einer Migräneprophylaxe spricht man bei einer Senkung der Attackenfrequenz von mindestens $50 \%$.

Nach Konsens der Expertengruppe der deutschen Migräne- und Kopfschmerzgesellschaft sowie der Deutschen Gesellschaft für Neurologie (Evers et al. 2008) wird das folgende grundsätzliche Vorgehen bei einer medikamentösen Prophylaxe der Migräne empfohlen:

- Da die meisten Nebenwirkungen in den ersten Wochen der Einnahme auftreten, sollen die Medikamente grundsätzlich schrittweise aufdosiert werden.

- Die meisten Medikamente zur Migräneprophylaxe können zu Müdigkeit als Nebenwirkung führen, sie sollten daher abends eingenommen werden.

- Da alle prophylaktischen Medikamente ursprünglich eine andere Indikation haben als die Migräneprophylaxe, ist eine eingehende Aufklärung der Patienten zur Erhaltung der Compliance sehr wichtig.

- Aufgrund der Pharmakodynamik der meisten Medikamente zur Migräneprophylaxe kann erst nach sechs bis acht Wochen die Wirksamkeit beurteilt werden. Bis dahin sollten die Medikamente allenfalls aufgrund von Nebenwirkungen abgesetzt werden. Zur Beurteilung der Wirksamkeit soll ein Kopfschmerzkalender geführt werden.

- Eine wirksame medikamentöse Prophylaxe sollte wenigstens sechs Monate lang gegeben werden, dann ist ein Auslassversuch sinnvoll, um zu überprüfen, ob noch eine Indikation für die Prophylaxe besteht. In Einzelfällen kann es sinnvoll sein, die Prophylaxe in Absprache mit dem Patienten über diesen Zeitraum hinaus zu geben.

\subsection{Substanzen zur Migräneprophylaxe}

\subsection{Substanzen der ersten Wahl}

Seit vielen Jahren haben sich als Mittel der ersten Wahl die Betablocker Propranolol und Metoprolol, der Kalziumkanalblocker Flunarizin sowie in den letzten Jahren die Antikonvulsiva Valproinsäure und Topiramat etabliert. Tabelle 7 zeigt Dosierung und spezielle Nebenwirkungen von Substanzen zur Migräneprophylaxe. 


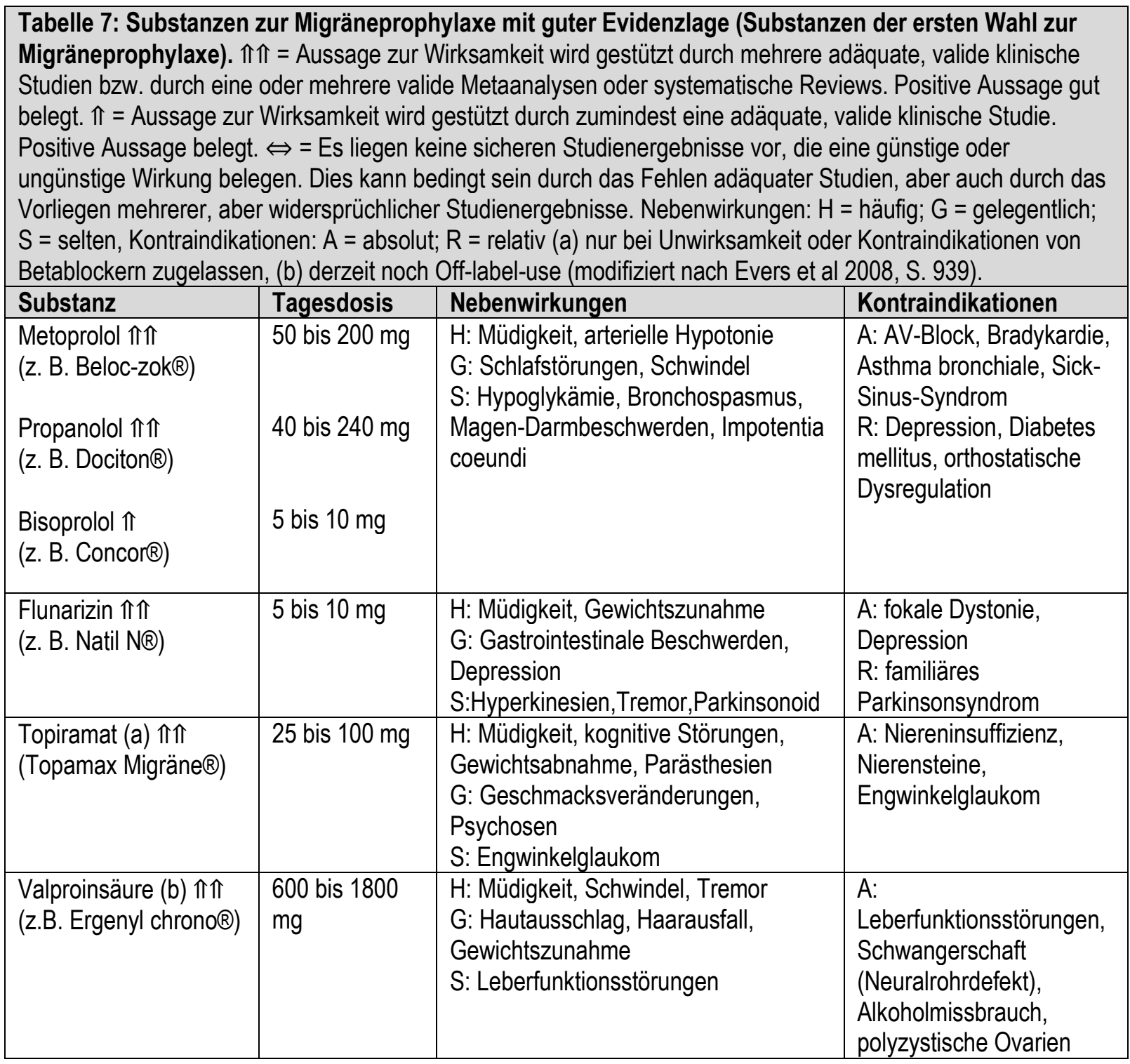

\section{Betablocker}

Propranolol und Metoprolol sind von den Betablockern am besten erforscht, sie sollten mit einem abendlichen Schwerpunkt gegeben werden, bei vielen Patienten genügt eine einmalige Dosis am Tag (in retardierter Form). Das Hauptproblem der Betablocker ist, dass sie bei bestimmten Bedingungen wie arterielle Hypotonie, Bradykardie oder Asthma bronchiale nicht gegeben werden dürfen. Die Patienten beklagen am häufigsten eine Zunahme von Müdigkeit, Gewichtszunahme und seltener Schlafstörungen (Albträume, fraktionierter Schlaf).

\section{Kalziumkanalblocker (KKB)}

Ebenfalls Mittel der ersten Wahl ist Flunarizin, der einzige zugelassene KKB in der Migräneprophylaxe. Flunarizin weist nicht die kardiovaskulären und pulmonalen Kontraindikationen auf wie Betablocker. 
Allerdings treten neben den für die Betablocker typischen Nebenwirkungen noch in seltenen Fällen Depressionen oder extrapyramidale Bewegungsstörungen auf.

\section{Antiepileptika}

In jüngerer Zeit sind Antiepileptika in den Mittelpunkt des klinischen Forschungsinteresses gerückt. Topiramat ist mittlerweile für die Prophylaxe der Migräne in Deutschland zugelassen und zählt zu den Mitteln der ersten Wahl. Die ansonsten beste Datenlage existiert für Valproat, das in seiner Wirksamkeit nach den Kriterien der EBM mit den Betablockern und Flunarizin vergleichbar ist, und mittlerweile als Mittel der ersten Wahl empfohlen wird, eine Zulassung für die Migräneprophylaxe besteht neuerdings als Off-label-Therapie. Limitierend sind neben den typischen Nebenwirkungen wie Gewichtszunahme, Müdigkeit und Tremor die selten auftretenden Leberenzymerhöhungen und die dermatologischen Probleme (allergisches Exanthem, Haarausfall). Für eine Reihe von weiteren Substanzen aus der Gruppe der Antiepileptika (u.a. Gabapentin) liegen ebenfalls mehrere positive Studien vor, die jedoch noch nicht ausreichend repliziert sind.

\subsection{Substanzen der zweiten Wahl}

Für weitere Substanzen liegen weniger kontrollierte Studien vor oder ist die Wirksamkeit nicht so ausgeprägt wie bei den zuvor genannten. Diese Substanzen sollten daher nur eingesetzt werden, wenn die Mittel der ersten Wahl nicht wirksam gewesen sind oder wenn gegen alle Kontraindikationen vorliegen. Zu diesen Substanzen der zweiten Wahl gehören Amitriptylin, Naproxen, ASS, Gabapentin, Pestwurz und Magnesium. Weiterhin gibt es dann noch Substanzen wie Pizotifen, Methysergid, Mutterkraut und Lisinopril, deren Stellenwert nicht sicher geklärt ist, für die es jedoch wenigstens eine positive kontrollierte Studie gibt. Diese spielen aber in der täglichen Praxis so gut wie keine Rolle. Eine weitere neu erforschte Substanz ist Riboflavin (Vitamin B2), welches allerdings in sehr hohen täglichen Dosen (400 mg) eingenommen werden muss. Neu positiv getestet war auch Coenzym Q10 (3x100mg pro Tag) (Sandor et al. 2005). Eine Übersicht über die verschiedenen Substanzen der zweiten Wahl zur Migräneprophylaxe gibt Tabelle 8. 


\begin{tabular}{|c|c|c|c|}
\hline \multicolumn{4}{|c|}{$\begin{array}{l}\text { Tabelle 8: Substanzen zur Migräneprophylaxe mit weniger guter Evidenzlage (Substanzen der zweiten } \\
\text { Wahl zur Migräneprophylaxe). } \Uparrow=\text { Aussage zur Wirksamkeit wird gestützt durch zumindest eine adäquate, } \\
\text { valide klinische Studie. Positive Aussage belegt. } \Leftrightarrow=\text { Es liegen keine sicheren Studienergebnisse vor, die } \\
\text { eine günstige oder ungünstige Wirkung belegen. Dies kann bedingt sein durch das Fehlen adäquater Studien, } \\
\text { aber auch durch das Vorliegen mehrerer, aber widersprüchlicher Studienergebnisse. Nebenwirkungen: } \mathrm{H}= \\
\text { häufig; } G=\text { gelegentlich; } S=\text { selten, Kontraindikationen: } A=\text { absolut; } R=\text { relativ (modifiziert nach Evers et al } \\
\text { 2008, S. 939). }\end{array}$} \\
\hline Substanz & Tagesdosis & Nebenwirkungen & Kontraindikationen \\
\hline $\begin{array}{l}\text { Amitriptylin } \Uparrow \\
\text { (z. B. Saroten ret®) }\end{array}$ & 50 bis $150 \mathrm{mg}$ & $\begin{array}{l}\text { H: Mundtrockenheit, Müdigkeit, } \\
\text { Schwindel, Schwitzen } \\
\text { G: Blasenstörung, innere Unruhe, } \\
\text { Impotentia coeundi }\end{array}$ & $\begin{array}{l}\text { A: Engwinkelglaukom, } \\
\text { Prostatahyperplasie } \\
\text { R: } \\
\text { Herzrhythmusstörungen }\end{array}$ \\
\hline $\begin{array}{l}\text { Venlafaxin } \Uparrow \\
\text { (Trevilor®) }\end{array}$ & 75 bis $150 \mathrm{mg}$ & $\begin{array}{l}\text { H: Müdigkeit, } \\
\text { Konzentrationsstörungen, } \\
\text { S: Impotentia coeundi et generandi, } \\
\text { arterielle Hypertonie }\end{array}$ & $\begin{array}{l}\text { A: schwere arterielle } \\
\text { Hypertonie }\end{array}$ \\
\hline $\begin{array}{l}\text { Gabapentin } \Leftrightarrow \\
(\text { z. B. Neurontin } ®)\end{array}$ & bis $2400 \mathrm{mg}$ & $\begin{array}{l}\text { H: Müdigkeit, Schwindel, } \\
\text { Gewichtszunahme } \\
\text { G: Ataxie, gastrointestinale } \\
\text { Störungen }\end{array}$ & $\begin{array}{l}\text { A: schwere Leber- und } \\
\text { Nierenfunktionsstörungen }\end{array}$ \\
\hline $\begin{array}{l}\text { Naproxon } \Uparrow \\
\text { (z. B. Proxen®) }\end{array}$ & 500 bis $1000 \mathrm{mg}$ & $\mathrm{H}:$ Magenschmerzen & $\begin{array}{l}\text { A: Ulcus ventriculi, } \\
\text { Blutungsneigung, } \\
\text { R: Asthma bronchiale }\end{array}$ \\
\hline $\begin{array}{l}\text { Acetylsalicylsäure } \\
\text { (ASS) } \Leftrightarrow \\
\text { (z. B. Aspirin } 囚) \\
\end{array}$ & $300 \mathrm{mg}$ & $\mathrm{H}$ : Magenschmerzen & wie Naproxen \\
\hline $\begin{array}{l}\text { Pestwurz } \Uparrow \\
\text { (z. B. Petadolex } \circledast)\end{array}$ & $150 \mathrm{mg}$ & $\begin{array}{l}\text { H: Magenschmerzen, Reflux } \\
\text { S: Leberfunktionsstörungen }\end{array}$ & Keine \\
\hline Magnesium $\Leftrightarrow$ & $\begin{array}{l}600 \mathrm{mg}(24 \\
\mathrm{mmol})\end{array}$ & H: Diarrhoe & Keine \\
\hline $\begin{array}{l}\text { Riboflavin (Vitamin B2) } \\
\Leftrightarrow\end{array}$ & $400 \mathrm{mg}$ & H: Gelbfärbung des Urins & Keine \\
\hline
\end{tabular}

\subsubsection{Nicht-medikamentöse Migräneprophylaxe}

Von besonderer Bedeutung für viele Patienten sind die nicht-medikamentösen Verfahren zur Migräneprophylaxe. Dazu gehört zum einen das Vermeiden von Triggerfaktoren (z.B. kein abrupter Kohlenhydratentzug, regelmäßiger Schlaf-Wach-Rhythmus, regelmäßiger Koffeinkonsum). Zum anderen gibt es einige Verfahren, die in kontrollierten Studien ihre Wirksamkeit zur Migräneprophylaxe belegt haben oder für die Metaanalysen von mehreren Studien eine Wirksamkeit nahelegen. Eine positive Evidenz gibt es dabei für die progressive Muskelrelaxation nach Jacobson, das thermale Biofeedback, das Vasokonstriktionstraining, die kognitive Verhaltenstherapie und Sporttherapie (Ausdauerbelastung). Höchste Wirksamkeit wird mit der Kombination solcher Verfahren erzielt (insbesondere Kombination von Biofeedback und Muskelrelaxation). Der Stellenwert der Akupunktur 
wurde in neueren Studien untersucht. Unwirksam in kontrollierten Studien ist die Homöopathie. Im Folgenden sollen die nichtmedikamentösen Verfahren näher erläutert werden.

\subsection{Akupunktur}

Es liegt eine Reihe zum Teil multizentrischer Studien zur Wirksamkeit der Akupunktur bei Migräne vor. Mehrzahl dieser Studien entspricht nicht modernen Anforderungen an klinische Studien. Trotzdem zeigten alle Studien eine Wirksamkeit sowohl der Akupunktur nach klassischen Gesichtspunkten als auch durch die Scheinakupunktur gegenüber Kontrollpatienten, so dass Akupunktur als Ergänzung zur leitlinienorientierten Schmerztherapie gesehen werden kann (Endres et al. 2007). Der Einsatz der Akupunktur in der Migräneprophylaxe wird von der Deutschen Migräne- und Kopfschmerzgesellschaft als Expertenkonsens empfohlen (Evers et al. 2008).

\subsection{Hömöopathie}

Die bisher durchgeführten prospektiven Placebo-kontrollierten Studien ergaben keinen Hinweis auf eine Wirksamkeit der Homöopathie (Walach et al. 1997, Whitmarsh et al. 1997, Ernst 1999, Straumscheim et al. 2000).

\subsection{Sport}

Wissenschaftlich mäßig belegt ist die prophylaktische Wirkung aerober Ausdauerbelastung/ Training/Sport, z.B. Nordic Walking, Jogging, Radfahren, Schwimmen und Gymnastik, mind. 1 x / Woche (Busch und Gaul 2008, Narin et al. 2003).

\subsection{Verhaltenstherapie der Migräne}

Eine medikamentöse Behandlung der Migräne sollte, wenn möglich, durch eine Verhaltenstherapie ergänzt werden. Wissenschaftlich bewiesen sind sogenannte multimodale Therapieverfahren wie kognitive Verhaltenstherapie (KVT), die Techniken der progressiven Muskelrelaxation (nach Jacobson), kognitive Techniken, Stress- und Reizverarbeitungstraining und Schmerzbewältigungstechniken (Andrasik 1996 und 2004, Hermann et al. 1995, Holroyd und Penzien 1990, Reid und McGrath 1996). Zahlreiche Studien, dazu gehören Metaanalysen, konnten zeigen, dass sowohl Entspannungsverfahren (meist die progressive Muskelrelaxation nach Jacobson (PMR)) als auch verschiedene Biofeedbackverfahren zu einer Reduktion der Migränehäufigkeit von 35\% bis $45 \%$ führen können (Nestoriuc und Martin 2007, Penzien et al. 2005). Bei der Kombination von Biofeedback mit PMR und 
auch von verhaltenstherapeutischen und pharmakologischen Verfahren sind additive Effekte gegeben (Grazzi et al. 2002, Holroyd et al. 1995). Verhaltenstherapeutische Strategien sollten immer auf der Basis einer systematischen Verhaltensanalyse (z.B. einer speziellen Anamnesetechnik) sowie eines individuell orientierten Genesemodels abgeleitet werden. Die Wirksamkeit (Index aus Intensität und Frequenz der Kopfschmerzen) der einzelnen Therapien und Therapiekombinationen ist Tabelle 9 zu entnehmen.

\begin{tabular}{|c|c|c|}
\hline Therapieverfahren & Verbesserung der Migräneaktivität (\%) & Effektstärke \\
\hline Progressive Muskelrelaxation (PMR) & $32-37$ & 0,55 \\
\hline Thermales Finger-Biofeedback (tBFB) & $35-37$ & 0,38 \\
\hline $\mathrm{PMR}+\mathrm{tBFB}$ & $33-50$ & 0,40 \\
\hline PMR + tBFB + Propranolol & $50-70$ & - \\
\hline Muskuläres Feedback (EMG-BFB) & 40 & 0,77 \\
\hline Kognitiv-behaviorale Therapie (KVT) & $35-49$ & 0,54 \\
\hline $\mathrm{KVT}+\mathrm{tBFB}$ & 38 & 0,37 \\
\hline Placebo-Medikament & $14-30$ & - \\
\hline Keine Behandlung & 2 & - \\
\hline Propranolol & 44 & - \\
\hline
\end{tabular}

\subsection{Andere Verfahren}

Die Wirksamkeit der transkraniellen Gleichstrom- und Magnetstimulation zur Migränebehandlung wird aktuell untersucht, ist jedoch noch nicht ausreichend abschätzbar (Chadaide et al. 2007). Die Wirksamkeit der vielfältigen Methoden der physikalischen Therapie konnte in ihren Anwendungen für die Behandlung der Migräne nachgewiesen werden (Biondi 2005), insbesondere in Kombination mit verhaltenstherapeutischen Interventionen. Es liegen bislang aber nur wenige kontrollierte Studien vor. Bis Dato gibt es nur zwei randomisierte kontrollierte Studien, die die Wirksamkeit der Massage als nichtmedikamentöse Therapie bei der Migräne nachweisen konnten (Lawler und Cameron 2006, Hernandez-Reif et al. 1998). Ebenfalls gibt es nur zwei wissenschaftliche Studien mit geringer Patiententeilnahme (Quinn et al. 2002, Puustjarvi et al. 1990), die die Wirksamkeit der Massage bei chronischem Spannungskopfschmerz zeigten. Als Hinweise, dass Lymphdrainage wirksam gegen Kopfschmerzen vom Spannungstyp ist, gibt es nur eine italienische wissenschaftliche Studie von Longo und Mitarbeiter (Longo et al. 2006), allerdings hier ohne Kontrollgrupe. Ein Hinweis auf Linderung der Kopfschmerzen und Verbesserung der Lebensqualität bei Migränepatienten konnte anhand von drei Einzelfallstudien von Krahl (Krahl 2005) erbracht werden, sonst liegen hierzu keine anderen Studien vor. Für die Behandlung der Migräne mit Botulinumtoxin-A gibt es bisher keine positive Evidenz (Evers et al. 2008). 


\subsection{Verfahren ohne Wirksamkeitsnachweis}

Unwirksam in der medikamentösen Therapie sind Bromocriptin, die Antiepileptika Carbamazepin, Diphenylhydantoin und Primidon, Diuretika, Clonidin, Östrogene und Gestagene, Lithium, Neuroleptika, Proxibarbal und die selektiven Serotonin-Wiederaufnahmehemmer. Zu den nicht-medikamentösen Verfahren, für die kein Wirksamkeitsnachweis in kontrollierten Studien vorliegt, gehören das autogene Training, die chiropraktische Therapie, zervikale Manipulation, Manualtherapie, Zahnextraktion, Aufbissschienen, Gebisskorrektur, Frischzelltherapie, Neuraltherapie, Magnetfeldtherapie, Reizströme, Psychophonie, transkutane elektrische Nervenstimulation (TENS), hyperbare Sauerstofftherapie, Ozontherapie, Tonsillektomie, Fußreflexzonenmassage, Sanierung vermeintlicher Pilzinfektionen des Darmes, Entfernung von Amalgamfüllungen, Hysterektomie, Darmspülung, Durchtrennung des M. corrugator, Augen-Laser- Akupunktur und die klassische Psychoanalyse (Evers et al. 2008). 


\subsection{Klassische Massage (KM)}

Massagen gehören zu den ältesten Behandlungsverfahren überhaupt und sind in allen Kulturen verwurzelt. Sie sind inzwischen zwar in ihrem Wert umstritten, dennoch haben sie auch heute ihre spezifischen Indikationen und Anwendungsmöglichkeiten, die durch andere Methoden nicht ersetzt werden können. Die folgenden Aussagen hierzu stützen sich auf Heisel (2005).

\subsubsection{Definition}

Massage ist eine mechanische Manipulation bzw. Stimulation der Weichteilgewebe (Haut, Unterhautbindegewebe, Muskulatur, Ligamente, Sehnen, Periost), d.h. die Hand ertastet und behandelt einen Befund. In relativ monotoner, sich wiederholender (repetitiver) Weise werden rhythmische DruckZugbeanspruchungen, Streichungen, Reibungen und Dehnungen auf die Körperoberfläche mit dem Ziel, Muskeln, Kreislauf und Nervensystem zu beeinflussen bzw. zu stimulieren, appliziert.

\subsubsection{Physiologische Wirkung}

Experimentelle Studien konnten zeigen, dass verschiedenste physiologische Parameter durch die Massage beeinflusst werden konnten (Walach et al. 1995).

- $\quad$ Rezeptoren in Haut und Unterhaut

- Rezeptoren in der Muskulatur, im Muskelbindegewebe und in den Muskelfaszien

- Gelenkrezeptoren durch kleine oder größere Bewegungen während der Massage

- Zellen: z.B. Mastzellen

- Körperflüssigkeit: Blut, Lymphe, interstitielle Flüssigkeit

- Glatte Muskulatur der Blut- und Lymphgefäße

- Haut, Unterhaut, Körperfaszie und Verschiebeschichten

- Skelettmuskulatur, Sehnen, Sehnengleitgewebe, Insertionszonen, Faszien, Septen, Verschiebeschichten, intramuskuläres Muskelbindegewebe.

Dennoch kann die Wirkung der Massage nicht über eine einheitliche Theorie erklärt werden. Es entstehen physiologische Reaktionen, aus denen sich die Wirkungen der Massage entwickeln, dabei sind folgende Faktoren ausschlaggebend:

- Anwendungsort

- Größe der Berührungsfläche oder der bearbeiteten Fläche

- Kraftaufwand pro Fläche, aus dem sich die Druckstärke ergibt 
- Zeitdauer des mechanischen Reizes

- Häufigkeit innerhalb eines bestimmten Zeitraumes

- Statik oder Dynamik der Reize mit unterschiedlicher Geschwindigkeit.

Diese Faktoren bestimmen die Lokalisation, die Art und Intensität der Vorgänge, die durch Massage ausgelöst werden.

Die Wirkkomponenten der Massage können in mechanische, biochemische, immunologische, neurale und psychische Wirkkomponenten unterschieden werden.

\subsubsection{Mechanische Wirkkomponenten}

Die direkte Wirkung des mechanische Reizes, d.h. der Massagebewegung oder des Massagedruckes, beeinflusst die Körperflüssigkeit, Gefäße und Verschiebeschichten:

- Verschiebung von Körperflüssigkeit, lokale Hyperämie, Druck-Sog-Wirkung

- Beschleunigung des Flüssigkeitstransportes aus dem Gewebe

- Lösung von Adhäsionen in Gleit- und Verschiebegeweben zwischen Haut, Subkutis, Faszien, Muskeln und Bändern.

\subsubsection{Biochemische Wirkkomponenten}

Hier wird der mechanische Reiz in einen biochemischen umgewandelt, indem durch Massage Substanzen im Gewebe freigesetzt oder aus dem Gewebe ausgeschwemmt werden:

- Freisetzung von Substanzen lokal im Gewebe durch Massage führt zu Folgendem:

○ Arterielle Hyperämie

○ Auslösung von nicht-infektiösen Entzündungsreaktionen

○ Schmerzlinderung (eindeutige Nachweise fehlen jedoch noch)

- Abbau von pathologischen strukturellen "Crosslinks" (Querverbindungen) durch Kollagenasen (Carano und Siciliani 1996)

- Ausschwemmung von Substanzen aus dem Gewebe durch Massage führt zu:

○ Schmerzminderung

○ Entmüdung (die Muskulatur wird nach Belastung schneller wieder leistungsfähig).

\subsubsection{Immunologische Wirkkomponenten}

Die klassische Massage hat eine allgemein sedierende und harmonisierende Wirkung auf das Immunsystem (Stabilisierung verschiedener Parameter des Immunsystems, z.B. Interleukin 4, Interleukin 6, Gamma-Interferon) (Werner et al. 1997): 
- Steigerung der unspezifischen Abwehr durch zelluläre Veränderungen

- Verminderung der spezifischen Abwehr, dadurch Verminderung der Überempfindlichkeitsreaktionen

- Verminderung der Stresshormone Adrenalin, Noradrenalin und Kortisol

- Verbesserung der Wundheilung (Kloster 2006).

\subsubsection{Neurale Wirkkomponenten}

Der mechanische Reiz der Massage wird vom peripheren und zentralen Nervensystem aufgenommen, weitergeleitet und verarbeitet und mit physiologischen Reaktionen beantwortet. Damit hat die Massage Einfluss auf Regelkreise, aber auch auf übergeordnete Steuerungssysteme. Diese Wirkkomponenten haben große Bedeutung bei den segmental-regionalen und allgemeinen Fernwirkungen der Massage wie Muskeldetonisierung, Schmerzlinderung, Wirkung auf innere Organe, Angstlösung, „neurovegetative Stabilisierung“ und Entspannung.

\subsubsection{Psychische Wirkkomponenten}

Aus den existierenden Studien zu Wirkungen der Massage auf das Erleben ergeben sich Hinweise auf antidepressive und anxiolytische Effekte (Müller-Oerlinghausen und Berg 2003, Müller-Oerlinghausen et al. 2004, Walach et al. 1995, Weinberg et al. 1988, Field 1998, Field et al. 1992). Die psychosomatische Wirkung der Massage wird über die psychoneuroimmunologische Wirkung auf immunkompetente Zellen der Haut erklärt (Werner et al. 1997).

\subsubsection{Indikationen}

Die Indikationen werden nach Krankheitsgruppen geordnet:

- Degenerativ bedingte Gelenkreizzustände mit reflektorischen Tendomyosen der umspannenden Weichteile (v.a. im gelenknahen periostalen Ansatzbereich), u. a. auch als Voraussetzung für nachfolgende aktive krankengymnastische Bewegungsübungen.

- Degenerative Affektionen, funktionelle und Statikstörungen der Hals- und Rumpfwirbelsäule mit begleitenden (reaktiven) muskulären Dysfunktionen und Dysbalancen,

- Entzündliche (auch rheumatische) Arthritiden (nach Abklingen der akuten Symptomatik),

- Lokale Stoffwechselstörungen (z.B. im Sinne einer Pannikulose) mit ebenfalls reflektorischen muskulären Irritationen,

- Insertionstendopathien als Folge einer lokalen Überlastungsproblematik (für detonisierende Handgriffe bzw. tiefe Friktionen v. a. im Bereich des Schulter- und Kniegelenks), 
- Weichteilrheumatische (oberflächlich lokalisierte) Beschwerdebilder (z.B. Myalgien und Periarthropathien),

- Verletzungsfolgen im Bereich der Muskulatur und der Bänder,

- Bei (Leistungs-) Sportlern im Intervall zwischen aktiven Übungselementen zur Intensivierung der muskulären Erholungsphase,

- Funktionelle Durchblutungsstörung, Venenerkrankungen,

- Kopfschmerzen (nach Schädeltraumen, Migräne, Spannungskopfschmerzen),

- Psychovegetative und psychosomatische Störungen, Stress, psychische Überlastungssyndrome (als begleitende Zusatzmaßnahmen),

- Periphere Nervenläsionen (bei Aussicht auf Reinnervation).

\subsubsection{Kontraindikationen}

- Allgemein:

- Akute fieberhafte Allgemeinerkrankungen,

- Akute Entzündungen innerer Organe,

- Blutungsneigung (z.B. im Rahmen einer Antikoagulantientherapie; Ausnahme: Low- DoseHeparinbehandlung),

- Schwere (dekompensierte) Herz-/Kreislauferkrankungen.

- Lokale:

- (entzündliche) Haut- und/oder Muskelerkrankungen im Behandlungsgebiet (Ausnahme: Sklerodermie, Psoriasis), auch Thrombophlebitiden und Phlebothrombosen,

- ausgeprägte frische Hämatome,

- frische Verletzungen, die einer Immobilisation bedürfen,

- arterielle Verschlusserkrankung im betroffenen Gebiet,

- Morbus Sudeck, keine Massage der betroffenen Extremität,

- Schwere knöcherne Affektionen (Osteomalazie, erhebliche Osteoporose, akute Osteomyelitis), auch periphere Weichteilerkrankungen (z. B. Myositis ossificans),

- $\quad$ (akute) Tendovaginitis, periphere Nervenengpasssyndrome (Karpaltunnelsyndrom u. a.),

- kardiale oder nephrogene Ödeme,

- bösartige Tumoren (im Behandlungsgebiet).

\subsubsection{Technik/ Durchführung}


Überwiegend werden Teilmassage und Großmassage durchgeführt (Rücken, Extremitäten,

Schultergürtel, Beckengürtel).

Bei Durchführung einer Massage sollten die folgenden Faktoren berücksichtigt werden:

- Beginn im gesunden Bereich, je nach Verträglichkeit langsames Heranarbeiten an das gestörte

Gebiet bis zum Maximalpunkt; einschließlich Intensität der Handgriffe,

- Wohltemperierte Behandlungsräume,

- Entspannte Lagerung des Patienten auf der Behandlungsliege und genügend Bewegungsfreiheit für den Therapeuten,

- Eine liegende Körperposition, bei der der Muskulatur jegliche Haltearbeit abgenommen wird, ist zu bevorzugen,

- Ursprung und Ansatz der behandelten Muskulatur sollten weitgehend einander angenähert sein (Mittelstellung des betroffenen Gelenkes; keine Massagebehandlung eines in Dehnstellung befindlichen Muskels),

- Beginn mit (Aus-) Streichungen zur Gewöhnung des Patienten an die manuelle Maßnahme; anschließend Knetungen, Walkungen u. a. mit langsam ansteigendem Massagedruck, der gegen Ende der Behandlung wieder langsam reduziert wird,

- Gleichmäßiger Rhythmus, eher ruhiges Tempo der einzelnen Massagehandgriffe, fließende Übergänge mit beibehaltendem Hautkontakt im Zuge der einzelnen Grifftechnik wichtig; anschließend (Aus-) Streichen,

Gleitmittel (z. B. Massageöl oder spezielle Emulsionen) nur im Fall sehr trockener, spröder oder schuppender Haut, auch bei stark behaarter Haut erforderlich zur Herabsetzung der Reibung (die Haftwirkung der massierenden Hand und damit die therapeutisch gewollte Tiefenwirkung werden bei Einsatz lokaler Externa eher beeinträchtigt). Bei starker Transpiration ist evtl. die Applikation eines trockenen Talkumpuders sinnvoll.

\subsubsection{Massagehandgriffe und ihre Wirkungen}

Zu den grundlegenden Handgriffen zählen:

- Streichung

- Knetung

- Reibung (Friktion bzw. Zirkelung)

- Klopfen

- Vibrationen

- Dehnende Handgriffe. 


\subsection{Streichung "Effleurage“}

- Technik: rhythmisches leichtes Streichen einer größeren Hautoberfläche mit der gesamten inneren Handfläche (große Kontaktfläche) ohne abruptes Loslassen (etwa 5-6 cm/Sekunde); sachter Beginn mit nur allmählicher Steigerung des Druckes während der therapeutischen Sitzung. Ausführungsarten der Streichung sind:

- Ein- oder Beidhandstreichung (abhängig von der Ausdehnung des Muskels)

- Streichung mit beschwerter Hand (beide Hände des Therapeuten übereinander)

- Hand-über-Hand-Streichung (bei großen Muskelflächen am Rücken).

- Effekte: Wirkung lediglich auf die oberflächliche Muskulatur:

- Leichte temporäre Hyperämie bei einer flachen Druckapplikation

- Verbesserung des venösen und lymphatischen Rückstromes bei vermehrtem Druck

- Globale beruhigende und entspannende Wirkung durch Reizung sensibler Nervenendigungen der Haut über das vegetative Nervensystem.

Einsatz v. a. als Vorbereitung und Abschluss der Massage.

\subsection{Knetung „Petrissage“}

- Technik: Durchführung rhythmisch-alternierender S-förmiger Dehnungen, Verwringungen und Ausdrückungen des weich und sanft von seiner Unterlage abgehobenen Gewebes (Haut, Unterhautbinde- und -fettgewebe sowie Muskulatur) quer oder schräg zum muskulären Faserverlauf (etwa 1-2/Sekunde). Bewegungsrichtung von distal nach proximal.

Rollung: Hin- und Herbewegen eines einzelnen Muskels in seiner Längsachse zur Dehnung.

Walkung: lockere großflächige Grifführung mit raschen, schüttelnden Bewegungen (intensiv detonisierend).

- Effekte: lokale Hyperämisierung durch Kapillarerweiterung, Stoffwechselsteigerung, reflektorische muskuläre Detonisierung.

\subsection{Friktion als Reibung}

- Technik: Schnelles, gleitendes (auch zirkulär fortschreitendes), relativ kräftiges Hin- und Herbewegen der Handballens, des Daumenballens, der Finger- oder Daumenkuppen oder der Fingerknöchel II-V auf der Haut vorwiegend in Längs-, aber auch in Querrichtung zur Längsachse der Extremität über ausgedehnter Muskelfläche (von Gelenk zu Gelenk); ähnlich einer langen 
Streichung unter Druck (Hyperämie des behandelten Gewebes). Die Reibung erfolgt zwischen der Hand des Therapeuten und der darunter liegenden Haut.

- Effekte: es werden Unverschieblichkeiten im Gleitgewebe der Muskeln gegeneinander, der Sehnen und Sehnenscheiden, des Band-Kapsel-Apparats und von Operationsnarben (sog. NarbenMassage) gelöst.

\subsection{Friktion als Zirkelung}

- Technik: Statischer Handgriff mit kreisförmiger, kleinflächiger und örtlich umschriebener, spiralig in die Tiefe des Gewebes ausgeübter Reibung (etwa 2-3/Sekunde). Durchführung mit einer oder mehreren Fingerspitzen, der Daumenkuppe oder dem Handballen (keine fortschreitende Bewegung; die Hand des Therapeuten bleibt an Ort und Stelle und bewegt sich nicht fort).

- Effekte: Permeabilitätssteigerung der Gefäße (evtl. sogar Hämatombildung), Steigerung der Wachreaktion.

\subsection{Klopfen „Tapotement“ (Perkussion)}

- Technik: Alternierende Handgriffe auf einen bestimmten Muskel mit harter Technik, durchgeführt mit den Kuppen der Langfinger, den Fingerknöcheln, der Faust, auch der Hohlhand (Klatschung) oder der ulnaren Handkante mit geschlossenen oder gespreizten Fingern (Hackung). Ausführung federnd im gleichen Rhythmus; gleichzeitige oder wechselseitige Zwei- oder Einhandtechnik; meist zum Abschluss einer Behandlung eingesetzt.

- Effekte: leichte Hyperämie; evtl. leichte muskuläre Tonuserhöhung bei stärkerer Intensität; meist jedoch vorwiegend psychologisch ohne wesentlichen realen therapeutischen Wert.

\subsection{Vibrationen „Erschütterungen“}

- Technik: Feine, niederfrequente, auf die Muskulatur übertragene Schüttel- bzw. Zitterimpulse der flach aufgesetzten Hand; elastisch federnde weiche Schläge aus dem Handgelenk, wobei 3-4 Fingerkuppen nur sehr locker auf dem behandelten Hautbezirk aufliegen; der palpatorische Kontakt zum Gewebe geht nicht verloren.

- Effekte: Tonussenkung bei verspannter Muskulatur (z.B. im Schulter-Nacken- oder lumbalen Bereich), krampflösend und entspannend, sekretmobilisierende Atemtherapie (verbesserte Expektoration), mild sedierend, nur sehr geringe Hauthyperämisierung. 


\subsection{Dehnung als Hautverschiebung}

- Technik: Hier werden die Haut und die Unterhautbindegewebe von der Muskelfaszie mittels verschiedener Formen der Hautverschiebung (rollende, kreisende oder parallele Verschiebung) abgehoben, um sie gegeneinander verschiebbar zu machen, besonders wenn starke Verklebungen bestehen.

- Effekte: Aufbrechen von Verklebungen und Dehnungen der kontraktilen Fasern des Muskels, die zum Ansprechen der Sehnenspindeln führen.

\subsubsection{Dosierung und Behandlungsdauer}

Grundregel: So stark wie nötig, um den gewünschten Behandlungserfolg zu erzielen; so schwach wie möglich, um lokale Unverträglichkeitsreaktionen zu vermeiden. Kurze, dafür häufigere Einzelanwendungen sind therapeutisch effizienter als eine lang andauernde Behandlungsmaßnahme.

Die Reizdosis einer Massage ist von folgenden Faktoren abhängig:

- Größe der Behandlungsfläche

- Dauer der Einzeltherapie: für Rückenmassage 20-30 min, Ganzmassage 40-50 min, Teilmassage einer Körperregion (Arm, Bein) 12-15 min, im Bereich kleiner Gelenke 8-10 min, im jüngeren Alter länger, bei älteren Patienten kürzer dauernde Anwendungen

- Häufigkeit der Wiederholung: In akuten Fällen einmal täglich, bei chronischen Beschwerdebildern 1- bis 3- malige Anwendungen/Woche

- Art des applizierten Handgriffes.

Reizintensität des applizierten Handgriffes: ein starker Druck und auch rasch ausgeführte Streichungen erzeugen eher einen stimulierenden Effekt; ein schwacher Druck bzw. langsam durchgeführte Bewegungen wirken dagegen beruhigend. 


\subsection{Manuelle Lymphdrainage (MLD)}

Die folgenden Aussagen stützen sich auf Bringezu und Schreiner (2001).

\subsubsection{Historische Entwicklung der manuellen Lymphdrainage}

In den 20er und 30er Jahren des 20. Jahrhunderts entwickelte der dänische Biologe Dr. phil. E. Vodder die von ihm so genannte "Lymphdrainage-Massage“, die er auf die besonderen Gegebenheiten des Lymphgefäßsystems ausrichtete.

\subsubsection{Definition}

Lymphdrainage ist eine periphere entstauende manuelle Therapie durch weiche gewebeschonende Massagegriffe überwiegend an der Körperüberfläche; in der letzten Zeit wird sie auch durch apparative Maßnahmen ergänzt ( apparative Lymphdrainage ).

\subsubsection{Ziele der Lymphdrainage}

- Verbesserung der Transportkapazität der Lymphgefäße,

- Periphere Entstauung der Körperregion, damit Schmerzreduktion,

- Verbesserung der Eigenmotorik der Wandmuskulatur der Lymphgefäße,

- Neubildung von Lymphgefäßen zur langfristigen Stabilisierung des peripheren Blutumlaufes,

- Vagotonisierung

\subsubsection{Technik/ Durchführung}

Die Behandlung orientiert sich entlang des anatomischen Verlaufes von Lymphgefäßen in Abflussrichtung. Der Massagedruck ist mit 30-40 mmHg relativ schwach. Ohne aktive Daumen führen die Langfinger des Therapeuten auf ödematösem Gewebe großflächige, gleich- oder gegensinnig kreisende Bewegungen mit abwechselnd stufenlos ansteigendem und dann wieder abfallendem Druck aus.

Bewegungsabfolge: 10/Minute; der Druck sollte lokal mehrere Sekunden aufrechterhalten werden; 6-8 Wiederholungen hintereinander, bevor dann in das Nachbargebiet übergewechselt wird. Die Behandlung beginnt stammnah, um zunächst die zentraleren Lymphbahnen für den Abtransport des peripheren Lymphstaus freizumachen. Sie wird dann schrittweise nach distal gehend fortgesetzt. Die 
Behandlungsrichtung der Drainagegriffe erfolgt von proximal nach distal (im Gegensatz zur Ausstreichung).

Regionen, die nicht berührt oder bewegt werden sollen ( z.B. frische Wunden ), werden von der Behandlung ausgespart.

\subsubsection{Effekte}

- Zunächst Anregung der Lymphgefäße-Vasomotorik im intakten Abflussgebiet,

- Nachfolgende Fibroselockerung im Ödemrandgebiet mit Beseitigung der Abflussbarriere,

- Abschließender Abtransport des Lymphödems aus dem Stauungsgebiet selbst.

\subsubsection{Griffarten}

- Basisgriffe nach Dr. Vodder:

- $\quad$ Stehende Kreise (Einsatz der großflächigen Hand)

- $\quad$ Drehgriff (die Langfinger umkreisen den fest aufliegenden Daumen)

- $\quad$ Schöpfgriff (Daumen und Langfinger kreisen in entgegengesetzter Richtung)

- Quergriff (beidhändig; die distale Therapeutenhand fixiert, proximale Hand mit Durchführung stehender Kreise)

- Ödemgriffe nach Dr. Asdonk:

- Ultrafiltrat-Verdrängungsgriff (lokaler, langsam zunehmender axialer Druck in die Tiefe durch die flache Hand; 20-30 Sekunden)

- Ödemverschiebegriff (beidhändig oder mit beiden Daumen langdauernd und druckstark nach proximal).

\subsubsection{Indikationen}

- Bei Ödemen:

- Sehr selten primäre Fehlanlage (Hypo- oder Aplasie) des Lymphsystems, Elephantiasis

- Meist sekundäre Ödeme z.B.:

- Posttraumatisch (Kontusion, Distorsion mit Hämatom),

- Postoperativ, z.B. nach einer Osteosynthese oder Implantation einer Hüft- oder Knieendoprothese,

○ Nach einer Verbrennung,

○ Nach Immobilisation, Lähmung, 
○ Beim Morbus Sudeck Stadium I-II,

○ Phlebödem, Ulcus cruris, Varikose,

○ Lipödem,

- Nach abgelaufenem Erysipel, nach Lymphangitis,

○ Nach operativer peripherer Tumor- und Lymphknotenausräumung oder radikaler Bestrahlung (Arme nach Mammaoperation, Beine nach Operation im Bereich des kleinen Beckens).

- Bei schwellungsunabhängigen Indikationen

- Akute rheumatische Entzündung

○ Kopfschmerzen

- Relative Indikationen

○ Entzündliches Ödem (proliferatives Stadium),

- Chronische Erkrankungen des rheumatischen Formenkreises,

- Kardial bedingtes peripheres Ödem,

○ Orthostatisches Ödem, Schwangerschaftsödem.

\subsubsection{Kontraindikationen}

- Frische Thrombosen, da Gefahr der Lungenembolie (Latenz 4-6 Wochen),

- Akute bakterielle Entzündung ( z.B.Erysipel) (Gefahr der Keimstreuung),

- Akute Thrombophlebitis, tiefe Beinvenenthrombose,

- Akute Ekzeme oder Hautveränderung unklarer Genese im Behandlungsgebiet.

- Akute Herzinsuffizienz oder dekompensierte Herzinsuffizienz, Lungenödem, Asthma bronchiale (Vagotonisierung), lokales Tumorrezidiv,

- Lokale Einschränkungen: Carotis-Sinus-Syndrom, Hyperthyreose, Herzrhythmusstörung

- keine Bauch-/Bauchtiefbehandlung in der Schwangerschaft, bei entzündlichen Darmerkrankungen, Aneurysmen, Strahlenschäden.

\subsubsection{Dosierung und Behandlungsdauer}

Je nach klinischer Situation 1-5-mal/ Woche. Die Behandlung dauert je nach Indikation zwischen 20 und 60 Minuten. 


\subsection{Zielsetzung}

Untersuchung an den zwei Endpunkten (primärer Endpunkt vier Wochen nach der Behandlung, sekundärer Endpunkt direkt nach Ende der Behandlung):

a) ob die Anzahl der Migräneattacken, Migränetage und Kopfschmerztage unter der Behandlung mit Lymphdrainage oder klassischer Massage abnimmt (definiert als Abnahme der Attacken pro Monat um mindestens $50 \%$ gegenüber einem Baseline-Monat, sogen. Responder, und durch Differenz im Vergleich zu Baseline),

b) ob die Schmerzintensität und die Anzahl der eingenommenen Akutmedikamente abnehmen,

c) ob depressive Symptome abnehmen,

d) ob die Lebensqualität zunimmt und

e) ob sich die Schlafqualität verbessert. 


\section{Material und Methoden}

\subsection{Studiendesign}

Es handelt sich um eine randomisierte, kontrollierte Parallelgruppenstudie, die im Zeitraum September 2007 bis November 2008 im Institut für Physikalische und Rehabilitative Medizin und im Institut für Klinische Neurophysiologie des Klinikums Bremen-Ost in Bremen zur Untersuchung der Wirksamkeit der manuellen Lymphdrainage (MLD) und klassischen Massage (kM) zur Prophylaxe der Migräne mit und ohne Aura durchgeführt worden ist. Die Studie gliedert sich in drei Blöcke (A, B und $C)$ :

Block A - 4 Wochen Baseline Untersuchung zwischen T0 (Zeitpunkt zur Aufnahme) und T1 (Zeitpunkt 4 Wochen nach Aufnahme)

Block B - 8 Wochen Behandlung (1x/Woche 30 Minuten MLD (Gruppe 1, G1) oder kM (Gruppe 2, G2)) oder Wartezeit (Kontrollgruppe, G3) zwischen T1 und T3 (Zeitpunkt 12 Wochen nach Aufnahme).

Block C - 4 Wochen Nachbeobachtung zwischen T3 und T4 (Zeitpunkt des Abschlusses der Studie für beide Interventionsgruppen G1 und G2 4 Wochen nach Behandlungsende). Zeitpunkt T4 gilt auch als Baseline für die Kontrollgruppe (G3), so dass sich danach noch ein Behandlungsblock über 8 Wochen (zwischen T4 und T6) und ein Nachbeobachtungsblock über 4 Wochen (zwischen T6 und T7) anschloss (Studiendesign s. Abbildungen1 und 2).

\begin{tabular}{|c|c|c|c|c|c|c|c|c|c|c|c|c|c|c|c|c|}
\hline Zeitpunkte & \multicolumn{4}{|c|}{ TO } & \multicolumn{4}{|c|}{ T1 } & \multicolumn{4}{|l|}{ T2 } & \multicolumn{4}{|l|}{ T3 } \\
\hline Woche & 1 & 2 & 3 & 4 & 5 & 6 & 7 & 8 & 9 & 10 & 11 & 12 & 13 & 14 & 15 & 16 \\
\hline $\begin{array}{l}\text { Phase } \\
\text { (G1und G2) }\end{array}$ & \multicolumn{4}{|c|}{ Baseline } & \multicolumn{8}{|c|}{ Intervention bzw. Behandlung } & \multicolumn{4}{|c|}{ Nachbeobachtung } \\
\hline Phase (G3) & \multicolumn{12}{|c|}{ Wartezeit (Kontrollgruppe) } & \multicolumn{4}{|c|}{$\begin{array}{l}\text { Wartezeit } \quad \text { bzw. } \\
\text { Baseline }\end{array}$} \\
\hline
\end{tabular}

\begin{tabular}{|c|c|c|c|c|c|c|c|c|c|c|c|c|c|c|c|c|}
\hline Zeitpunkte & \multicolumn{4}{|l|}{ T3 } & \multicolumn{4}{|l|}{ T4 } & \multicolumn{4}{|l|}{ T5 } & \multicolumn{4}{|l|}{ T6 } \\
\hline Woche & 13 & 14 & 15 & 16 & 17 & 18 & 19 & 20 & 21 & 22 & 23 & 24 & 25 & 26 & 27 & 28 \\
\hline Phase & \multicolumn{4}{|c|}{ Baseline } & \multicolumn{8}{|c|}{ Intervention bzw. Behandlung } & \multicolumn{4}{|c|}{ Nachbeobachtung } \\
\hline
\end{tabular}




\subsection{Patienten}

Es wurden Patienten $\geq 18$ Jahre mit gesicherter Migräne mit oder ohne Aura nach den Kriterien der International Headache Society mit zwei oder mehr Attacken pro Monat zwischen September 2007 und November 2008 eingeschlossen. Die Patienten wurden 1) über eine neurologische Praxis eines niedergelassenen Neurologen mit Schwerpunkt Kopfschmerzbehandlung (31 Patienten, 41,9\%) und 2) über Aushänge im Klinikum Bremen-Ost und Anzeigen in Lokalzeitungen (43 Patienten, 58,1\%) rekrutiert.

Alle an der Studie teilnehmenden Patienten wurden über die Ziele sowie die Durchführung der Studie aufgeklärt und haben ihr schriftliches Einverständnis anhand einer Einverständniserklärung gegeben (siehe Anhang 1 und 2). Zudem wurde die Studie von der Ethikkommission der Ärztekammer Bremen geprüft und unter der Prüfnummer 167 (im Jahr 2007) genehmigt.

\subsection{Studienablauf}

Zum Aufnahmezeitpunkt „T0“ wurden 74 Patienten (66 Frauen und 8 Männer) nach Überprüfung der Ein- und Ausschlusskriterien (Tabelle 10) eingeschlossen. Bei Aufnahme wurden ein Gespräch mit jedem Patienten sowie eine klinische Untersuchung durchgeführt. In dem Gespräch wurden medizinische und soziale Hintergründe exploriert, die Diagnose nach den Kriterien der International Headache Society 2004 gesichert, die Ein- und Ausschlusskriterien geprüft und die Patienten bei Eignung zur Teilnahme an der Studie über Studienablauf, Ziel, Zweck und über die möglichen Risiken der Untersuchung informiert (s. Anhang 1 Patienteninformation).

Die Patienteneinverständniserklärung wurde nach diesem Gespräch von den Patienten unterzeichnet (s. Anhang 2).

Die klinische Untersuchung umfasste die Erfragung von Körpergröße, Körpergewicht sowie die Messung des Blutdrucks im Sitzen und der Pulsfrequenz. Neben einer neurologischen Untersuchung erfolgte eine orientierende internistische Untersuchung. Klinische und demographische Daten wurden in einem Standarderhebungsbogen erfasst (s. Anhang 3).

Zunächst wurden die Patienten gebeten, über vier Wochen ein Kopfschmerztagebuch zu führen und sich anschließend wieder vorzustellen zum zweiten Zeitpunkt „T1“. Als Einschlusskriterium mussten innerhalb der vergangenen vier Wochen zwei oder mehr Migräneattacken aufgetreten und im Tagebuch verzeichnet sein.

Nach endgültiger Aufnahme in die Studie wurden die Patienten in drei Gruppen randomisiert (siehe Kapitel 3.5): 
Gruppe 1 (G1) mit 25 Patienten zur Behandlung mit manueller Lymphdrainage (MLD)

Gruppe 2 (G2) mit 24 Patienten zur Behandlung mit klassischer Massage (kM)

Gruppe 3 (G3) mit 25 Patienten als Kontrollgruppe (Wartegruppe).

Die Gruppe 3 wurde in zwei Gruppen (G3a: 12 Patienten / G3b: 13 Patienten) zur anschließenden gleichen Behandlung wie die beiden ersten Gruppen randomisiert (G3a $=M L D, G 3 b=k M$ ).

\begin{tabular}{|l|l|}
\hline Tabelle 10: Einschluss- und Ausschlusskriterien & Ausschlusskriterien \\
\hline Einschlusskriterien & - weniger als 2 Attacken in der Baseline-Phase \\
\hline - Alter $\geq 18$ Jahre & - anderer idiopathischer oder sekundärer \\
- gesicherte Migräne mit oder ohne Aura & Kopfschmerz (Ausnahme: Kopfschmerz vom \\
- 2 oder mehr Migräneattacken pro 4 Wochen (mit & Spannungstyp) \\
einer Dauer von mindestens 2 Stunden bei & - relevante neurologische, psychiatrische oder \\
Einnahme von Akutmedikation oder von 4-72 & internistische Erkrankung \\
Stunden ohne Einnahme von Akutmedikation) in & - unregelmäßige Teilnahme an den Interventionen \\
den vergangenen 3 Monaten & - Änderung der medikamentösen Medikation zur \\
- falls eine medikamentöse Migräneprophylaxe & Migräneprophylaxe \\
eingenommen wird, muss diese in den & - Einnahme von Analgetika an mehr als 3 Tagen \\
vergangenen 3 Monaten stabil gewesen sein und & pro 4 Wochen wegen anderer chronischer \\
während der Studiendauer stabil gehalten werden & Schmerzen \\
- Möglichkeit des Führens eines & - Aktuelle Kortisontherapie \\
Kopfschmerztagebuches und des Ausfüllens der & - Medikamentenmissbrauch \\
Fragebögen & - Schwangerschaft oder Stillzeit \\
- Unterschriebene Einverständniserklärung & - Teilnahme an einer anderen Studie in den \\
& vergangenen 3 Monaten \\
& - Kontraindikationen der manuellen \\
& Lymphdrainage oder der klassischen Massage \\
\hline
\end{tabular}

Zu denselben Zeitpunkten „T1, T2, T3, T4“ für die Behandlungsgruppen und „T1, T2, T3, T4, T5, T6, T7“ für die Kontrollgruppe wurden alle aufgenommene Patienten ärztlich gesehen, befragt und füllten unten angegebene Fragebögen aus. Das Kopfschmerztagebuch und die Ausschlusskriterien wurden zu denselben o.g. Zeitpunkten kontrolliert.

Von 74 Patienten zum Aufnahmezeitpunkt (T0) konnten Daten nach 4 Wochen Baseline (T1) von 64 Patienten ( $n=21$ in $G 1, n=21$ in $G 2, n=22$ in G3), nach 4 Wochen Behandlung (T2) von 62 Patienten ( $n=20$ in $G 1, n=20$ in $G 2, n=22$ in G3), nach 8 Wochen Behandlung (T3) von 58 Patienten ( $n=19$ in $G 1$, n=19 in G2, n=20 in G3), und nach 4 Wochen Nachbeobachtung (T4) (Baseline für Kontrollgruppe) von 56 Patienten ( $n=18$ in $\mathrm{G} 1, n=19$ in $\mathrm{G} 2, \mathrm{n}=19$ in G3) analysiert werden (Abbildung 3).

Von 25 Patienten in der Kontrollgruppe „G3“ ( $n=12$ in G3a, n=13 in G3b) zum Aufnahmezeitpunkt (T0) konnten Daten in der Interventionsphase zur Baseline (T4) von 18 Patienten ( $n=7$ in G3a, $n=11$ in G3b), nach 4 Wochen Behandlung (T5), nach 8 Wochen Behandlung (T6) und zum Ende der Nachbeobachtung (T7) von 17 Patienten ( $n=7$ in G3a, $n=10$ in G3b) analysiert werden (s. Abbildung 4). 


\begin{tabular}{|c|c|}
\hline \multicolumn{2}{|c|}{$\begin{array}{l}\text { T0: Zeitpunkt der Aufnahme in die Studie. } \\
\text { Keine Datenerhebung zu diesem Zeitpunkt } \\
\text { n = } 74 \text { Patienten (G1:n=25, G2: n=24, G3: n=25) }\end{array}$} \\
\hline$\downarrow$ & $\begin{array}{l}\text { Drop-out-Gründe }(\mathrm{n}=10) \\
\qquad \begin{aligned} 1 \text { Rücknahme der Einwilligung } \\
9 \text { Ausschlusskriterien } \\
\circ \quad 5 \text { weniger als } 2 \text { Attacken } \\
0 \quad 1 \text { Schwangerschaft } \\
0 \quad 1 \text { zu geringe Möglichkeit, } \\
\text { Fragebögen korrekt } \\
\text { auszufüllen }\end{aligned}\end{array}$ \\
\hline \multicolumn{2}{|c|}{$\begin{array}{l}\text { T1: Zeitpunkt zur Baseline/ } 4 \text { Wochen nach der Aufnahme } \\
\qquad \text { n=64 (21/21/22) } \\
\text { Datenerhebung (Kpsk, Midas, IBK, CES-D, SF-36, PSQI, CGI) }\end{array}$} \\
\hline$\downarrow$ & $\begin{array}{l}\text { Drop-out-Gründe }(\mathrm{n}=2) \\
2 \text { akute Erkrankung (Gastroenteritis, } \\
\text { Grippe verursacht unregelmäßiges } \\
\text { Erscheinen) }\end{array}$ \\
\hline $\begin{array}{l}\text { T2: Zeitpunkt } 8 \text { Wochen nach Aufnahn } \\
\text { n=62 (20/20/2 } \\
\text { Datenerhebung (Kpsk, Midas, IBK, C }\end{array}$ & $\begin{array}{l}\text { ne (nach } 4 \text { Behandlungen) } \\
\text { 22) } \\
\text { ES-D, SF-36, PSQI, CGI) }\end{array}$ \\
\hline$\downarrow$ & 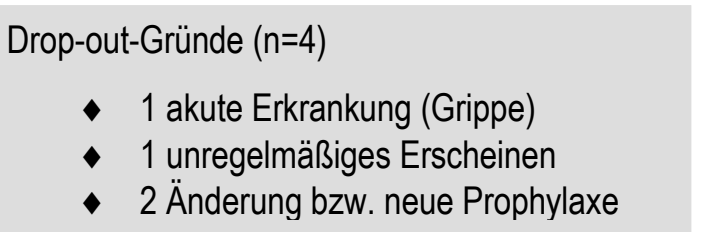 \\
\hline $\begin{array}{r}\text { T3: Zeitpunkt } 12 \text { Wochen nach Aufnah } \\
\text { n=58 (19/19/2 } \\
\text { Datenerhebung (Kpsk, Midas, IBK, C }\end{array}$ & $\begin{array}{l}\text { Ime (nach } 8 \text { Behandlungen) } \\
\text { 20) } \\
\text { ES-D, SF-36, PSQI, CGI) }\end{array}$ \\
\hline$\downarrow$ & $\begin{array}{l}\text { Drop-out-Gründe }(\mathrm{n}=2) \\
\qquad \text { unregelmäßiges Erscheinen }\end{array}$ \\
\hline \multicolumn{2}{|c|}{$\begin{array}{l}\text { T4: Zeitpunkt } 16 \text { Wochen nach Aufnahme (nach } 4 \text { Wochen Nachbeobachtung) } \\
\qquad n=56(18 / 19 / 19)\end{array}$} \\
\hline $\begin{array}{l}\text { Abbildung 3: Zeitlicher Ablauf der Datenerhebung (Hauptte } \\
\text { n (G1/G2/G3) = Zahl der Patienten (G1=Lymphdrainage/G2=kl } \\
\text { Kpsk: Kopfschmerzkalender, Midas: Migraine disability assess } \\
\text { Kopfschmerzen, CES-D: Center for Epidemiologic Studies Dep } \\
\text { PSQI: Pittsburgh Sleep Quality Index, CGI: Clinical Global Impr }\end{array}$ & $\begin{array}{l}\text { lil dieser Studie) } \\
\text { assische Massage/G3=Kontrollgruppe) } \\
\text { ment, IBK: Inventar zur Beeinträchtigung durch } \\
\text { ression Scale, SF-36: Lebensqualitätsfragebogen, } \\
\text { ression. }\end{array}$ \\
\hline
\end{tabular}


T4: Zeitpunkt 16 Wochen nach Aufnahme

gilt als Baseline für Kontrollgruppezur Intervention

$$
n=18 \text { (G3a: n=7, G3b: n=11) }
$$

Datenerhebung (Kpsk, Midas, IBK, CES-D, SF-36, PSQI, CGI)

Drop-out-Gründe ( $n=1)$

- 1 akute Erkrankung

(Grippe)

T5: Zeitpunkt 20 Wochen nach Aufnahme

nach 4 Behandlungen

$\mathrm{n}=17(7 / 10)$

Datenerhebung (Kpsk, Midas, IBK, CES-D, SF-36, PSQI, CGI)

T6: Zeitpunkt 24 Wochen nach Aufnahme

nach 8 Behandlungen

$\mathrm{n}=17(7 / 10)$

Datenerhebung (Kpsk, Midas, IBK, CES-D, SF-36, PSQI, CGI)

T7: Zeitpunkt 28 Wochen nach Aufnahme

4 Wochen Nachbeobachtung

$$
\mathrm{n}=17(7 / 10)
$$

Datenerhebung (Kpsk, Midas, IBK, CES-D, SF-36, PSQI, CGI)

Abbildung 4: Zeitlicher Ablauf der Datenerhebung bei der Kontrollgruppe während der Intervention $\mathrm{n}$ (G3a/G3b): Zahl der Patienten in der Kontrollgruppe (G3a: Lymphdrainage / G3b: klassische Massage) Kpsk: Kopfschmerzkalender, Midas: Migraine disability assessment, IBK: Inventar zur Beeinträchtigung durch Kopfschmerzen, CES-D: Center for Epidemiologic Studies Depression Scale, SF-36:

Lebensqualitätsfragebogen, PSQI: Pittsburgh Sleep Quality Index, CGI: Clinical Global Impression. 


\subsection{Messparameter}

Hauptparameter in dieser Studie sind die Zahl der Migräneattacken, Migränetage, Kopfschmerztage und die Zahl der eingenommenen Medikamente während der Attacken. Es werden die Schmerzintensität und die Begleitsymptome wie Übelkeit, Erbrechen, Photophobie und Phonophobie sowie psychometrische Parameter (akute Beeinträchtigung durch Kopfschmerzen (Gesamtscore) gemessen durch das Inventar für Beeinträchtigung durch Kopfschmerzen (IBK) (s. 3.4.2.1), Einschränkungen der Leistungsfähigkeit gemessen durch migraine disability assessment (Midas) (s.3.4.2.2), Intensität der depressiven Symptome gemessen durch Center for Epidemiologic Studies Depression Scale (CES-D) (s.3.4.2.3), Lebensqualität gemessen in acht Subskalen und zwei Summenskalen des SF-36 (s.3.4.2.4), Schlafqualität gemessen durch Pittsburgh Sleep Quality Index (PSQI) (s.3.4.2.5) und objektive Symptomschwere gemessen durch Clinical Global Impression (CGI) (s.3.4.2.6)) durch das Kopfschmerztagebuch und die entsprechenden Fragebögen gemessen. Diese Parameter werden hauptsächlich zu zwei Endpunkten verglichen. Beim primären Endpunkt werden die Responderrate und die Parameter am Ende der Nachbeobachtung (T4) mit der Baseline (T1) verglichen. Beim sekundären Endpunkt werden die Responderrate sowie die Parameter am Ende der Behandlung (T3) mit der Baseline (T1) verglichen.

\subsubsection{Kopfschmerztagebuch (Kopfschmerzkalender)}

Über den gesamten Untersuchungszeitraum (inkl. Baseline und Nachbeobachtungsphase) wurde das Kopfschmerztagebuch der DMKG (s. Anhang 4) von den Patienten selbst ausgefüllt und zu jeder Untersuchung mitgebracht. Das Baseline-Tagebuch wurde für das Einschlusskriterium von 2 oder mehr Migräne-Attacken pro 4 Wochen herangezogen. Die Zahl der Migräneattacken wurde gezählt in Abschnitten von je 4 Wochen. Als eine Migräneattacke gelten alle Abschnitte mit Kopfschmerzen, die die Kriterien der IHS für eine Migräne erfüllten und in denen der Kopfschmerz weniger als 24 Stunden (oder nur durch Schlaf) unterbrochen war. Hier wurde von den Patienten die Kopfschmerzintensität in einer Skala von 1 bis 3 (1: leichter Schmerz, 2: mittelstarker Schmerz, 3: starker Schmerz) eingetragen. Ebenfalls wurden die maximale Zahl der eingenommenen Medikamente sowie die Begleitsymptome während der Migräneattacken dort eingetragen.

\subsubsection{Fragebögen}

Folgende Fragebögen wurden von den Patienten ab der Baseline zu jedem o. g. Zeitpunkt ausgefüllt (s. Anhang 5 bis 10): 
- Inventar zur Beeinträchtigung durch Kopfschmerzen, IBK (Bauer et al. 1999)

- Migraine disability assessment, Midas-Fragebogen (Stewart et al. 1999)

- Center for Epidemiologic Studies Depression Scale, CES-D (Andresen et al. 1994)

- Lebensqualitätsfragebogen, SF-36 (Ware et al. 1993)

- Pittsburgh Sleep Quality Index, PSQI (Buysse et al. 1989).

Eine Fremdeinschätzung des Schweregrades der Migräne wurde bei jeder Vorstellung anhand der Clinical Global Impression Scale (CGI) durch den betreuenden Arzt abgegeben.

\subsubsection{Inventar zur Beeinträchtigung durch Kopfschmerzen}

Das Inventar zur Beeinträchtigung durch Kopfschmerzen (IBK) ist das erste kopfschmerzspezifische Instrument zur Erfassung der akuten Beeinträchtigung der Lebensqualität für den deutschsprachigen Raum und entspricht dem Headache Disability Inventory (HDI) für den englischsprachigen Raum (Jacobson et al. 1994). Das IBK (Anhang 5) besteht aus 25 Items, die in zwei Subskalen unterteilt sind. Die Subskala „Funktion“ misst Einschränkungen normaler Aktivitäten des täglichen Lebens und enthält 12 Items $(2,4,7,13,15,16,17,18,19,21,24,25)$. Die Subskala „Emotionen“ erfasst die durch Kopfschmerzen verursachten Befindlichkeitsstörungen und besteht aus 13 Items $(1,3,5,6,8,9,10,11$, 12, 14, 20, 22, 23). Der Gesamtscore kann als Kontrolle des Therapieerfolges dienen und wurde in dieser Studie verwendet.

Die einzelnen Items können durch „ja“, „manchmal“ oder "nein“ beantwortet werden. Für ein „ja“ werden bei der Bewertung 4 Punkte, für "manchmal“ 2 und für „nein“ 0 Punkte vergeben, also kann die Punktzahl für „Funktion“ 0 bis 48 Punkte und die Punktzahl für „Emotionen“ 0 bis 52 Punkte betragen. Je größer die Summe in den Subgruppen wird, desto stärker kann die Beeinträchtigung durch Kopfschmerzen angenommen werden. Nach „The Henry Ford Hospital Headache Disability Inventory“ muss im Gesamtscore im Verlauf ein Gewinn von mindestens 29 Punkten erreicht werden, um mit einer relevanten Verbesserung zu rechnen (Jacobson et al 1994).

\subsubsection{Migräne disability assessment, Midas-Fragebogen}

Der Migraine Disability Assessment Questionnaire und der sich daraus ergebende Migraine Disability Assessment Score (Midas) wurde entwickelt, um migränebedingte Einschränkungen der Leistungsfähigkeit messen zu können und dadurch unter anderem die Arzt-Patienten-Kommunikation zu verbessern.

Es handelt sich um einen Fragebogen mit fünf Fragen, den die Patienten selbstständig ausfüllen können. Er summiert die Anzahl der Tage, an denen die Patienten migränebedingt vollständig oder 
mindestens zur Hälfte in ihrer Leistungsfähigkeit beeinträchtigt gewesen sind, dies über einen Zeitraum von drei Monaten. Dabei unterscheidet der Fragebogen zwischen dem Arbeitsplatz bzw. der Schule und zu Hause. Bei der Auswertung ergibt sich aus der Summe der durch „Migräne verlorenen Tage" der MIDA-Score, welcher gemäß der Tabelle im Anhang in Grad I (minimale oder infrequente Beeinträchtigung) bis Grad IV (schwere Beeinträchtigung) unterteilt wird. Durch die Gradeinteilung wird die Behandlungsbedürftigkeit sowie die erforderliche Vorgehensweise ersichtlich (sieh Anhang 6) (Stewart et al.1999). In dieser Studie werden die gesamten migränebedingt-verlorenen Tage bzw. der Gesamtscore für den Midas verwendet (ohne Gradierung).

\subsubsection{Center for Epidemiologic Studies Depression Scale, CES-D}

Die Center for Epidemiologic Studies Depression Scale wurde von Ben Locke und Peter Putnam ursprünglich entwickelt, um die Intensität der depressiven Symptome von Erwachsenen zu bewerten (Randolf 1977). In deutscher Fassung wurde sie als Allgemeine Depressionsskala (ADS) benannt (Hautzinger und Bailer 1993). Die Skala besteht aus 20 Items (16 positiv gepolt, 4 negativ gepolt), die die typischen depressiven Symptome abfragen wie z.B. Verunsicherung, Erschöpfung, Hoffnungslosigkeit, Selbstabwertung, Niedergeschlagenheit, Einsamkeit, Traurigkeit, Antriebslosigkeit, Weinen, Rückzug, Angst u.a.. Der Bezugszeitraum ist die vergangene Woche. Der Patient hat die Möglichkeit, die Häufigkeit und Dauer der Symptome anhand einer vierstufigen Skala anzugeben. Die Kategorien sind: 0 Punkte: „kaum oder überhaupt nicht“ (weniger als 1 Tag); 1 Punkt: „manchmal“ (1 bis 2 Tage); 2 Punkte: „ofters“ (3 bis 4 Tage); 3 Punkte: „meistens“ (5 bis 7 Tage). Die Auswertung erfolgt durch Addition der einzelnen Punkte. Bei den negativ gepolten Items (4, 8, 12 und 16) ist der umgekehrte Wert zu addieren $(0=>3 ; 1=>2 ; 2=>1 ; 3=>0)$. Die Befunde gelten als auffällig, wenn ein CES-D-Wert von 16 und mehr Punkten erreicht wird (Andresen et al. 1994).

\subsubsection{Lebensqualitätsfragebogen, SF-36}

Zur Erfassung der gesundheitsbezogenen Lebensqualität existieren viele Messinstrumente, die sowohl psychometrisch geprüft als auch international verfügbar und normiert sind. Eines dieser Messinstrumente ist der ursprünglich amerikanische SF-36 Health Survey, der mit 36 Fragen acht Dimensionen der subjektiven Gesundheit und zwei Summenscores krankheitsunabhängig erfasst (Ware et al. 1993, deutsche Fassung: Bullinger und Kirchberger 1998) (Anhang 8). In Tabelle 11 sind diese Dimensionen zusammengefasst. Die Auswertung des SF-36 wurde nach dem Auswertungskapitel der Bullinger und Kirchberger Handweisung durchgeführt. Hierzu wurden die Roh-Skalenwerte in 0-100 Skalenwerte transformiert (Bullinger und Kirchberger 1998). 


\begin{tabular}{|l|l|l|l|}
\hline \multicolumn{3}{|l|}{$\begin{array}{l}\text { Tabelle 11: Gesundheitskonzepte, Itemzahl und Anzahl der Stufen sowie Inhalt der acht SF-36 Skalen und } \\
\text { des Items zur Veränderung des Gesundheitszustandes (nach Bullinger und Kirchberger 1998, S. 12) }\end{array}$} \\
\hline Konzepte & Itemzahl & $\begin{array}{l}\text { Anzahl der } \\
\text { Stufen }\end{array}$ & \\
\hline $\begin{array}{l}\text { Körperliche } \\
\text { Funktionsfähigkeit }\end{array}$ & 10 & 21 & $\begin{array}{l}\text { Ausmaß, in dem der Gesundheitszustand körperliche } \\
\text { Aktivitäten wie Selbstversorgung, Gehen, Treppen } \\
\text { steigen, bücken, heben und mittelschwere oder } \\
\text { anstrengende Tätigkeiten beeinträchtigt }\end{array}$ \\
\hline Körperliche Schmerzen & 2 & 11 & $\begin{array}{l}\text { Ausmaß an Schmerzen und Einfluss der Schmerzen } \\
\text { auf die normale Arbeit, sowohl im Haus als auch } \\
\text { außerhalb des Hauses }\end{array}$ \\
\hline $\begin{array}{l}\text { Allgemeine Gesundheits- } \\
\text { wahrnehmung }\end{array}$ & 5 & 21 & $\begin{array}{l}\text { Persönliche Beurteilung der Gesundheit, einschließlich } \\
\text { aktueller Gesundheitszustand, zukünftige Erwartung } \\
\text { und Widerstandsfähigkeit gegenüber Erkrankung }\end{array}$ \\
\hline Vitalität & 4 & 21 & $\begin{array}{l}\text { Sich Energie geladen und voller Schwung fühlen } \\
\text { versus müde und erschöpft fühlen }\end{array}$ \\
\hline Soziale Funktionsfähigkeit & 2 & 9 & $\begin{array}{l}\text { Ausmaß, in dem die körperliche Gesundheit oder } \\
\text { emotionale Probleme normale soziale Aktivitäten } \\
\text { beeinträchtigen }\end{array}$ \\
\hline Emotionale Rollenfunktion & 3 & 4 & $\begin{array}{l}\text { Ausmaß, in dem die emotionalen Probleme die Arbeit } \\
\text { oder andere tägliche Tätigkeiten beeinträchtigen: u.a. } \\
\text { weniger Zeit aufbringen, weniger schaffen und nicht } \\
\text { sorgfältig wie üblich arbeiten }\end{array}$ \\
\hline Psychisches Wohlbefinden & 5 & 26 & $\begin{array}{l}\text { Allgemeine psychische Gesundheit einschließlich } \\
\text { Depression, Angst, emotionale und } \\
\text { verhaltensbezogene Kontrolle, allgemeine positive } \\
\text { Gestimmtheit }\end{array}$ \\
\hline $\begin{array}{l}\text { Veränderung der } \\
\text { Gesundheit }\end{array}$ & 1 & 5 & $\begin{array}{l}\text { Beurteilung des aktuellen Gesundheitszustandes im } \\
\text { Vergleich zum vergangenen Jahr }\end{array}$ \\
\hline
\end{tabular}

\subsubsection{Pittsburgh Sleep Quality Index, PSQI}

Der PSQI erfragt retrospektiv für einen Zeitraum von vier Wochen die Häufigkeit schlafstörender Ereignisse, die Einschätzung der Schlafqualität, die gewöhnlichen Schlafzeiten, Einschlaflatenz und Schlafdauer, die Einnahme von Schlafmedikation sowie die Tagesmüdigkeit. Insgesamt werden 18 Items zur quantitativen Auswertung herangezogen und sieben Komponenten (subjektive Schlafqualität, Schlaflatenz, Schlafdauer, Schlafeffizienz, Schlafstörungen, Schlafmittelkonsum, Tagesschläfrigkeit) zugeordnet, die jeweils einen Wertebereich von 0 bis 3 annehmen können. Der Gesamtscore ergibt sich aus der Summation der Komponentenscores und kann von 0 bis 21 variieren, wobei eine höhere Ausprägung einer verringerten Schlafqualität entspricht. Es besteht ein empirisch bestimmter Cut-offWert von 5, der eine Einteilung in "gute" und "schlechte" Schläfer erlaubt, jedoch keine differenzialdiagnostische Unterscheidung verschiedener Schlafstörungen (Buysse et al. 1989) (Anhang 9).

\subsubsection{Clinical Global Impression Scale, CGI}


Hier wird die Symptomschwere durch den betreuenden Arzt in einer Skala von sieben Punkten angegeben. Eine 7-Punkte-Skala verlangt, dass der Kliniker den Schweregrad der Erkrankung des Patienten zur Zeit der Bewertung hinsichtlich der vorigen Erfahrung des Klinikers mit Patienten abschätzt, die dieselbe Diagnose haben. Abhängig von der klinischen Gesamterfahrung wird ein Patient nach dem Schweregrad der Erkrankung zur Zeit der Schätzung von $1=$ normal (überhaupt nicht krank), $2=$ grenzwertig krank, $3=$ leicht krank, $4=$ mäßig krank, $5=$ deutlich krank, $6=$ schwer $\operatorname{krank}$ oder $7=$ sehr schwer krank (unter den am schwersten betroffenen) bewertet (s. Anhang 10) (Guy 1976).

\subsection{Randomisierung}

Um eine bessere Balanciertheit der einzelnen Gruppen gewährleisten zu können, wurde eine BlockRandomisierung durchgeführt, d. h. die einzuschließenden (unbekannt nummerierten) Patienten wurden vor Beginn der Studie in 13 Blöcke aufgeteilt. In jedem Block ist das Verhältnis der Behandlungsgruppen zur Kontrollgruppe als 2:1 oder 4:2 definiert. Jeder Block erhielt sechs Patienten bzw. Nummern: 1 für Lymphdrainage (G1), 2 für klassische Massage (G2), 3 für Kontrollgruppe mit später Lymphdrainage (G3a) und 4 für Kontrollgruppe mit später klassischer Massage (G3b). Die Zuweisungsfestlegungen (1, 1, 2, 2, 3, 4) wurden in jedem Block zufällig mit Hilfe einer Excel-Tabelle bestimmt und innerhalb der Randomisierungsliste zufällig aneinander gereiht (Tabelle12 als Beispiel für das Randomisierungsprotokoll).

\begin{tabular}{|l|l|l|l|l|l|l|l|l|l|l|}
\hline \multicolumn{2}{|l|}{ Tabelle 12: Randomisierungsprotokoll (Patienten von 1 bis 30 als Beispiel) } \\
\hline Pat.Nr. & Rand.Nr. & Pat.Nr. & Rand.Nr. & Pat.Nr. & Rand.Nr. & Pat.Nr. & Rand.Nr. & Pat.Nr. & Rand.Nr. \\
\hline 1 & 1 & 7 & 2 & 13 & 1 & 19 & 4 & 25 & 3 \\
\hline 2 & 2 & 8 & 1 & 14 & 2 & 20 & 1 & 26 & 4 \\
\hline 3 & 3 & 9 & 2 & 15 & 1 & 21 & 2 & 27 & 1 \\
\hline 4 & 4 & 10 & 3 & 16 & 2 & 22 & 1 & 28 & 2 \\
\hline 5 & 1 & 11 & 4 & 17 & 3 & 23 & 2 & 29 & 1 \\
\hline 6 & 2 & 12 & 1 & 18 & 4 & 24 & 3 & 30 & 2 \\
\hline
\end{tabular}

\subsection{Interventionen}

Während der Interventionsphase wurden die Patienten nach Gruppen einer von zwei Therapieformen zugeteilt. Die Therapie erfolgte einmal in der Woche à 30 Minuten für 8 Wochen nacheinander (mit einem Abstand von 6 bis max. 9 Tagen zwischen jeder Therapieeinheit). Die Interventionen wurden durch eine/n Masseur/in und medizinische/n Bademeister/in des Instituts für Physikalische und Rehabilitative Medizin (PRM) im Klinikum Bremen-Ost und in den Räumen dieses Institutes durchgeführt. 


\subsubsection{Behandlungsprotokoll für Gruppe 1 und Gruppe 3a „Manuelle Lymphdrainage Gesicht“}

- Die Behandlungen erfolgen in Rückenlage auf einer Massagebank

- $\quad$ Der Masseur steht oder sitzt entweder seitlich der Behandlungsliege oder am Kopfende

- Der Patient sollte während der gesamten Behandlung die Augen möglichst geschlossen halten und entspannen

- $\quad$ Die Behandlung beginnt und endet in jeder Region mit einer einleitenden Ausstreichung.

Es wurde bei jedem Patienten der o.g. Gruppen während der Interventionsphase im Hals-, Gesichtsund Nackenbereich die folgende Griffolge eingesetzt (modifiziert nach Bringezu und Schreiner 2001, S. 241-255):

- Halsregion: Basisbehandlung

1. Einleitungseffleurage

2. Jugularis-Terminus: stehende Kreise seitlich am Hals in mehreren Ansätzen bis zur Schlüsselbeingrube

3. Okziput-Terminus: stehende Kreise dorsal am Hals in mehreren Ansätzen bis zur Schlüsselbeingrube

4. Mundboden-Parotis-Grifffolge: stehende Kreise am Kopf-Hals-Übergang bis zur Schlüsselbeingrube

\section{- Behandlung des Gesichts:}

1. Ober-/Unterkiefer:

- Mundboden-Grifffolge vom Kinn bis zum Angulus mandibularis

- $\quad$ Unterkiefer-Grifffolge vom Kinn bis zum Angulus mandibularis

- $\quad$ Oberkiefer-Grifffolge von Mitte Oberlippe bis zum Angulus mandibularis

- Jugularis-Terminus: Ableiten über laterale Jugulariskette zur Einmündungsstelle

2. Wangenbehandlung: „lange Reise“ von der Jochbeinregion bis zur Schlüsselbeingrube

3. Augenbehandlung:

- Tränensackregion

- $\quad$ Behandlung auf dem oberen und unteren knöchernen Augenhöhlenrand (stehende Kreise zwischen Augapfel und Orbitarand)

- Behandlung auf dem Augapfel

- Behandlung auf den Augenbrauen

4. Stirnbehandlung einschließlich Temporalregion

5. Behandlung des behaarten Kopfteils, soweit aus der Rückenlage möglich

6. Seitliches Ableiten über Temporalis-Parotis-Terminus 


\section{- Behandlung des Nackens:}

Die Grifffolge wird in Bauchlage oder im Sitzen ausgeführt. Der Therapeut steht oder sitzt entweder am Kopfende der Liege oder seitlich davon.

1. Jugularis-Terminus

2. Okziput-Terminus: Entlang der Protuberantia occipitalis von medial nach lateral, anschließend auf der dorsalen Halsseite in mehreren Ansätzen bis zur Schlüsselbeingrube

3. Paravertebrale Daumenkreise

4. Hinterhaupt-Behandlung: beginnend an der Linea nuchea in vielen Ansätzen nach kranial bis zum Schädeldach, anschließend entlang der Seitenlinie des Kopfes bis zur Ohrenregion (unter Mitbehandlung der prä- und retroaurikulären Lymphknoten), abschließend über die jugulare Lymphknotenkette bis zur Schlüsselbeingrube

5. Beidhändige stehende Kreise auf den freien Rändern des M. trapezius mit abschließender Terminusbehandlung. 


\subsubsection{Behandlungsprotokoll für Gruppe 2 und Gruppe 3b „Klassische Rückenmassage“}

- Die Behandlungen werden in Bauchlage auf der Massagebank durchgeführt

- Gearbeitet wird immer auf der abgewandten (gegenüberliegenden) Seite, begonnen wird immer mit der rechten Rückenhälfte (herzfern)

- $\quad$ Die Arme liegen neben dem Körper (bessere Entspannung im Schulter-Nackengürtel)

- Das Gesicht ist dem Massierenden zugewandt

- $\quad$ Die Füße werden mit einer Halbrolle unterlagert

- $\quad$ Bei Patienten mit Hohlkreuz wird der Bereich mit einem flachen Kissen unterlagert.

Behandlungsreihenfolge (nach Grundlage der klassischen Rückenmassage):

1. Es erfolgt eine Ausstreichung vom Kreuzbein aus zum Hinterhauptbein - Os occipitale - und dann über den oberen Rand - pars descendens - des M. trapezius beiderseits zum Schultergelenk. Auf dem Rückweg vorbei an den Schulterblättern bis zur Achselhöhle und auf der Außenseite des Rückens zum Lendenbereich. Im Lendenbereich wird die Beckenschaufel umfahren und die Hände werden in Richtung der eigenen Körpermitte zurückgezogen. Hier wird die Gesäßmuskulatur mit kräftigen Streichungen behandelt

2. Diese Ausstreichung wird wahlweise mit beiden Händen, einer Hand oder beschwert mit einer auf der anderen Hand ausgeführt

3. Gleiche Strichführung mit umgeklappter (Plättgriff) Hand, ein- oder beidhändig

4. Es erfolgt dann eine Knetung der Gesäßmuskulatur, aufsteigend am Rücken hoch bis zum Hinterhauptbein. Hier wird die Muskulatur mit sanftem Druck gedehnt

5. Mit gleicher Strichführung erfolgt die Friktion im gleichen Muster wie die Ausstreichung

6. Dann erfolgt ein Seitenwechsel

7. Zwischen den verschiedenen Massagegriffen wird eine Ausstreichung beider Rückenseiten durchgeführt

8. Nun Klopfung (Klatschung) der gesamten Rückenmuskulatur

9. Abschluss immer durch eine ableitende Ausstreichung. 


\subsection{Statistische Verfahren}

Für die Datenanalyse wurde das Statistikprogram SPSS 16.0 verwendet. Der arithmetische Mittelwert und die Standardabweichung fungieren für die deskriptive Beschreibung der Daten und werden tabellarisch dargestellt. Die Responderrate und die Differenz der Hauptparameter jeder Gruppe wurden in Prozent oder als Mittelwert bewertet. Unterschiede in den Hauptparametern zwischen allen drei Gruppen wurden mittels Chi2-Test oder Kruskal-Wallis-Analyse (Mann-Whitney-U-Test als post-hoc Test) geprüft.

Zu Überprüfung der Parameter im Zeitverlauf innerhalb der einzelnen Gruppen fand der Friedman-Test für k verbundene Stichproben Anwendung.

Für die Auswertung der Begleitsymptome, der Schmerzintensität und der psychometrischen Daten wurden die Unterschiede mittels prozentualer Differenz der Mittelwerte im Vergleich zur Baseline dargestellt. Als signifikant wurde ein $p<0,05$ akzeptiert. 


\section{Ergebnisse}

\subsection{Allgemeine Daten bei Baseline-Erhebung}

\subsubsection{Demographische Daten}

Die Probanden unterscheiden sich weder in der Altersverteilung noch in der Geschlechtsverteilung untereinander, insgesamt wurden Frauen ungefähr acht bis neun Mal häufiger aufgenommen als Männer. Sowohl der BMI, die Häufigkeit der Migräne ohne Aura als auch die Dauer der Migräne (Migräne-Jahre) sind in den drei Gruppen vergleichbar (s. Tabelle 13). Die Häufigkeit von Spannungskopfschmerzen in der KM-Gruppe ist um 30\% geringer als in der LD-Gruppe oder in der WG. Die Häufigkeit der Migräne mit Aura ist in der WG- zweimal häufiger als in der LD-Gruppe und in der KM-Gruppe dreimal häufiger als in der LD-Gruppe, ein signifikanter Unterschied in der Verteilung besteht jedoch nicht. Die demographischen Daten aller drei Gruppen sind der Tabelle 13 zu entnehmen.

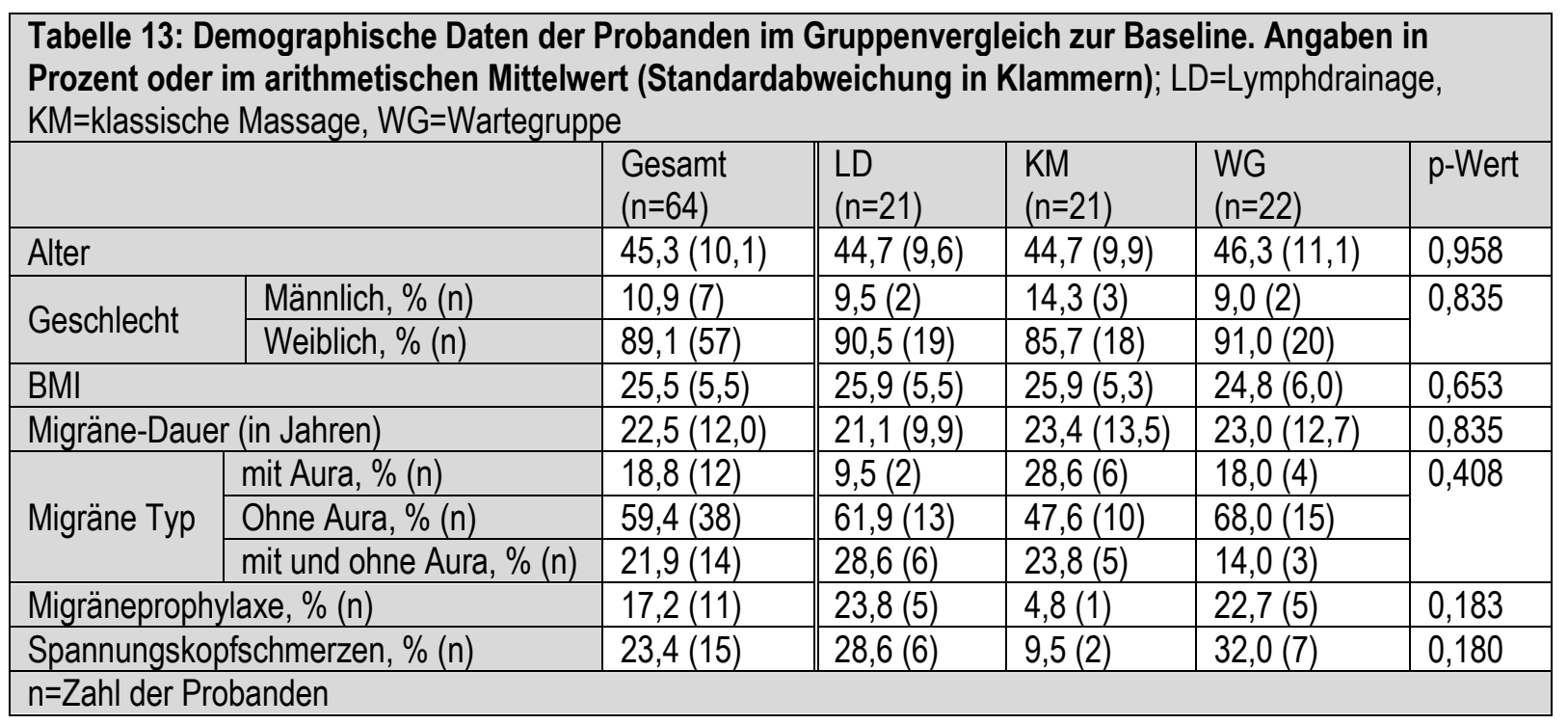

\subsubsection{Hauptparameter}

Die Hauptparameter sind (s. 3.4. Messparameter) Kopfschmerztage (KS-Tage), Migränetage, Migräneattacken und Anzahl der akut eingenommenen Medikamente (eingenommene Tabletten bei allen Migräneattacken im Monat). Alle vier Parameter waren zur Baseline in den drei Gruppen vergleichbar (s. Tabelle 14).

\begin{tabular}{|c|c|c|c|c|}
\hline & & & & \\
\hline & LD & KM & WG & $p$-Wert \\
\hline Kopfschmerztage, $n$ & $8,6(4,5)$ & $8,1(5,1)$ & $6,5(3,2)$ & 0,395 \\
\hline Migränetage, $\mathrm{n}$ & $8,0(4,4)$ & $7,6(4,5)$ & $5,9(2,6)$ & 0,354 \\
\hline Migräneattacken, $\mathrm{n}$ & $4,5(2,0)$ & $4,3(1,7)$ & $3,9(1,9)$ & 0,734 \\
\hline Anzahl der Akutmedikamente, $\mathrm{n}$ & $8,8(5,2)$ & $6,7(5,6)$ & $6,6(3,1)$ & 0,177 \\
\hline
\end{tabular}




\subsubsection{Begleitsymptome, Schmerzintensität und psychometrische Parameter}

Die Begleitsymptome einer Migräneattacke sind Übelkeit, Erbrechen, Photophobie und Phonophobie. Hier zeigt sich für die Begleitsymptome Übelkeit und Erbrechen sowie für die Schmerzintensität kein Unterschied zwischen den drei Gruppen zur Baseline während Photo- und Phonophobie signifikant unterschiedlich häufig auftreten mit einem geringeren Auftreten in der Wartegruppe (s. Tabelle 15).

\begin{tabular}{|c|c|c|c|c|}
\hline \multicolumn{5}{|c|}{$\begin{array}{l}\text { Tabelle 15: Begleitsymptome zur Baseline, angegeben als arithmetischer Mittelwert } \\
\text { (Standardabweichung in Klammern); LD=Lymphdrainage, KM=klassische Massage, } \\
\text { WG=Wartegruppe }\end{array}$} \\
\hline & LD & KM & WG & $p$-Wert \\
\hline Übelkeit & $3,1(3,6)$ & $2,8(3,0)$ & $2,5(2,0)$ & 0,938 \\
\hline Erbrechen & $0,4(1,0)$ & $0,3(0,6)$ & $0,4(0,7)$ & 0,692 \\
\hline Photophobie & $6,3(5,0)$ & $5,0(4,9)$ & $3,3(3,0)$ & 0,048 \\
\hline Phonophobie & $3,7(3,2)$ & $4,3(4,5)$ & $1,7(2,6)$ & 0,023 \\
\hline Schmerzintensität & $2,5(0,5)$ & $2,2(0,5)$ & $2,3(0,5)$ & 0,135 \\
\hline
\end{tabular}

Die psychometrischen Parameter bestehen aus akuter Beeinträchtigung durch Kopfschmerzen (emotional und körperlich), gemessen mit dem IBK (s. 3.2.4.1), Einschränkungen der Leistungsfähigkeit, gemessen mit dem Midas (3.4.2.2), depressiven Symptomen, gemessen mit der CES-D (3.4.2.3), Lebensqualität, gemessen mit dem SF-36 in acht Subskalen (s. Tabelle 16) sowie zwei Summenskalen (3.4.2.4), Schlafqualität, gemessen mit dem PSQI (3.4.2.5), und objektiver Symptomschwere, gemessen mit dem CGI (3.4.2.6). Außer der Einschränkung der Leistungsfähigkeit (Midas) in der LDGruppe $(40,5 \pm 32,3$; bei einem Ausreißer von 164) ist zur Baseline kein signifikanter Unterschied zwischen den Gruppen zu beobachten (s. Tabelle 17).

\begin{tabular}{|c|c|c|c|c|c|}
\hline \multicolumn{6}{|c|}{$\begin{array}{l}\text { Tabelle 16: Subskalenwerte des SF-36 zur Baseline, angegeben als arithmetischer Mittelwert } \\
\text { (Standardabweichung in Klammern); LD=Lymphdrainage, KM=klassische Massage, } \\
\text { WG=Wartegruppe }\end{array}$} \\
\hline & LD & KM & WG & $p$-Wert & Migräniker* \\
\hline KÖFU & $80,4(17,8)$ & $81,0(20,69$ & $81,8(16,3)$ & 0,861 & $82,9(20,3)$ \\
\hline KÖRO & $42,3(33,2)$ & $50,0(37,1)$ & $42,6(30,6)$ & 0,749 & $54,1(38,4)$ \\
\hline $\mathrm{SCHM}$ & $33,6(16,9)$ & $37,1(11,7)$ & $38,7(16,7)$ & 0,550 & $32,1(20,2)$ \\
\hline AGES & $60,5(16,5)$ & $67,0(20,2)$ & $56,6(19,7)$ & 0,123 & $57,2(19,8)$ \\
\hline VITA & $50,2(15,8)$ & $50,7(16,1)$ & $49,8(12,5)$ & 0,990 & $49,3(17,4)$ \\
\hline SOFU & $57,1(25,5)$ & $67,9(18,4)$ & $66,2(18,0)$ & 0,241 & $63,6(22,5)$ \\
\hline EMRO & $63,5(37,9)$ & $69,8(42,0)$ & $65,4(42,3)$ & 0,760 & $69,2(40,3)$ \\
\hline PSYC & $57,3(18,0)$ & $67,8(15,8)$ & $62,3(15,3)$ & 0,134 & $60,9(17,9)$ \\
\hline \multicolumn{6}{|c|}{$\begin{array}{l}\text { KÖFU: Körperliche Funktionsfähigkeit, KÖRO: Körperliche Rollenfunktion, SCHM: Körperliche } \\
\text { Schmerzen, AGES: Allgemeine Gesundheitswahrnehmung, VITA: Vitalität, SOFU: Soziale } \\
\text { Funktionsfähigkeit, EMRO: Emotionale Rollenfunktion, PSYC: Psychisches Wohlbefinden. } \\
\text { * Werte aus Bullinger und Kirchberger 1998, S. } 34\end{array}$} \\
\hline
\end{tabular}




\begin{tabular}{|c|c|c|c|c|}
\hline \multicolumn{5}{|c|}{$\begin{array}{l}\text { Tabelle 17: Psychometrische Parameter zur Baseline, angegeben als arithmetischer Mittelwert } \\
\text { (Standardabweichung in Klammern); LD=Lymphdrainage, KM=klassische Massage, WG=Wartegruppe }\end{array}$} \\
\hline & LD & KM & WG & $p$-Wert \\
\hline IBK & $64,2(12,9)$ & $58,7(19,8)$ & $54,7(16,8)$ & 0,101 \\
\hline Midas & $40,5(32,3)$ & $22,4(15,7)$ & $20,6(13,1)$ & 0,004 \\
\hline CES-D & $14,9(8,3)$ & $14,0(8,5)$ & $15,1(7,7)$ & 0,843 \\
\hline $\begin{array}{l}\text { SF-36 (körperliche } \\
\text { Summenskala) }\end{array}$ & 41,9 & 42,3 & 41,4 & 0,560 \\
\hline $\begin{array}{l}\text { SF-36 (psychische } \\
\text { Summenskala) }\end{array}$ & 42,3 & 46,7 & 44,5 & 0,467 \\
\hline PSQI gesamt & $6,7(2,9)$ & $6,3(3,2)$ & $7,3(3,3)$ & 0,777 \\
\hline CGI gesamt & $2,0(0,9)$ & $1,8(0,6)$ & $1,9(0,5)$ & 0,684 \\
\hline
\end{tabular}

\subsection{Veränderung der Parameter im Verlauf der Untersuchung in der LD-Gruppe}

\subsubsection{Veränderung der Hauptparameter}

In der LD-Gruppe fällt eine Abnahme im Behandlungsverlauf bezüglich sämtlicher Hauptparameter auf:

- Kopfschmerztage vier Wochen nach Therapie um 20\%, acht Wochen nach Therapie (definiert als sekundärer Endpunkt) um 33\% und Nachbeobachtung (definiert als primärer Endpunkt) um $36 \%$.

- $\quad$ Migränetage vier Wochen nach Therapie um 25\%, acht Wochen nach Therapie (definiert als sekundärer Endpunkt) um 33\% und Nachbeobachtung (definiert als primärer Endpunkt) um $39 \%$.

- $\quad$ Migräneattacken vier Wochen nach Therapie um 11\%, acht Wochen nach Therapie (definiert als sekundärer Endpunkt) um 33\% und Nachbeobachtung (definiert als primärer Endpunkt) um $40 \%$.

- Anzahl der akuten Medikation vier Wochen nach Therapie um 22\%, acht Wochen nach Therapie (definiert als sekundärer Endpunkt) um 39\% und Nachbeobachtung (definiert als primärer Endpunkt) um 40\% (s. Tabelle 18 und Abbildung 5).

\begin{tabular}{|c|c|c|c|c|c|}
\hline \multicolumn{6}{|c|}{$\begin{array}{l}\text { Tabelle 18: Hauptparameter im Verlauf der Untersuchung in der LD-Gruppe, angegeben als } \\
\text { arithmetischer Mittelwert (Standardabweichung in Klammern), Friedman-Test }\end{array}$} \\
\hline & Baseline & 4 Wochen & 8 Wochen & Nachbeobachtung & $p$-Wert \\
\hline Kopfschmerztage & $8,6(4,5)$ & $6,9(3,4)$ & $5,8(3,6)$ & $5,5(3,4)$ & 0,036 \\
\hline Migränetage & $8,0(4,4)$ & $6,0(2,6)$ & $5,4(3,4)$ & $4,9(3,2)$ & 0,002 \\
\hline Migräneattacken & $4,5(2,0)$ & $4,0(1,7)$ & $3,0(1,6)$ & $2,7(1,9)$ & 0,001 \\
\hline $\begin{array}{l}\text { Anzahl der } \\
\text { Akutmedikamente }\end{array}$ & $8,8(5,2)$ & $6,9(4,6)$ & $5,4(4,4)$ & $5,3(4,0)$ & 0,004 \\
\hline
\end{tabular}




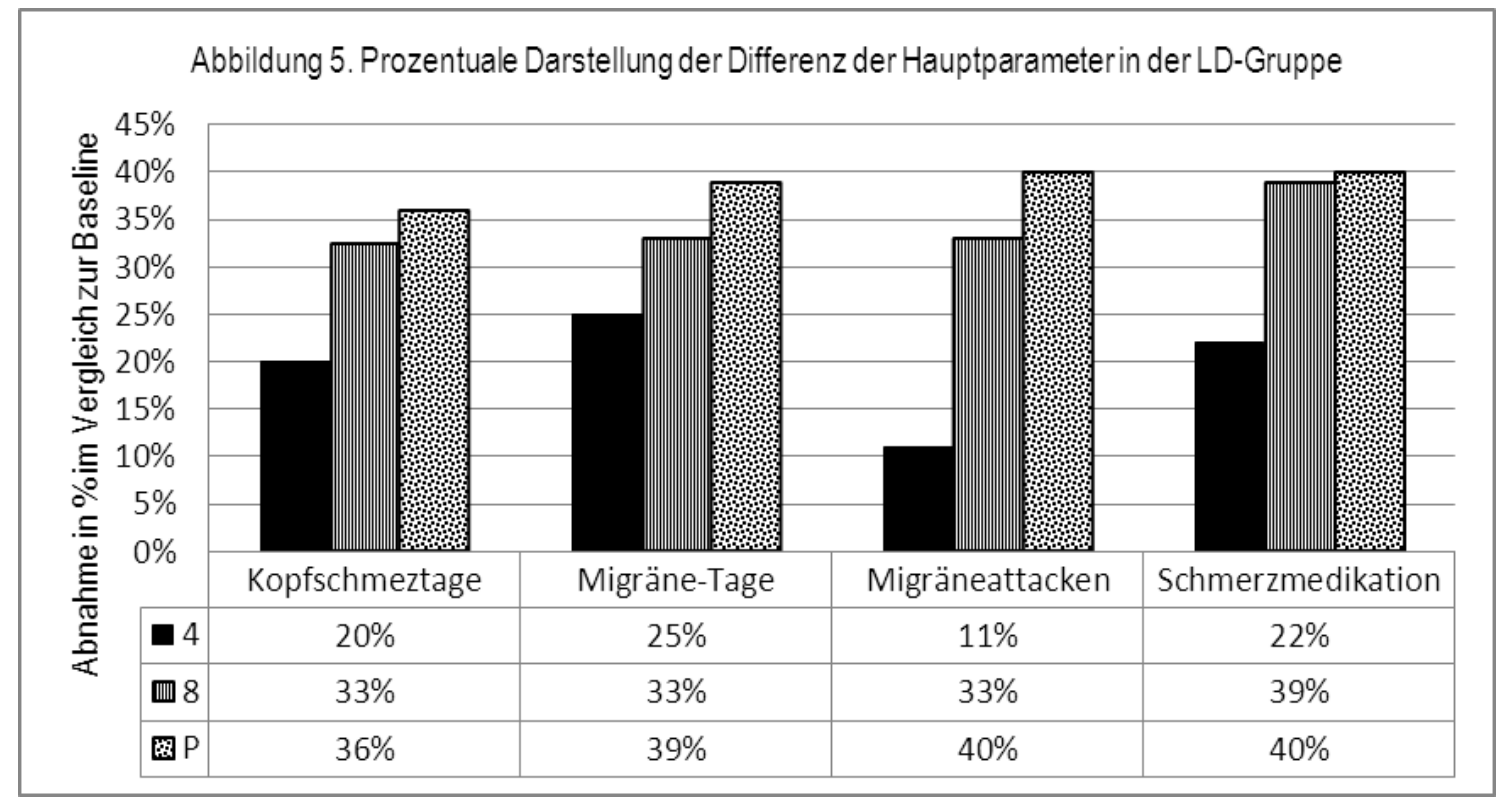

\subsubsection{Veränderung der Begleitsymptome und der Schmerzintensität}

In der LD-Gruppe fält eine Abnahme bezüglich folgender Begleitsymptome auf:

- Übelkeit vier Wochen nach Therapie um 29\%, acht Wochen nach Therapie um 32\% und Nachbeobachtung um $48 \%$.

- Erbrechen acht Wochen nach Therapie um 25\% und Nachbeobachtung um 50\%. Hier war keine Veränderung nach 4 Wochen Therapie zu beobachten

- Photophobie vier Wochen nach Therapie um 30\%, acht Wochen nach Therapie um 49\% und Nachbeobachtung um 38\%.

- Phonophobie vier Wochen nach Therapie um 11\%, acht Wochen nach Therapie um $41 \%$ und Nachbeobachtung um 38\%.

- Die Schmerzintensität vier Wochen nach Therapie um 16\%, acht Wochen nach Therapie um $28 \%$ und Nachbeobachtung um 28\% (s. Tabelle 19 und Abbildung 6).

Statistisch signifikant waren dabei die Abnahme der Photophobie und der Schmerzintensität im Verlauf.

Tabelle 19: Begleitsymptome und Schmerzintensität im Verlauf der Untersuchung in der LDGruppe, angegeben als arithmetischer Mittelwert (Standardabweichung in Klammern), FriedmanTest

\begin{tabular}{|l|l|l|l|l|l|}
\hline & Baseline & 4 Wochen & 8 Wochen & Nachbeobachtung & $p$-Wert \\
\hline Übelkeit & $3,1(3,6)$ & $2,2(2,3)$ & $2,1(2,7)$ & $1,6(2,0)$ & 0,291 \\
\hline Erbrechen & $0,4(1,0)$ & $0,4(1,0)$ & $0,3(0,7)$ & $0,2(0,5)$ & 0,812 \\
\hline Photophobie & $6,3(5,0)$ & $4,4(3,4)$ & $3,2(3,3)$ & $3,9(3,2)$ & $\mathbf{0 , 0 0 1}$ \\
\hline Phonophobie & $3,7(3,2)$ & $3,3(3,1)$ & $2,2(3,0)$ & $2,3(3,0)$ & 0,156 \\
\hline Schmerzintensität & $2,5(0,5)$ & $2,1(0,7)$ & $1,8(0,9)$ & $1,8(0,9)$ & $\mathbf{0 , 0 0 1}$ \\
\hline
\end{tabular}




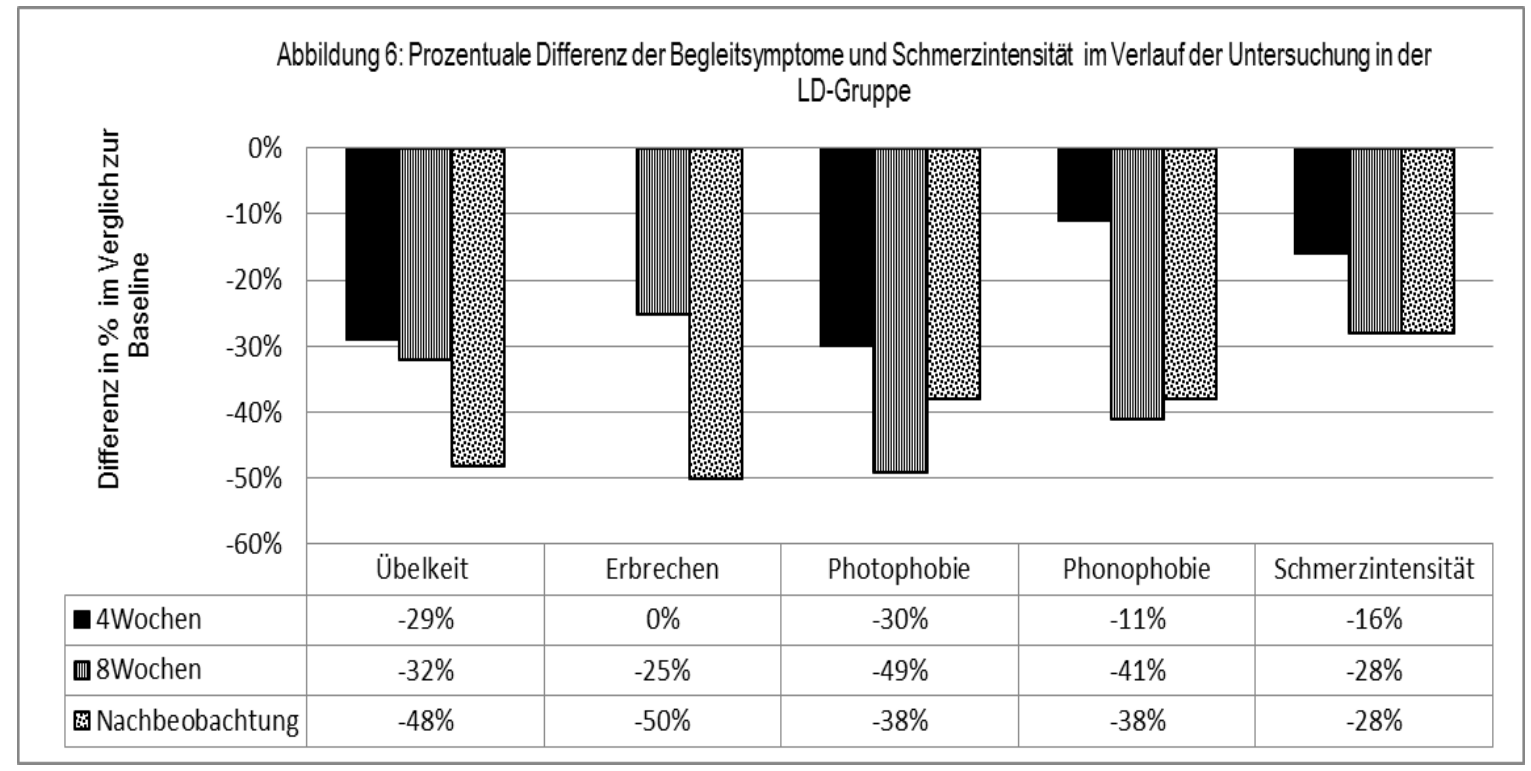

\subsubsection{Veränderung der psychometrischen Parameter}

In der LD-Gruppe fällt bezüglich der psychometrischen Parameter im Vergleich zur Baseline folgendes auf:

- eine Abnahme der Beeinträchtigung durch Kopfschmerzen gemessen durch IBK vier Wochen nach Therapie um 19\%, acht Wochen nach Therapie um 23\% und bei der Nachbeobachtung um $22 \%$.

- eine Abnahme der Einschränkungen der Leistungsfähigkeit gemessen durch Midas vier Wochen nach Therapie um 20\%, acht Wochen nach Therapie um 22\% und bei der Nachbeobachtung um $43 \%$.

- $\quad$ eine Verbesserung der Lebensqualität in beiden Summenskalen vier Wochen nach Therapie um $4 \%$ und $16 \%$, acht Wochen nach Therapie um $8 \%$ und $14 \%$, und bei der Nachbeobachtung um $5 \%$ und $16 \%$. Die Ergebnisse der Subskalen sind in Tabelle 21 dargestellt. Im Vergleich mit Baseline zeigt Abbildung 7 leichte Verbesserungen aller Subskalen 4 Wochen nach Therapie. Sie näherten sich in bestimmten Subskalen wie in Vitalität, Soziale Funktionsfähigkeit, Emotionale Rollenfunktion und Psychisches Wohlbefinden den Parametern einer Normstichprobe.

- eine Zunahme der Schlafqualität gemessen durch PSQI vier Wochen nach Therapie um 12\%, acht Wochen nach Therapie um 30\% und bei der Nachbeobachtung um 27\%.

- eine Besserung der objektiven Symptomschwere gemessen durch CGI vier Wochen nach Therapie um 30\%, acht Wochen nach Therapie um 50\% und bei der Nachbeobachtung um $45 \%$.

- $\quad$ eine Abnahme der depressiven Symptome gemessen durch CES-D vier Wochen nach Therapie um 19\%, acht Wochen nach Therapie um 36\% und bei der Nachbeobachtung um 25\% (s. Tabelle 20 und Abbildung 8).

Statistisch signifikant waren dabei die Abnahme des IBK, die Zunahme der körperlichen Summenskala des SF-36 und die Verbesserung des CGI im Verlauf. 
Tabelle 20: Psychometrische Parameter im Verlauf der Untersuchung in der LD-Gruppe, angegeben als arithmetischer Mittelwert (Standardabweichung in Klammern), Friedman-Test

\begin{tabular}{|l|l|l|l|l|l|}
\hline & Baseline & 4 Wochen & 8 Wochen & Nachbeobachtung & p-Wert \\
\hline IBK gesamt & $64,2(12,9)$ & $51,1(20,4)$ & $49,5(19,3)$ & $49,9(24,7)$ & $\mathbf{0 , 0 0 8}$ \\
\hline Midas & $40,5(32,3)$ & $32,2(21,5)$ & $33,0(33,1)$ & $22,9(16,6)$ & 0,057 \\
\hline CESD gesamt & $14,9(8,3)$ & $12,0(6,5)$ & $9,6(6,8)$ & $11,2(10,0)$ & 0,116 \\
\hline $\begin{array}{l}\text { SF-36 (körperliche } \\
\text { Summenskala) }\end{array}$ & 41,9 & 43,4 & 45,1 & 44,0 & $\mathbf{0 , 0 1 3}$ \\
\hline $\begin{array}{l}\text { SF-36 (psychische } \\
\text { Summenskala) }\end{array}$ & 42,3 & 49,1 & 48,4 & 49,2 & 0,068 \\
\hline PSQI gesamt & $6,7(2,9)$ & $5,5(3,0)$ & $4,7(2,3)$ & $4,9(2,3)$ & 0,094 \\
\hline CGI gesamt & $2,0(0,9)$ & $1,4(0,5)$ & $1,0(0,2)$ & $1,1(0,5)$ & $\mathbf{0 , 0 0 1}$ \\
\hline $\begin{array}{l}\text { IBK: Inventar zur Beeinträchtigung durch Kopfschmerzen, Midas: Migraine disability assessment, CES-D: } \\
\text { Center for Epidemiologic Studies Depression Scale, SF-36: Lebensqualitätsfragebogen, PSQI: Pittsburgh } \\
\text { Sleep Quality Index, CGI: Clinical Global Impression }\end{array}$ \\
\hline
\end{tabular}

Tabelle 21:Veränderung der Subskalen des SF-36 im Verlauf der Untersuchung in der LD-Gruppe, angegeben als arithmetischer Mittelwert (Standardabweichung in Klammern) im Vergleich mit einer Normstichprobe ("nach Bullinger und Kirchberger 1998, S. 33), Friedman-Test

\begin{tabular}{|l|l|l|l|l|l||l|}
\hline & Baseline & 4 Wochen & 8 Wochen & Nachbeobachtung & p-Wert & Normstichprobe \\
\hline KÖFU & $80,5(17,8)$ & $87,8(13,4)$ & $87,1(13,2)$ & $86,1(11,8)$ & 0,522 & $83,3(19,7)$ \\
\hline KÖRO & $42,3(33,2)$ & $63,8(36,7)$ & $72,4(28,7)$ & $68,1(35,2)$ & $\mathbf{0 , 0 2 0}$ & $77,8(33,6)$ \\
\hline SCHM & $33,6(16,9)$ & $41,2(18,8)$ & $47,2(23,2)$ & $47,8(28,0)$ & $\mathbf{0 , 0 0 5}$ & $69,8(28,0)$ \\
\hline AGES & $60,5(16,5)$ & $66,9(13,5)$ & $64,5(15,3)$ & $60,8(15,4)$ & 0,242 & $61,1(19,7)$ \\
\hline VITA & $50,2(15,8)$ & $55,0(15,3)$ & $59,7(16,7)$ & $57,5(14,5)$ & 0,193 & $58,3(17,6)$ \\
\hline SOFU & $57,1(25,5)$ & $75,0(23,3)$ & $73,7(18,6)$ & $77,1(19,8)$ & $\mathbf{0 , 0 1 9}$ & $85,3(19,9)$ \\
\hline EMRO & $63,5(37,9)$ & $85,0(22,9)$ & $82,5(30,2)$ & $85,2(26,1)$ & 0,094 & $88,1(27,6)$ \\
\hline PSYC & $57,3(18,0)$ & $69,3(13,0)$ & $68,6(14,2)$ & $68,7(18,4)$ & 0,056 & $70,2(17,4)$ \\
\hline
\end{tabular}

KÖFU: Körperliche Funktionsfähigkeit, KÖRO: Körperliche Rollenfunktion, SCHM: Körperliche Schmerzen, AGES: Allgemeine Gesundheitswahrnehmung, VITA: Vitalität, SOFU: Soziale Funktionsfähigkeit, EMRO:

Emotionale Rollenfunktion, PSYC: Psychisches Wohlbefinden. 

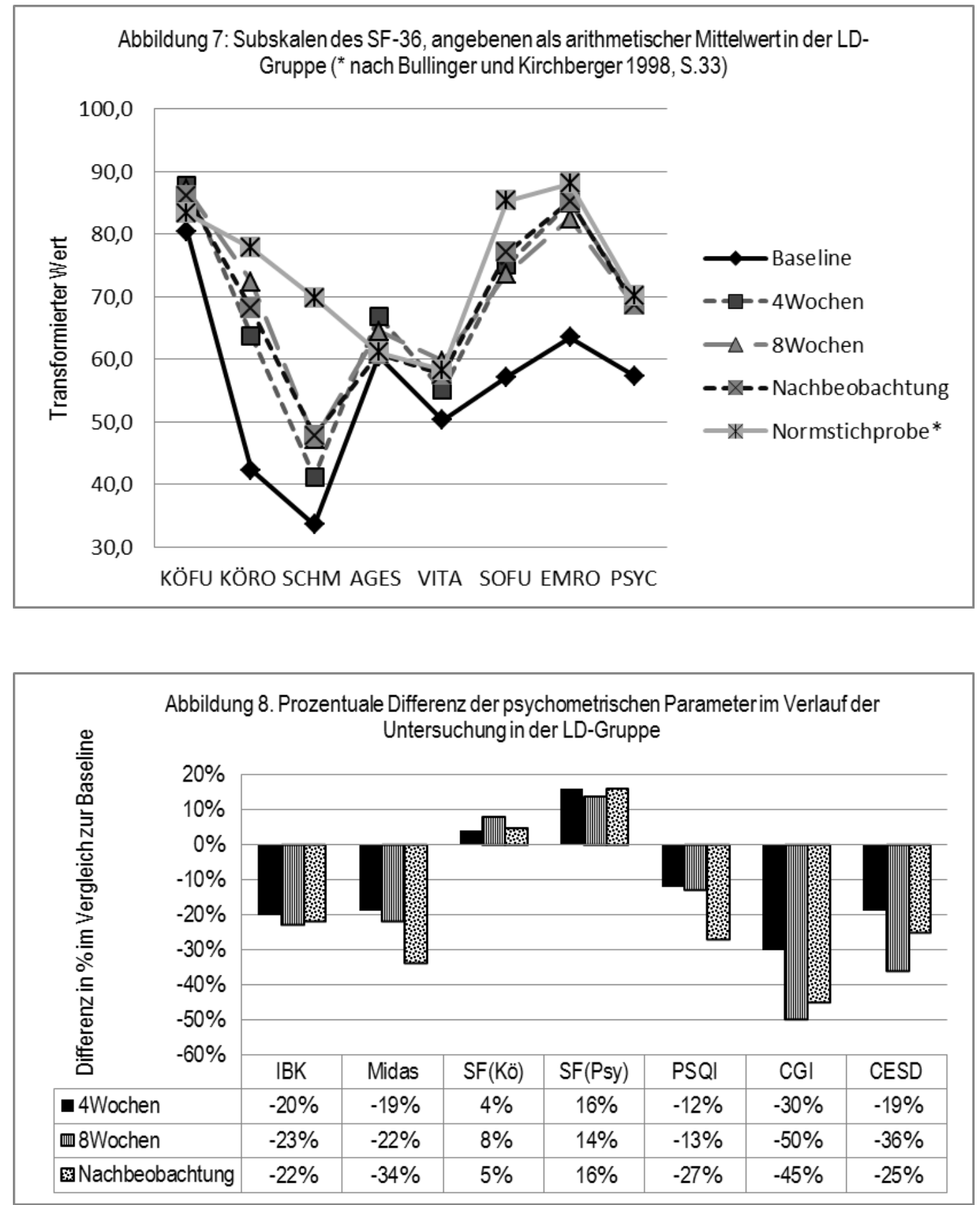

\subsection{Veränderung der Parameter im Verlauf der Untersuchung in der KM-Gruppe}

\subsubsection{Veränderung der Hauptparameter}

In der KM-Gruppe fällt eine Abnahme bezüglich folgender Hauptparameter auf:

- Kopfschmerztage vier Wochen nach Therapie um 14\%, acht Wochen nach Therapie (definiert als sekundärer Endpunkt) um 17\% und Nachbeobachtung (definiert als primärer Endpunkt) um $35 \%$. 
- $\quad$ Migränetage vier Wochen nach Therapie um 11\%, acht Wochen nach Therapie (definiert als sekundärer Endpunkt) um 18\% und Nachbeobachtung (definiert als primärer Endpunkt) um $32 \%$.

- $\quad$ Migräneattacken vier Wochen nach Therapie um 14\%, acht Wochen nach Therapie (definiert als sekundärer Endpunkt) um 26\% und Nachbeobachtung (definiert als primärer Endpunkt) um $30 \%$.

- Anzahl der akuten Medikation vier Wochen nach Therapie um 30\%, acht Wochen nach Therapie (definiert als sekundärer Endpunkt) um 33\% und Nachbeobachtung (definiert als primärer Endpunkt) um 31\%. (s. Tabelle 22 und Abbildung 9).

Statistisch signifikant waren dabei die Abnahme der Kopfschmerztage, der Migränetage und der Migräneattacken im Verlauf.

\begin{tabular}{|l|l|l|l|l|l|}
\hline \multicolumn{6}{|l|}{$\begin{array}{l}\text { Tabelle 22: Hauptparameter im Verlauf der Untersuchung in der KM-Gruppe, angegeben als } \\
\text { arithmetischer Mittelwert (Standardabweichung in Klammern), Friedman-Test }\end{array}$} \\
\hline & Baseline & 4 Wochen & 8 Wochen & Nachbeobachtung & p-Wert \\
\hline Kopfschmerztage & $8,1(5,1)$ & $7,0(4,5)$ & $6,7(5,0)$ & $5,3(5,7)$ & $\mathbf{0 , 0 1 8}$ \\
\hline Migränetage & $7,6(4,5)$ & $6,8(4,1)$ & $6,2(4,4)$ & $5,2(5,6)$ & $\mathbf{0 , 0 3 0}$ \\
\hline Migräneattacken & $4,3(1,7)$ & $3,7(1,9)$ & $3,2(1,8)$ & $3,0(2,3)$ & $\mathbf{0 , 0 0 2}$ \\
\hline $\begin{array}{l}\text { Anzahl der } \\
\text { Akutmedikamente }\end{array}$ & $6,7(5,6)$ & $4,7(3,7)$ & $4,5(3,2)$ & $4,6(4,0)$ & 0,214 \\
\hline
\end{tabular}

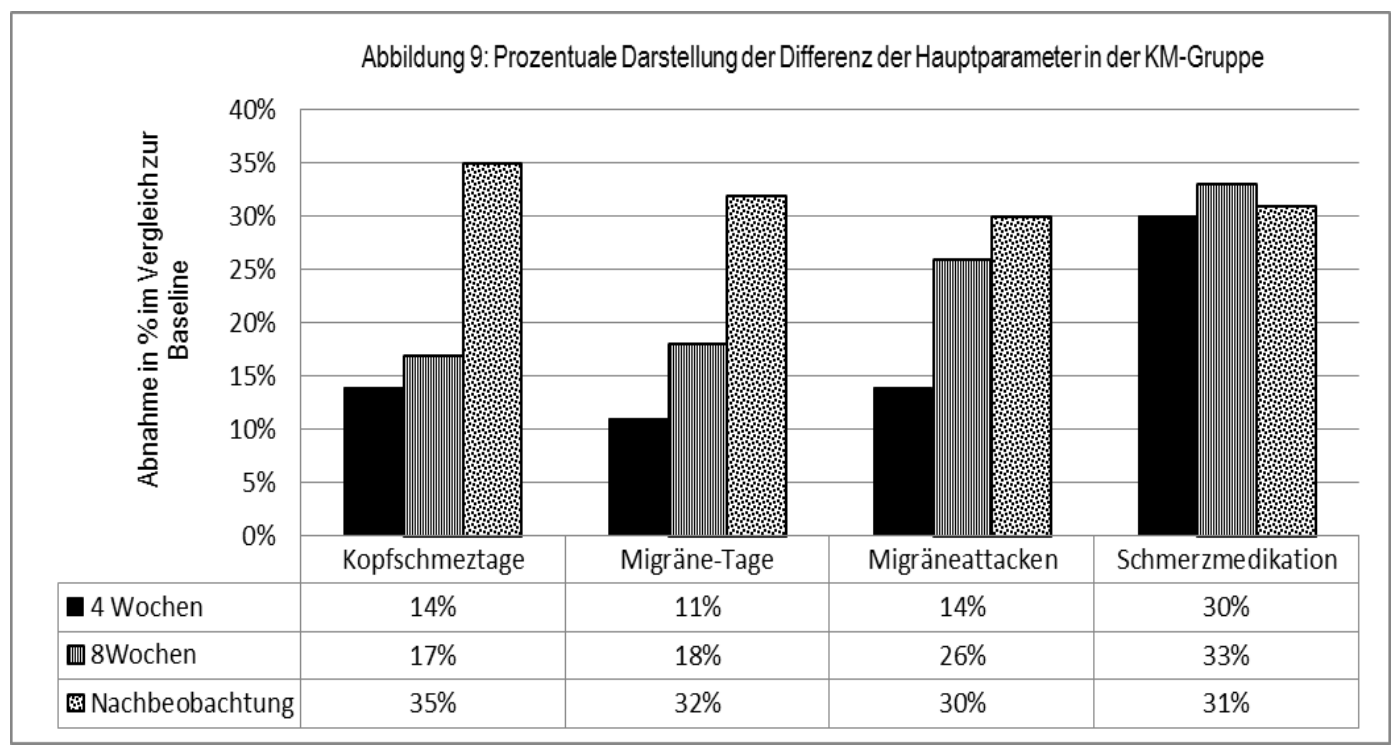

\subsubsection{Veränderung der Begleitsymptome und der Schmerzintensität}

In der KM-Gruppe fällt eine Änderung der Begleitsymptome wie folgt auf:

- Abnahme der Übelkeit vier Wochen nach Therapie um 18\%, acht Wochen nach Therapie um $39 \%$ und bei der Nachbeobachtung um 21\%.

- Zunahme des Erbrechens vier Wochen nach Therapie und bei der Nachbeobachtung jeweils um 33\%, andererseits Abnahme acht Wochen nach Therapie um 33\%. 
- $\quad$ keine Veränderung der Photophobie vier Wochen nach Therapie, Abnahme acht Wochen nach Therapie um $18 \%$ und bei der Nachbeobachtung um $24 \%$.

- Abnahme der Phonophobie vier Wochen nach Therapie um 14\%, acht Wochen nach Therapie um $21 \%$ und bei der Nachbeobachtung um 35\%.

- Abnahme der Schmerzintensität vier Wochen nach Therapie um 5\%, acht Wochen nach Therapie um 14\% und bei der Nachbeobachtung um 18\% (s. Tabelle 23 und Abbildung 10).

Statistisch signifikant war dabei die Abnahme der Photophobie im Verlauf.

\begin{tabular}{|c|c|c|c|c|c|}
\hline & Baseline & 4 Wochen & 8 Wochen & Nachbeobachtung & $p$-Wert \\
\hline Übelkeit & $2,8(3,0)$ & $2,3(3,2)$ & $1,7(2,4)$ & $2,2(3,7)$ & 0,087 \\
\hline Erbrechen & $0,3(0,6)$ & $0,4(1,4)$ & $0,2(0,7)$ & $0,4(1,2)$ & 0,552 \\
\hline Photophobie & $5,0(4,9)$ & $5,0(4,4)$ & $4,1(4,7)$ & $3,8(5,5)$ & 0,045 \\
\hline Phonophobie & $4,3(4,5)$ & $3,7(4,2)$ & $3,4(3,3)$ & $2,8(3,6)$ & 0,215 \\
\hline Schmerzintensität & $\frac{7,0(1,0)}{2,2(0,5)}$ & $2,1(1,0)$ & $1,9(0,6)$ & $1,8(0,9)$ & 0,178 \\
\hline
\end{tabular}

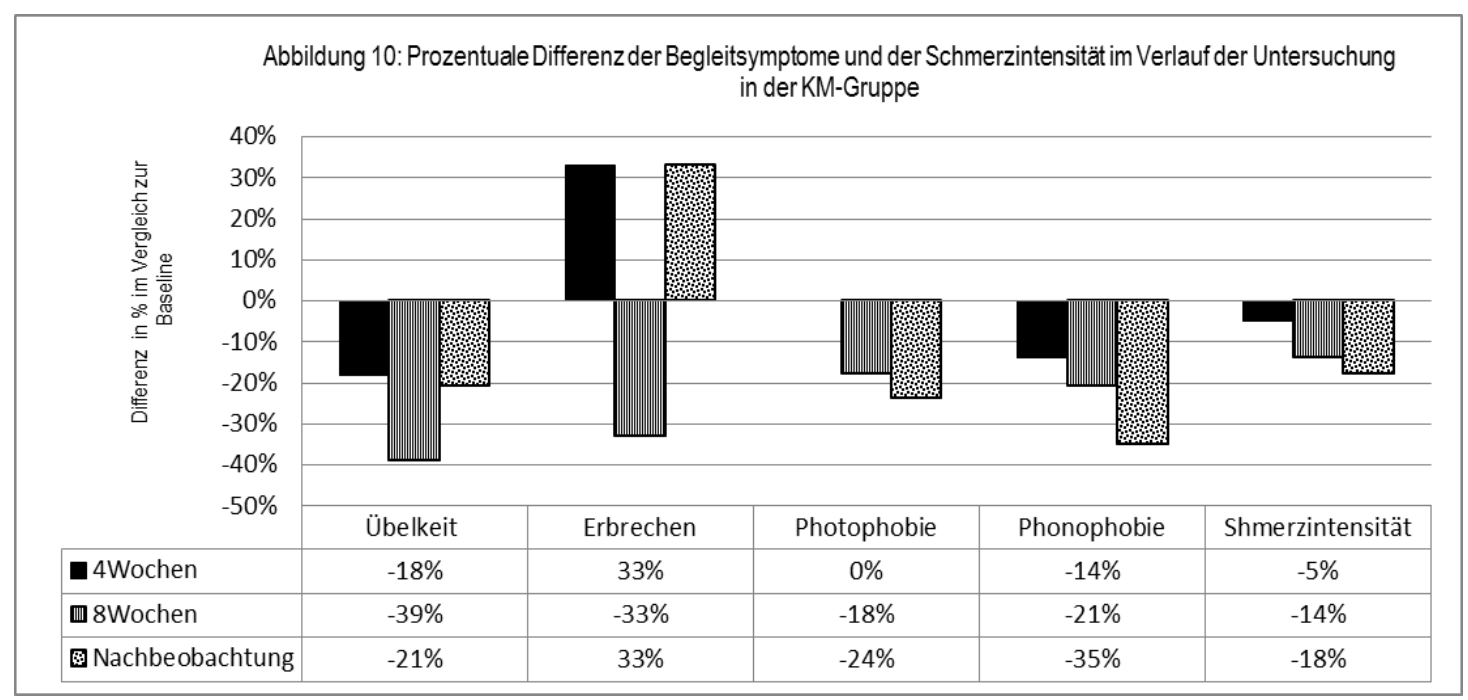

\subsubsection{Veränderung der psychometrischen Parameter}

Bezüglich der psychometrischen Parameter im Vergleich zur Baseline ist in der KM-Gruppe außer dem Midas eine signifikante Verbesserung im IBK, im CES-D, in den SF-36 Summenskalen sowie im PSQI und im CGI zu verzeichnen (s. Tabelle 24 und Abbildung 11). Abbildung 12 zeigt die Veränderungen in der Subskala des SF-36 in Vergleich mit Baseline und Normstrichprobe. Hier werden signifikante Verbesserungen in den Subskalen körperliche Funktionsfähigkeit, körperliche Rollenfunktion, Vitalität und soziale Funktionsfähigkeit im Verlauf beobachtet (s. Tabelle 25). 


\begin{tabular}{|l|l|l|l|l|l|}
\hline \multicolumn{6}{|l|}{$\begin{array}{l}\text { Tabelle 24: Psychometrische Parameter im Verlauf der Untersuchung in der KM-Gruppe, } \\
\text { angegeben als arithmetischer Mittelwert (Standardabweichung in Klammern), Friedman-Test }\end{array}$} \\
\hline & Baseline & 4 Wochen & 8 Wochen & Nachbeobachtung & p-Wert \\
\hline IBK gesamt & $58,7(19,8)$ & $54,5(22,7)$ & $49,5(20,8)$ & $48,2(22,6)$ & $\mathbf{0 , 0 0 1}$ \\
\hline Midas & $22,4(15,7)$ & $22,1(21,9)$ & $23,6(22,1)$ & $21,5(27,0)$ & 0,064 \\
\hline CESD gesamt & $14(8,4)$ & $12,8(7,3)$ & $12(7,8)$ & $12,1(9,0)$ & $\mathbf{0 , 0 3 1}$ \\
\hline $\begin{array}{l}\text { SF-36 (körperliche } \\
\text { Summenskala) }\end{array}$ & 42,3 & 41,0 & 43,6 & 45,4 & $\mathbf{0 , 0 0 4}$ \\
\hline $\begin{array}{l}\text { SF-36 (psychische } \\
\text { Summenskala) }\end{array}$ & 46,7 & 50,7 & 50,0 & 48,9 & $\mathbf{0 , 0 0 1}$ \\
\hline PSQI gesamt & $6,3(3,2)$ & $5,9(2,5)$ & $4,7(2,2)$ & $5,6(3,0)$ & $\mathbf{0 , 0 4 7}$ \\
\hline CGI gesamt & $1,8(0,6)$ & $1,7(0,7)$ & $1,2(0,4)$ & $1,1(0,3)$ & $\mathbf{0 , 0 0 1}$ \\
\hline $\begin{array}{l}\text { IBK: Inventar zur Beeinträchtigung durch Kopfschmerzen, Midas: Migraine disability assessment, CES- } \\
\text { D: Center for Epidemiologic Studies Depression Scale, SF-36: Lebensqualitätsfragebogen, PSQI: } \\
\text { Pittsburgh Sleep Quality Index, CGI: Clinical Global Impression. }\end{array}$ \\
\hline
\end{tabular}

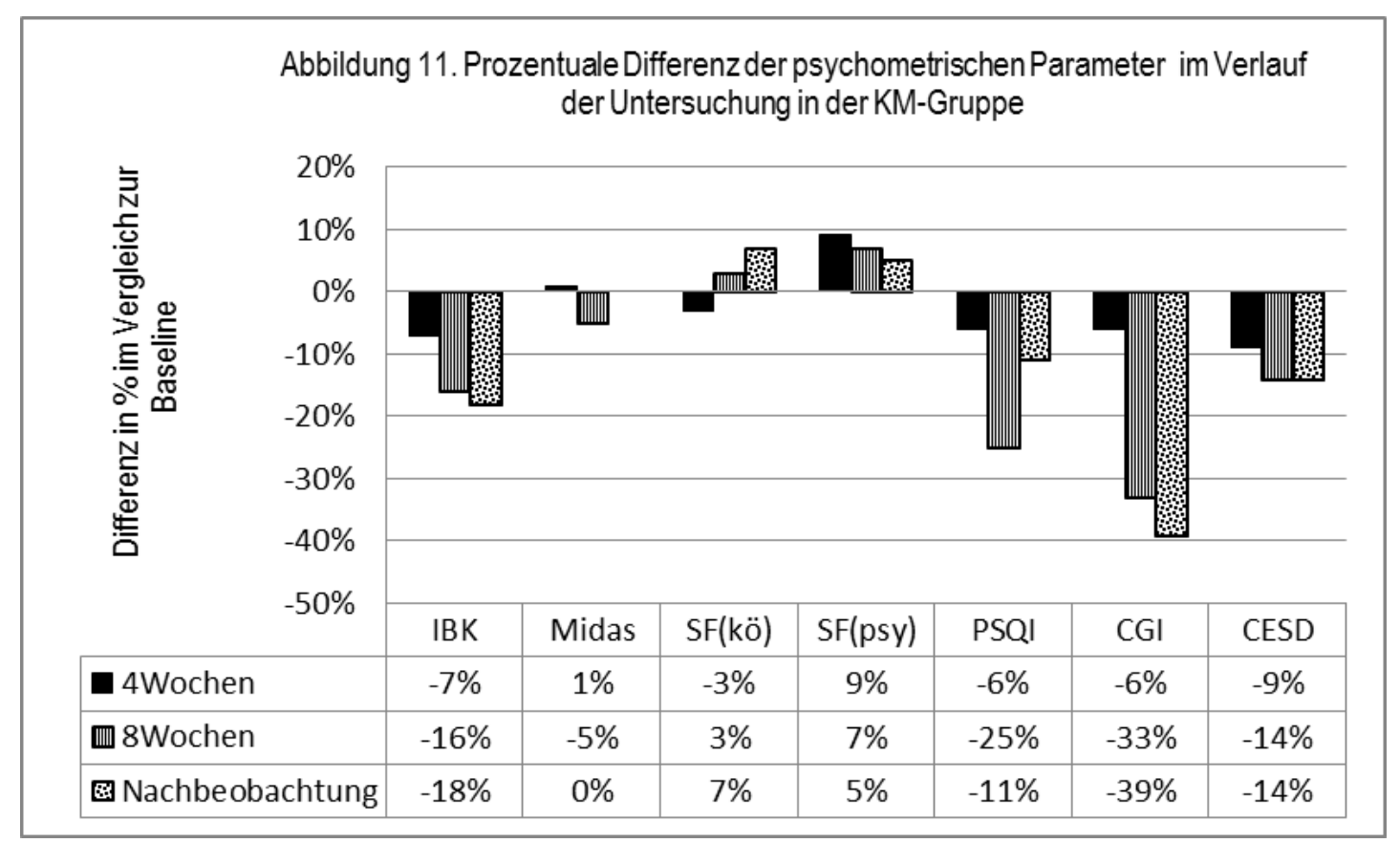




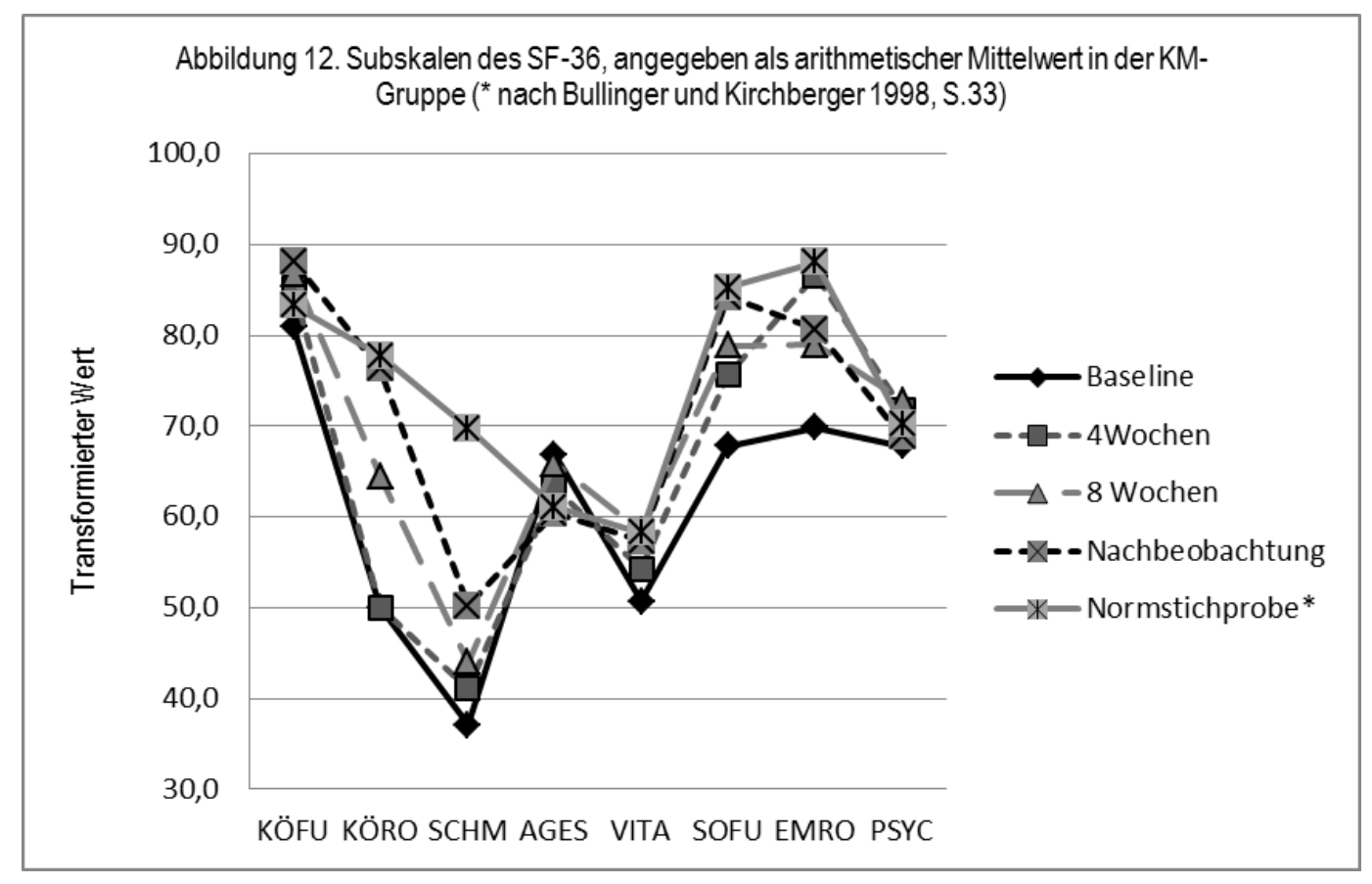

\begin{tabular}{|c|c|c|c|c|c|c|}
\hline & & & & & & \\
\hline KÖFU & $81 \cap(206)$ & $852 / 1421$ & $860 / 146)$ & $80 ?$ & 0021 & \\
\hline & & & & & $0,0<7$ & $0, v(10, r)$ \\
\hline KORO & $50,0(37,1)$ & $50,0(42,1)$ & $64,5(36,6)$ & $76,3(36,8)$ & 0,011 & $77,8(33,6)$ \\
\hline $\mathrm{SCHM}$ & $37,1(11,7)$ & $41,1(9,4)$ & $44,0(15,8)$ & $50,2(26,1)$ & 0,051 & $69,8(28,0)$ \\
\hline AGES & $66,9(20,2)$ & $63,3(20,2)$ & $65,8(16,2)$ & $60,4(20,1)$ & 0,285 & $61,1(19,7)$ \\
\hline VITA & $50,7(16,1)$ & $54,3(16,8)$ & $58,4(15,1)$ & $57,4(19,7)$ & 0,027 & $58,3(17,6)$ \\
\hline SOFU & $67,9(18,4)$ & $75,6(19,2)$ & $78,9(15,6)$ & $84,2(19,0)$ & 0,005 & $85,3(19,9)$ \\
\hline EMRO & $69,8(42,0)$ & $86,7(27,4)$ & $79,0(35,5)$ & $80,7(32.0)$ & 0,623 & $88,1(27,6)$ \\
\hline PSYC & $67,8(15,8)$ & $71,8(15,0)$ & $72,8(18,4)$ & $68,8(20,3)$ & 0,288 & $70,2(17,4)$ \\
\hline \multicolumn{7}{|c|}{$\begin{array}{l}\text { KÖFU: Körperliche Funktionsfähigkeit, KÖRO: Körperliche Rollenfunktion, SCHM: Körperliche Schmerzen, } \\
\text { AGES: Allgemeine Gesundheitswahrnehmung, VITA: Vitalität, SOFU: Soziale Funktionsfähigkeit, EMRO: } \\
\text { Emotionale Rollenfunktion, PSYC: Psychisches Wohlbefinden. }\end{array}$} \\
\hline
\end{tabular}

\subsection{Veränderung der Parameter im Verlauf der Untersuchung in der Wartegruppe (WG)}

\subsubsection{Veränderung der Hauptparameter}

In der WG-Gruppe fallen keine signifikanten Veränderungen der Hauptparameter im Vergleich zur Baseline auf. Obwohl es zu einer relativen Abnahme der Kopfschmerz- und Migränetage sowie Migräneattacken um ca. 10\% acht Wochen nach Therapie (T3) kommt, ist eine Zunahme der akuten Medikation in allen Phasen um 9\% zu beobachten (s. Tabelle 26 und Abbildung 13). Diese Unterschiede sind jedoch nicht signifikant. 


\begin{tabular}{|l|l|l|l|l|l|}
\hline $\begin{array}{l}\text { Tabelle 26: Hauptparameter im Verlauf der Untersuchung in der WG, angegeben als } \\
\text { arithmetischer Mittelwert (Standardabweichung in Klammern), Friedman-Test }\end{array}$ \\
\hline & Baseline & 4 Wochen & 8 Wochen & Nachbeobachtung & p-Wert \\
\hline Kopfschmerztage & $6,5(3,2)$ & $6,5(3,0)$ & $5,7(2,7)$ & $6,1(2,7)$ & 0,581 \\
\hline Migränetage & $5,9(2,6)$ & $6,2(2,7)$ & $5,3(2,1)$ & $5,7(2,4)$ & 0,353 \\
\hline Migräneattacken & $3,9(1,4)$ & $4,0(1,6)$ & $3,6(1,5)$ & $3,5(1,2)$ & 0,251 \\
\hline $\begin{array}{l}\text { Anzahl der } \\
\text { Akutmedikamente }\end{array}$ & $6,6(3,1)$ & $7,2(5,3)$ & $7,1(4,2)$ & $7,2(4,6)$ & 0,740 \\
\hline
\end{tabular}

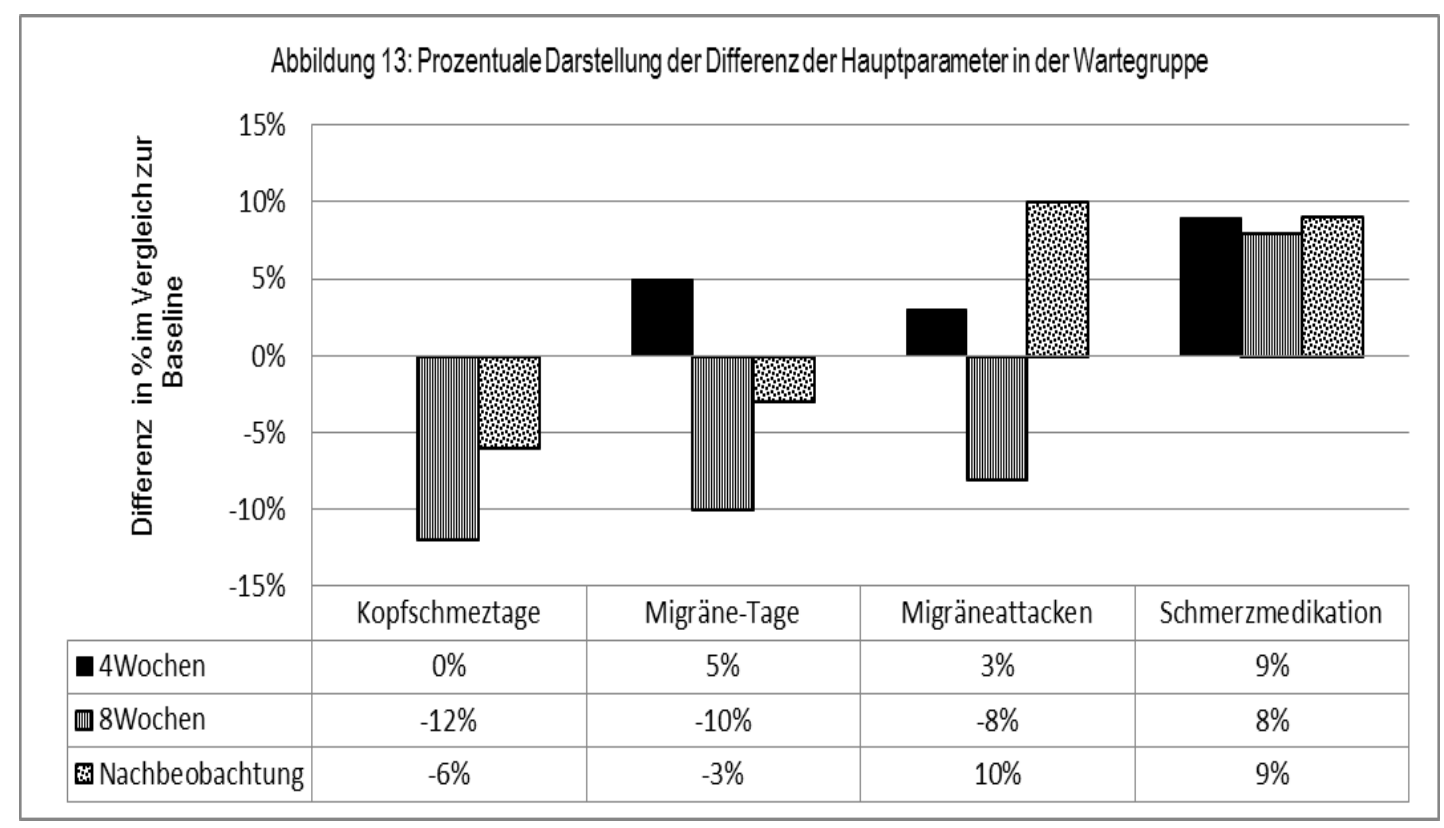

\subsubsection{Veränderung der Begleitsymptome und der Schmerzintensität}

Bezüglich der Begleitsymptome fallen in der WG folgende Änderungen auf:

- Zunahme der Übelkeit vier Wochen nach Therapie um $8 \%$ und bei der Nachbeobachtung um 4\%, Abnahme acht Wochen nach Therapie um 8\%.

- Abnahme des Erbrechens vier Wochen nach Therapie um 75\%, Zunahme um jeweils 25\% nach acht Wochen und bei der Nachbeobachtung.

- $\quad$ keine Veränderung der Photophobie vier Wochen nach Therapie, Abnahme acht Wochen nach Therapie um $21 \%$ und bei der Nachbeobachtung um 30\%.

- keine Veränderung der Phonophobie acht Wochen nach Therapie, Zunahme vier Wochen nach Therapie und bei der Nachbeobachtung um jeweils $25 \%$.

- Keine Veränderung in der Schmerzintensität im Verlauf der gesamten Zeit der Untersuchung (s. Tabelle 27 und Abbildung 14).

Im Verlauf zeigen sich bei allen diesen Parametern keine signifikanten Unterschiede. 


\begin{tabular}{|l|l|l|l|l|l|}
\hline \multicolumn{6}{|l|}{$\begin{array}{l}\text { Tabelle 27: Begleitsymptome und Schmerzintensität im Verlauf der Untersuchung in der WG, } \\
\text { angegeben als arithmetischer Mittelwert (Standardabweichung in Klammern), Friedman-Test }\end{array}$} \\
\hline & Baseline & 4 Wochen & 8 Wochen & Nachbeobachtung & p-Wert \\
\hline Übelkeit & $2,5(2,0)$ & $2,7(3,1)$ & $2,3(2,1)$ & $2,6(2,9)$ & 0,764 \\
\hline Erbrechen & $0,4(0,7)$ & $0,1(0,3)$ & $0,5(1,1)$ & $0,3(0,6)$ & 0,271 \\
\hline Photophobie & $3,3(3,0)$ & $3,3(3,1)$ & $2,6(3,0)$ & $3,2(3,3)$ & 0,997 \\
\hline Phonophobie & $1,7(2,6)$ & $2,3(3,2)$ & $1,7(3,0)$ & $2,3(3,2)$ & 0,396 \\
\hline Schmerzintensität & $2,3(0,5)$ & $2,4(1,0)$ & $2,4(0,7)$ & $2,3(0,6)$ & 0,787 \\
\hline
\end{tabular}

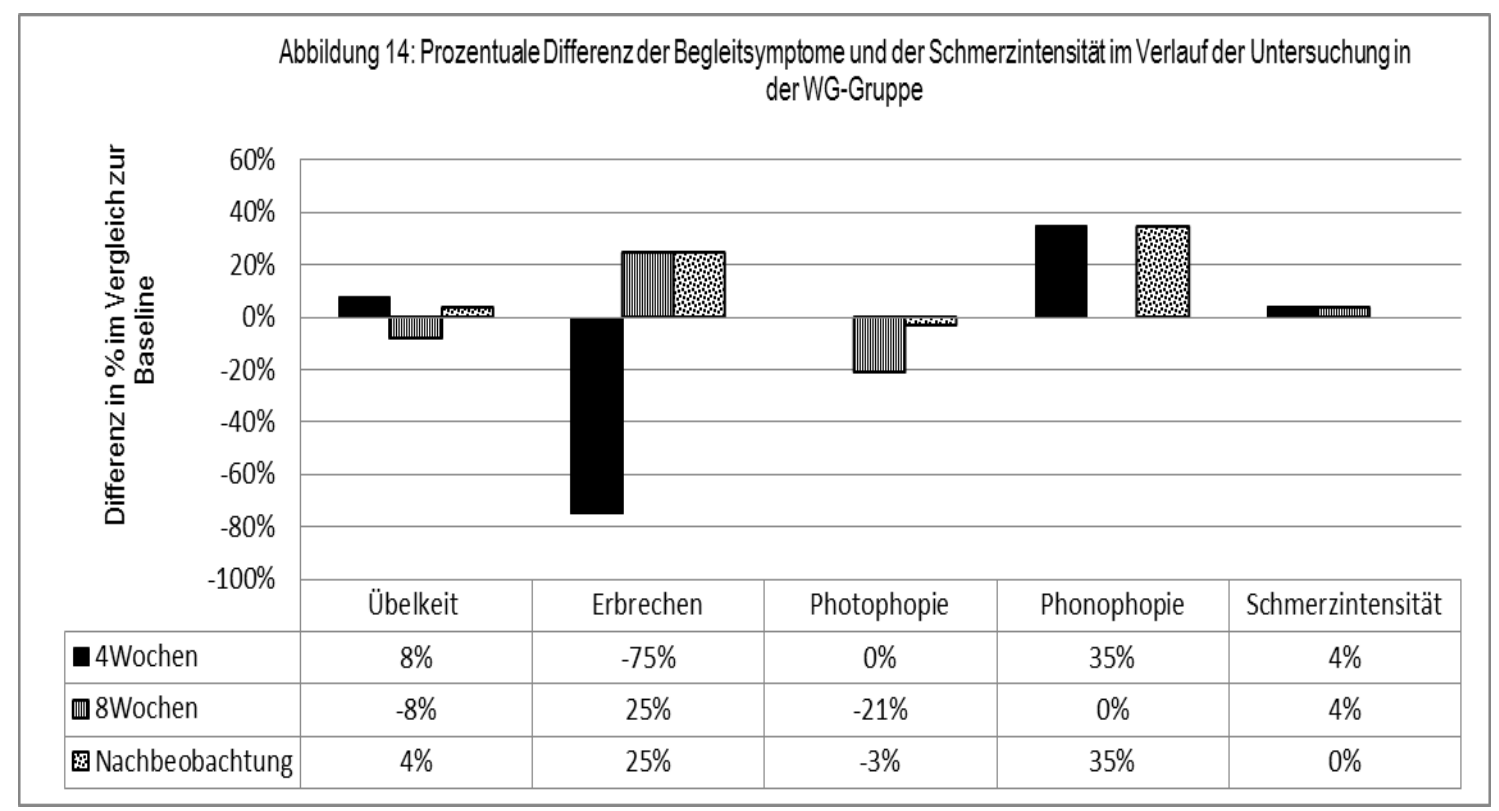

\subsubsection{Veränderung der psychometrischen Parameter}

Die psychometrischen Parameter in der WG-Gruppe zeigen keine wesentliche Veränderung außer einer Zunahme der depressiven Symptome um 11\% bzw. 14\% nach 8 Wochen bzw. bei der Nachbeobachtung und einer Abnahme der Beeinträchtigung durch Kopfschmerzen um 17\% bzw. 23\% nach 8 Wochen bzw. bei der Nachbeobachtung (s. Tabelle 28 und 29, Abbildungen 15 und 16).

Im Verlauf kommt es zu einer signifikanten Abnahme des IBK und einer signifikanten Zunahme der körperlichen Summenskala des SF-36 sowie in den Subskalen körperliche Rollenfunktion und körperliche Schmerzen. 


\begin{tabular}{|l|l|l|l|l|l|}
\hline \multicolumn{6}{|l|}{$\begin{array}{l}\text { Tabelle 28: Psychometrische Parameter im Verlauf der Untersuchung in der WG-Gruppe, } \\
\text { angegeben als arithmetischer Mittelwert (Standardabweichung in Klammern), Friedman-Test }\end{array}$} \\
\hline & Baseline & 4 Wochen & 8 Wochen & Nachbeobachtung & p-Wert \\
\hline IBK gesamt & $54,7(16,8)$ & $51,4(16,6)$ & $45,3(13,7)$ & $42,0(20,4)$ & $\mathbf{0 , 0 0 1}$ \\
\hline Midas & $20,6(13,1)$ & $25,5(14,2)$ & $22,3(16,4)$ & $19,2(16,1)$ & 0,191 \\
\hline CESD gesamt & $15,1(7,7)$ & $14,4(8,3)$ & $15,8(8,1)$ & $16,7(9,8)$ & 0,964 \\
\hline $\begin{array}{l}\text { SF-36 (körperliche } \\
\text { Summenskala) }\end{array}$ & 41,4 & 39,7 & 44,1 & 43,6 & $\mathbf{0 , 0 1 2}$ \\
\hline $\begin{array}{l}\text { SF-36 (psychische } \\
\text { Summenskala) }\end{array}$ & 44,5 & 45,8 & 42,1 & 45,0 & 0,445 \\
\hline PSQI gesamt & $7,3(3,3)$ & $7,2(3,1)$ & $6,5(3,0)$ & $6,3(3,6)$ & 0,905 \\
\hline CGI gesamt & $1,9(0,5)$ & $1,8(0,6)$ & $1,8(0,4)$ & $1,8(0,5)$ & 0,773 \\
\hline $\begin{array}{l}\text { IBK: Inventar zur Beeinträchtigung durch Kopfschmerzen, Midas: Migraine disability assessment, CES- } \\
\text { D: Center for Epidemiologic Studies Depression Scale, SF-36: Lebensqualitätsfragebogen, PSQI: } \\
\text { Pittsburgh Sleep Quality Index, CGI: Clinical Global Impression. }\end{array}$ \\
\hline
\end{tabular}

\begin{tabular}{|c|c|c|c|c|c|c|}
\hline & Baseline & 4 Wochen & 8 Wochen & Nachbeobachtung & $p$-Wert & Normstichprobe* \\
\hline KÖFU & $81,8(16,3)$ & $81,1(17,6)$ & $87,1(12,7)$ & $87,4(10,8)$ & 0,134 & $83,3(19,7)$ \\
\hline KÖRO & $42,6(30,6)$ & $35,2(28,0)$ & $57,3(27,5)$ & $58,2(29,4)$ & 0,050 & $77,8(33,6)$ \\
\hline $\mathrm{SCHM}$ & $38,7(16,7)$ & $36,5(11,1)$ & $40,3(10,7)$ & $44,0(12,2)$ & 0,039 & $69,8(28,0)$ \\
\hline AGES & $56,6(19,7)$ & $55,6(21,1)$ & $60,1(14,2)$ & $57,5(15,3)$ & 0,666 & $61,1(19,7)$ \\
\hline VITA & $49,8(12,5)$ & $50,6(13,3)$ & $47,0(14,8)$ & $52,1(12,5)$ & 0,308 & $58,3(17,6)$ \\
\hline SOFU & $66,2(18,0)$ & $61,9(23,7)$ & $66,1(16,5)$ & $71,4(16,5)$ & 0,416 & $85,3(19,9)$ \\
\hline EMRO & $65,4(42,3)$ & $74,2(31,6)$ & $58,3(40,0)$ & $66,7(39,3)$ & 0,417 & $88,1(27,6)$ \\
\hline PSYC & $62,3(15,3)$ & $62,6(17,6)$ & $62,3(16,0)$ & $65,2(18,4)$ & 0,772 & $70,2(17,4)$ \\
\hline \multicolumn{7}{|c|}{$\begin{array}{l}\text { KÖFU: Körperliche Funktionsfähigkeit, KÖRO: Körperliche Rollenfunktion, SCHM: Körperliche } \\
\text { Schmerzen, AGES: Allgemeine Gesundheitswahrnehmung, VITA: Vitalität, SOFU: Soziale } \\
\text { Funktionsfähigkeit, EMRO: Emotionale Rollenfunktion, PSYC: Psychisches Wohlbefinden. }\end{array}$} \\
\hline
\end{tabular}



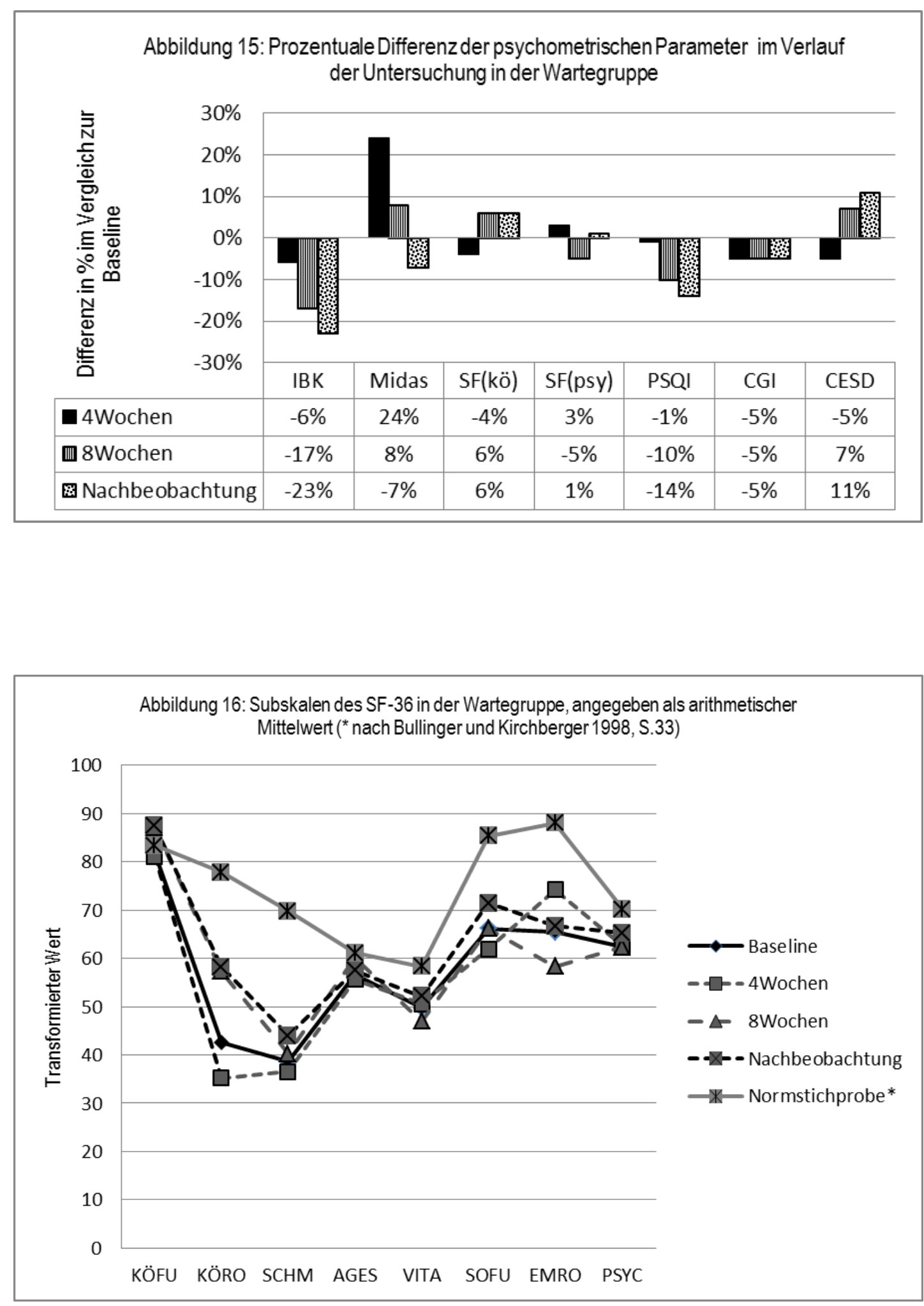


\subsection{Responderrate und Hauptparameter aller Gruppen in Vergleich}

\subsubsection{Zwischen Baseline und Nachbeobachtung (Primäre Endpunkte)}

Wie in 3.4. (Messparameter) angegeben, werden hier die Responderraten (mind. 50\%ige Reduktion der Migräneattacken und Migränetage) und die Differenz der Migräneattacken (MA), Migränetage (MT), Kopfschmerztage sowie die Differenz in der Einnahme von akuten Schmerzmedikamenten (SM) zwischen den beiden Beobachungsphasen (Baseline "T1" und Nachbeobachtung "T4=P") aller drei Gruppen als primäre Endpunkte verglichen.

Der Verlauf der Kopfschmerztage ist in Tabelle 30 und Abbildung 17 dargestellt, der Verlauf der Migränetage in Tabelle 31 sowie Abbildungen 18 und 19, der Verlauf der Migräneattacken in Tabelle 32 sowie Abbildungen 20 und 21, der Verlauf der eingenommenen Akutmedikation in Tabelle 33 sowie Abbildung 22.

Tabelle 30: Kopfschmerztage aller Gruppen zu allen Zeitpunkten, angegeben als arithmetischer Mittelwert (Standardabweichung in Klammern)

\begin{tabular}{|l|l|l|l|}
\hline & Lymphdrainage & Klassische Massage & Wartegruppe \\
\hline Baseline & $8,6(4,5)$ & $8,1(5,1)$ & $6,5(3,2)$ \\
\hline 4 Wochen & $6,9(3,4)$ & $7,0(4,5)$ & $6,5(3,0)$ \\
\hline 8 Wochen & $5,8(3,6)$ & $6,7(5,0)$ & $5,7(2,7)$ \\
\hline Nachbeobachtung & $5,5(3,4)$ & $5,3(5,7)$ & $6,1(2,7)$ \\
\hline
\end{tabular}

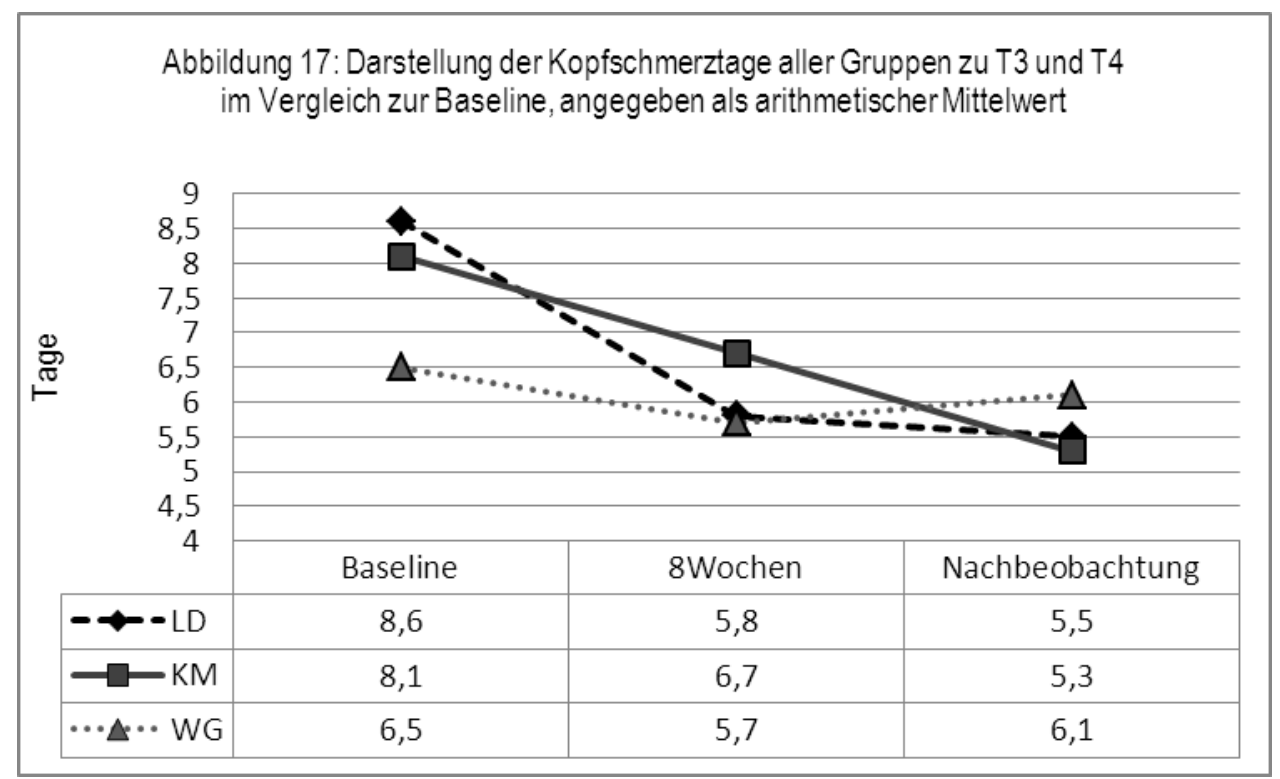




\begin{tabular}{|c|c|c|c|}
\hline & Lymphdrainage & Klassische Massage & Wartegruppe \\
\hline Baseline & $8,0(4,4)$ & $7,6(4,5)$ & $5,9(2,6)$ \\
\hline 4 Wochen & $6,0(2,6)$ & $6,8(4,1)$ & $6,2(2,7)$ \\
\hline 8 Wochen & $5,4(3,4)$ & $6,2(4,4)$ & $5,3(2,1)$ \\
\hline Nachbeobachtung & $5,5(3,4)$ & $5,3(5,7)$ & $6,1(2,7)$ \\
\hline
\end{tabular}
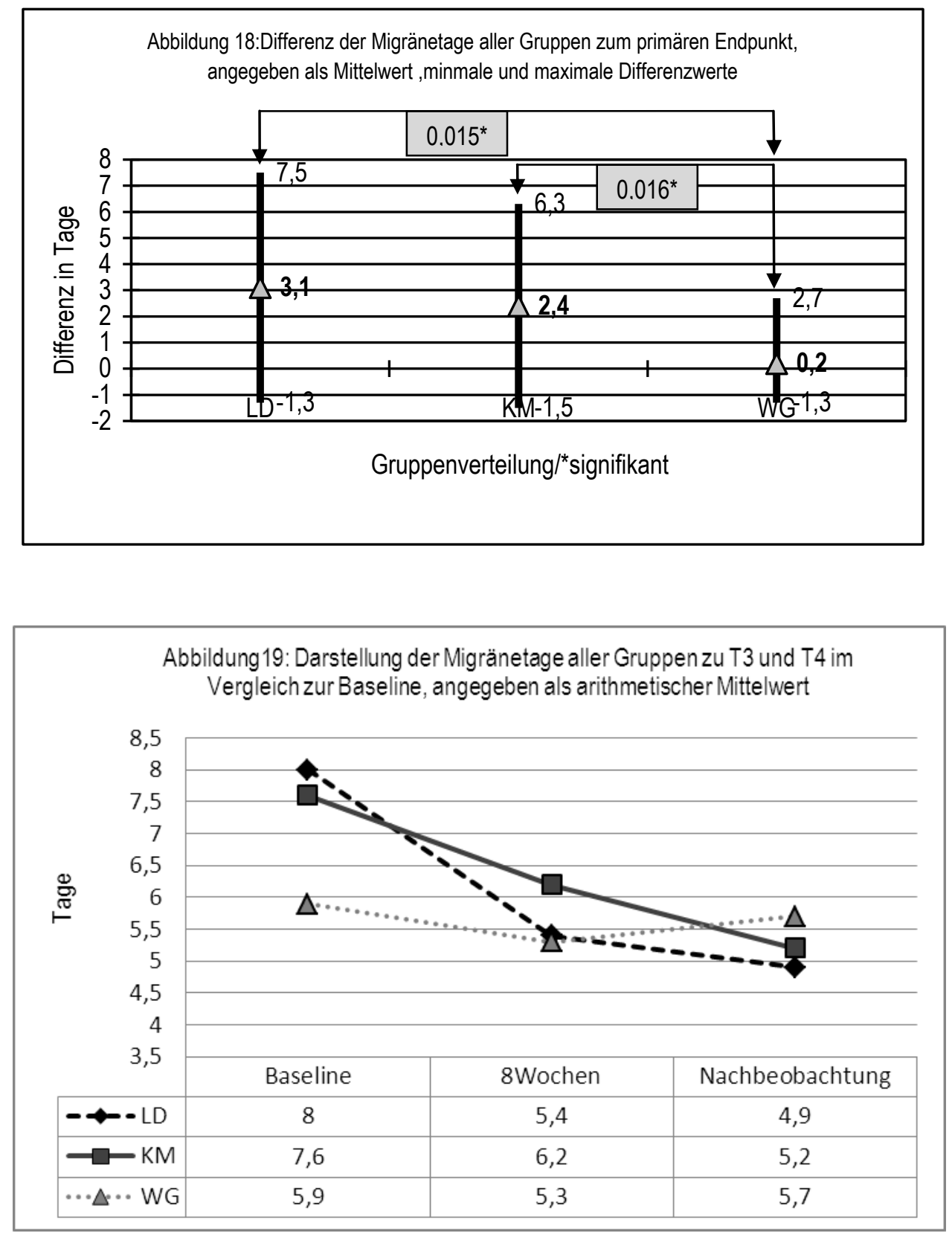


\begin{tabular}{|l|l|l|l|}
\hline \multicolumn{4}{|l|}{$\begin{array}{l}\text { Tabelle 32: Migräneattacken aller Gruppen zu allen Zeitpunkten, angegeben als } \\
\text { arithmetischer Mittelwert (Standardabweichung in Klammern) }\end{array}$} \\
\hline & Lymphdrainage & Klassische Massage & Wartegruppe \\
\hline Baseline & $4,5(2,0)$ & $4,3(1,7)$ & $3,9(1,4)$ \\
\hline 4 Wochen & $4,0(1,7)$ & $3,7(1,9)$ & $4,0(1,6)$ \\
\hline 8 Wochen & $3,0(1,6)$ & $3,2(1,8)$ & $3,6(1,5)$ \\
\hline Nachbeobachtung & $2,7(1,9)$ & $3,0(2,3)$ & $3,5(1,2)$ \\
\hline
\end{tabular}
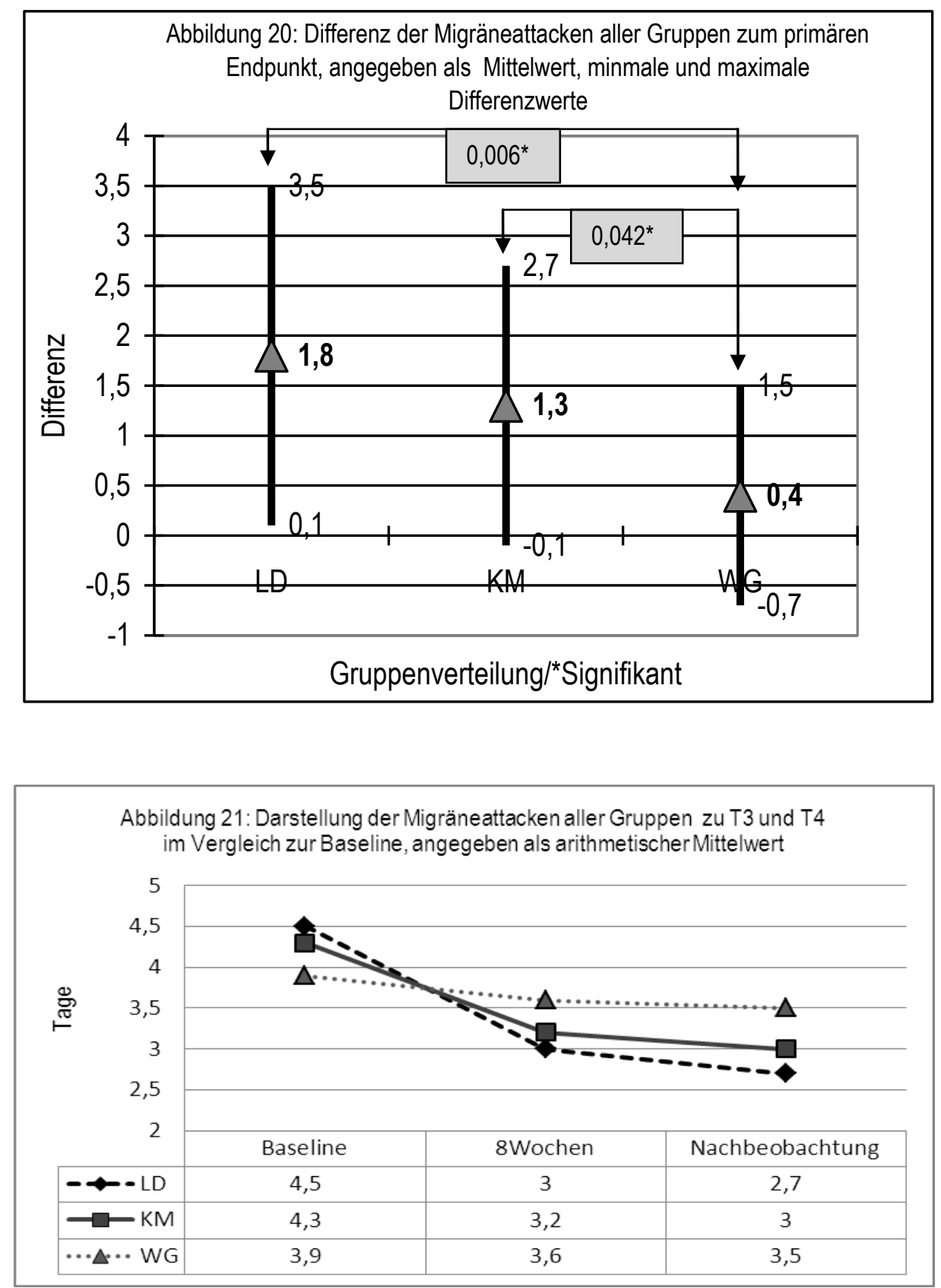


\begin{tabular}{|c|c|c|c|}
\hline & Lymphdrainage & Klassische Massage & Wartegruppe \\
\hline Baseline & $8,8(5,2)$ & $6,7(5,6)$ & $6,6(3,1)$ \\
\hline 4 Wochen & $6,9(4,6)$ & $4,7(3,7)$ & $7,2(5,3)$ \\
\hline 8 Wochen & $5,4(4,4)$ & $4,5(3,2)$ & $7,1(4,2)$ \\
\hline Nachbeobachtung & $5,3(4,0)$ & $4,6(4,0)$ & $7,2(4,6)$ \\
\hline
\end{tabular}

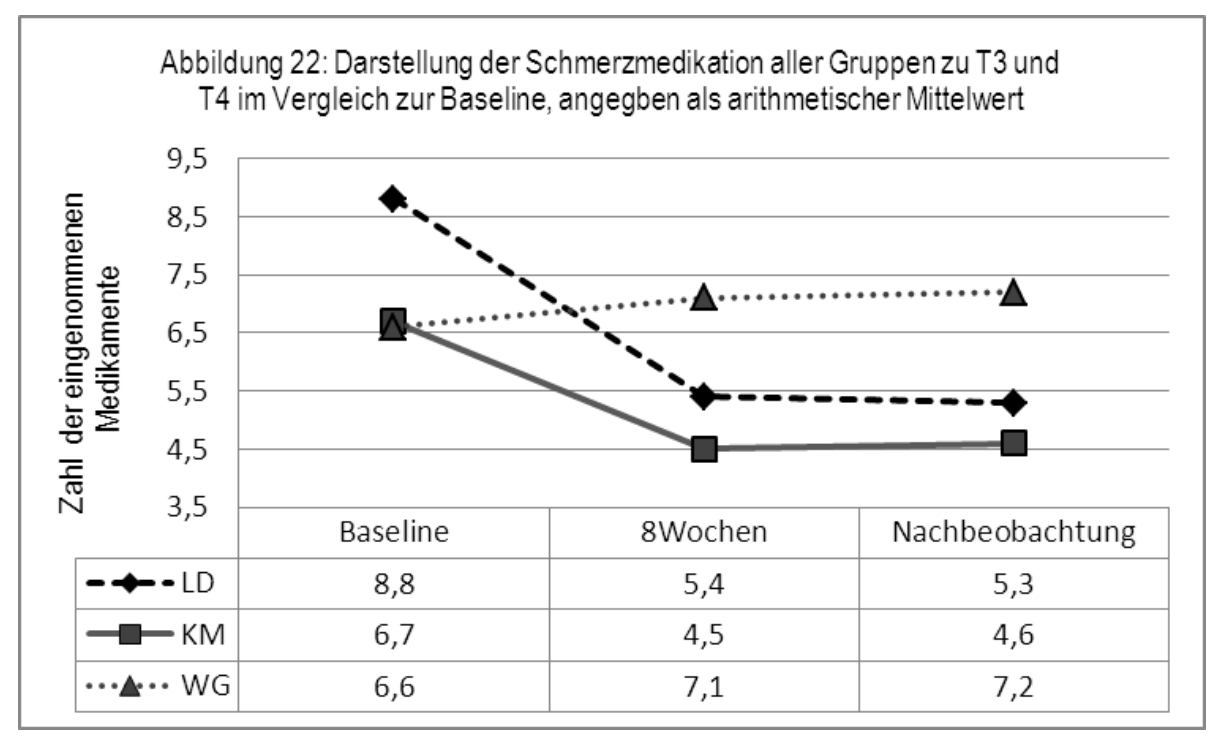

Zum Beobachtungsende ("T4=P") finden sich keine signifikanten Unterschiede der Responderraten bzgl. Migräneattacken $(p=0,326)$ und Migränetage $(p=0,126)$. Bei der Differenz in den Migräneattacken $(p=0,016)$ und den Migränetagen $(p=0,019)$ findet sich ein signifikanter Unterschied zwischen der LDund der WG-Gruppe ( $p=0,006$ bzw. $p=0,015)$ sowie zwischen der KM- und der WG-Gruppe $(p=0,042$ bzw. $p=0,016$ ) (s. Tabelle 34) (s. Abbildung 20). Im Verlauf zeigt sich eine signifikante Abnahme der Zahl der eingenommenen Akutmedikamente in der LD-Gruppe im Vergleich zu den beiden anderen Gruppen ( $p=0,004)$ (s. Tabelle 33). 


\begin{tabular}{|c|c|c|c|c|}
\hline & Lymphdrainage & Klassische Massage & Wartegruppe & $p$-Wert \\
\hline $\begin{array}{l}\text { Responder } \\
\text { Migräneattacken, \% }\end{array}$ & 38 & 33 & 18 & $0,326^{c}$ \\
\hline $\begin{array}{l}\text { Responder } \\
\text { Migränetage, \% }\end{array}$ & 38 & 38 & 14 & $0,126^{c}$ \\
\hline $\begin{array}{l}\text { Differenz } \\
\text { Migräneattacken, } \mathrm{n}\end{array}$ & $1,8(1,7)$ & $1,3(1,4)$ & $0,4(1,1)$ & $0,016^{a}$ \\
\hline $\begin{array}{l}\text { Differenz } \\
\text { Migränetage, } n\end{array}$ & $3,1(4,4)$ & $2,4(3,9)$ & $0,2(2,5)$ & $0,019 b$ \\
\hline $\begin{array}{l}\text { Differenz } \\
\text { Kopfschmerztage } \\
\end{array}$ & $3,1(4,8)$ & $2,8(3,5)$ & $0,5(2,6)$ & 0,064 \\
\hline \multicolumn{5}{|c|}{$\begin{array}{l}\text { a } L D \text { versus KM } p=0,419 ; \text { LD versus WG } p=0,006 ; K M \text { versus WG } p=0,042 \\
\text { b } L D \text { versus KM } p=0,869 ; \text { LD versus WG } p=0,015 ; K M \text { versus WG } p=0,016 \\
\text { c post-hoc Tests nicht signifikant }\end{array}$} \\
\hline
\end{tabular}

\subsubsection{Zwischen Baseline und Behandlungsende (nach 8 Wochen; sekundäre Endpunkte)}

In diesem Abschnitt werden die Responderraten (mind. 50\%ige Reduktion der Migräneattacken und Migränetage) und die Differenz der Migräneattacken (MA), Migränetage (MT), Kopfschmerztage (KSTage) sowie die Differenz in der Einnahme von akuten Schmerzmedikamenten (SM) zwischen Baseline „T1" und dem Ende der Behandlung "T3=8" aller drei Gruppen als sekundäre Endpunkte verglichen.

Zum Ende der Behandlung "T3=8“ findet sich außer bei den Migränetagen kein signifikanter Unterschied in den Responderraten in allen Gruppen. Der Unterschied der Responderrate der Migränetage ist zwischen der LD-Gruppe und der WG-Gruppe signifikant $(p=0,033)$, nicht aber zwischen der KM- und der WG-Gruppe. Bei die Differenz der Migräneattacken findet sich ein signifikanter Unterschied zwischen der LD- und der WG-Gruppe $(p=0,013)$ und zwischen der KM- und der WG-Gruppe $(0,033)$ (s. Abbildung 23), jedoch nicht zwischen der LD- und der KM-Gruppe (s. Tabelle 35). Im Verlauf zeigt sich eine signifikante Abnahme der Zahl der eingenommenen Akutmedikamente in der LD-Gruppe im Vergleich zu den beiden anderen Gruppen $(p=0,004)$. 


\begin{tabular}{|c|c|c|c|c|}
\hline & Lymphdrainage & Klassische Massage & Wartegruppe & p-Wert \\
\hline $\begin{array}{l}\text { Responder } \\
\text { Migräneattacken, \% }\end{array}$ & 24 & 29 & 14 & 0,479 \\
\hline $\begin{array}{l}\text { Responder } \\
\text { Migränetage, \% }\end{array}$ & 29 & 14 & 5 & $0,093^{a}$ \\
\hline $\begin{array}{l}\text { Differenz } \\
\text { Migräneattacken, } n\end{array}$ & $1,5(1,7)$ & $1,1(1,3)$ & $0,3(1,1)$ & $0,025^{b}$ \\
\hline $\begin{array}{l}\text { Differenz } \\
\text { Migränetage, } n\end{array}$ & $1,6(4,2)$ & $1,4(3,5)$ & $0,6(1,6)$ & 0,162 \\
\hline $\begin{array}{l}\text { Differenz } \\
\text { Kopfschmerztage, } n\end{array}$ & $2,8(4,4)$ & $1,3(3,2)$ & $0,8(1,7)$ & 0,290 \\
\hline
\end{tabular}

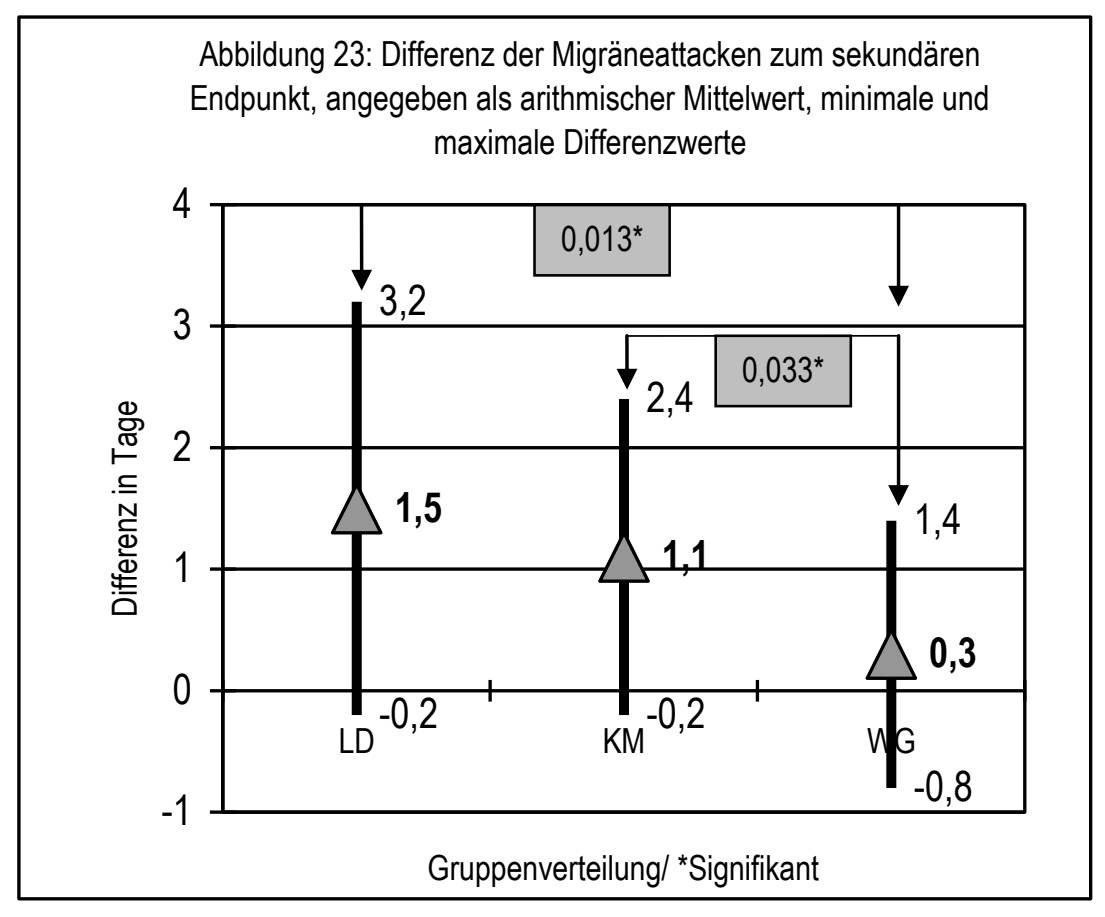




\subsection{Begleitsymptome und Schmerzintensität aller Gruppen im Vergleich}

\subsubsection{Zur Nachbeobachtung}

Zum Zeitpunkt Nachbeobachtung („T4“) zeigen die Lymphdrainage und die klassische Massage eine signifikante Reduzierung der Schmerzintensität im Vergleich zur Wartegruppe (s. Tabelle 36 und Abbildung 24). Im Verlauf zeigt sich sowohl in der LD- als auch in der KM-Gruppe eine signifikante Reduktion der Photophobie (s. Tabellen 19 und 23), in der LD-Gruppe zusätzlich auch der Schmerzintensität (s. Tabelle 19).

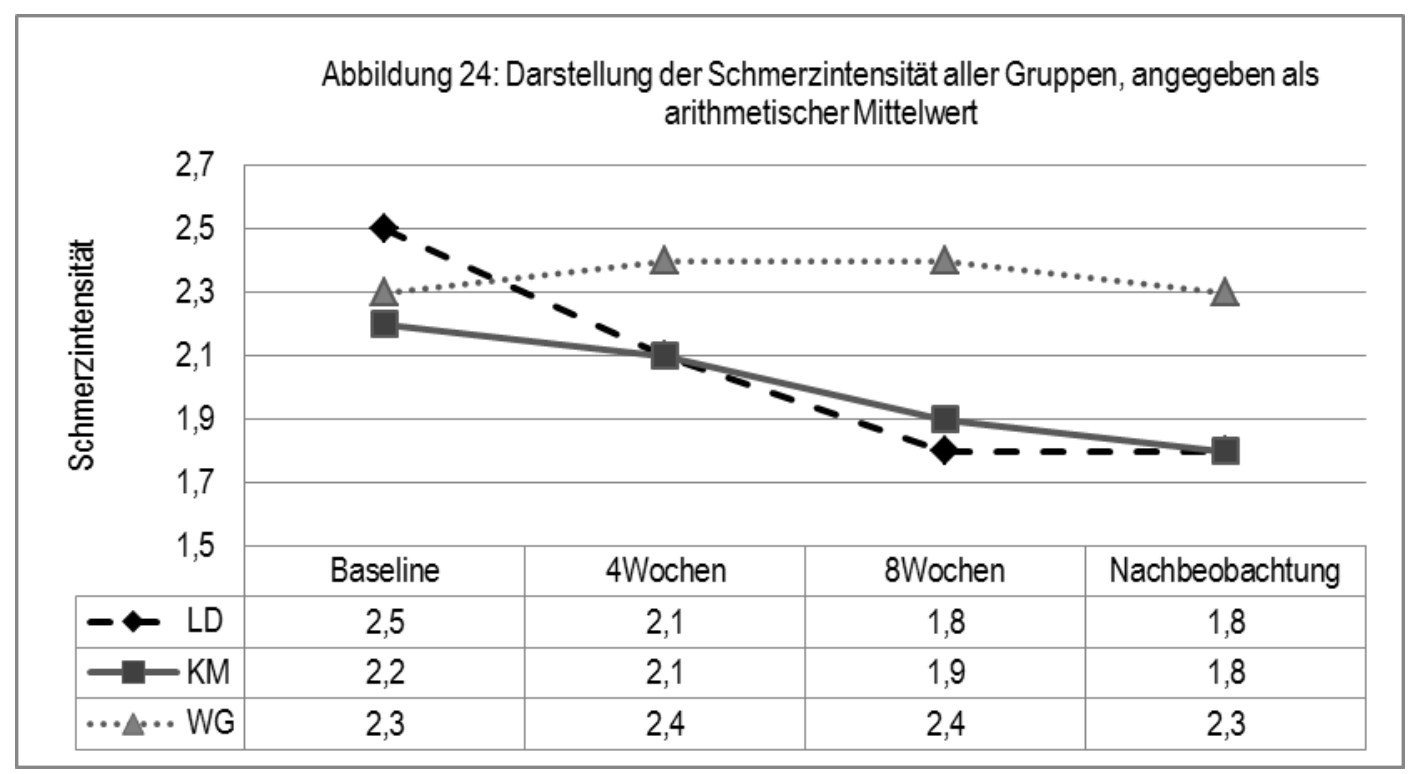

\begin{tabular}{|l|l|l|l|l|}
\hline $\begin{array}{l}\text { Tabelle 36: Schmerzintensität aller Gruppen zu allen Zeitpunkten, angegeben als arithmetischer } \\
\text { Mittelwert (Standardabweichung in Klammern) }\end{array}$ \\
\hline & Lymphdrainage & Klassische Massage & Wartegruppe & p-Wert \\
\hline Baseline & $2,5(0,5)$ & $2,2(0,5)$ & $2,3(0,5)$ & 0,135 \\
\hline 4 Wochen & $2,1(0,7)$ & $2,1(1,0)$ & $2,4(1,0)$ & 0,529 \\
\hline 8 Wochen & $1,8(0,9)$ & $1,9(0,6)$ & $2,4(0,7)$ & $\mathbf{0 , 0 1 1}$ \\
\hline Nachbeobachtung & $1,8(0,9)$ & $1,8(0,9)$ & $2,3(0,6)$ & $\mathbf{0 , 0 2 6}$ \\
\hline
\end{tabular}

\subsubsection{Zum Behandlungsende}

Zum Behandlungsende („T3“) zeigen die Lymphdrainage und die klassische Massage eine signifikante Reduzierung der Schmerzintensität im Vergleich zur Wartegruppe (s. Tabelle 36 und Abbildung 24). Im Verlauf zeigt sich sowohl in der LD- als auch in der KM-Gruppe eine signifikante Reduktion der Photophobie (s. Tabellen 19 und 23), in der LD-Gruppe zusätzlich auch der Schmerzintensität (s. Tabelle 19). 


\subsection{Veränderung der psychometrischen Parameter aller Gruppen im Vergleich}

\subsubsection{Beeinträchtigung durch Kopfschmerzen (IBK)}

Bezüglich der akuten Beeinträchtigung durch Kopfschmerzen findet sich in beiden Behandlungsgruppen (LD und KM), aber auch in der Wartegruppe (WG) ein signifikanter Rückgang des Gesamtscores des IBK im Verlauf (s. Tabellen 20, 24, 28 und 37 sowie Abbildung 25 und 26). Die akute Beeinträchtigung durch Kopfschmerzen geht somit sowohl in den beiden Behandlungsgruppen als auch in der Wartegruppe zurück.

\begin{tabular}{|l|l|l|l|l|}
\hline \multicolumn{5}{|l}{$\begin{array}{l}\text { Tabelle 37: Ergebnisse des IBK im Verlauf der Untersuchung in allen drei Gruppen, angegeben als } \\
\text { arithmetischer Mittelwert (Standardabweichung in Klammern) }\end{array}$} \\
\hline & Lymphdrainage & Klassische Massage & Wartegruppe & p-Wert \\
\hline Baseline & $64,2(12,9)$ & $58,7(19,8)$ & $54,7(16,8)$ & 0,101 \\
\hline 4 Wochen & $51,1(20,4)$ & $54,5(22,7)$ & $51,4(16,6)$ & 0,671 \\
\hline 8 Wochen & $49,5(19,3)$ & $49,5(20,8)$ & $45,3(13,7)$ & 0,554 \\
\hline Nachbeobachtung & $49,9(24,7)$ & $48,2(22,6)$ & $42,0(20,4)$ & 0,498 \\
\hline
\end{tabular}

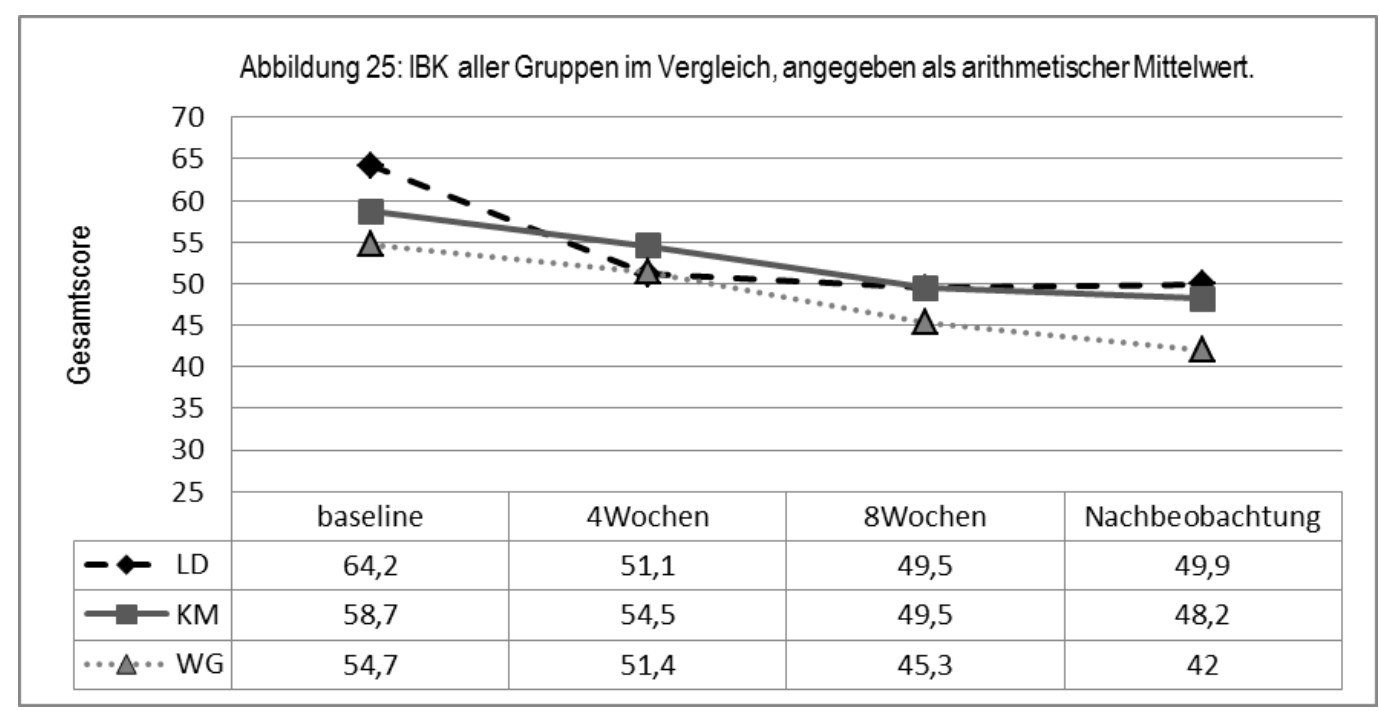




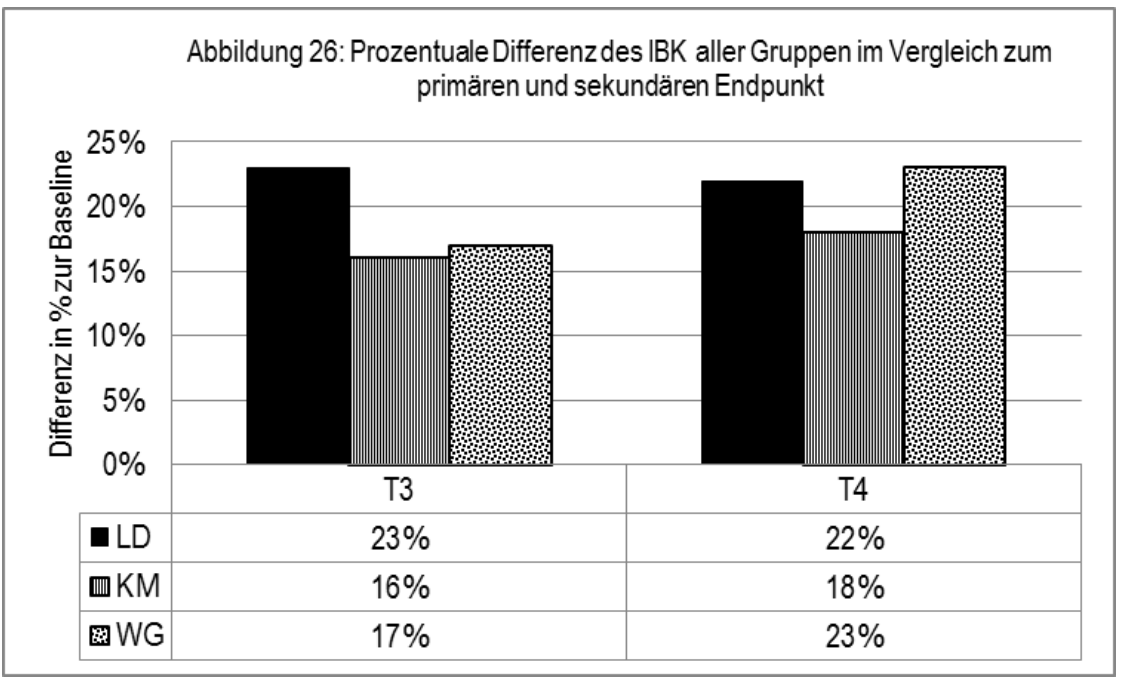

\subsubsection{Einschränkung der Leistungsfähigkeit (Midas)}

Bei der Einschränkung der Leistungsfähigkeit (gemessen mit Midas) findet sich in der LD-Gruppe eine signifikante Abnahme im Verlauf (s. Tabelle 20), nicht jedoch in der KM- und in der WG-Gruppe (s. Tabellen 24, 28 und 38, Abbildung 27). Zwischen den Gruppen findet sich zu keinem Zeitpunkt ein signifikanter Unterschied (s. Tabellen 38 und 39, Abbildung 28).

Die Lymphdrainage verbessert die Leistungsfähigkeit bzw. reduziert die verlorenen Tage wegen Migräne signifikant in Vergleich zur KM- oder zur WG-Gruppe.

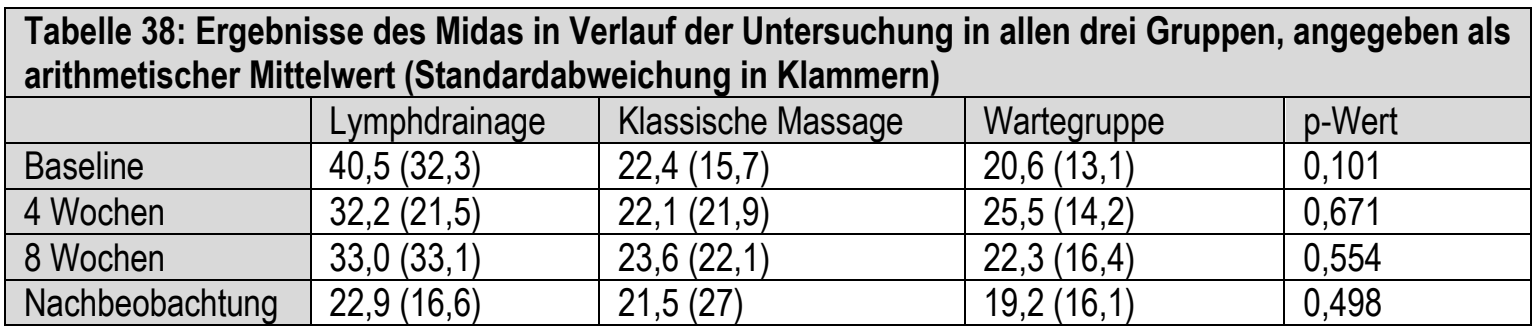

\begin{tabular}{|c|c|c|c|}
\hline \multicolumn{4}{|c|}{$\begin{array}{l}\text { Tabelle 39: Unterschied der Differenz des Midas in allen drei Gruppen zwischen } \\
\text { Baseline und Behandlungsende (sekundärer Endpunkt) sowie zwischen Baseline und } \\
\text { Nachbeobachtung (primärer Endpunkt). Angaben im arithmetischen Mittelwert } \\
\text { (Standardabweichung in Klammern). }\end{array}$} \\
\hline & Lymphdrainage & Klassische Massage & Wartegruppe \\
\hline $\begin{array}{l}\text { Zur sekundären } \\
\text { Endpunkt }\end{array}$ & $-7,7(19,7)$ & $0,5(19,4)$ & $2,4(12,1)$ \\
\hline $\begin{array}{l}\text { Zur primären } \\
\text { Endpunkt }\end{array}$ & $-11,2(16,1)$ & $-1,6(23,0)$ & $0,4(12,1)$ \\
\hline
\end{tabular}



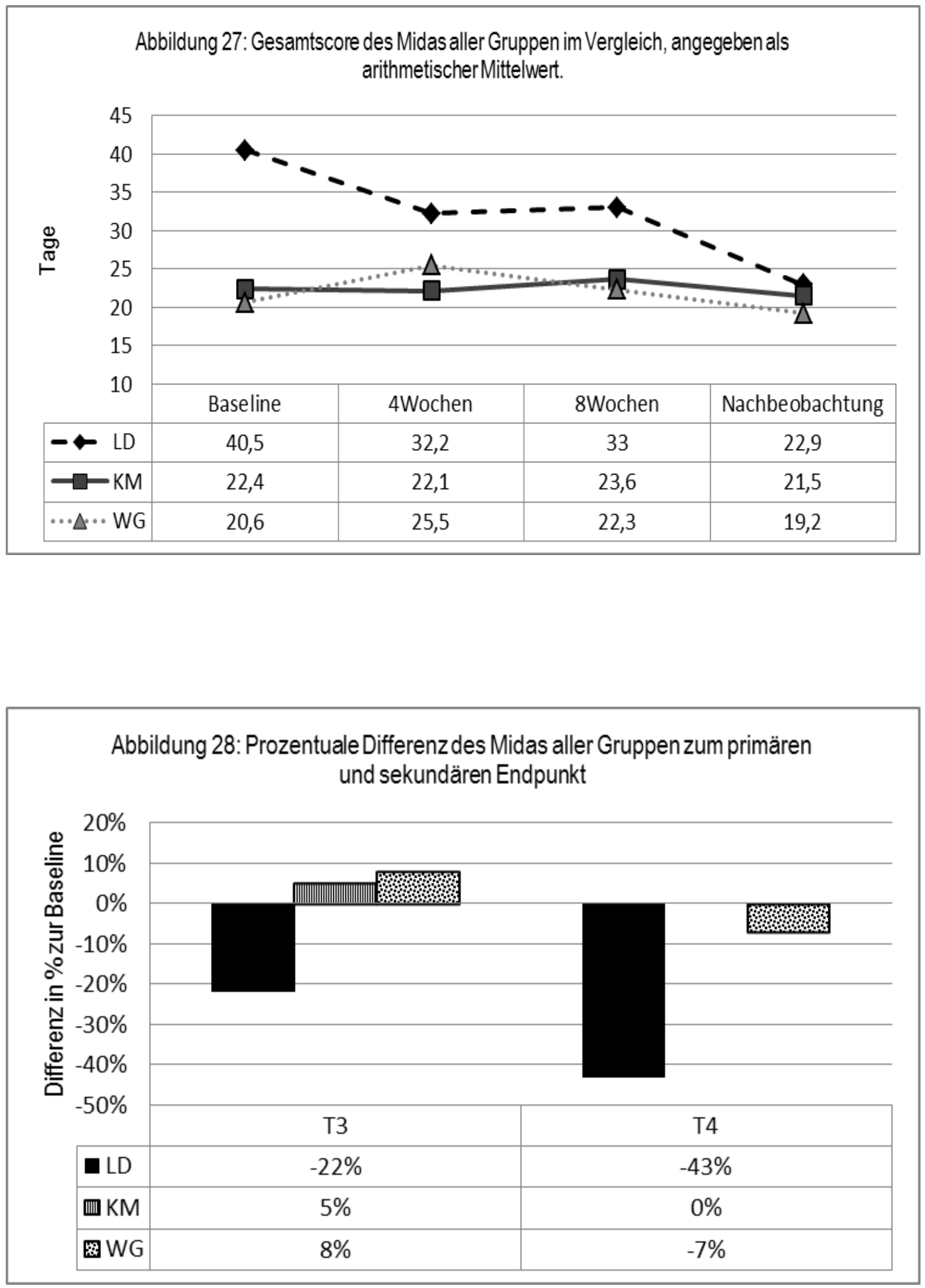


\subsubsection{Depressive Symptome (CES-D)}

Depressive Symptome nehmen im Verlauf nur in der KM-Gruppe signifikant ab (s. Tabelle 24), nicht jedoch in der LD-Gruppe (s. Tabelle 20) und in der WG-Gruppe (s. Tabelle 28). Zum Behandlungsende und bei der Nachbeobachtung zeigen sich signifikante Unterschiede zwischen den Gruppen (Tabelle 40, Abbildung 29 und 30).

\begin{tabular}{|l|l|l|l|l|}
\hline \multicolumn{5}{|l|}{$\begin{array}{l}\text { Tabelle 40: Ergebnisse der CES-D im Verlauf der Untersuchung in allen drei Gruppen, } \\
\text { angegeben als arithmetischer Mittelwert }\end{array}$} \\
\hline & Lymphdrandardabweichung in Klammern) & Klassische Massage & Wartegruppe & p-Wert \\
\hline Baseline & $14,9(8,3)$ & $14,0(8,4)$ & $15,1(7,7)$ & 0,843 \\
\hline 4 Wochen & $12,0(6,5)$ & $12,8(7,3)$ & $14,4(8,3)$ & 0,747 \\
\hline 8 Wochen & $9,6(6,8)$ & $12,0(7,8)$ & $15,8(8,1)$ & $\mathbf{0 , 0 3 2}$ \\
\hline Nachbeobachtung & $11,2(10)$ & $12,1(9,0)$ & $16,7(9,8)$ & $\mathbf{0 , 0 4 3}$ \\
\hline
\end{tabular}
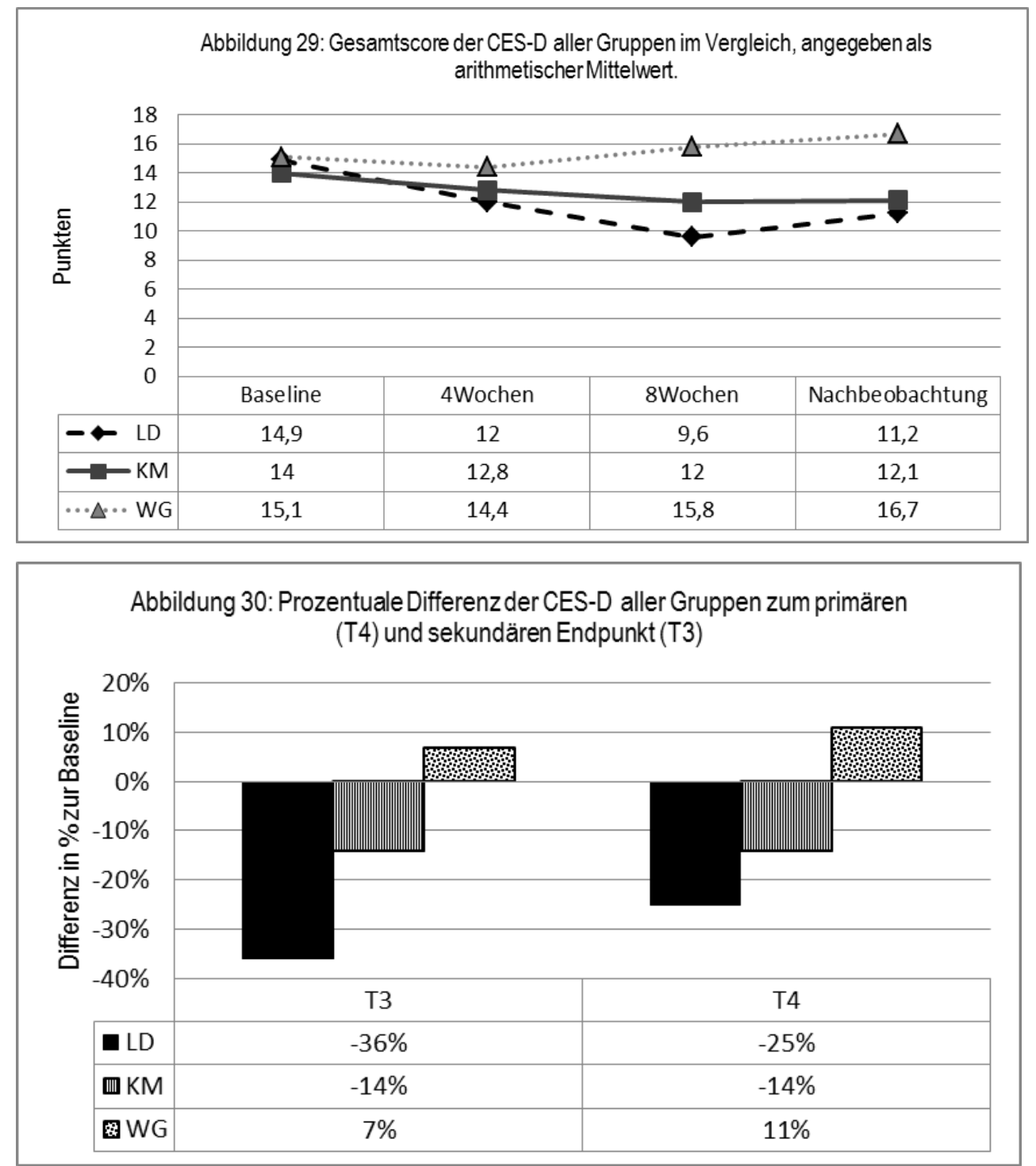


\subsubsection{Lebensqualität (SF-36)}

In der körperlichen Summenskala findet sich eine signifikante Zunahme der Lebensqualität im Verlauf in allen drei Gruppen, ohne signifikanten Unterschied zwischen den Gruppen (s. Tabellen 20, 24, 28 und 41, Abbildungen 31 und 33)

\begin{tabular}{|l|l|l|l|l|}
\hline \multicolumn{5}{|l|}{$\begin{array}{l}\text { Tabelle 41: Ergebnisse des SF-36 im Verlauf der Untersuchung in allen drei Gruppen (körperliche } \\
\text { Summenskala), angegeben in transformierten Werten }\end{array}$} \\
\hline & Lymphdrainage & Klassische Massage & Wartegruppe & p-Wert \\
\hline Baseline & 41,9 & 42,3 & 41,4 & 0,560 \\
\hline 4 Wochen & 43,4 & 41,0 & 39,9 & 0,067 \\
\hline 8 Wochen & 45,1 & 43,6 & 44,1 & 0,155 \\
\hline Nachbeobachtung & 44,0 & 45,4 & 43,6 & 0,098 \\
\hline
\end{tabular}

Bei der psychischen Summenskala zeigt sich nur in der KM-Gruppe eine signifikante Zunahme der Lebensqualität im Verlauf. Zwischen den Gruppen zeigt sich nur zum Behandlungsende ein signifikanter Unterschied (s. Tabellen 20, 24, 28 und 42, Abbildungen 32 und 33).

\begin{tabular}{|l|l|l|l|l|}
\hline \multicolumn{5}{|l|}{$\begin{array}{l}\text { Tabelle 42: Ergebnisse des SF-36 im Verlauf der Untersuchung in allen drei Gruppen (psychische } \\
\text { Summenskala), angegeben in transformierten Werten }\end{array}$} \\
\hline & Lymphdrainage & Klassische Massage & Wartegruppe & p-Wert \\
\hline Baseline & 42,3 & 46,7 & 44,5 & 0,467 \\
\hline 4 Wochen & 49,1 & 50,7 & 45,8 & 0,118 \\
\hline 8 Wochen & 48,4 & 50,0 & 42,1 & 0,021 \\
\hline Nachbeobachtung & 49,2 & 48,9 & 45,0 & 0,102 \\
\hline
\end{tabular}

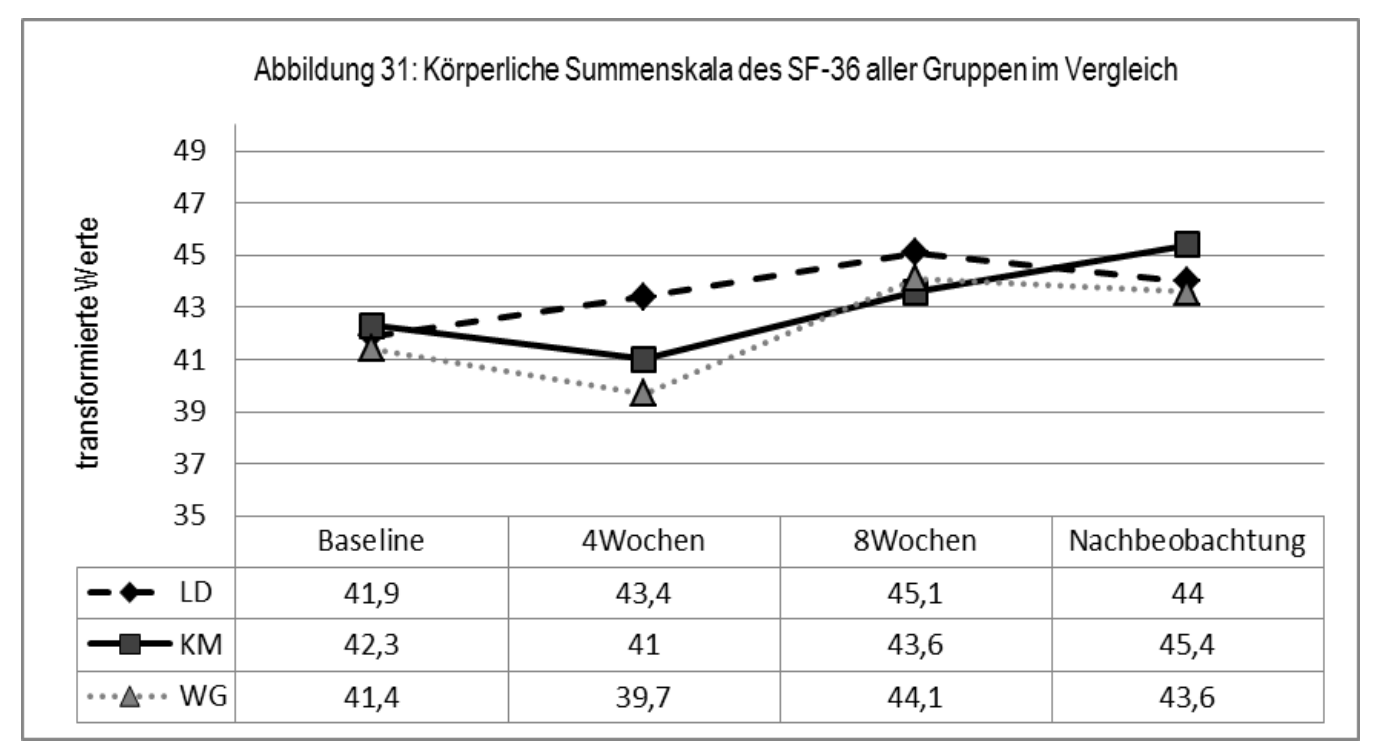




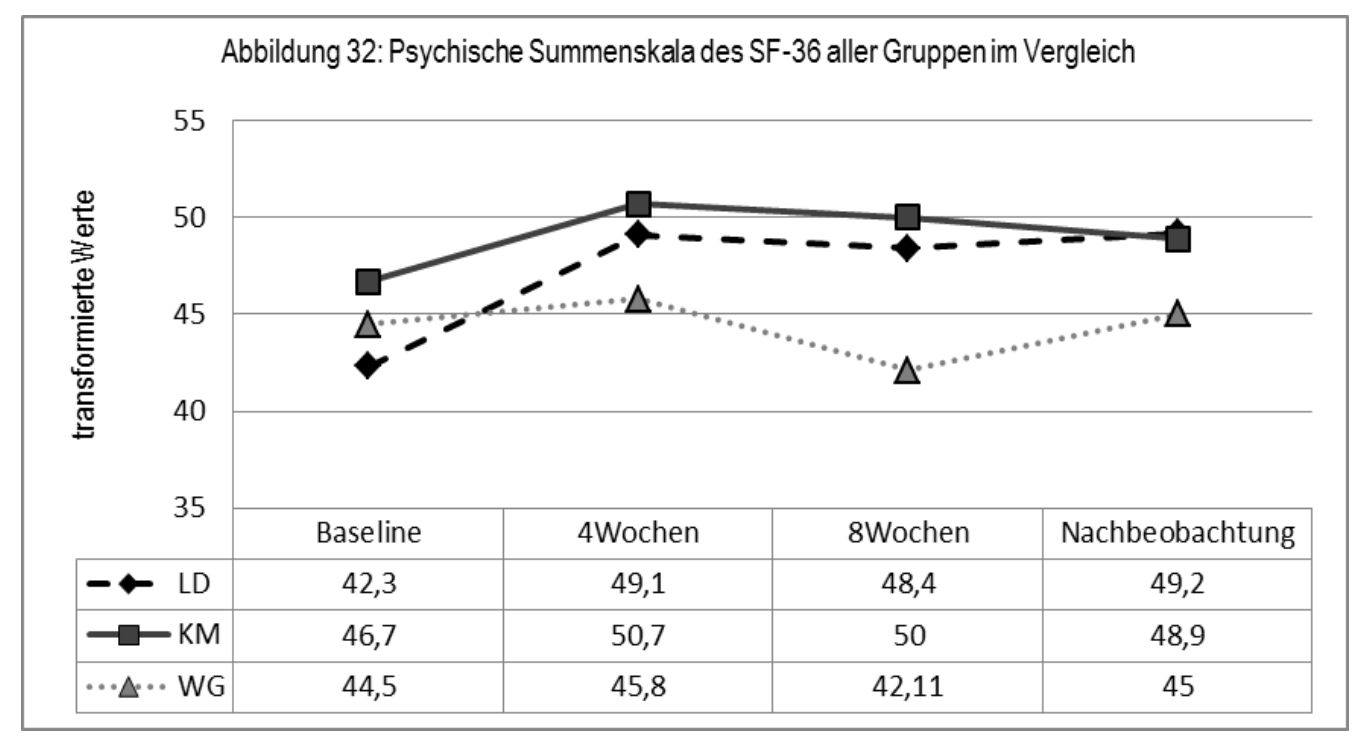

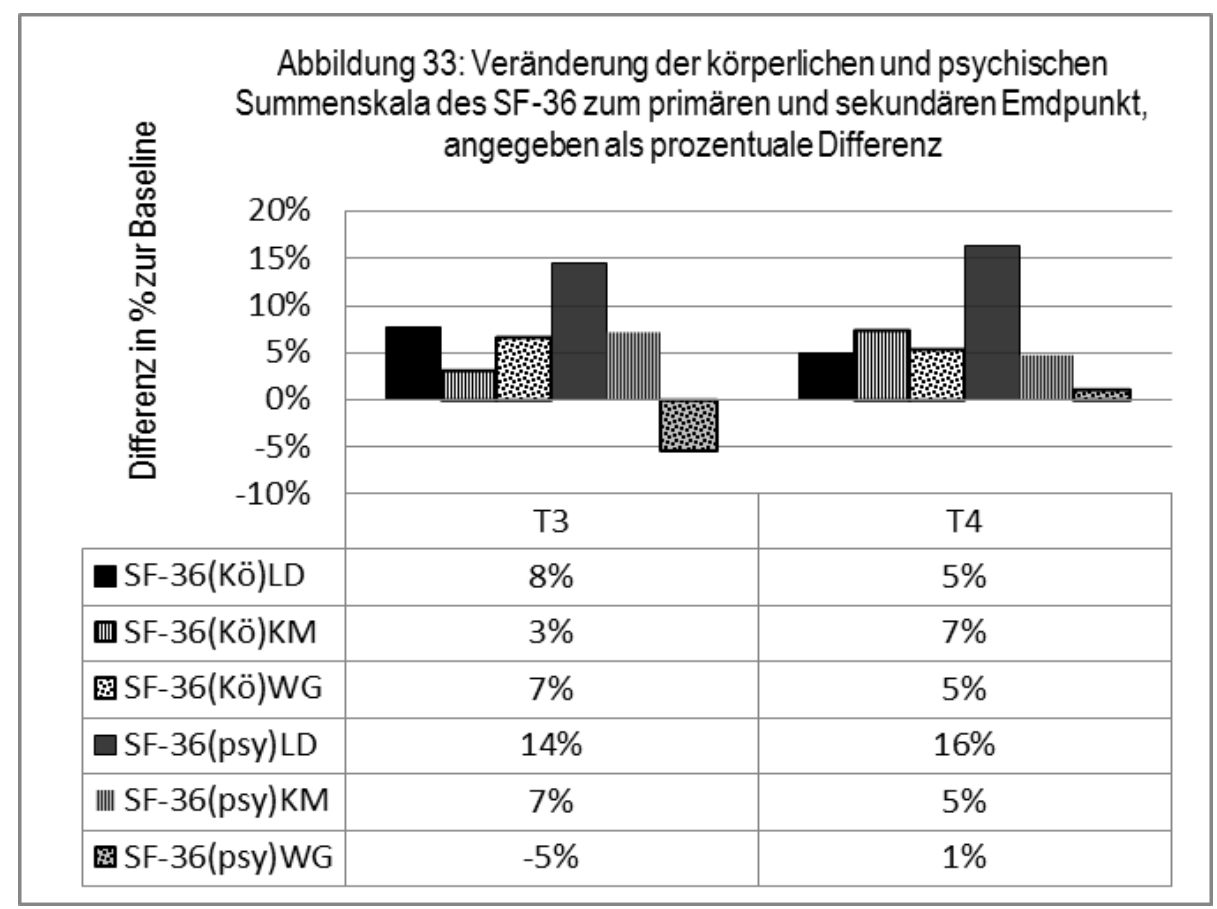


Bei den Subskalen des SF-36 finden sich eine signifikante Zunahme in der körperlichen Rollenfunktion in allen drei Gruppen, bei den körperlichen Schmerzen in der LD-Gruppe und in der WG-Gruppe, in der körperlichen Funktionsfähigkeit und der Vitalität in der KM-Gruppe sowie in der sozialen Funktionsfähigkeit in der LD-Gruppe und der KM-Gruppe (s. Tabellen 21, 25 und 29).

Zwischen den Gruppen findet sich ein signifikanter Unterschied in der körperlichen Rollenfunktion und der sozialen Funktionsfähigkeit zum Zeitpunkt der Nachbeobachtung sowie in der Vitalität zum Behandlungsende (Tabellen 43 und 44, Abbildungen 34 und 35)

\begin{tabular}{|c|c|c|c|c|}
\hline \multicolumn{5}{|c|}{$\begin{array}{l}\text { Tabelle 43: Ergebnisse aller Subskalen des SF-36 in allen drei Gruppen zum Zeitpunkt } \\
\text { der Nachbeobachtung (T4), angegeben als arithmetischer Mittelwert } \\
\text { (Standardabweichung in Klammern) }\end{array}$} \\
\hline & Lymphdrainage & Klassische Massage & Wartegruppe & $p$-Wert \\
\hline KÖFU & $86,1(11,8)$ & $88,2(13,8)$ & $87,4(10,8)$ & 0,248 \\
\hline KÖRO & $68,1(35,2)$ & $76,3(36,8)$ & $58,2(29,4)$ & 0,047 \\
\hline SCHM & $47,8(28,0)$ & $50,2(26,1)$ & $44,0(12,2)$ & 0,510 \\
\hline AGES & $60,8(15,4)$ & $60,4(20,1)$ & $57,5(15,3)$ & 0,605 \\
\hline VITA & $57,5(14,5)$ & $57,4(19,7)$ & $52,1(12,5)$ & 0,314 \\
\hline SOFU & $77,1(19,8)$ & $84,2(19,0)$ & $71,4(16,5)$ & 0,027 \\
\hline EMRO & $85,2(26,1)$ & $80,7(32,0)$ & $66,7(39,3)$ & 0,108 \\
\hline PSYC & $68,7(18,4)$ & $68,8(20,3)$ & $65,2(18,4)$ & 0,425 \\
\hline \multicolumn{5}{|c|}{$\begin{array}{l}\text { KÖFU: Körperliche Funktionsfähigkeit, KÖRO: Körperliche Rollenfunktion, SCHM: Körperliche } \\
\text { Schmerzen, AGES: Allgemeine Gesundheitswahrnehmung, VITA: Vitalität, SOFU: Soziale } \\
\text { Funktionsfähigkeit, EMRO: Emotionale Rollenfunktion, PSYC: Psychisches Wohlbefinden. }\end{array}$} \\
\hline
\end{tabular}

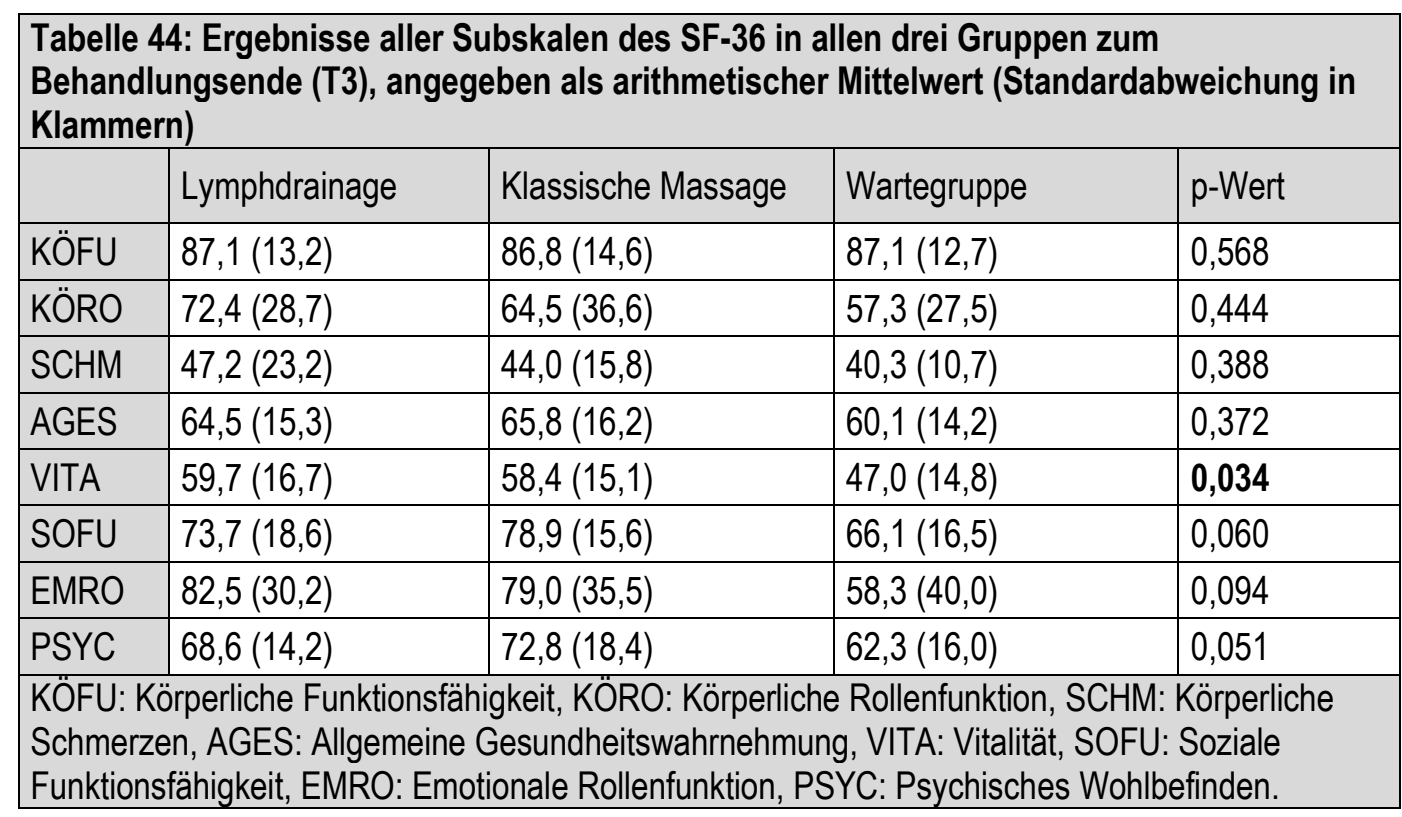


Abbildung 34: Alle Subskalen des SF-36 aller Gruppen zum primären

Endpunkt, angegeben als prozentuale Differenz zur Baseline
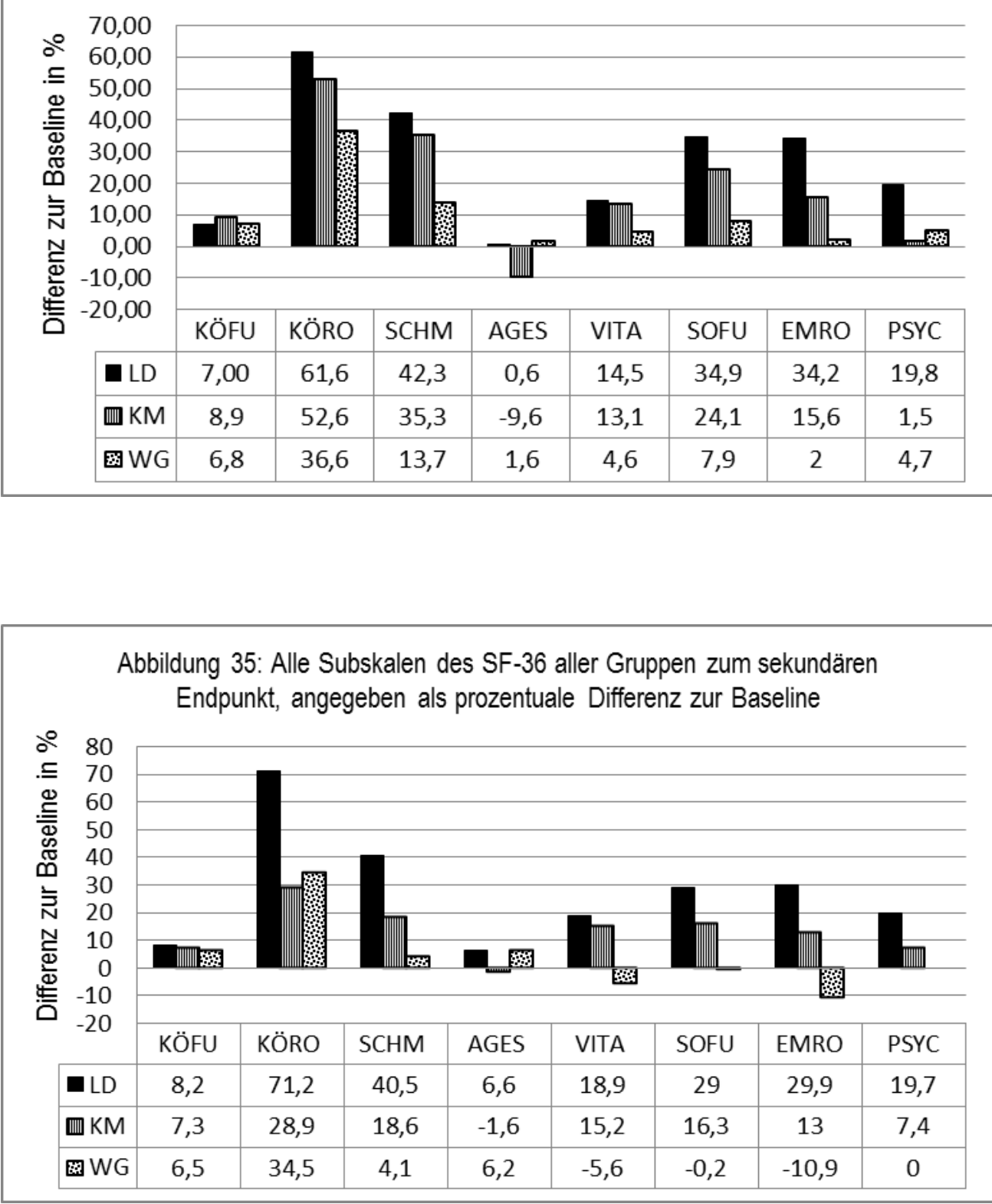


\subsubsection{Schlafqualität (PSQI)}

Die Schlafqualität ist zu Beginn der Untersuchung in allen drei Gruppen gleichermaßen beeinträchtigt (oberhalb des Normwertes von 5). Bei allen drei Gruppen findet sich ein niedrigerer Gesamtscore des PSQI im Verlauf, dieser ist jedoch nur in der KM-Gruppe signifikant (s. Tabellen 20, 24 und 28). Zwischen den Gruppen findet sich kein signifikanter Unterschied des PSQI (s. Tabelle 45, Abbildung 36 und 37).

\begin{tabular}{|c|c|c|c|c|}
\hline & Lymphdrainage & Klassische Massage & Wartegruppe & $p$-Wert \\
\hline Baseline & $6,7(2,9)$ & $6,3(3,2)$ & $7,3(3,3)$ & 0,777 \\
\hline 4 Wochen & $5,5(3,0)$ & $5,9(2,5)$ & $7,2(3,1)$ & 0,184 \\
\hline 8 Wochen & $4,7(2,3)$ & $4,7(2,2)$ & $6,5(3,0)$ & 0,052 \\
\hline Nachbeobachtung & $4,9(2,3)$ & $5,6(3,0)$ & $6,3(3,6)$ & 0,167 \\
\hline
\end{tabular}

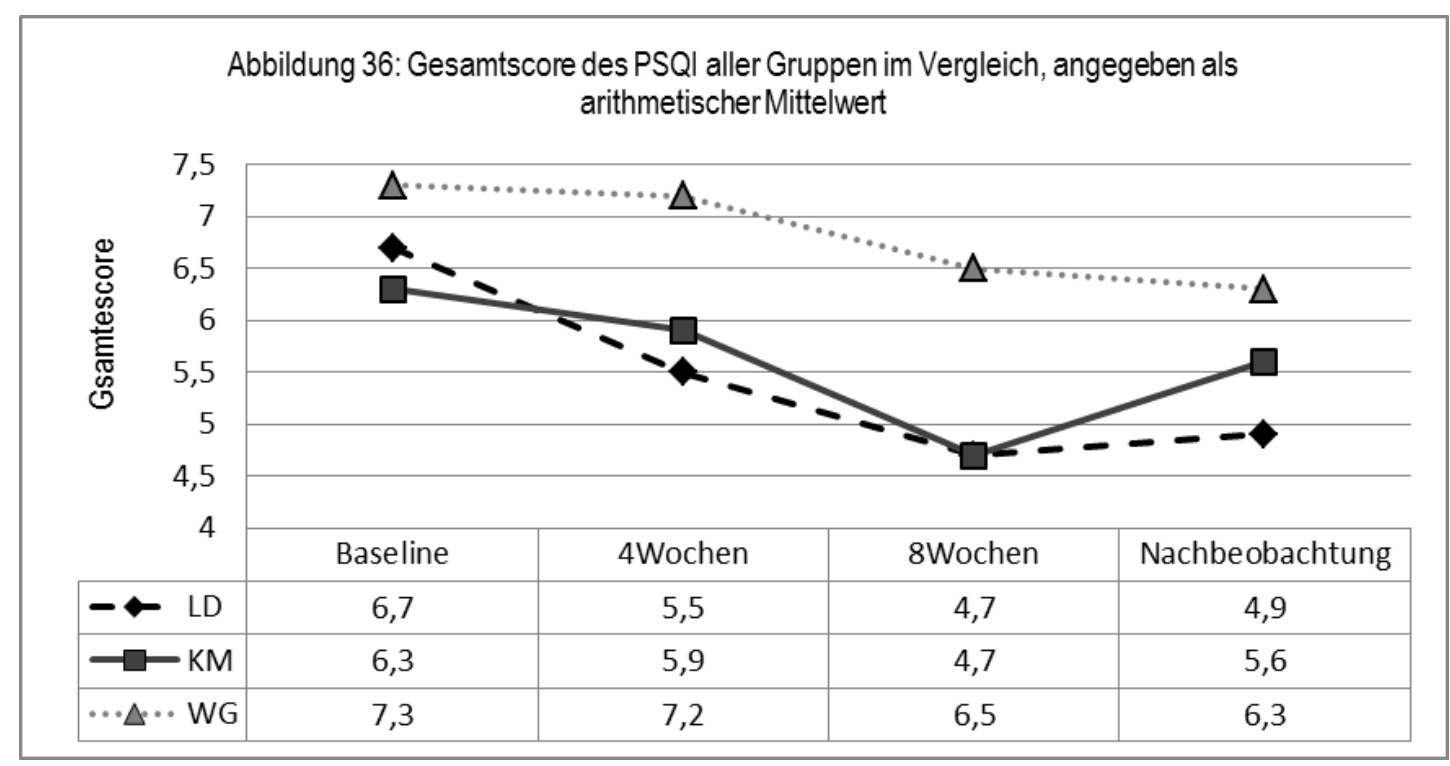




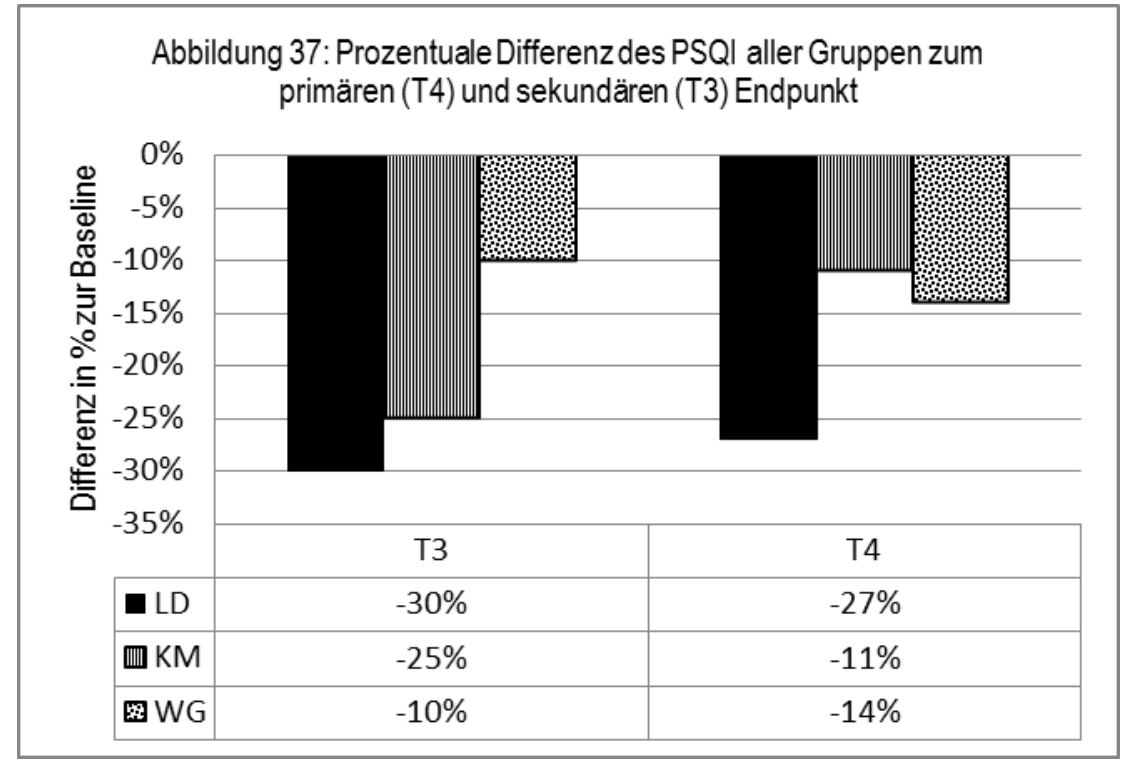




\subsubsection{Objektive Symptomschwere (CGI)}

Es findet sich sowohl in der LD- als auch in der KM-Gruppe eine signifikante Verbesserung des CGI im Verlauf (s. Tabellen 20 und 24). In der WG ist keine Veränderung des CGI im Verlauf zu beobachten (s. Tabelle 28). Ein signifikanter Unterschied zwischen den Gruppen zeigt der CGI zum Behandlungsende und zum Zeitpunkt der Nachbeobachtung (s. Tabelle 46, Abbildung 38). Bei dem Unterschied in der Differenz des CGI in der LD-Gruppe (-50\% zu T3 und $-45 \%$ zu T4) findet sich zu beiden Endpunkten eine deutliche Besserung der objektiven Symptomschwere, ebenfalls in der KM-Gruppe (-33\% und $-39 \%$ ), nicht jedoch in der WG-Gruppe (jeweils $-5 \%$ ) (s. Abbildung 39 ).

\begin{tabular}{|c|c|c|c|c|}
\hline & Lymphdrainage & Klassische Massage & Wartegruppe & $\mathrm{p}$-Wert \\
\hline Baseline & $2,0(0,9)$ & $1,8(0,6)$ & $1,9(0,5)$ & 0,684 \\
\hline 4 Wochen & $1,4(0,5)$ & $1,7(0,7)$ & $1,8(0,6)$ & 0,103 \\
\hline 8 Wochen & $1,0(0,2)$ & $1,2(0,4)$ & $1,8(0,4)$ & 0,001 \\
\hline Nachbeobachtung & $1,1(0,5)$ & $1,1(0,3)$ & $1,8(0,5)$ & 0,001 \\
\hline
\end{tabular}

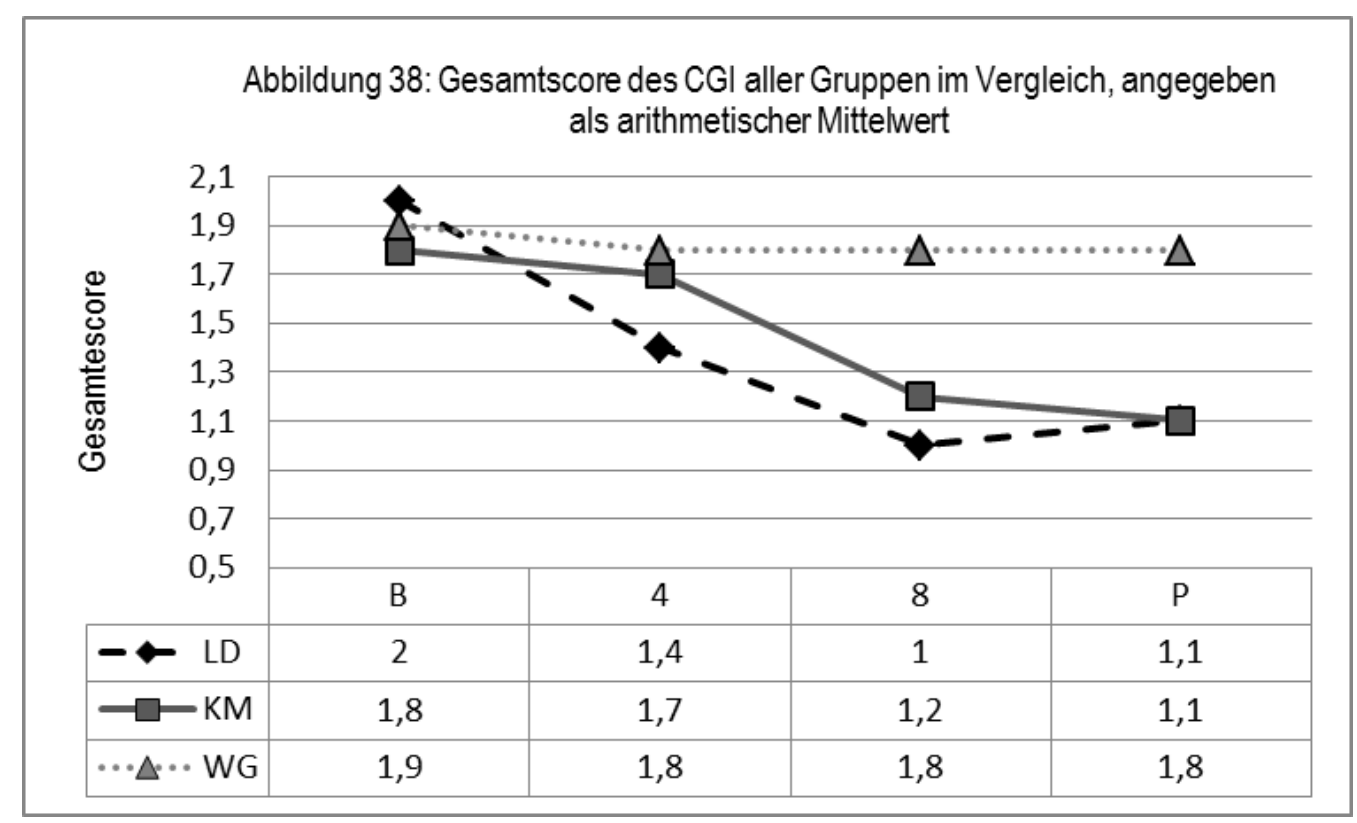




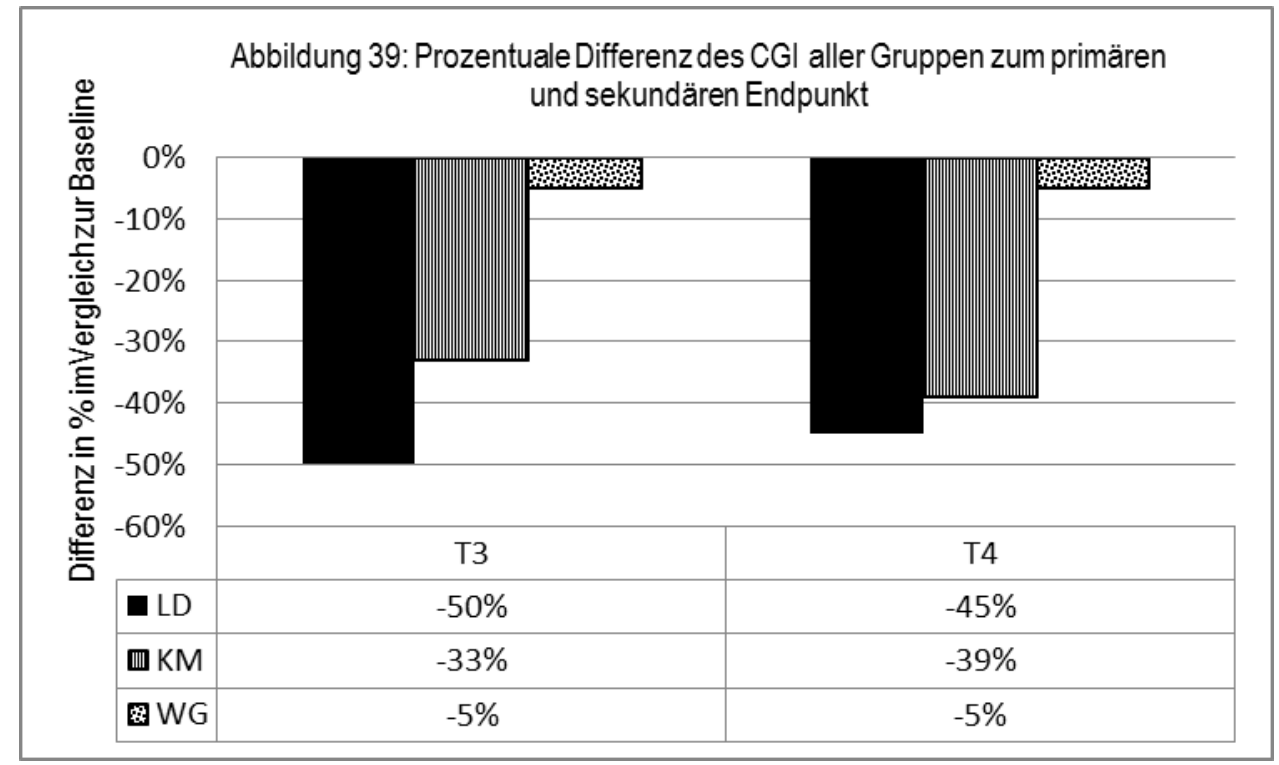




\section{Diskussion}

\subsection{Diskussion der Studienergebnisse}

Es gibt bisher keine randomisierten kontrollierten Studien zur Untersuchung der Wirksamkeit von Lymphdrainage in der Migräneprophylaxe, dementsprechend handelt es sich bei der vorliegenden Arbeit um die erste kontrollierte randomisierte Studie zur Untersuchung der Wirksamkeit der Lymphdrainage im Vergleich zur klassischen Massage bei Migränepatienten.

In dieser Studie führten beide Physikalischen Therapiemaßnahmen KM und LD zu einer signifikanten Reduktion der Migräneattacken zum Behandlungsende sowie zum Ende der Beobachtungsphase. Auch die Migränetage waren in beiden Gruppen zum Zeitpunkt der Nachbeobachtung signifikant vermindert. Unter LD kam es zusätzlich zu einer signifikanten Reduktion der eingenommenen Akutmedikamente und der Schmerzintensität.

\subsubsection{Wirkung der manuellen Lymphdrainage auf Kopfschmerztage, Migräneattacken, Migränetage, akute Schmerzmedikation und Schmerzintensität}

Unter manueller Lymphdrainage nahmen die Kopfschmerztage zum Ende die Behandlung sowie vier Wochen nach Ende der Behandlung ab. Die Unterschiede waren jedoch im Vergleich mit der Kontrollgruppe nicht signifikant. Hier können neben der Migräne zusätzliche Kopfschmerzen vom Spannungstyp bestanden haben, bei denen möglicherweise die Lymphdrainage keine Wirkung hat.

Die manuelle Lymphdrainage führte am Beobachtungsende bei $38 \%$ der Patienten zu einer mindestens 50\%igen Reduktion der Migränetage. Die Responderrate bzgl. der Migränetage war im Vergleich mit der Kontrollgruppe zum sekundären Endpunkt, also direkt nach dem Behandlungsende, signifikant, ebenso die Differenz der Migränetage. Am Ende der Nachbeobachtung zeigte sich kein signifikanter Unterschied mehr in der Responderrate, jedoch in der Differenz der Migränetage. Das könnte bedeuten, dass die Lymphdrainage zwar signifikant die Migränetage am Ende der Behandlung reduziert, die Wirkung aber nach 4 Wochen langsam wieder nachlässt.

Die Responderrate bzgl. der Migräneattacken war nicht signifikant unterschiedlich zwischen den drei Gruppen, die Differenz der Migräneattacken zeigte jedoch sowohl zum Behandlungsende als auch zum Zeitpunkt der Nachbeobachtung eine signifikante Reduktion unter manueller Lymphdrainage. Das deutet darauf hin, dass die Lymphdrainage signifikant zur akuten Reduktion von Migräneattacken sowie 
als Prophylaxe wirksam ist. Hier sollten jedoch zur besseren Beurteilung der Prophylaxe weitere Untersuchungen mit längerer Beobachtungsphase (z.B. 12 bis 24 Wochen) durchgeführt werden.

Eine signifikante Reduktion der Schmerzmedikation, die bei Migräneattacken akut eingenommen wurden, konnte unter manueller Lymphdrainage sowohl zum Behandlungsende als auch zum Ende der Beobachtungsphase nachgewiesen werden. Hier könnte ein Zusammenhang mit der Abnahme der Migräneattacken und der Migränetage bestehen. Zusätzlich muss erwähnt werden, dass die Intensität der Schmerzen in der LD-Gruppe im Verlauf signifikant abnahm, was neben der Reduktion der Migräneattacken und -tage eine Erklärung für die Abnahme der Zahl der eingenommenen Schmerzmedikamente sein kann.

\subsubsection{Wirkung der klassischen Massage auf Kopfschmerztage, Migränetage, Migräneattacken, akute Schmerzmedikation und Schmerzintensität}

Unter klassischer Massage nahmen die Kopfschmerztage ebenfalls am Ende die Behandlung sowie am Ende der Beobachtungsphase ab. Die Unterschiede waren zwischen den Gruppen jedoch nicht signifikant. Hier können wie in der LD-Gruppe neben der Migräne zusätzliche Kopfschmerzen vom Spannungstyp bestanden haben, bei denen möglicherweise die klassische Massage keine Wirkung hat.

Die klassische Massage führte am Beobachtungsende ebenfalls bei $38 \%$ der Patienten zu einer mindestens 50\%igen Reduktion der Migränetage. Die Responderrate bzgl. der Migränetage war im Vergleich mit der Kontrollgruppe zu beiden Endpunkten nicht signifikant. Signifikant war jedoch die Differenz der Migränetage zum Beobachtungsende, nicht jedoch direkt nach der Behandlung. Das könnte bedeuten, dass sich die Wirksamkeit der klassischen Massage auf die Reduktion der Migränetage erst mit einer gewissen Verzögerung einstellt.

Migräneattacken konnten unter klassischer Massage signifikant sowohl zum Ende der Behandlung als auch am Ende der Beobachtungsphase reduziert werden während die Responderrate bzgl. Migräneattacken nicht unterschiedlich war. Also auch die klassische Massage ist während der Behandlung sowie vier Wochen nach der Behandlung wirksam in der Migräneprophylaxe. In einer randomisierten Studie von Lawler und Cameron (2004) konnte ebenfalls nachgewiesen werden, dass die klassische Massage über einen Interventionszeitraum von 6 Wochen (45 min Behandlung 1x/Woche) und drei Wochen danach (Nachbeobachtungsphase von 3 Wochen) zu einer signifikanten Reduktion der Migräneattacken im Vergleich zu einer Kontrollgruppe geführt hat. 
Trotz Abnahme der Migräneattacken unter klassische Massage war hier weder zum Behandlungsende noch am Ende der Beobachtungsphase eine signifikante Reduktion der eingenommen Schmerzmedikation im Vergleich zur Kontrollgruppe nachweisbar. Die Schmerzintensität war zwar geringer in beiden Phasen im Vergleich zur Kontrollgruppe, jedoch nicht signifikant.

Dies ist mit den Ergebnissen von Lawler und Cameron (2004) vergleichbar, wo ebenfalls kein signifikanter Unterschied zwischen klassischer Massage und einer Kontrollgruppe bezüglich der Abnahme der Schmerzmedikation und der Schmerzintensität gefunden werden konnte.

\subsubsection{Wirkung der manuellen Lymphdrainage auf Begleitsymptome}

Eine signifikante Reduktion von Photophobie im Verlauf weist auf den prophylaktischen Effekt der Lymphdrainage hin. Dieser Effekt war am Ende der Behandlung zwar geringer als am Ende der Nachbeobachtungsphase, jedoch aber effektiver als in Kontrollgruppe.

Hierzu liegen keine Studien zum Vergleich vor.

\subsubsection{Wirkung der klassischen Massage auf Begleitsymptome}

Die klassische Massage führte ebenfalls signifikant zu einer Reduktion der Photophobie im Verlauf während es in der Wartegruppe zu keiner signifikanten Abnahme von Begleitsymptomen kam. Auch die klassische Massage lässt somit einen prophylaktischen Effekt auf die Bedleitsymptome der Migräne vermuten.

Dieser Effekt auf die Begleitsymptome passt zu der Abnahme der Migräneattacken und Migränetage als Haupteffekt der klassischen Massage und Lymphdrainage. Weitere Studien mit längerer Nachbeobachtungsphase sind zu Untersuchung die Wirksamkeit der Lymphdrainage bei Migräne und ihren Begleitsymptomen erforderlich.

\subsubsection{Wirkung der manuellen Lymphdrainage und klassischen Massage auf psychometrische Parameter}

In allen drei Gruppen kam es zu einer signifikanten Abnahme der Beeinträchtigung durch Kopfschmerzen, gemessen mit dem IBK. Bei der Beeinträchtigung durch Kopfschmerzen hatten die Patienten unter Lymphdrainage eine Verbesserung des gesamten IBK-Scores um 14,7 Punkte zum Ende der Behandlung und um 14,3 Punkte zum Ende der Nachbeobachtung. Dies ist zu einer relevanten signifikanten Verbesserung nicht ausreichend. Nach „The Henry Ford Hospital Headache 
Disability Inventory“ muss ein Gewinn von mindestens 29 Punkten vorliegen, um von einer relevanten Verbesserung ausgehen zu können (Jacobson et al. 1994). Außerdem war auch eine Verbesserung in der Kontrollgruppe von im Mittel 11,4 Punkten zum Behandlungsende und 12,7 Punkten zum Ende der Nachbeobachtung zu beobachten. Unter der klassischen Massage war die Verbesserung noch geringer als in der Wartegruppe (9,2 bzw. 10,3 Punkte). Das bedeutet, die Lymphdrainage und die klassische Massage haben keine bessere Wirkung auf die Beeinträchtigung durch Kopfschmerzen als die Kontrollgruppe, und in beiden Gruppen war keine relevante Verbesserung zu erreichen. Das bedeutet, die akute Beeinträchtigung durch die Kopfschmerzen kann weder durch Lymphdrainage noch durch klassische Massage beeinflusst werden.

Die Lymphdrainage verbessert die Leistungsfähigkeit bzw. reduziert die verlorenen Tage wegen Migräne gemessen durch Midas signifikant im Vergleich zur KM und zur Kontrollgruppe. Der Effekt ist vier Wochen nach Ende der Behandlung (Nachbeobachtungsphase) ausgeprägter als am Ende der Behandlung. Ein wesentlicher Unterschied in den verlorenen Tagen gemessen durch Midas zwischen klassischer Massage und Kontrollgruppe war nicht zu beobachten. Das bedeutet, die Lymphdrainage hat eine positive Wirkung bezüglich der verlorenen Tage wegen Migräne für mindestens vier Wochen nach Ende der Therapie. Der Effekt ist stark von einem einzigen Ausreißer (mit 164 Tagen zur Baseline) geprägt, so dass dieses Ergebnis mit Vorsicht interpretiert werden sollte. Hier sind noch weitere Studien mit längerer Nachbeobachtungsphase und größerer Fallzahl erforderlich, um die prophylaktische Wirkung der Lymphdrainage auf die Leistungsfähigkeit bzw. verlorene Tage durch Kopfschmerzen zu beweisen.

Es ist bekannt, dass eine Komorbidität zwischen Migräne und bipolarer Depression besteht (LantériMint et al. 2005). Als Messparameter für bestehende depressive Symptome ist in dieser Studie die Center for Epidemiological Studies Depression Scale (CES-D) verwendet worden. Die Patienten in allen Gruppen hatten weder in der Interventionsphase noch in der Nachbehandlung im Mittel auffällige Werte. Im Vergleich mit der Kontrollgruppe, die auch am Ende der Nachbeobachtungsphase noch höhere CES-D-Werte von im Mittel 16,7 hatten, waren die Symptome im Verlauf der Behandlung mit Lymphdrainage sowie klassischer Massage abnehmend (Differenz um 36\% und 26\%), in der KMGruppe signifikant. Diese Wirkung ließ zwar vier Wochen nach Ende der Behandlung (Nachbeobachtung T4) etwas nach (Differenz um 25\% und 21\%), der CES-D-Wert blieb aber im Vergleich mit der Kontrollgruppe in den Behandlungsgruppen signifikant niedriger. Das bedeutet, beide Therapiemaßnahmen zeigen einen positiven Einfluss bzgl. der Reduktion depressiver Symptome, insbesondere zum Behandlungsende. Dieses ist besonders bemerkenswert, da in der CES-D keine 
Frage zum Schmerzempfinden enthalten ist und hierdurch somit keine Beeinflussung entstanden sein kann.

Die Wirkung der Lymphdrainage und der klassischen Massage auf die Lebensqualität der Migränepatienten wurde in dieser Studie durch den SF-36 mit der körperlichen und psychischen Summenskala sowie acht Subskalen bewertet. In der LD-Gruppe und in der KM-Gruppe zeigte sich eine signifikante Verbesserung in der körperlichen Summenskala im Verlauf, hier besonders in der körperlichen Rollenfunktion und bei körperlichen Schmerzen. In der KM-Gruppe zeigte sich ebenfalls eine signifikante Verbesserung der psychischen Summenskala im Verlauf, diese war in der LD-Gruppe knapp nicht signifikant, hier vor allem in der sozialen Funktionsfähigkeit. Interessanterweise kam es auch in der Wartegruppe zu einer signifikanten Verbesserung in der körperlichen Summenskala, ebenfalls in der körperlichen Rollenfunktion und bei körperlichen Schmerzen, während sich hier keine Veränderungen der psychischen Werte zeigten. Die Werte zwischen den Gruppen waren in der körperlichen Summenskala zu keinem Untersuchungszeitpunkt unterschiedlich, in der körperlichen Rollenfunktion und in der sozialen Funktionsfähigkeit waren die Werte in den Behandlungsgruppen jedoch besser als in der Kontrollgruppe. Dieses deutet darauf hin, dass Lymphdrainage und klassische Massage Verbesserungen der Lebensqualität, insbesondere der körperlichen Rollenfunktion und der körperlichen Schmerzen bewirken, die über einer Verbesserung durch reine Zuwendung liegen. Hierzu müssten jedoch längere Verlaufsuntersuchungen an einer größeren Patientengruppe durchgeführt werden, insbesondere auch deshalb, da sich die Lebensqualität erst in längeren Zeiträumen relevant verändert.

Abweichungen zu einer Normstichprobe, die im SF-36 Handbuch von der Autoren in bestimmten Bevölkerungsgruppen ermittelt wurde (hier: Normstichprobe Männer und Frauen im Alter zwischen 4150 Jahre), waren in beiden Summenskalen sowie in allen Subskalen zu erkennen (s. Abbildungen 40 und 41). Im Vergleich mit einer gesunden Kontrollgruppe weisen die untersuchten Migränepatienten also erwartungsgemäß eine geringere Lebensqualität in allen Bereichen auf, insbesondere in der Wahrnehmung der körperlichen Rollenfunktion und körperlicher Schmerzen. 

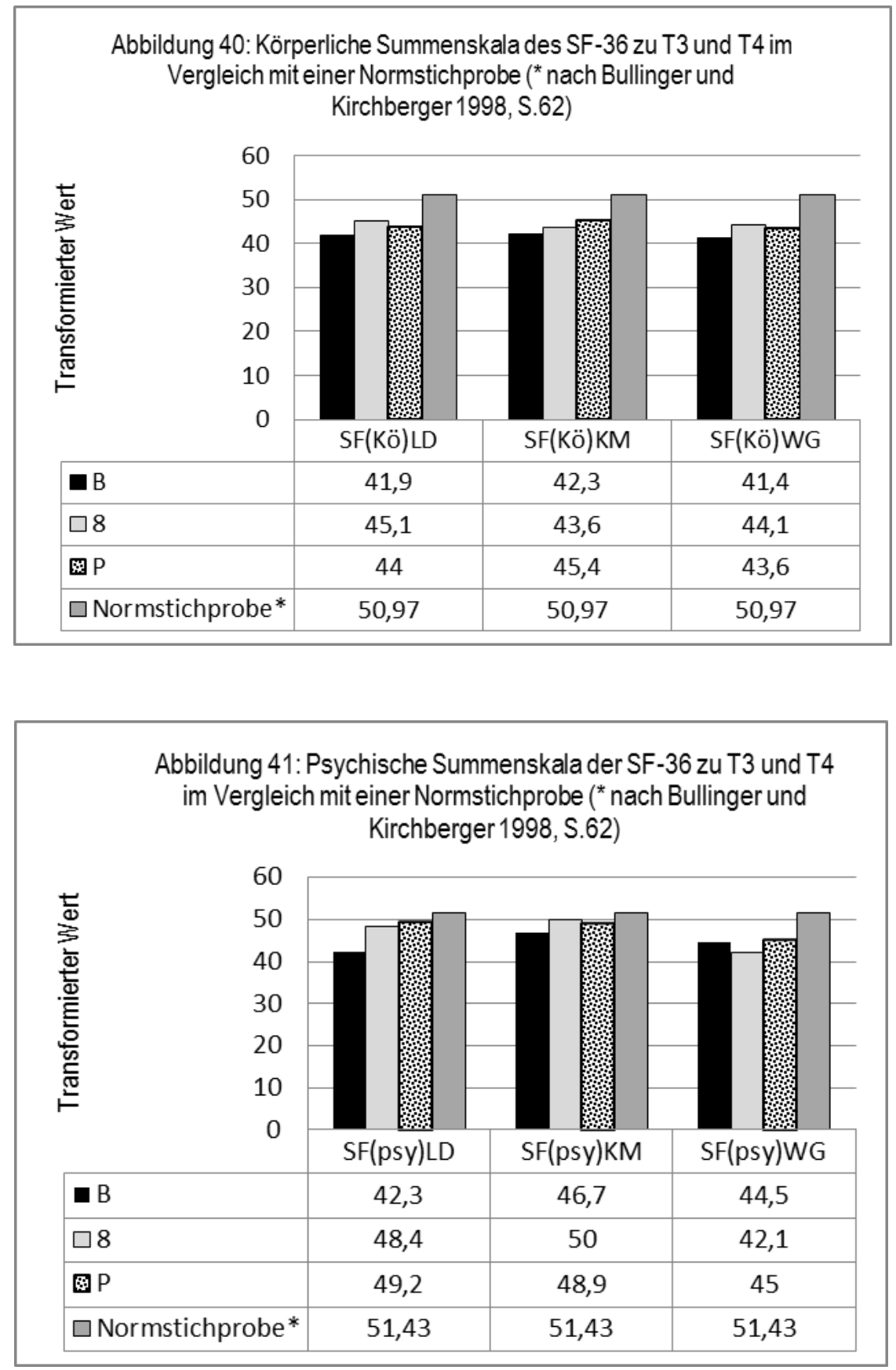
Diese Studie zeigte ebenfalls einen positiven Effekt der Lymphdrainage und klassischen Massage auf die Schlafqualität, gemessen durch den PSQI-Gesamtscore. Im Verlauf kam es in der KM-Gruppe zu einer signifikanten Reduktion des PSQI im Verlauf, dieser war in der LD-Gruppe knapp nicht signifikant. In der Wartegruppe kam es zu keiner Veränderung der Schlafqualität im Verlauf. Der positive Effekt der klassischen Massage wurde auch in der Arbeit von Lawler und Cameron (2004) während der Interventionsphase und drei Wochen später nachgewiesen. In diese Studie war die Verbesserung der Schlafqualität unter beiden Therapien (LD, KM) nur während der Interventionsphase zu beobachten. In der Beobachtungsphase vier Wochen nach Ende der Therapie war keine weitere Verbesserung im Vergleich zur Kontrollgruppe zu beobachten. Der Unterschied zwischen den Ergebnisse beider Studien könnte an den Messparametern liegen, da hier der gesamte PSQI-Score (der aus sieben Subskalen besteht) verwendet wurde, in der Studie von Lawler und Cameron (2004) wurde dahingegen eine Schlafqualitätsskala von 1 (sehr schlecht geschlafen) bis 5 (sehr gut geschlafen) benutzt. Problem des PSQI ist, ähnlich wie beim SF-36, dass er sich auf einen Zeitraum von vier Wochen vor der Befragung bezieht und kurzfristigere Veränderungen somit nicht erfasst werden können.

Bei der Beurteilung des Schweregrades der Migräne fand sich, gemessen durch den CGI, unter beiden Therapiemaßnahmen im Vergleich zur Wartegruppe eine signifikante Verbesserung im Verlauf, sowohl am Behandlungsende als auch am Ende der Nachbeobachtungsphase. Dieses zeigt eine in der Fremdbeurteilung signifikante Verbesserung der Migräne unter Lymphdrainage, aber auch unter klassischer Massage

Hauptproblem dieser ersten randomisierten, kontrollierten Parallelgruppenstudie zur Untersuchung der Wirksamkeit der manuellen Lymphdrainage im Vergleich zur klassischen Massage zur Prophylaxe der Migräne mit und ohne Aura sind die kleine Fallzahl und die kurze Beobachtungsphase. In einer weiteren Studie mit einer größeren Fallzahl und einer längeren Beobachtungsphase sollten die hier gefundenen Ergebnisse verifiziert werden. Beide Verfahren können nach bisherigem Wissen als wirkungsvolle nichtmedikamentöse Therapiemaßnahmen zur Prophylaxe der Migräne mit und ohne Aura empfohlen werden mit einem möglicherweise leichten Vorteil der Lymphdrainage. 


\subsection{Ausblick}

Bei der vorliegenden Untersuchung handelt es sich um die erste kontrollierte, randomisierte Studie zu Untersuchung der Wirksamkeit der manuellen Lymphdrainage zur Prophylaxe der Migräne mit und ohne Aura. Es konnte gezeigt werden, dass die manuelle Lymphdrainage eine positive Wirkung auf Migräneattacken, Migränetage, Schmerzmedikation, Schmerzintensität, Begleitsymptome, Leistungsfähigkeit, Schlafqualität und Lebensqualität hat. Diese Wirkung war sowohl während der Interventionsphase als auch in der Nachbeobachtungsphase zu beobachten. Es gab Unterschiede, die in weiteren Studien mit längerer Nachbeobachtungszeit und größerer Fallzahl untersucht werden sollten, um den prophylaktischen Effekt der Lymphdrainage auch über einen längeren Zeitraum sicher zu stellen. Außerdem sind weitere Studien erforderlich, um die Ätiologie und den Mechanismus dieser Wirksamkeit der Lymphdrainage auf der Basis der Pathophysiologie der Migräne-Kopfschmerzen zu untersuchen. Unterschiede zwischen Lymphdrainage und klassischer Massage bezüglich der Wirksamkeit bei Migräne konnten in dieser Studie an mehreren Stellen gezeigt werden. Hierbei scheint die manuelle Lymphdrainage etwas wirksamer als die klassische Massage zu sein. Dieses muss ebenfalls in weiteren Studien mit Wartegruppen neben Shamgruppen zur Untersuchung des PlaceboEffektes untersucht werden. 


\section{Zusammenfassung der Hauptparameter}

Fragestellung: Methoden der physikalischen Therapie zur Prophylaxe der Migräne sind, obwohl häufig angewendet, bislang nur unzureichend untersucht. Ziel dieser Studie ist die Untersuchung, ob eine Behandlung mit Lymphdrainage (LD) oder klassischer Rückenmassage (KM) als Prophylaxe gegen Migräne mit und ohne Aura wirksam ist.

Material und Methoden: Randomisierte, kontrollierte Parallelgruppenstudie von 64 Patienten (57 Frauen, mittleres Alter 45 +/- 10 Jahre) in drei Gruppen: Die erste Gruppe erhielt LD ( $n=21)$, die zweite KM ( $n=21)$ und die dritte keine Therapie (Wartegruppe, WG, $n=22$ ). Es erfolgte eine 30-minütige Therapie $1 \mathrm{x}$ pro Woche über 8 Wochen. Vor Beginn und nach Ende der 8-wöchigen Therapie erfolgte jeweils eine 4-wöchige Beobachtungsphase, die für die Ermittlung der Wirksamkeit verglichen wurden. Bei Aufnahme in die Studie und alle vier Wochen wurden alle Patienten von einem Arzt untersucht. Als Messparameter diente der kontinuierlich geführte Kopfschmerzkalender der DMKG. Primäre Endpunkte waren die Responderrate (mind. 50\%ige Reduktion der Migräneattacken und Kopfschmerztage) und die Differenz der Migränetage, der Kopfschmerztage und in der Einnahme von Akutmedikamenten zwischen den beiden Beobachtungsphasen. In einer sekundären Endpunktanalyse wurden dieselben Parameter zwischen der Baseline und dem Behandlungsende verglichen.

Ergebnisse: Zum Beobachtungsende ergaben sich: 1) keine signifikanten Unterschiede der Responderraten; 2) ein signifikanter Unterschied in der Differenz der Migräneattacken $(p=0,016)$ und der Migränetage $(p=0,019)$ zwischen LD und WG $(p=0,006$ bzW. $p=0,015)$ sowie zwischen KM und WG $(p=0,042$ bzw. $p=0,016)$. Im Verlauf zeigte sich eine signifikante Abnahme der Zahl der eingenommenen Akutmedikamente in der LD-Gruppe im Vergleich zu den beiden anderen Gruppen ( $p=0,004)$.

Zum Behandlungsende ergaben sich: 1) keine signifikanten Unterschiede der Responderraten; 2) ein signifikanter Unterschied der Differenz der Migräneattacken zwischen LD und WG $(p=0,013)$, und zwischen KM und WG ( $p=0,033) ; 3)$ kein Unterschied der Differenz der Migränetage $(p=0,162)$ und der Kopfschmerztage $(p=0,290)$.

Diskussion: In dieser Studie führten beide physikalischen Therapiemaßnahmen KM und LD zu einer Reduktion der Migräneattacken zum Behandlungsende sowie zum Ende der Beobachtungsphase. Nur unter LD kam es zusätzlich zu einer Reduktion der eingenommenen Akutmedikamente. 
Schlussfolgerung: LD und KM sind im Vergleich zu einer Wartegruppe wirksam zur Prophylaxe der Migräne mit und ohne Aura. Im direkten Vergleich zeigen sich in einigen Parametern signifikante Vorteile der LD gegenüber der KM. Weitere Studien mit größerer Patientenzahl müssen diese Pilotergebnisse untermauern. 


\section{Anhang}

\subsection{Anhang 1: Patienteninformation}

\section{Randomisierte, kontrollierte Parallelgruppenstudie zur Untersuchung der Wirksamkeit der Lymphdrainage zur Prophylaxe der Migräne mit und ohne Aura}

Sehr geehrte Teilnehmerin, sehr geehrter Teilnehmer!

Wir möchten Sie bitten, an oben genannter wissenschaftlicher Untersuchung teilzunehmen und möchten Ihnen den Ablauf der Studie anhand dieses Informationsbogens erläutern.

Die Migräne stellt eine der häufigsten Kopfschmerzerkrankungen dar, etwa 10\% aller Menschen leiden darunter. Für die Akutbehandlung und Prophylaxe (Vorbeugung) der Migräne stehen heute zahlreiche wirksame Medikamente zur Verfügung. Methoden der physikalischen Therapie zur Prophylaxe der Migräne sind, obwohl häufig angewendet und oft als wirksam beschrieben, bislang nur unzureichend untersucht. Insbesondere für die Lymphdrainage und die klassische Massage gibt es Hinweise, dass sie in der Prophylaxe der Migräne wirksam sein können.

Bei der Lymphdrainage handelt es sich um eine periphere entstauende manuelle Therapie durch weiche, gewebeschonende Massagegriffe überwiegend an der Körperoberfläche. Die Behandlung orientiert sich entlang des anatomischen Verlaufes von Lymphgefäßen in Abflussrichtung. Der Daumen und die Langfinger des Therapeuten führen auf dem Gewebe großflächige, gleich- oder gegensinnig kreisende Bewegungen mit abwechselnd stufenlos ansteigendem und dann wieder abfallendem Druck aus, dieses wird in der Regel als angenehm empfunden. Diese anerkannte manuelle Methode soll hinsichtlich ihrer Wirksamkeit zur Prophylaxe der Migräne untersucht werden. Im Vergleich dazu soll untersucht werden, ob die klassische Massage wirksam ist zur Prophylaxe der Migräne. Massagen gehören zu den ältesten Behandlungsverfahren überhaupt und sind in allen Kulturen verwurzelt. Dabei handelt es sich um eine mechanische Manipulation bzw. Stimulation der Weichteilgewebe (Haut, Unterhautbindegewebe, Muskulatur, Bänder, Sehnen, Knochenhaut), d.h. die Hand ertastet und behandelt einen Befund. In relativ monotoner, sich wiederholender Weise werden rhythmische DruckZugbeanspruchungen, Streichungen, Reibungen und Dehnungen auf die Körperoberfläche mit dem Ziel, Muskeln, Kreislauf und Nervensystem zu beeinflussen bzw. zu stimulieren, angewendet.

Hierbei soll untersucht werden, ob die Anzahl der Migräneattacken unter der Behandlung mit Lymphdrainage oder klassischer Massage abnimmt und ob die Schmerzintensität und die Anzahl der eingenommenen Akutmedikamente reduziert werden können. 
Um dieses zu untersuchen, werden Sie gebeten, an dieser Studie teilzunehmen. Sie selbst werden möglicherweise nicht von dieser Studie profitieren, die Ergebnisse ermöglichen es uns jedoch, weiterführende Erkenntnisse über alternative Therapiemöglichkeiten der Migräne zu gewinnen. Sie werden gebeten, zunächst über vier Wochen ein Kopfschmerztagebuch zu führen und einige Fragebögen bzgl. der Schmerzen und der Lebensqualität auszufüllen. Dann erfolgt entweder eine 8wöchige Phase der Therapie mit Lymphdrainage oder klassischer Massage einmal in der Woche à 30 Minuten oder eine 8-wöchige Wartezeit. Im Anschluss daran werden Sie nochmals gebeten, über vier weitere Wochen ein Kopfschmerztagebuch zu führen und sich abschließend nochmals zur Nachbeobachtung und Bewertung des Therapieerfolges bei uns vorzustellen. Die Gruppe, die zu Beginn noch keine Therapie erhalten hat, beginnt nach der 8-wöchigen Wartezeit mit der 8-wöchigen Therapie und wird dann nochmals nach vier Wochen untersucht, wie die erste Gruppe, die von Anfang an eine Therapie erhalten hat. Zum Zeitpunkt der Aufnahme in die Studie und alle vier Wochen werden Sie von einer Ärztin/einem Arzt gesehen und kurz untersucht und werden gebeten, die Fragebögen auszufüllen. Die Studiendauer wird für Sie also maximal sieben Monate betragen, in denen Sie auf jeden Fall über einen Zeitraum von acht Wochen eine wöchentliche Lymphdrainage oder klassische Massage à 30 Minuten erhalten werden.

Selbstverständlich ist es Ihnen jederzeit möglich, ohne Angabe von Gründen und ohne dass Ihnen hieraus Nachteile entstehen, von der Teilnahme an der Studie zurückzutreten. Nachhaltige Nebenwirkungen auf Ihre Erkrankung sind durch diese Untersuchung nicht zu erwarten.

Falls einer der folgenden Punkte auf Sie zutrifft, ist eine Teilnahme an der Studie nicht möglich:

1. Alter $\leq 18$ Jahre;

2. Schwangerschaft und Stillzeit;

3. weniger als 2 Migräne-Attacken pro Monat;

4. anderer idiopathischer oder sekundärer Kopfschmerz;

5. relevante neurologische, psychiatrische oder internistische Erkrankung;

6. Änderung der medikamentösen Medikation zur Migräneprophylaxe;

7. Einnahme von Analgetika an mehr als 3 Tagen pro 4 Wochen wegen anderer chronischer Schmerzen;

8. aktuelle Kortisontherapie;

9. Medikamentenmissbrauch;

10. Teilnahme an einer anderen Studie in den vergangenen 3 Monaten;

11. Kontraindikation der manuellen Lymphdrainage oder klassischen Massage (z. B. schwere dekompensierte Herz-/Kreislauferkrankungen (NYHA III \& IV), lokale Entzündungen, Verletzungen oder Raumforderungen im Behandlungsgebiet, Bradykardie mit HF < 50/min., ausgeprägte Arrhythmie, Carotis-Sinus-Syndrom, Hyperthyreose);

12. Vorliegen einer gesetzlichen Betreuung.

Die aus der Studie gewonnenen Daten werden anonymisiert wissenschaftlich ausgewertet. Alle Erfordernisse des Datenschutzes werden dabei beachtet. Die möglichen Risiken sind als sehr gering einzustufen. 


\subsection{Anhang 2: Einverständniserklärung}

\section{Randomisierte, kontrollierte Parallelgruppenstudie zur Untersuchung der Wirksamkeit der Lymphdrainage zur Prophylaxe der Migräne mit und ohne Aura}

Frau/Herr Dr. hat mir heute anhand der Hinweise auf dem Informationsbogen für Teilnehmerinnen und Teilnehmer an der 0 . g. Studie die Durchführung der Untersuchung erläutert.

Ich habe diesbezüglich keine weiteren Fragen mehr und willige hiermit in die dargestellten Untersuchungen ein. Alle mich interessierenden Fragen wurden ausreichend beantwortet.

Ich erkläre darüber hinaus, dass ich alle Angaben zur Krankengeschichte wahrheitsgemäß gemacht habe.

Mir ist bekannt, dass ich jederzeit ohne Angabe von Gründen die weitere Untersuchung ablehnen bzw. meine Einverständniserklärung widerrufen kann, ohne dass mir daraus Nachteile entstehen.

Ich bin bereit, an der Studie teilzunehmen.

Ich bin damit einverstanden, dass Daten, welche sich aus der Studie ergeben, ohne Erwähnung meiner Identität anonym veröffentlicht oder an zuständige Behörden weitergeleitet werden. Alle Befunde sind vertraulich und werden unter strikter Einhaltung des Datenschutzes gehandhabt. Eine Kopie dieser Erklärung wurde mir überreicht.

Bremen, den

Name der/des aufklärenden Ärztin/Arztes (in Druckbuchstaben) und Unterschrift

Name der/des Patientin/Patienten (in Druckbuchstaben) und Unterschrift 
7.3. Anhang 3: Standardisierter Erhebungsbogen der demographischen und klinischen Daten

Name:

Vorname:

Telefon:

Geb.:

Datum:

Teilnahme an Studie in vergangenen 3 Monaten?

$$
\begin{aligned}
& \text { ja } \square / \text { nein } \square \\
& \text { ja } \square \text { / nein } \square
\end{aligned}
$$

Teilnahme an dieser Studie?

Wenn nein, warum nicht?

Diagnose: Migräne mit $\square /$ ohne $\square$ Aura

Beginn:

Attackenfrequenz in letzten 3 Monaten:

Med. Prophylaxe?

Wenn ja, welche?

Seit wann?

Reduzierung der Attacken?

Vorherige Zahl der Attacken?

Besonderheiten:

Gewicht:

RR (sitzend):

Aktuelle Medikamente mit Dosis und Therapiebeginn:

Vorerkrankungen:
Lebensjahr

/ Monat

ja $\square /$ nein $\square$

ja $\square /$ nein $\square$

Größe:

Puls: 


\subsection{Anhang 4: Kopfschmerzkalender}
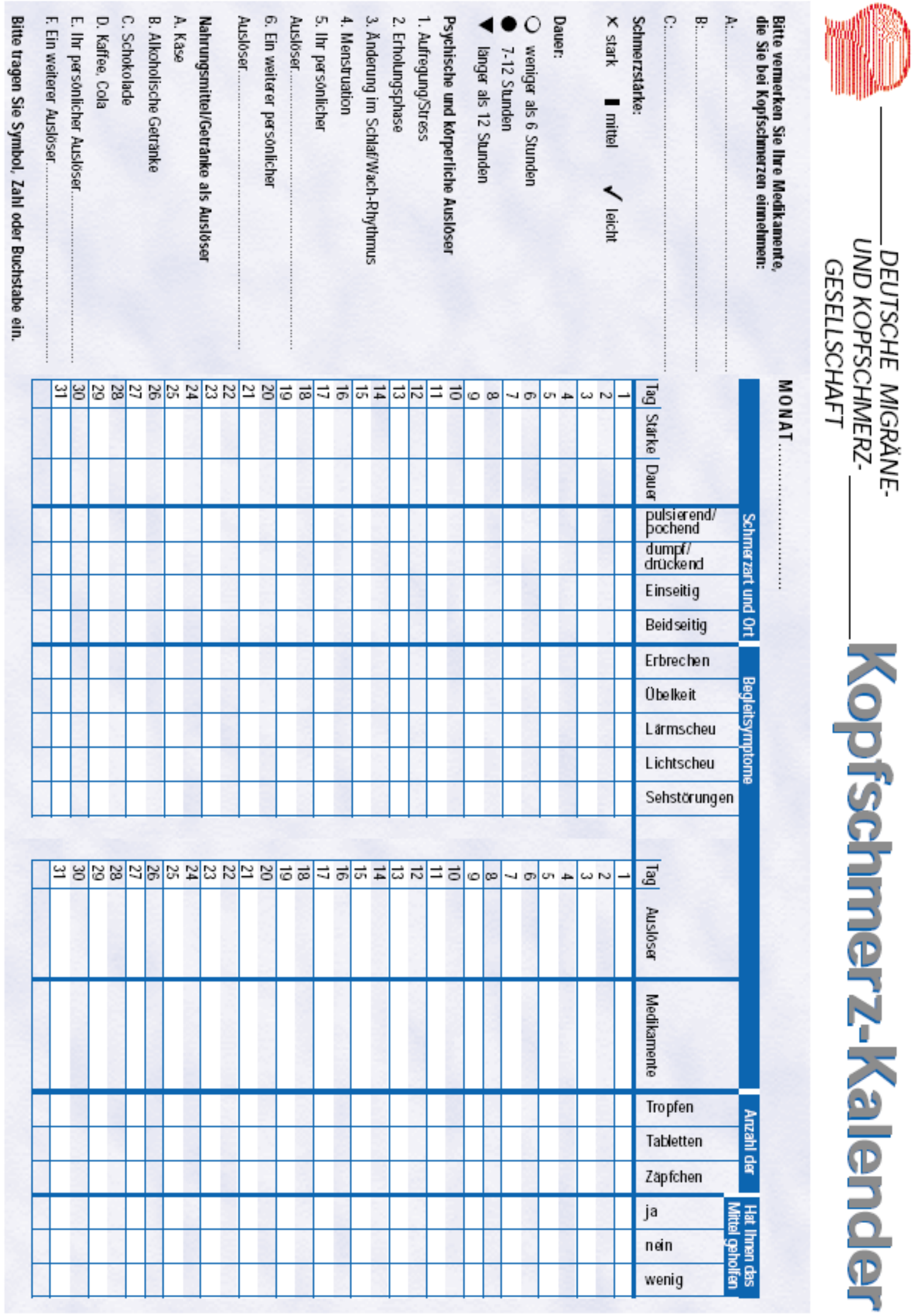


\subsection{Anhang 5: Inventar zur Beeinträchtigung durch Kopfschmerzen, IBK (Bauer et}

\section{al.1999)}

Mit folgenden Fragen sollen Sie Ihre Kopfschmerzen einschätzen.

Bitte beantworten Sie jede Frage mit „Ja“ "manchmal“ oder „nein“(Bitte kreuzen Sie ein Kästchen für jede Frage an)

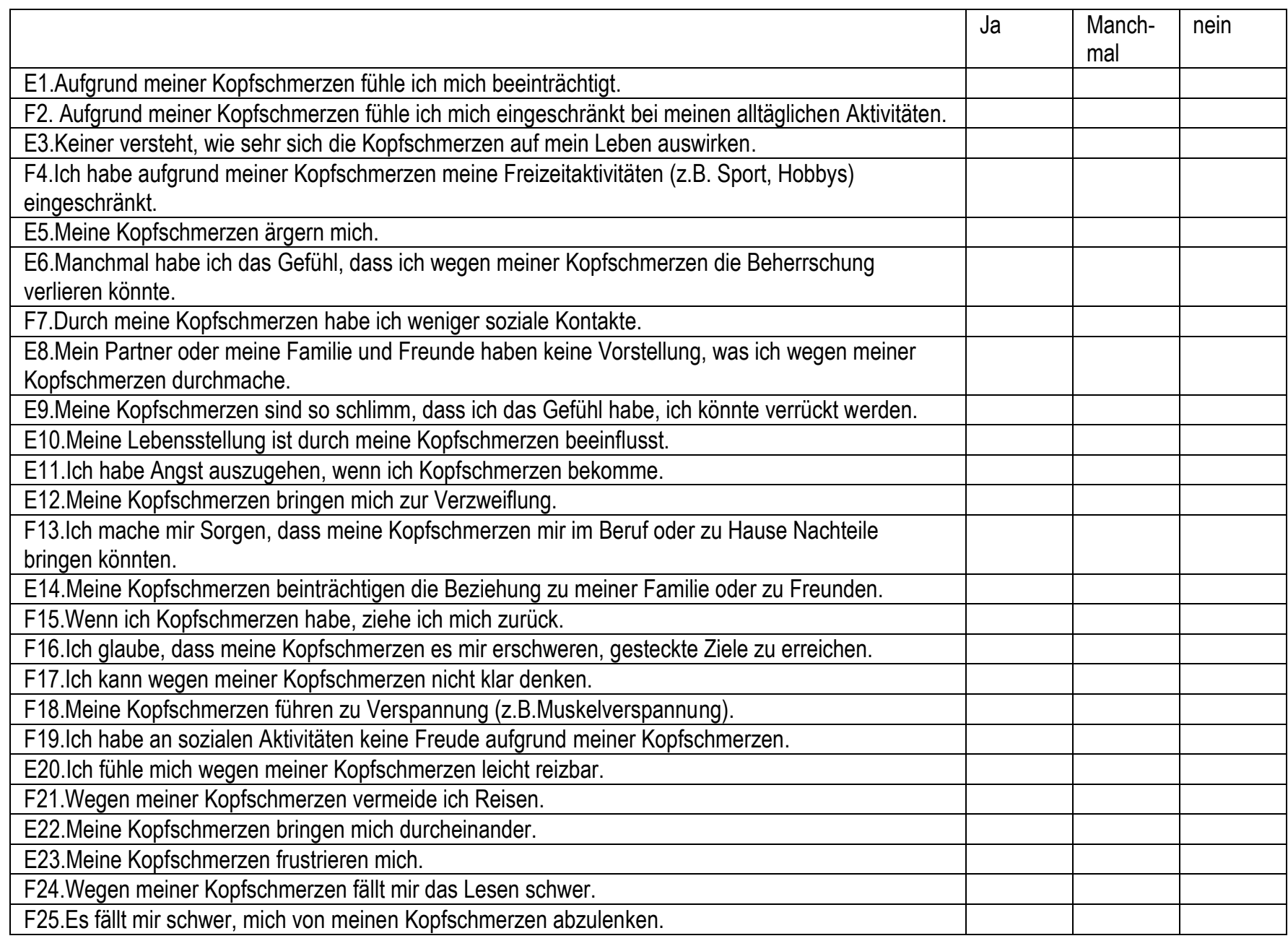

26. Wie stark sind Ihre Kopfschmerzen üblicherweise?

\begin{tabular}{|l|l|l|}
\hline leicht & mittel & schwer \\
\hline & & \\
\hline
\end{tabular}

27. An wieviel Tagen im Monat haben Sie Kopfschmerzen?

28. Seit wieviel Jahren leiden Sie an Kopfschmerzen? 


\subsection{Anhang 6: Migraine disability assessment, Midas Fragebogen (Stewart et al. 1999)}

Anleitung: Bitte beantworten Sie die folgenden Fragen über ALLE Kopfschmerzattacken, die Sie in den letzten drei Monaten hatten. Füllen Sie das Kästchen neben jeder Frage mit der entsprechenden Zahl aus. Schreiben Sie 0, wenn die Antwort negativ ist.

1. An wie vielen Tagen in den letzten drei Monaten sind Sie wegen

Kopfschmerzen nicht zur Arbeit gegangen?

2. An wie vielen Tagen war in den letzten drei Monaten Ihre Leistungsfähigkeit am Arbeitsplatz oder in der Schule um die Hälfte oder mehr eingeschränkt?

(Zählen Sie die Tage, die Sie bei Frage 1 angaben, NICHT dazu)

3. An wie vielen Tagen in den letzten drei Monaten konnten Sie wegen Ihrer Kopfschmerzen keine Hausarbeit verrichten?

4. An wie vielen Tagen in den letzten drei Monaten war Ihre Leistungsfähigkeit im Haushalt um die Hälfte oder mehr eingeschränkt?

(Zählen Sie die Tage, die Sie bei Frage 3 angaben, NICHT dazu)

5. An wie vielen Tagen in den letzten drei Monaten haben Sie an familiären, sozialen oder Freizeitaktivitäten wegen Ihrer Kopfschmerzen nicht teilnehmen können?

A An wie vielen Tagen hatten Sie in den letzten drei Monaten Kopfschmerzen? (Wenn die Kopfschmerzen länger als einen Tag angehalten haben, zählen Sie jeden Tag)

B Wie stark waren diese Kopfschmerzen?

Bitte geben Sie die Schmerzintensität auf einer Skala von 0 - 10 an.

( $0=$ keine Schmerzen, $10=$ unerträgliche Schmerzen $)$

Bitte zählen Sie die Tage der Fragen 1 - 5 zusammen, sobald Sie den Fragebogen vollständig ausgefüllt haben. (Die Fragen A und B bitte NICHT dazuzählen)

\begin{tabular}{lll}
\multicolumn{2}{l}{ Auswertung des MIDAS Fragebogens: } \\
Grad & Definition & Punkte \\
I & Wenig oder keine Beeinträchtigung & $0-5$ \\
II & Geringe Beeinträchtigung & $6-10$ \\
III & Mäßige Beeinträchtigung & $11-20$ \\
IV & Schwere Beeinträchtigung & $21+$ \\
\hline
\end{tabular}




\subsection{Anhang 7: Center for Epidemiologic Studies Depression Scale, CES-D (Andresen et al. 1994)}

Im nächsten Teil würde ich gerne wissen, wie Sie sich letzte Woche gefühlt haben. Bitte nennen Sie die Antwort, die Ihre Stimmung in der letzten Woche am besten beschreibt.

Während der letzten Woche...

...haben mich Dinge beunruhigt, die mir sonst nichts ausmachen

...hatte ich kaum Appetit

...konnte ich meine trübsinnige Laune nicht loswerden, obwohl mich meine Freunde/Familie versuchten aufzumuntern

...kam ich mir genauso gut vor, wie andere

...hatte ich Mühe mich zu konzentrieren

...war ich deprimiert/niedergeschlagen

...war alles anstrengend für mich

...dachte ich voller Hoffnung an die Zukunft

...dachte ich, mein Leben ist ein einziger Fehlschlag

...hatte ich oft Angst

...habe ich schlecht geschlafen

...war ich fröhlich gestimmt

...habe ich weniger als sonst geredet

...fühlte ich mich einsam

...waren die Leute unfreundlich zu mir

...habe ich das Leben genossen

...musste ich manchmal weinen

...war ich traurig

...hatte ich das Gefühl, dass mich die Leute nicht leiden können

...bin ich überhaupt nicht in die Gänge gekommen

\section{Antwortmöglichkeiten für alle Fragen:}

1 kaum oder überhaupt nicht (weniger als $1 \mathrm{Tag}$ )

2 manchmal (1-2 Tage)

3 öfter (3-4 Tage)

4 die meiste Zeit (5-7 Tage) 
7.8. Anhang 8: Lebensqualitätsfragebogen, SF-36 (Ware et al. 1993)

Fragebogen zum Gesundheitszustand

In diesem Fragebogen geht es um Ihre Beurteilung Ihres Gesundheitszustandes.

Der Bogen ermöglicht es nachzuvollziehen, wie sie sich fühlen und wie Sie im Alltag zurechtkommen. Bitte beantworten Sie jede der vorgelegten Fragen, indem Sie bei den Antwortmöglichkeiten die Zahl ankreuzen, die am besten auf Sie zutrifft.

1. Wie würden Sie Ihren Gesundheitszustand im Allgemeinen beschreiben?

(Bitte kreuzen Sie nur eine Zahl an!)

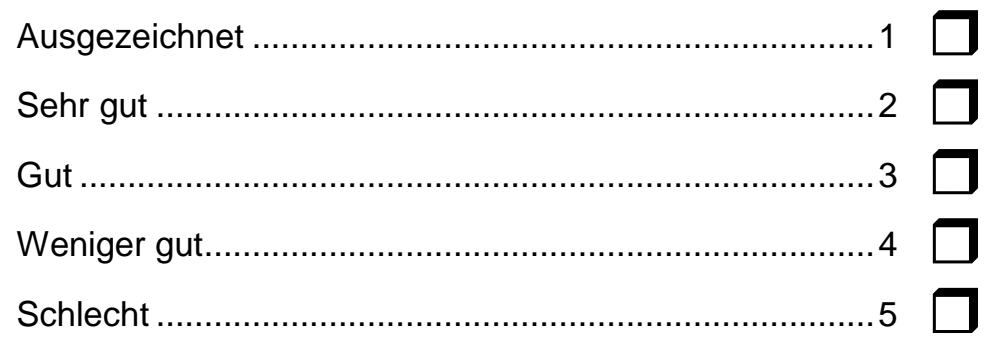

2. Im Vergleich zum vergangenen Jahr, wie würden Sie Ihren derzeitigen Gesundheitszustand beschreiben?

(Bitte kreuzen Sie nur eine Zahl a !)

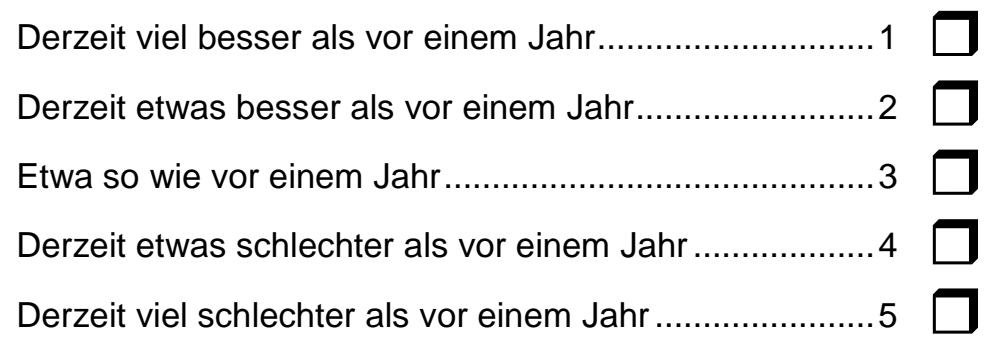

Im folgenden sind einige Tätigkeiten beschrieben, die Sie vielleicht an einem normalen Tag ausüben. Sind Sie durch Ihren derzeitigen Gesundheitszustand bei diesen Tätigkeiten eingeschränkt? Wenn ja, wie stark? (Bitte kreuzen Sie in jeder Zeile nur eine Zahl an!)

\section{Tätigkeiten}

3. anstrengende Tätigkeiten, z.B. schnell laufen, schwere Gegenstände heben, anstrengenden Sport treiben

4. mittelschwere Tätigkeiten, z.B. einen Tisch verschieben, staubsaugen, kegeln, Golf spielen

5. Einkaufstaschen heben + tragen

6. mehrere Treppenabsätze steigen

7. einen Treppenabsatz steigen

8. sich beugen, knien, bücken

9. mehr als $\mathbf{1} \mathbf{~ k m ~ z u ~ F u ß ~ g e h e n ~}$
Ja, stark eingeschränkt

1<smiles>C1CCC1</smiles>

1

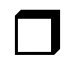

Ja, etwas eingeschränk

2

2

3

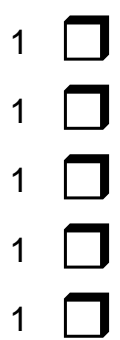

2

2

2

2

2
Nein,überhaupt nicht eingeschränkt

3 
10. mehrere Straßenkreuzungen weit zu Fuß gehen

11. eine Straßenkreuzung weit zu Fuß gehen

12. sich baden oder anziehen
1

1

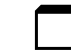

1
2

2

2
3

3

3

Hatten Sie in den vergangenen 4 Wochen aufgrund Ihrer körperlichen Gesundheit irgendwelche Schwierigkeiten bei der Arbeit oder anderen alltäglichen Tätigkeiten im Beruf bzw. zu Hause?

(Bitte kreuzen Sie in jeder Zeile nur eine Zahl an!)

\section{Schwierigkeiten}

13. Ich konnte nicht so lange wie üblich tätig sein

14. Ich habe weniger geschafft als ich wollte

15. Ich konnte nur bestimmte Dinge tun

16. Ich hatte Schwierigkeiten bei der Ausführung (z.B. ich mußte mich besonders anstrengen)
JA

Nein

Hatten Sie in den vergangenen 4 Wochen aufgrund seelischer Probleme irgendwelche Schwierigkeiten bei der Arbeit oder anderen alltäglichen Tätigkeiten im Beruf bzw. zu Hause (z.B. weil Sie sich niedergeschlagen oder ängstlich fühlten?

\section{Schwierigkeiten}

(Bitte kreuzen Sie in jeder Zeile nur eine Zahl an!)

17. Ich konnte nicht so lange wie üblich tätig sein

18. Ich habe weniger geschafft als ich wollte

19. Ich konnte nicht so sorgfältig wie üblich arbeiten

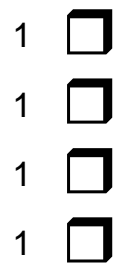

$2 \square$

$2 \square$

$2 \square$

$2 \square$

20. Wie sehr haben Ihre körperliche Gesundheit oder seelische Probleme in den vergangenen 4 Wochen Ihre normalen Kontakte zu Familienangehörigen, Freunden, Nachbarn oder zum Bekanntenkreis beeinträchtigt? (Bitte kreuzen Sie nur eine Zahl an!)

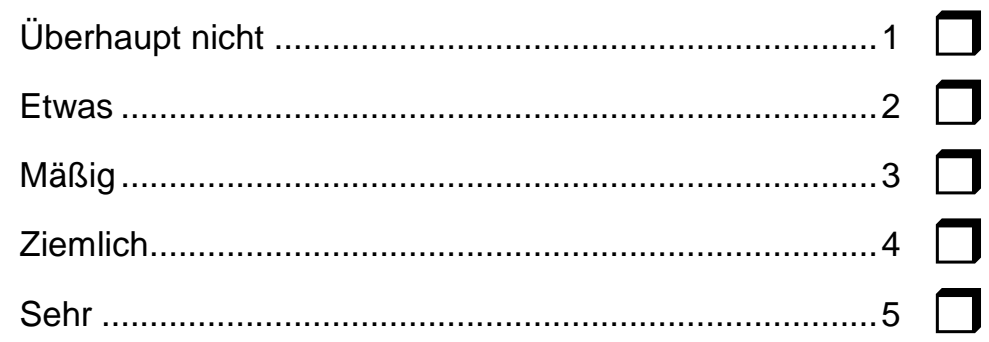


21. Wie stark waren Ihre Schmerzen in den vergangenen 4 Wochen?

(Bitte kreuzen Sie nur eine Zahl an!)

Ich hatte keine Schmerzen

1

2

3

4

5

Sehr stark

.6

22. Inwieweit haben die Schmerzen Sie in den vergangenen 4 Wochen bei der Ausübung Ihrer Alltagstätigkeiten zu Hause und im Beruf behindert ? (Bitte kreuzen Sie nur eine Zahl an!)

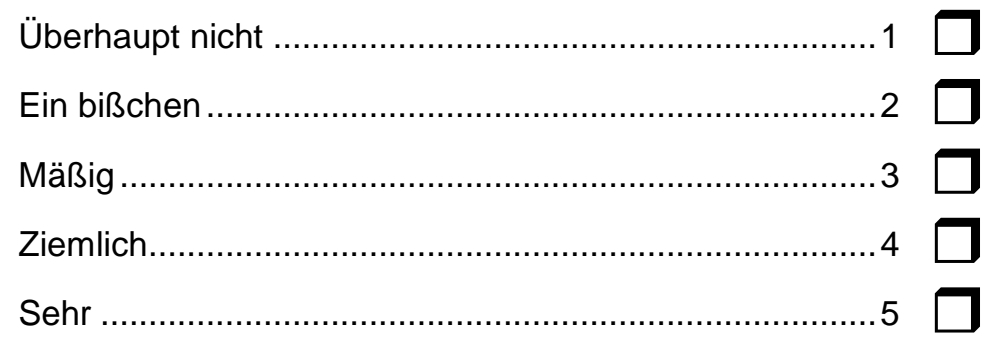

In diesen Fragen geht es darum, wie Sie sich fühlen und wie es Ihnen in den letzten 4 Wochen gegangen ist. (Bitte kreuzen Sie in jeder Zeile die Zahl an, die Ihrem Befinden am ehesten entspricht). Wie oft waren Sie in den vergangenen 4 Wochen

(Bitte kreuzen Sie in jeder Zeile nur eine Zahl an!)

\section{Befinden}

23. ...voller Schwung ?

24. ....sehr nervös ?

25. ...so niedergeschlagen, dass Sie nichts aufheitern konnte?

26. ...ruhig und gelassen?

27. ...voller Energie ?

28. ...entmutigt und traurig?

29. ...erschöpft?

30. ...glücklich ?

31. ...müde?

\section{Immer}

Meistens

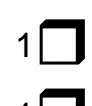

$2 \square$

oft

$1 \square$

$2 \square$

$3 \square$

$1 \square$

$2 \square$

$3 \square$

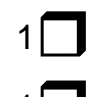

$2 \square$

$3 \square$

$2 \square$

$3 \square$

$1 \square$

$1 \square$

$2 \square$

$3 \square$

$2 \square$

$3 \square$

$1 \square$

$2 \square$

$1 \square$
Manch-

Selten

mal

$4 \square$

$4 \square$

$4 \square$

$5 \square$

$5 \square$

$5 \square$

$4 \square$

$5 \square$

$4 \square$

$5 \square$

$4 \square$

$5 \square$

$4 \square$

$5 \square$

$4 \square$

$5 \square$

$4 \square$ 
32. Wie häufig haben Ihre körperliche Gesundheit oder seelische Probleme in den vergangenen 4 Wochen Ihre Kontakte zu anderen Menschen (Besuche bei Freunden, Verwandten usw.) beeinträchtigt? (Bitte kreuzen Sie nur eine Zahl an !)

Immer

Inwieweit trifft jede der folgenden Aussagen auf Sie zu?

\begin{tabular}{|c|c|c|c|c|c|}
\hline Aussagen & $\begin{array}{l}\text { Trifft } \\
\text { ganz zu }\end{array}$ & $\begin{array}{l}\text { Trifft weit- } \\
\text { gehend zu }\end{array}$ & $\begin{array}{l}\text { Weiß } \\
\text { nicht }\end{array}$ & $\begin{array}{l}\text { Trifft weitgeh- } \\
\text { end nicht zu }\end{array}$ & $\begin{array}{l}\text { ur eine Zahl an! } \\
\text { Trifftüber } \\
\text { haupt nicht zu }\end{array}$ \\
\hline $\begin{array}{l}\text { 33. Ich scheine etwas leichter } \\
\text { als andere krank zu werden }\end{array}$ & & & & & \\
\hline $\begin{array}{l}\text { 34. Ich bin genauso gesund wie } \\
\text { alle anderen die ich kenne }\end{array}$ & & & & & \\
\hline $\begin{array}{l}\text { 35. Ich erwarte, daß meine } \\
\text { Gesundheit nachläßt }\end{array}$ & & & & & \\
\hline $\begin{array}{l}\text { 36. Ich erfreue mich ausge- } \\
\text { zeichneter Gesundheit }\end{array}$ & & & & & \\
\hline
\end{tabular}




\subsection{Anhang 9: Pittsburgh Sleep Quality Index, PSQI (Buysse et al. 1989)}

Die folgenden Fragen beziehen sich auf Ihre üblichen Schlafgewohnheiten und zwar nur während der letzten vier Wochen. Ihre Antworten sollten möglichst genau sein und sich auf die Mehrzahl der Tage und Nächte während der letzten vier Wochen beziehen. Beantworten Sie bitte alle Fragen.

1. Wann sind Sie während der letzten vier Wochen gewöhnlich abends zu Bett gegangen?

2. Wie lange hat es während der letzten vier Wochen gewöhnlich gedauert, bis Sie nachts eingeschlafen sind?

3. Wann sind Sie während der letzten vier Wochen gewöhnlich morgens aufgestanden?

4. Wieviele Stunden haben Sie während der letzten vier Wochen pro Nacht tatsächlich geschlafen?

(Das muß nicht mit der Anzahl der Stunden, die Sie im Bett verbracht haben, übereinstimmen.) übliche Uhrzeit:

in Minuten:

übliche Uhrzeit:

Effektive Schlafzeit (Stunden) pro Nacht:

Kreuzen Sie bitte für jede der folgenden Fragen die für Sie zutreffende Antwort an. Beantworten Sie bitte alle Fragen.

\section{Wie oft haben Sie während der letzten vier Wochen schlecht geschlafen, ...}

a) ... weil Sie nicht innerhalb von 30 Minuten einschlafen konnten?

b) ... weil Sie mitten in der Nacht oder früh morgens aufgewacht sind?

c) ... weil Sie aufstehen mußten, um zur Toilette zu gehen?
Während der letzten vier Wochen gar nicht

O Weniger als einmal pro Woche

E Einmal oder zweimal pro Woche

Dreimal oder häufiger pro Woche

Während der letzten vier Wochen gar nicht

$O$ Weniger als einmal pro Woche

O Einmal oder zweimal pro Woche

Dreimal oder häufiger pro Woche

Während der letzten vier Wochen gar nicht

O Weniger als einmal pro Woche

O Einmal oder zweimal pro Woche

Dreimal oder häufiger pro Woche 
d) ... weil Sie Beschwerden beim Atmen hatten?

e) ... weil Sie husten mußten oder laut geschnarcht haben?

f) ... weil Ihnen zu kalt war?

g) ... weil Ihnen zu warm war?

h) ... weil Sie schlecht geträumt hatten?

i) ... weil Sie Schmerzen hatten?

j) ... aus anderen Gründen?

Bitte beschreiben:

\section{Wie würden Sie insgesamt die Qualität Ihres Schlafes während der letzten vier Wochen beurteilen?}

Sehr gut

O Ziemlich gut

O Ziemlich schlecht

S Sehr schlecht 
7. Wie oft haben Sie während der letzten vier Wochen Schlafmittel eingenommen (vom Arzt verschriebene oder frei verkäufliche)?

8. Wie oft hatten Sie während der letzten vier Wochen Schwierigkeiten wachzubleiben, etwa beim Autofahren, beim Essen oder bei gesellschaftlichen Anlässen?

9. Hatten Sie während der letzten vier Wochen Probleme, mit genügend Schwung die üblichen Alltagsaufgaben zu erledigen?
Während der letzten vier Wochen gar nicht

Weniger als einmal pro Woche

O Einmal oder zweimal pro Woche

Dreimal oder häufiger pro Woche

Während der letzten vier Wochen gar nich

$O$ Weniger als einmal pro Woche

O Einmal oder zweimal pro Woche

Dreimal oder häufiger pro Woche

Keine Probleme

O Kaum Probleme

Otwas Probleme

O Große Probleme

10. Schlafen Sie allein in Ihrem Zimmer?

O Ja

O Ja, aber ein Partner/Mitbewohner schläft in einem anderen Zimmer

Nein, der Partner schläft im selben Zimmer, aber nicht im selben Bett

O Nein, der Partner schläft im selben Bett

Falls Sie einen Mitbewohner / Partner haben, fragen Sie sie/ihn bitte, ob und wie oft er/sie bei Innen folgendes bemerkt hat.

a) Lautes Schnarchen

b) Lange Atempausen während des Schlafes

c) Zucken oder ruckartige Bewegungen der Beine während des Schlafes
Während der letzten vier Wochen gar nicht

Weniger als einmal pro Woche

O Einmal oder zweimal pro Woche

Dreimal oder häufiger pro Woche

Während der letzten vier Wochen gar nicht

O Weniger als einmal pro Woche

O Einmal oder zweimal pro Woche

Dreimal oder häufiger pro Woche

Während der letzten vier Wochen gar nicht

Weniger als einmal pro Woche

O Einmal oder zweimal pro Woche

Dreimal oder häufiger pro Woche 
d) Nächtliche Phasen von Verwirrung oder Desorientierung während des Schlafes

e) Oder andere Formen von Unruhe während des Schlafes
Während der letzten vier Wochen gar nicht

Weniger als einmal pro Woche

O Einmal oder zweimal pro Woche

Dreimal oder häufiger pro Woche

Machen Sie bitte noch folgende Angaben zu Ihrer Person:

Alter:

Jahre

Geschlecht: (weiblich
Körpergröße:

Beruf:

O Rentner(in) ( Schüler/Student(in) (Arbeiter(in)
Gewicht:

( selbständig

( Angestellte( $r$ )

(arbeitslos/ Hausfrau(mann) 
7.10. Anhang 10: Clinical Global Impression Scale, CGI (Guy 1976)

\section{Severity of illness}

Considering your total clinical experience with this particular population of migraine patients, how ill is the subject at this time?

Not assessed 0

Normal, not ill at all 1

Borderline ill 2

Mildly ill 3

Moderately ill 4

Markedly ill 5

Severely ill 6

Among the most extremely ill subjects $\quad 7$ 


\section{Verzeichnis der Tabellen}

Tabelle 1: Wichtige Differenzialdiagnosen der Migräne und notwendige Diagnostik 4

Tabelle 2: Zusammenfassung der wichtigsten Empfehlungen zur Akuttherapie und Prophylaxe der Migräne. 9

Tabelle 3: Analgetika und nicht steroidale Antirheumatika (NSAR) in der Behandlung der akuten Migräneattacke. 10

Tabelle 4: Antiemetika in der Migränetherapie. 10

Tabelle 5: Therapie der akuten Migräneattacke mit 5-HT1B/1D-Agonisten (Reihenfolge nach dem Jahr der Zulassung).

Tabelle 6: Therapie der akuten Migräneattacke mit Mutterkornalkaloiden 12

Tabelle 7: Substanzen zur Migräneprophylaxe mit guter Evidenzlage (Substanzen der ersten Wahl zur Migräneprophylaxe).

Tabelle 8: Substanzen zur Migräneprophylaxe mit weniger guter Evidenzlage (Substanzen der zweiten

Wahl zur Migräneprophylaxe). 17

Tabelle 9: Übersicht über nicht medikamentöse Therapieverfahren in der Migräneprophylaxe 19

Tabelle 10: Einschluss- und Ausschlusskriterien 35

Tabelle 11: Gesundheitskonzepte, Itemzahl und Anzahl der Stufen sowie Inhalt der acht SF-36 Skalen und des Items zur Veränderung des Gesundheitszustandes

Tabelle 12: Randomisierungsprotokoll (Patienten von 1 bis 30 als Beispiel)

Tabelle 13: Demographische Daten der Probanden im Gruppenvergleich zur Baseline. Angaben in

Prozent oder im arithmetischen Mittelwert (Standardabweichung in Klammern)

Tabelle 14: Ergebnisse der Hauptparameter zur Baseline, angegeben als arithmetischer Mittelwert

(Standardabweichung in Klammern)

Tabelle 15: Begleitsymptome zur Baseline, angegeben als arithmetischer Mittelwert

(Standardabweichung in Klammern)

Tabelle 16: Subskalenwerte des SF-36 zur Baseline, angegeben als arithmetischer Mittelwert

(Standardabweichung in Klammern) 48 
Tabelle 17: Psychometrische Parameter zur Baseline, angegeben als arithmetischer Mittelwert (Standardabweichung in Klammern)

Tabelle 18: Hauptparameter im Verlauf der Untersuchung in der LD-Gruppe, angegeben als arithmetischer Mittelwert (Standardabweichung in Klammern)

Tabelle 19: Begleitsymptome und Schmerzintensität im Verlauf der Untersuchung in der LD-Gruppe, angegeben als arithmetischer Mittelwert (Standardabweichung in Klammern) 50

Tabelle 20: Psychometrische Parameter im Verlauf der Untersuchung in der LD-Gruppe, angegeben als arithmetischer Mittelwert (Standardabweichung in Klammern)

Tabelle 21:Veränderung der Subskalen des SF-36 im Verlauf der Untersuchung in der LD-Gruppe, angegeben als arithmetischer Mittelwert (Standardabweichung in Klammern) im Vergleich mit einer Normstichprobe.

Tabelle 22: Hauptparameter im Verlauf der Untersuchung in der KM-Gruppe, angegeben als arithmetischer Mittelwert (Standardabweichung in Klammern)

Tabelle 23: Begleitsymptome und Schmerzintensität im Verlauf der Untersuchung in der KM-Gruppe, angegeben als arithmetischer Mittelwert (Standardabweichung in Klammern)

Tabelle 24: Psychometrische Parameter im Verlauf der Untersuchung in der KM-Gruppe, angegeben als arithmetischer Mittelwert (Standardabweichung in Klammern)

Tabelle 25: Veränderung der Subskalen des SF-36 im Verlauf der Untersuchung in der KM-Gruppe, angegeben als arithmetischer Mittelwert (Standardabweichung in Klammern) im Vergleich mit einer Normstichprobe ( ${ }^{*}$ nach Bullinger und Kirchberger 1998, S 33).

Tabelle 26: Hauptparameter im Verlauf der Untersuchung in der WG, angegeben als arithmetischer Mittelwert (Standardabweichung in Klammern)

Tabelle 27: Begleitsymptome und Schmerzintensität im Verlauf der Untersuchung in der WG, angegeben als arithmetischer Mittelwert (Standardabweichung in Klammern)

Tabelle 28: Psychometrische Parameter im Verlauf der Untersuchung in der WG-Gruppe, angegeben als arithmetischer Mittelwert (Standardabweichung in Klammern)

Tabelle 29: Subskalen des SF-36 im Verlauf der Untersuchung in der WG-Gruppe, angegeben als arithmetischer Mittelwert (Standardabweichung in Klammern) im Vergleich mit einer Normstichprobe 
Tabelle 30: Kopfschmerztage aller Gruppen zu allen Zeitpunkten, angegeben als arithmetischer Mittelwert (Standardabweichung in Klammern) 62

Tabelle 31: Migränetage aller Gruppen zu allen Zeitpunkten, angegeben als arithmetischer Mittelwert

(Standardabweichung in Klammern) .....

Tabelle 32: Migräneattacken aller Gruppen zu allen Zeitpunkten, angegeben als arithmetischer Mittelwert (Standardabweichung in Klammern) 64

Tabelle 33: Eingenommene Akutmedikation aller Gruppen zu allen Zeitpunkten, angegeben als arithmetischer Mittelwert (Standardabweichung in Klammern). 65

Tabelle 34: Unterschied der Responderraten und der Differenz der Migräneattacken, Migränetage und Kopfschmerztage in allen drei Gruppen zwischen Baseline und Beobachtungsende. Angaben in Prozent oder im arithmetischen Mittelwert (Standardabweichung in Klammern)66

Tabelle 35: Ergebnisse der sekundären Endpunkte (Unterschied zwischen Baseline und Behandlungsende). Angaben in Prozent oder im arithmetischen Mittelwert (Standardabweichung in Klammern)

Tabelle 36: Schmerzintensität aller Gruppen zu allen Zeitpunkten, angegeben als arithmetischer Mittelwert (Standardabweichung in Klammern) 68

Tabelle 37: Ergebnisse des IBK im Verlauf der Untersuchung in allen drei Gruppen, angegeben als arithmetischer Mittelwert (Standardabweichung in Klammern)

Tabelle 38: Ergebnisse des Midas in Verlauf der Untersuchung in allen drei Gruppen, angegeben als arithmetischer Mittelwert (Standardabweichung in Klammern)

Tabelle 39: Unterschied der Differenz des Midas in allen drei Gruppen zwischen Baseline und Behandlungsende (sekundärer Endpunkt) sowie zwischen Baseline und Nachbeobachtung (primärer Endpunkt). Angaben im arithmetischen Mittelwert (Standardabweichung in Klammern).

Tabelle 40: Ergebnisse der CES-D im Verlauf der Untersuchung in allen drei Gruppen, angegeben als arithmetischer Mittelwert (Standardabweichung in Klammern).

Tabelle 41: Ergebnisse des SF-36 im Verlauf der Untersuchung in allen drei Gruppen (körperliche Summenskala), angegeben in transformierten Werten.

Tabelle 42: Ergebnisse des SF-36 im Verlauf der Untersuchung in allen drei Gruppen (psychische Summenskala), angegeben in transformierten Werten. 
Tabelle 43: Ergebnisse aller Subskalen des SF-36 in allen drei Gruppen zum Zeitpunkt der Nachbeobachtung (T4), angegeben als arithmetischer Mittelwert (Standardabweichung in Klammern) 75

Tabelle 44: Ergebnisse aller Subskalen des SF-36 in allen drei Gruppen zum Behandlungsende (T3), angegeben als arithmetischer Mittelwert (Standardabweichung in Klammern) 75

Tabelle 45: PSQI im Verlauf der Untersuchung in allen drei Gruppen, angegeben als arithmetischer Mittelwert (Standardabweichung in Klammern) 77

Tabelle 46: Ergebnisse des CGI im Verlauf der Untersuchung in allen drei Gruppen, angegeben als arithmetischer Mittelwert (Standardabweichung in Klammern) 79 


\section{Verzeichnis der Abbildungen}

Abbildung 1: $\quad$ Studiendesign für Behandlungsgruppen und Kontrollgruppe in

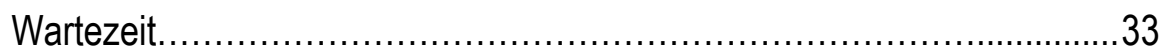

Abbildung 2: $\quad$ Studiendesign für Kontrollgruppe in Intervention.............................. 33

Abbildung 3: $\quad$ Zeitlicher Ablauf der Datenerhebung (Hauptteil dieser Studie) ................ 36

Abbildung 4: $\quad$ Zeitlicher Ablauf der Datenerhebung bei der Kontrollgruppe während der Intervention.

Abbildung 5: $\quad$ Prozentuale Darstellung der Differenz der Hauptparameter in der LDGruppe.

Abbildung 6: $\quad$ Prozentuale Differenz der Begleitsymptome und Schmerzintensität im Verlauf der Untersuchung in der LD-Gruppe

Abbildung 7: $\quad$ Subskalen des SF-36, angebenen als arithmetischer Mittelwert in der

LD-Gruppe

Abbildung 8: $\quad$ Prozentuale Differenz der psychometrischen Parameter in Verlauf der

Untersuchung in der LD-Gruppe. 53

Abbildung 9: $\quad$ Prozentuale Darstellung der Differenz der Hauptparameter in der KM Gruppe 54

Abbildung 10: $\quad$ Prozentuale Differenz der Begleitsymptome und der Schmerzintensität im Verlauf der Untersuchung in der KM-Gruppe

Abbildung 11: $\quad$ Prozentuale Differenz der psychometrischen Parameter im Verlauf der Untersuchung in der KM-Gruppe

Abbildung 12: $\quad$ Subskalen des SF-36, angegeben als arithmetischer Mittelwert in der KM-Gruppe

Abbildung 13: $\quad$ Prozentuale Darstellung der Differenz der Hauptparameter in der Wartegruppe .58

Abbildung 14: $\quad$ Prozentuale Differenz der Begleitsymptome und der Schmerzintensität im Verlauf der Untersuchung in der WG-Gruppe 
Abbildung 15: $\quad$ Prozentuale Differenz der psychometrischen Parameter im Verlauf der Untersuchung in der Wartegruppe.

Abbildung 16: $\quad$ Subskalen des SF-36 in der Wartegruppe, angegeben als arithmetischer Mittelwert

Abbildung 17: $\quad$ Darstellung der Kopfschmerztage aller Gruppen zu T3 und T4 im Vergleich zur Baseline, angegeben als arithmetischer Mittelwert.

Abbildung 18: Differenz der Migränetage aller Gruppen zum primären Endpunkt, angegeben als Mittelwert, minimale und maximale Differenzwerte.

Abbildung 19: $\quad$ Darstellung der Migränetage aller Gruppen zu T3 und T4 im Vergleich zur Baseline, angegeben als arithmetischer Mittelwert

Abbildung 20: Differenz der Migräneattacken aller Gruppen zum primären Endpunkt, angegeben als Mittelwert, minimale und maximale Differenzwerte

Abbildung 21: $\quad$ Darstellung der Migräneattacken aller Gruppen zu T3 und T4 im Vergleich zur Baseline, angegeben als arithmetischer Mittelwert

Abbildung 22: $\quad$ Darstellung der Schmerzmedikation aller Gruppen zu T3 und T4 im Vergleich zur Baseline, angegeben als arithmetischer Mittelwert

Abbildung 23: Differenz der Migräneattacken zum sekundären Endpunkt, angegeben als arithmischer Mittelwert, minimale und maximale Differnzwerte.

Abbildung 24: $\quad$ Darstellung der Schmerzintensität aller Gruppen, angegeben als arithmetischer Mittelwert

Abbildung 25: $\quad$ IBK aller Gruppen im Vergleich, angegeben als arithmetischer Mittelwert.

Abbildung 26: $\quad$ Prozentuale Differenz des IBK aller Gruppen im Vergleich zum primären und sekundären Endpunkt

Abbildung 27: $\quad$ Gesamtscore des Midas aller Gruppen im Vergleich, angegeben als arithmetischer Mittelwert.

Abbildung 28: $\quad$ Prozentuale Differenz des Midas aller Gruppen zum primären und sekundären Endpunkt 
Abbildung 29: $\quad$ Gesamtscore der CES-D aller Gruppen im Vergleich, angegeben als arithmetischer Mittelwert

Abbildung 30: $\quad$ Prozentuale Differenz der CES-D aller Gruppen zum primären (T4) und sekundären Endpunkt (T3)

Abbildung 31: $\quad$ Körperliche Summenskala des SF-36 aller Gruppen im Vergleich........... 73

Abbildung 32: $\quad$ Psychische Summenskala des SF-36 aller Gruppen im Vergleich............74

Abbildung 33: $\quad$ Veränderung der körperlichen und psychischen Summenskala des SF36 zum primären und sekundären Endpunkt, angegeben als prozentuale Differenz.

Abbildung 34: $\quad$ Alle Subskalen des SF-36 aller Gruppen zum primären Endpunkt, angegeben als prozentuale Differenz zur Baseline

Abbildung 35: $\quad$ Alle Subskalen des SF-36 aller Gruppen zum sekundären Endpunkt, angegeben als prozentuale Differenz zur Baseline

Abbildung 36: $\quad$ Gesamtscore des PSQI aller Gruppen im Vergleich, angegeben als arithmetischer Mittelwert

Abbildung 37: $\quad$ Prozentuale Differenz des PSQI aller Gruppen zum primären (T4) und sekundären (T3) Endpunkt

Abbildung 38: $\quad$ Gesamtscore des CGI aller Gruppen im Vergleich, angegeben als arithmetischer Mittelwert

Abbildung 39: $\quad$ Prozentuale Differenz des CGI aller Gruppen zum primären und sekundären Endpunkt

Abbildung 40: $\quad$ Körperliche Summenskala des SF-36 zu T3 und T4 im Vergleich mit einer Normstichprobe

Abbildung 41: $\quad$ Psychische Summenskala der SF-36 zu T3 und T4 im Vergleich mit einer Normstichprobe 


\section{Literaturverzeichnis}

Andrasik F (1996): Behavioral management of migraine. Biomed Pharmacother 50, 52-57

Andrasik F (2004): Behavioral treatment of migraine: Current Status and future directions. Expert Rev Neurother $\underline{4}, 403-413$.

Andresen EM, Malmgren JA, Carter WB, Patrick DL (1994): Screening for depression in well older adults: Evaluation of a short form of the CES-D. Am J Prev Med 10, 77-84

Bauer B, Evers S, Gralow I, Husstedt IW (1999): Psychosoziale Beeinträchtigung durch Kopfschmerzen: Evaluation des Inventars zur Beeinträchtigung durch Kopfschmerzen (IBK). Nervenarzt $\underline{70}, 522-529$

Biondi DM (2005): Physical treatment of headache: a structured review. Headache $\underline{45}, 738-746$

Bringezu G, Schreiner O: Lehrbuch der Entstauungstherapie 2. Springer-Verlag GmbH, Berlin und Heidelberg 2001

Bullinger M, Kirchberger I: SF-36 Fragebogen zum Gesundheitszustand, Hogrefe-Verlag GmbH \& Co.KG, Göttingen 1998

Busch V, Gaul C (2008): Sport bei Migräne: Übersicht und Diskussion sowie Implikationen für zukünftige Studien. Schmerz $\underline{22}, 137-147$

Buysse DJ, Reynolds CF, Monk TH, Berman SB, Kupfer DJ (1989): The Pittsburgh Sleep Quality Index: a new instrument for psychiatric practice and research. Psychiatry Res $\underline{28}, 193-213$

Carano A, Siciliani G (1996): Effects of continuous and intermittent forces on human fibroblasts in vitro. Eur J Orthod 18, 19-26

Chadaide Z, Arlt S, Antal A, Nitsche MA, Lang N, Paulus W (2007): Transcranial direct current stimulation reveals inhibitory deficiency in migraine. Cephalalgia $\underline{27}, 833-839$ 
Delepine L, Aubineau P (1997): Plasma protein extravasation induced in the rat dura mater by stimulation of the parasympathetic sphenopalatine ganglion. Exp Neurol $\underline{147}, 389-400$

Diener HC, Pfaffenrath V, Limmroth V, Brune K, Fritsche G, Evers S, Kropp P, May A, Straube A:

Therapie der Migräneattacke und Migräneprophylaxe: Gemeinsame Leitlinie der Deutschen

Gesellschaft für Neurologie und der Deutschen Migräne- und Kopfschmerzgesellschaft; In: Leitlinien für Diagnostik und Therapie in der Neurologie 2005; hrsg. v. Kommission Leitlinien der Deutschen

Gesellschaft für Neurologie. 3. Auflage; Georg Thieme Verlag, Stuttgart 2005, 494-508

Diener HC, Gendolla A, Katsarava Z, Slomke MA (2006): Neues bei Kopfschmerzen. Aktuell Neurol $\underline{33}$, $480-484$

Dimitriadou V, Buzzi MG, Moskowitz MA, Theoharides TC (1991): Trigeminal sensory fiber stimulation induces morphological changes reflecting secretion in rat dura mater mast cells. Neuroscience $44,97-$ 112

Ebersberger A (2002): Pathophysiologie der Migräne. Anaesthesist $\underline{51,661-667}$

Endres HG, Diener HG, Maier C, Böwing G, Trampisch HJ, Zenz M (2007): Akupunktur bei chronischen Kopfschmerzen. Dtsch Ärztebl 104, 114-122

Ernst E (1999): Homeopathic prophylaxis of headache and migraine: A systematic review. J Pain Symptom Manage $\underline{18}, 353-357$

Evans RW, Lipton RB, Silberstein SD (2005): The prevalence of migraine subjects disability and quality of life and acute migraine management. Pain $\underline{118}, 319-326$

Evers S, Göbel H (2004): Die deutschsprachige Übersetzung der Kopfschmerzklassifikation der IHS. 2. Auflage; Internetbasierte Version. www.ihs-classification.org/de/01_einleitung/

Evers S, Afra J, Frese A, Goadsby PJ, Linde M, May A, Sandor PS (2006): EFNS guideline on the drug treatment of migraine. Eur J Neurol $\underline{13}, 560-572$ 
Evers S, May A, Fritsche G, Kropp P, Lampl C,Limmroth V, Malzacher V, Sander P, Straube A, Diener HC (2008): Akuttherapie und Prophylaxe der Migräne: Leitlinien der Deutschen Migräne- und Kopfschmerzgesellschaft und der deutschen Gesellschaft für Neurologie. Nervenheilkunde $\underline{10}$, 933-949

Field TM (1998): Massage therapy effects. Am Psychol $\underline{53}$, 1270-1281

Field T, Morrow C, Valdeon C, Larson S, Kuhn C, Schanberg S (1992): Massage reduces anxiety in child and adolescent psychiatric patients. J Am Acad Child Adolesc Psychiatry 31, 125-131

Goadsby PJ (1993): The trigeminovascular system and migraine: studies characterizing cerebravascular and neuropeptide changes seen in humans and cats. Ann Neurol $\underline{33}, 48-56$

Goadsby PJ, Edvinsson L, Ekman R (1988): Release of vasoactive peptides in the extracerebral circulation of humans and the cats during activation of the trigeminovascular system. Ann Neurol $\underline{23}$, 193-196

Grazzi L, Andrasik F, D’Amico D, Leone M, Usai S, Kass S, Bussone G (2002): Behavioral and pharmacologic treatment of transformed migraine with analgesic overuse: outcome at 3 years. Headache $\underline{42}, 483-490$

Guy W: ECDEU Assessment Manual for Psychopharmacology. US Department of Health, Education, and Welfare. Washington, DC 1976

Hautzinger M, Bailer M: Allgemeine Depressionsskala (ADS). Beltz Verlag, Weinheim 1993

Headache Classification Subcommittee of the International Headache Society (2004): The International classification of headache disorders, 2nd edition. Cephalalgia 24, 1-160

Heisel J: Physikalische Medizin (Praxiswissen Halte- und Bewegungsorgane).Thieme Verlag KG, Stuttgart 2005 
Hermann C, Kim M, Blanchard EB (1995): Behavioural and prophylactic intervention studies of pediatric migraine: an exploratory meta-analysis. Pain $\underline{60}, 239-256$

Hernandez-Reif M, Dieter J, Field T (1998): Migraine headaches are reduced by massage therapy. Int J Neurosci $\underline{96}, 1-11$

Ho TW, Mannix LK, Fan X, Assaid C, Furtek C, Jones CJ, Lines CR, Rapoport AM (2008): Randomized controlled trial of an oral CGRP receptor antagonist, MK-0974, in acute treatment of migraine. Neurology $\underline{70}, 1304-1312$

Holroyd KA, Penzien DB (1990): Pharmacological versus non-pharmacological prophylaxis of recurrent migraine headache: a meta-analytic review of clinical trials. Pain $\underline{42}, 1-13$

Holroyd KA, France JL, Cordingley GE, Rokicki LA, Kvaal SA, Lipchik GL, McCool HR (1995): Enhancing the effectiveness of relaxation-thermal biofeedback training with propranolol hydrochloride. $J$ Consult Clin Psychol 63, 327-330

Jacobson GP, Ramadan NM, Aggarwal SK, Newman CW (1994): The Henry Ford Hospital Headache disability Inventory (HDI). Neurology $\underline{44}, 837-842$

Kloster BC: Massage. 2. Auflage; Springer-Verlag GmbH, Berlin 2006

Krahl J (2005): Die Effektivität der physiotherapeutischen Behandlung von Migränepatienten mit Manueller Lymphdrainage anhand von drei Einzelfallstudien. Physioscience 1, 52-57

Kurth T, Slomke MA, Kase CS, Cook NR, Lee IM, Gaziano JM, Diener HC, Buring JE (2005): Migraine, headache, and the risk of stroke in women. Neurology $\underline{64}, 1020-1026$

Lantéri-Mint M, Radat F, Chautard MH, Lucas C (2005): Anxiety and depression associated with migraine: Influence on migraine subject's disability and quality of life, and acute migraine management. Pain 118, 319-326 
Lawler SP, Cameron LD (2006): A randomized, controlled trial of massage as a treatment for migraine. Ann Behav Med 32, 50-59

Leinisch E, Evers S, Kaempfe N, Kraemer C, Sostak P, Jürgens T, Straube A, May A (2005): Evaluation of the efficacy of intravenous acetaminophen in the treatment of acute migraine attacks: a double-blind, placebo-controlled parallel group multicenter study. Pain $\underline{117}, 196-400$

Limmroth V, Diener HC (2003): Neues zu Pathophysiologie und Therapie der Migräne. Notfallmedizin $\underline{29}, 32-41$

Limmroth V, Katsarava Z, Liedert B, Guehring H, Schmitz K, Diener HC, Michel MC (2001): A new in vivo rat model for CGRP release following the activation of trigeminal vascular system. Pain $\underline{92}, 101-106$

Longo C, Rizzo R, Inzitari MT, Scumaci G, Caroleo S, locco M (2006): Chronic tension-type headache non-conventional therapy with manual lymphatic drainage. Recenti Prog Med $\underline{97}$, 462-465

Markowitz S, Saito K, Moskowitz MA (1987): Neurologically mediated leakage of plasma protein occurs from blood vessels in dura mater but not brain. J Neurosci $\underline{7}, 4129-4136$

May A (2006): Diagnostik und moderne Therapie der Migräne. Dtsch Ärztebl 17, 1157-1166

May A, Ophoff RA, Terwindt GM, Urbam C, Eijk R van, Haan J, Diener HC, Lindhout D, Frants RR, Sandkuyl LA, Ferrari MD (1995): Familial hemiplegic migraine locus on chromosome 19p13 is involved in common forms of migraine with and without aura. Hum Genet $\underline{96}, 604-608$

Müller-Oerlinghausen B, Berg C (2003): Wirkungen von "therapeutischer Berührung" bei Depression und anderen psychiatrischen Störungen. Dtsch Z Klini Forschung 1/2, 56-58

Müller-Oerlinghausen B, Berg C, Droll W (2004): Wirkung einer"slow stroke" Massage als komplementäre Therapie bei stationären depressiven Patienten. Dtsch Med Wochenschr $\underline{129}, 1363-$ 1368 
Narin SO, Pinar L, Erbas D, Oztürk V, Idimin F (2003): The effects of exercise and exercise-related changes in blood nitric oxide level on migraine headache. Clin Rehabil 17, 624-630

Nestoriuc Y, Martin A (2007): Efficacy of biofeedback for migraine: a meta-analysis. Pain $\underline{128}$, 111-127

Nyholt DR, Lea RA, Goadsby PJ, Brimage PJ, Griffiths LR (1998): Familial typical migraine: linkage to chromosome 19p13 and evidence for genetic heterogeneity. Neurology $\underline{50}$, 1428-1432

Olesen J, Friberg L, Olsen TS, Iversen HK, Lassen NA, Andersen AR, Karle A (1990):Timing and topography of cerebral blood flow, aura, and headache during migraine attacks. Ann Neurol $\underline{28}, 791-798$

Olesen J, Diener HC, Husstedt IW, Goadsby PJ, Hall D, Meier U, Pollentier S, Lesko LM (2004): BIBN 4096 BS Clinical Proof of Concept Study Group. Cacitonin gene-releted peptide receptor antagonist BIBN 4096 BS for the acute treatment of migraine. N Engl J Med $\underline{350}, 1104-1110$

Ophoff RA, Terwindt GM, Vergouwe MN, van Eijk R,Hoffman SMG, Lamerdin JE, Mohreweiser HW, Bulman DE, Ferrari M, Haan J, Lindhaout D, van Ommen GB,Hofker MH, Ferrari MD, Frants RR (1997): Familial hemiplegic migraine and episodic ataxia type-2 are caused by mutations in the $\mathrm{Ca} 2+$ channel gene CACNL1A4. Cell $\underline{87}, 543-552$

Penzien DB, Andrasik F, Freidenberg BM ,Houle TT, Lake AE 3rd, Lipchik GL, Holroyd KA, Lipton RB, McCrory DC, Nash JM, Nicholson RA, Powers SW, Rains JC, Wittrock DA (2005): Guidelines for trials of behavioral treatments for reccurent headache, first edition: American Headache Society Behavioral Clinical Trials Workgroup. Headache 47 (Suppl 2), 110-132

Peroutka SJ (2005): Neurogenic inflamation and migraine: Implications for therapeutics. Mol Interv $\underline{5}$, 304-311

Puustjarvi K, Airaksinen O, Pontinen P (1990): The effects of massage in patients with chronic tension headache. Acupunct Electrother Res $\underline{15}, 159-162$

Quinn C, Chandler C, Moraska A (2002): Massage therapy and frequency of chronic tension headaches. Am J Public Health $\underline{92}, 1657-1661$ 
Randolf LS (1977): The CES-D scale: a self-report depression scale for research in the general population. Appl psych Meas. 1 , 385-401

Reid GJ, McGrath PJ (1996): Psychological treatments for migraine. Biomed Pharmacother $\underline{50}$, 58-63

Sandor PS, Di Clemente L, Coppola G, Saenger U, Fumal A, Magis D, Seidel L, Agosti RM, Schoenen J (2005): Efficacy of coenzyme Q10 in migraine prophylaxis: a randomized controlled trial. Neurology $\underline{64}$, 713-715

Stanley MI, Berger RJ, Zucarello M, Keller TJ (1993): Serotonin (5-HT) fibers of the rat dura mater: 5HT- positive but not authentic serotoninergic, tryptophane hydroxylase-like fibers. Neurosci Lett $\underline{162}, 89$ 92

Stewart WF, Lipton RB, Whyte J, Dowson A, Kolodner K, Liberman JN, Sawyer J (1999): An international study to assess reliability of the Migraine Disability Assessment (Midas) score. Neurology $\underline{53}, 988-994$

Straumsheim P, Borchgrevink C, Mowinckel P, Kierulf H, Hafslund O (2000): Homeopathic treatment of migraine: a double blind, placebo controlled trial of 68 patients. Br Homeopathol J $\underline{89}, 4-7$

Thomson LL, Olesen J (1995): The autonomic nervous system and regulation of arterial tone in migraine. Clin Auton Res $\underline{5}, 243-250$

Walach H, Klöpfer D, König M, Ludwig E: Massage: Wirksamkeit, Wirkung und psychologische Effekte. Literaturbericht, Expertise und Pilotstudie. Haug Verlag, Heidelberg 1995

Walach H, Haeusler W, Lowes T, Mussbach D, Schamell U, Springer W, Stritzl G, Gaus W, Haag G (1997): Classical homeopathic treatment of chronic headaches. Cephalalgia 117, 119-126

Ware JE, Snow KK, Kosinski M, Gandek B: SF-36 Health Survey manual and interpretation guide. The Health Institute, New England Medical Center, Boston MA 1993 
Weiller C, May A, Limmroth V (1995): Brainstem activation in spontaneous human migraine attacks. Nat Med 1, 658-660

Weinberg R, Jackson A, Kolodny K (1988): The relationship of massage and exercise to mood enhancement. The Sport Psychologist 2, 2002-2011

Werner GT, Bieger WP, Blum B, Hentschel HD, Huber C, Penz M (1997): Wirkungen einer Serie von Ganzkörpermassage auf zahlreiche Parameter des Immunsystems. Phys Rehabil Kur Med $\underline{7}, 51-54$

Whitmarsh TE, Coleston-Shields DM, Steiner TH (1997): Double-blind randomized placebo-controlled study of homoeopathic prophylaxis of migraine. Cephalalgia 17, 600-604

Winner P, Wasiewski W, Gladstein J, Linder S (1997): Multicenter prospective evaluation of proposed pediatric migraine revisions to the IHS criteria. Pediatric Headache Committee of the American Association of the Study of Headache. Headache $\underline{37}, 545-548$ 


\section{Abkürzungsverzeichnis}

ASS Acetylsalicylsäure

CCT Kraniale Computertomographie

CES-D Center for Epidemiologic Studies Depression Scale

CGI Clinical Global Impression

CGRP calcitonin gene related peptide

DMKG Deutsche Migräne- und Kopfschmerzgesellschaft

EEG Elektroenzephalographie

EFNS European Federation of Neurological Societies

G1 Gruppe 1= Lymphdrainage Gruppe

G2 Gruppe 2= klassische Massage Gruppe

G3 Kontrollgruppe $=$ Wartegruppe

G3a Wartegruppe mit später Lymphdrainage

G3b Wartegruppe mit später klassischer Massage

IBK Inventar zur Beeinträchtigung durch Kopfschmerzen

IHS International Headache Society

KKB Kalziumkanalblocker

KM Klassische Massage

KVT kognitive Verhaltenstherapie

LD Lymphdrainage

Midas Migraine disability assessment

MLD Manuelle Lymphdrainage

NMR nuclear magnetic resonance; Kernspinresonanz

NO Stickstoffoxid

NSAR Nicht-steroidale Antirheumatika 
PAG Periaquäduktales Grau

PMR progressive Muskelrelaxation

PSQI Pittsburgh Sleep Quality Index

SF-36 Lebensqualitätsfragebogen

SF(kö) SF-36 (körperliche Summenskala)

SF(psy) SF-36 (psychische Summenskala)

T0 Zeitpunkt zur Aufnahme

T1 Zeitpunkt 4 Wochen nach Aufnahme = Baseline

T2 Zeitpunkt 8 Wochen nach Aufnahme $=4$ Wochen nach Beginn der Behandlung

T3 Zeitpunkt 12 Wochen nach Aufnahme $=8$ Wochen nach Beginn der Behandlung = Behandlungsende $=$ Sekundärer Endpunkt

T4 Zeitpunkt 16 Wochen nach Aufnahme $=4$ Wochen nach Ende der Behandlung = Beobachtungsphase $=4$ Wochen nach Behandlungsende $=$ Primärer Endpunkt

TENS Transkutane Elektrische Nervenstimulation 


\section{Danksagung}

Für die freundliche Überlassung des Themas und die stete Unterstützung bei dieser Arbeit danke ich herzlich Frau Prof. Dr. med. Happe.

Herrn Dr. med. Siegert möchte ich dafür danken, dass er es mir ermöglicht hat, diese Untersuchung an dem Institut für Physikalische und Rehabilitative Medizin durchführen zu können.

Herrn Dr. med. Peikert danke ich für die Hilfe bei der Auswahl bzw. Überweisung der von ihm betreuen Patientinnen und Patienten.

Mein besonderer Dank gilt allen Masseurinnen und Masseuren des Institutes für Physikalische und Rehabilitative Medizin des Klinikums Bremen-Ost für die allzeit geduldige Unterstützung in der Organisation und Durchführung der unterschiedlichen Therapien.

Den Patientinnen und Patienten, die an dieser Studie teilgenommen haben, danke ich herzlich. 


\section{Lebenslauf}

Am 11.04.1977 wurde ich in Stef, Algerien geboren.

Von 1982 bis 1991 besuchte ich die Grund- und Mittelschule in „Rsas“; Stadtbezirk Al-Suieda in Syrien, anschließend das Gymnasium „Shakib Arsalan“ in derselben Stadt. Dort erwarb ich am 19.07.1994 die allgemeine Hochschulreife.

Vom Wintersemester 1994 bis zum Sommersemester 2000 studierte ich an der Fakultät für Humanmedizin der Universität Damaskus. Nach Kenntnisnahme des Ergebnisses der Abschlussprüfung (Juli 2000) wurde mir am 29.08.2000 der akademische Grad „Licence Doktor der Humanmedizin" verliehen.

Im Jahr 2001 absolvierte ich die „Basic- und Clinical Science Examination - United States Medical Licening (USMLE-Step1 and 2).

Von Dezember 2001 bis Mai 2003 war ich als Arzt im Praktikum in der Abteilung für Innere Medizin und anschließend in der Abteilung für Hals-Nasen-Ohrenheilkunde der Universitätskliniken in Damaskus tätig.

Ab Mai 2003 war ich als Vollzeit-Fakultätsassistent an der medizinischen Fakultät, Universität Damaskus, Abteilung für Innere Medizin, Institut für Physikalische Medizin und Rehabilitation tätig.

In Juli 2004 erhielt ich ein Stipendium der Universität Damaskus zum Erwerb der Facharztqualifikation im Gebiet Physikalische und Rehabilitative Medizin.

Vom 01.01.2005 bis zum 30.06.2010 war ich als Arzt in Weiterbildung für das Fachgebiet Physikalische und Rehabilitative Medizin im Klinikum Bremen-Ost in Bremen tätig.

Das Chirurgische Jahr habe ich im Jahr 2005 in der Klinik für Allgemein-, Viszeral und Unfallchirurgie des Klinikums Bremen-Ost absolviert, anschließend vom 01.01.2006 bis 31.12.2006 das internistische Jahr in den Kliniken für Innere Medizin, Geriatrie und Pneumologie des Klinikums Bremen-Ost.

Ab dem Jahr 2007 erfolgte die Weiterbildung für Physikalische und Rehabilitative Medizin im Institut für Physikalische und Rehabilitative Medizin, in der Klinik für Geriatrie und in der Abteilung für Neurologische Frührehabilitation des Klinikums Bremen-Ost.

Vom 01. Mai 2009 bis 30.06.2010 war ich als vollzeitbeschäftigter Assistenzarzt in der Klinik für Geriatrie beschäftigt, wo die Weiterbildung abgeschlossen wurde. Nach Absolvierung der Facharztprüfung erhielte ich am 16.11.2010 die Anerkennung für das Fachgebiet „Physikalische und Rehabilitative Medizin“. Seit dem 01.07.2010 bin ich neben der Vorbereitung für die Facharztprüfung und dem Verfassen meiner Dissertation arbeitssuchend. 